\title{
Iron-Catalyzed Cyanoalkylation of Glycine Derivatives Promoted by Pyridine-Oxazoline Ligands
}

Dengfu Lu* Jiajia Cui ${ }^{\star}$, Sen Yang ${ }^{\star}$ and Yuefa Gong*

School of Chemistry and Chemical Engineering, Huazhong University of Science and Technology

1037 Luoyu Rd., Wuhan, Hubei, 430074, China

E-mails: dlu@hust.edu.cn; gongyf@hust.edu.cn

\section{Table of Contents}

I. General Information

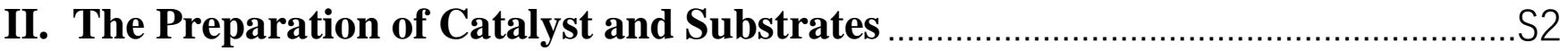

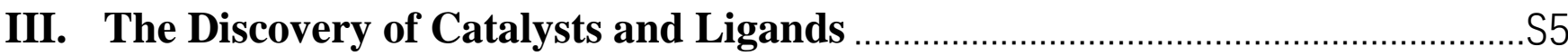

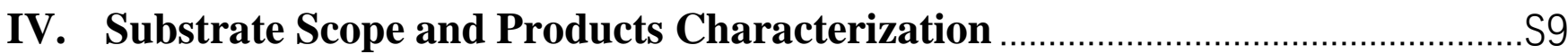

V. Control Experiments and Mechanistic Studies ….............................................. S20

VI. Gram Scale Synthesis \& Product Derivatizations …........................................ S32

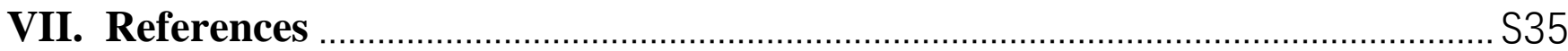

VIII. Copies of NMR Spectra for New Compounds ……......................................... S37 


\section{General Information}

General procedures. All reactions were performed in oven-dried or flame-dried round-bottom flasks and vials. Solvents were dried and freshly distilled before use. Reactions were monitored with thin layer chromatography (TLC) using silica gel 60 F-254 plates. TLC plates were normally visualized by UV irradiation $(254 \mathrm{~nm}$ or $365 \mathrm{~nm})$, stained with basic $\mathrm{KMnO}_{4}$ or phosphomolybdic acid. Flash chromatography was performed using silica gel 60 (200-300 mesh).

Instrumentation. Proton nuclear magnetic resonance $\left({ }^{1} \mathrm{H}\right.$ NMR) spectra and carbon nuclear magnetic resonance $\left({ }^{13} \mathrm{C}\left\{{ }^{1} \mathrm{H}\right\}\right.$ NMR) spectra were recorded on Bruker Ascend $400 \mathrm{MHz}$ and $600 \mathrm{MHz}$. Chemical shifts for protons are reported in parts per million downfield from tetramethylsilane and are referenced to the NMR solvent residual peak $\left(\mathrm{CHCl}_{3}: \delta 7.26\right)$. Chemical shifts for carbons are reported in parts per million downfield from tetramethylsilane and are referenced to the carbon resonances of the NMR solvent (Chloroform- $d: \delta 77.0)$. Data are represented as follows: chemical shift, multiplicity $(\mathrm{s}=$ singlet, $\mathrm{d}=$ doublet, $\mathrm{t}=$ triplet, $\mathrm{q}=$ quartet, $\mathrm{m}=$ multiplet, $\mathrm{br}=$ broad $)$, coupling constants in Hertz $(\mathrm{Hz})$, and integration. IR spectra were recorded on a Bruker FT-IR spectrometer. HRMS was measured on a TOFQ mass spectrometer equipped with an ESI source. High-performance liquid chromatography (HPLC) analysis was recorded on Shimadzu CBM-10A with Daicel chiral columns.

\section{Abbreviations:}

TLC-thin layer chromatography; PE-Petroleum Ethers (60-90); THF-tetrahydrofuran; DMSO-dimethyl sulfoxide; DMF- $N, N$-dimethylformamide; $\quad$ DCE-1,2-dichloroethane; $\quad$ TEMPO-(2,2,6,6tetramethylpiperidin-1-yl)oxyl; CAN-ceric ammonium nitrate; DABCO-1,4-diazabicyclo[2.2.2]octane; LED-light-emitting diode 


\section{The Preparation of Catalyst and Substrates}

a) $\mathbf{F e}\left(\mathbf{N T f _ { 2 }}\right)_{2}$ was prepared from Fe powder and trifluoromethanesulfonimide $\left(\mathrm{HNTf}_{2}\right)$ according to a literature procedure ${ }^{[1-2]}$

b) a-Amino carbonyl compounds

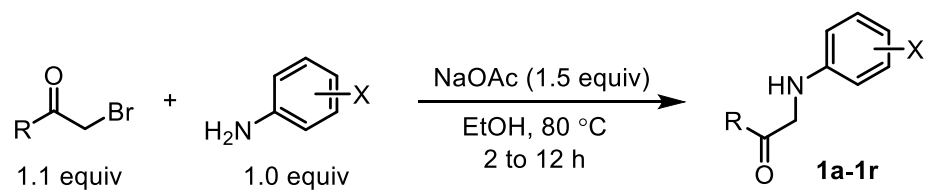<smiles>CCOC(=O)CCNc1ccc(NCC(=O)Nc2ccc(F)cc2)cc1</smiles><smiles>CCOC(=O)CNc1cccc(NCC(=O)OCC)c1</smiles><smiles>COc1ccc(NCC(=O)c2ccccc2)cc1</smiles><smiles>COc1ccc(NCC(=O)Nc2ccccc2)cc1</smiles><smiles>COc1ccc(NCC(=O)Nc2ccccc2)cc1</smiles><smiles>CCOC(=O)CNC(=O)CNc1ccc(OC)cc1</smiles><smiles>COC(=O)C[C@H](Cc1ccccc1)NC(=O)CNc1ccc(OC)cc1</smiles>

The $\alpha$-amino carbonyl compounds were prepared through substitution reactions of corresponding anilines and $\alpha$-bromo carbonyl compounds according to literature procedures. ${ }^{[3-11]}$ Compound 1c, 1d, and $1 \mathrm{~m}$ are new, while the rest of them have been previously reported, and their characterization data are in agreement with the literature.

\begin{tabular}{|c|c|c|c|c|c|c|c|c|}
\hline compounds & $\begin{array}{c}1 \mathbf{a}, \mathbf{1 e}, \mathbf{1 f}, \mathbf{1 g}, \mathbf{1 h}, \mathbf{1 j}, \\
\mathbf{1 k}\end{array}$ & $\mathbf{1 b}$ & $\mathbf{1 i}$ & $\mathbf{1 l , 1 \mathbf { n }}$ & $\mathbf{1 p}$ & $\mathbf{1 0}$ & $\mathbf{1 q}$ & $\mathbf{1 r}$ \\
\hline references & {$[3-4]$} & {$[5]$} & {$[6]$} & {$[7]$} & {$[8]$} & {$[9]$} & {$[10]$} & {$[11]$} \\
\hline
\end{tabular}

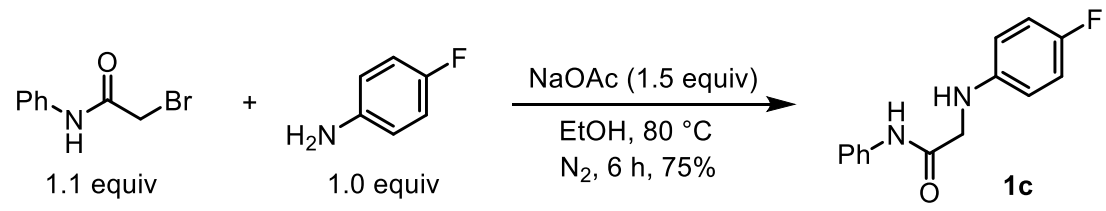

To an oven-dried three-neck flask equipped with a reflux condenser were added 2-bromo- $N$ phenylacetamide (2.35 g, $11 \mathrm{mmol}), \mathrm{NaOAc}(1.23 \mathrm{~g}, 15 \mathrm{mmol}), p$-fluoroaniline $(1.11 \mathrm{~g}, 10 \mathrm{mmol})$, and anhydrous EtOH $(10 \mathrm{~mL})$. The reaction was flushed with $\mathrm{N}_{2}$ and then heated in an $80{ }^{\circ} \mathrm{C}$ oil bath, and the 
progress was monitored with TLC until $p$-fluoroaniline was consumed. Upon completion, the mixture was cooled down to room temperature and quenched with $\mathrm{H}_{2} \mathrm{O}(20 \mathrm{~mL})$. After EtOH was removed in vacuo through rotatory evaporation, the aqueous phase was extracted with $\mathrm{CH}_{2} \mathrm{Cl}_{2}(20 \mathrm{~mL} \times 3)$. The combined organic layers were dried over anhydrous $\mathrm{Na}_{2} \mathrm{SO}_{4}$ and concentrated in vacuo to afford the crude product.

2-((4-Fluorophenyl)amino)-N-phenylacetamide (1c): Pure 1c was obtained by recrystalization from hot hexanes/EtOAc as a light yellow solid $\left(1.83 \mathrm{~g}, 75 \%\right.$ yield, m.p. $\left.115-116{ }^{\circ} \mathrm{C}\right) .{ }^{1} \mathrm{H}$ NMR $(400 \mathrm{MHz}$, Chloroform- $d$ ) $\delta 8.60$ (brs, 1H), $7.58-7.47$ (m, 2H), $7.36-7.28$ (m, 2H), $7.16-7.08$ (m, 1H), $6.99-$ $6.89(\mathrm{~m}, 2 \mathrm{H}), 6.69-6.59(\mathrm{~m}, 2 \mathrm{H}), 4.34(\mathrm{br}, 1 \mathrm{H}), 3.86(\mathrm{~s}, 2 \mathrm{H}) ;{ }^{13} \mathrm{C}\left\{{ }^{1} \mathrm{H}\right\} \mathrm{NMR}\left(101 \mathrm{MHz}, \mathrm{CDCl}_{3}\right) \delta 168.7$, $157.0(\mathrm{~d}, J=237.7 \mathrm{~Hz}), 143.3(\mathrm{~d}, J=2.2 \mathrm{~Hz}), 137.2,129.1,124.7,119.9,116.1(\mathrm{~d}, J=22.6 \mathrm{~Hz}), 114.5$ (d, $J=7.8 \mathrm{~Hz}), 50.3 ;{ }^{19} \mathrm{~F}$ NMR (376 MHz, Chloroform- $d$ ) $\delta-125.08--125.18(\mathrm{~m})$; IR $v_{\max }(\mathrm{neat}) / \mathrm{cm}^{-1}$ : 3324, 3031, 2903, 1669, 1598, 1526, 1426, 1316, 1220, 1111, 819, 760, 695; HRMS (ESI, m/z): calcd for $\mathrm{C}_{14} \mathrm{H}_{13} \mathrm{FN}_{2} \mathrm{ONa}^{+}[\mathrm{M}+\mathrm{Na}]^{+} 267.0904$, found 267.0901 .

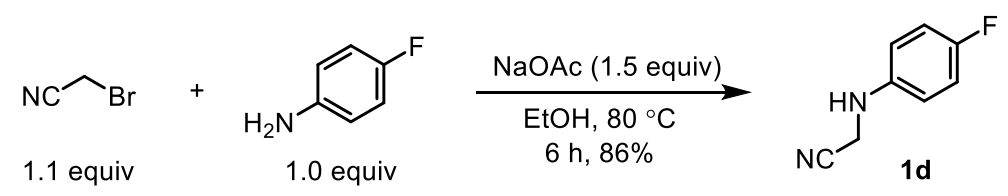

2-((4-Fluorophenyl)amino)acetonitrile (1d): Compound 1d was prepared through a similar procedure with $p$-fluoroaniline and bromoacetonitrile, and its was isolated through a silica gel flash column as a light yellow solid (86\% yield, m.p. $\left.41-42{ }^{\circ} \mathrm{C}\right) .{ }^{1} \mathrm{H}$ NMR (400 MHz, Chloroform- $d$ ) $\delta 7.02-6.94(\mathrm{~m}, 2 \mathrm{H})$, $6.71-6.62(\mathrm{~m}, 2 \mathrm{H}), 4.08(\mathrm{~s}, 2 \mathrm{H}), 3.91(\mathrm{br}, 1 \mathrm{H}) ;{ }^{13} \mathrm{C}\left\{{ }^{1} \mathrm{H}\right\} \mathrm{NMR}(101 \mathrm{~Hz}$, Chloroform- $d) \delta 157.3(\mathrm{~d}, J=$ $237.8 \mathrm{~Hz}), 141.2(\mathrm{~d}, J=2.2 \mathrm{~Hz}), 116.9,116.2(\mathrm{~d}, J=22.5 \mathrm{~Hz}), 114.9(\mathrm{~d}, J=7.6 \mathrm{~Hz}), 33.3 ;{ }^{19} \mathrm{~F}$ NMR $(376$ MHz, Chloroform- $d$ ) $\delta-124.46$ - -124.57 (m); IR $v_{\max }$ (neat)/cm ${ }^{-1}: 3322,3042,2964,2925,2866,2243$, 1662, 1598, 1518, 1444, 1318, 1210, 822, 755, 701; HRMS (ESI, m/z): calcd for $\mathrm{C}_{8} \mathrm{H}_{7} \mathrm{FN}_{2} \mathrm{Na}^{+}[\mathrm{M}+\mathrm{Na}]^{+}$ 173.0485, found 173.0476 .

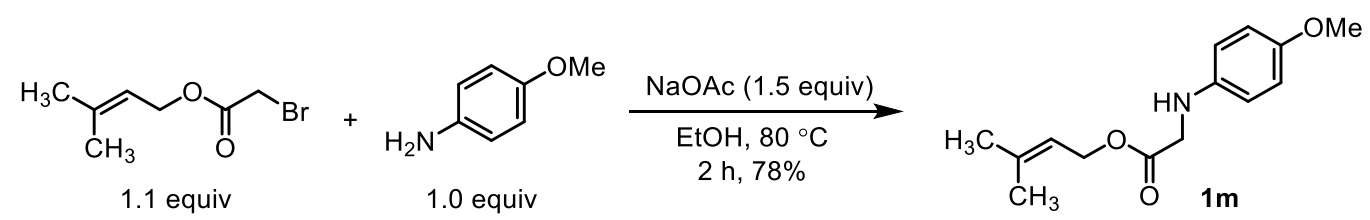

Prenyl (4-methoxyphenyl)glycinate (1m): Compound $1 \mathrm{~m}$ was also synthesized through a similar procedure with prenyl bromoacetate and $p$-methoxyaniline. The crude product was purified through a flash column to give $\mathbf{1 m}$ as a light yellow solid (78\% yield, m.p. $\left.37-38{ }^{\circ} \mathrm{C}\right) .{ }^{1} \mathrm{H}$ NMR $(400 \mathrm{MHz}$, Chloroform- $d$ ) $\delta 6.82-6.76(\mathrm{~m}, 2 \mathrm{H}), 6.62-6.55(\mathrm{~m}, 2 \mathrm{H}), 5.40-5.30(\mathrm{~m}, 1 \mathrm{H}), 4.67(\mathrm{~d}, J=7.3 \mathrm{~Hz}, 2 \mathrm{H})$, $4.00(\mathrm{br}, 1 \mathrm{H}), 3.86(\mathrm{~s}, 2 \mathrm{H}), 3.74(\mathrm{~s}, 3 \mathrm{H}), 1.77(\mathrm{~s}, 3 \mathrm{H}), 1.72(\mathrm{~s}, 3 \mathrm{H}) ;{ }^{13} \mathrm{C}\left\{{ }^{1} \mathrm{H}\right\} \mathrm{NMR}\left(101 \mathrm{MHz}, \mathrm{CDCl}_{3}\right) \delta$ $171.4,152.6,141.3,139.9,118.1,114.9,114.4,62.1,55.7,46.9,25.8,18.0 ;$ IR $v_{\max }($ neat $) / \mathrm{cm}^{-1}: 3360$, 
2961, 2828, 1728, 1515, 1441, 1233, 1199, 1174, 1146, 1033, 819; HRMS (ESI, m/z): calcd for $\mathrm{C}_{14} \mathrm{H}_{20} \mathrm{NO}_{3} \mathrm{Na}^{+}[\mathrm{M}+\mathrm{Na}]^{+}$273.1335, found 273.1336.

c) Cyclobutanone oxime esters
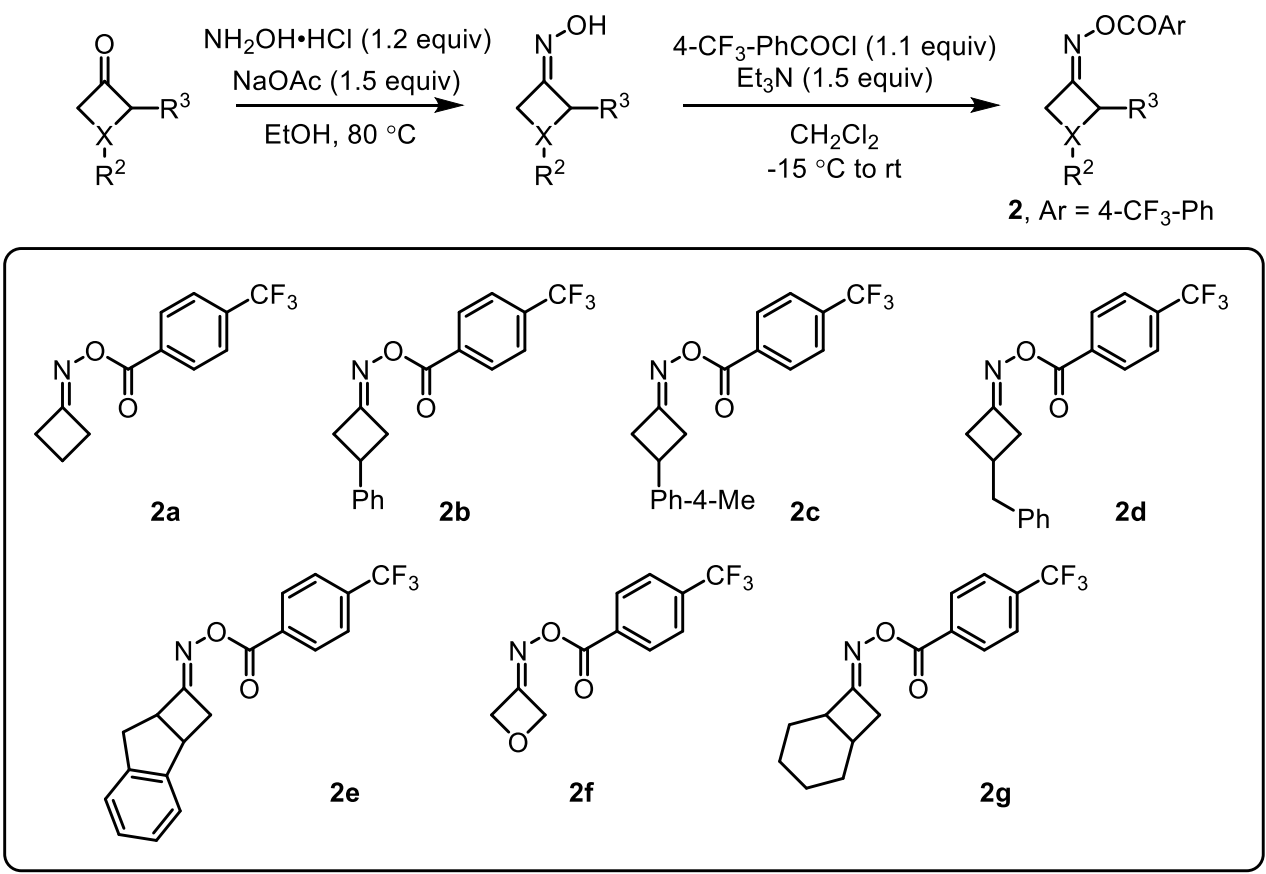

The cyclobutanone oxime esters were prepared according to literature procedures. ${ }^{[12-15]} \mathbf{2 a}$ to $\mathbf{2 f}$ are known compounds and their characterization data are in agreement with the reported ones.

\begin{tabular}{|c|c|c|c|c|}
\hline compound & $\mathbf{2 a}$ & $\mathbf{2 b}, \mathbf{2 f}$ & $\mathbf{2 c}$ & $\mathbf{2 d}, \mathbf{2 e}$ \\
\hline reference & {$[12]$} & {$[13]$} & {$[14]$} & {$[15]$} \\
\hline
\end{tabular}
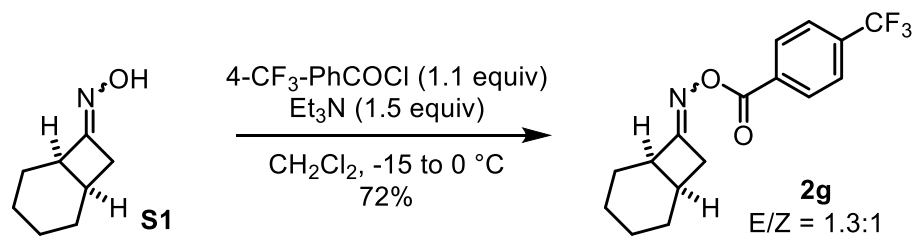

Compound $\mathbf{2 g}$ was prepared through the acylation of oxime $\mathbf{S 1}$, which was obtained according to a literature procedure. ${ }^{[16]}$ Oxime $\mathbf{S 1}(700 \mathrm{mg}, 5 \mathrm{mmol})$ was added to a flame-dried round bottom flash charged with a stir bar and the flask was sealed under $\mathrm{N}_{2}$ with a septum. After dry $\mathrm{CH}_{2} \mathrm{Cl}_{2}(10 \mathrm{~mL})$ and $\mathrm{NEt}_{3}(1 \mathrm{~mL}, 7.5 \mathrm{mmol})$ were added successively, the flask was stirred and cooled in an ice-slat bath. A solution of p-trifluoromethylbenzoyl chloride $\left(1.15 \mathrm{~g}\right.$, $5.5 \mathrm{mmol}$, dissolved in $5 \mathrm{~mL} \mathrm{CH}_{2} \mathrm{Cl}_{2}$ ) was added into the flask slowly. The mixture was stirred at the same temperature for $10 \mathrm{~min}$ and then gradually warmed up to $0{ }^{\circ} \mathrm{C}$ room temperature before it was quenched by $\mathrm{NaHCO}_{3}$ saturated aqueous solution. After the organic layer was separated, the aqueous phase was extracted with $\mathrm{CH}_{2} \mathrm{Cl}_{2}(20 \mathrm{~mL} \times 3)$. The 
combined organic layers were dried over anhydrous $\mathrm{Na}_{2} \mathrm{SO}_{4}$ and concentrated in vacuo to afford the crude product.

Bicyclo[4.2.0]octan-7-one O-(4-(trifluoromethyl)benzoyl) oxime (2g): compound $2 \mathrm{~g}$ was purified through a silica gel flash column to afford a pair of isomers (1.12 g, 72\% yield, white solid, m.p. 47-49 $\left.{ }^{\circ} \mathrm{C}\right)$. IR $v_{\max }($ neat $) / \mathrm{cm}^{-1}: 2932,2849,1745,1681,1410,1328,1258,1163,1059,854,770 ;{ }^{1} \mathrm{H}$ NMR $(400$ MHz, Chloroform- $d$ ) $\delta 8.20-8.07(\mathrm{~m}, 2 \mathrm{H}), 7.64-7.77(\mathrm{~m}, 2 \mathrm{H}), 3.50-3.35(\mathrm{~m}, 1 \mathrm{H}), 2.97-3.01(\mathrm{~m}, 1 \mathrm{H})$, $2.86-2.66(\mathrm{~m}, 1 \mathrm{H}), 2.54-2.40(\mathrm{~m}, 1 \mathrm{H}), 2.05-1.76(\mathrm{~m}, 2 \mathrm{H}), 1.75-1.18(\mathrm{~m}, 6 \mathrm{H}) ;{ }^{13} \mathrm{C}\left\{{ }^{1} \mathrm{H}\right\} \mathrm{NMR}(101$ MHz, Chloroform- $d$ ) $\delta$ 171.6, 171.3, 163.1, 163.0, 134.6 (q, $J=32.8 \mathrm{~Hz}), 134.5$ (q, $J=32.8 \mathrm{~Hz}), 132.6$, $132.5,130.0,129.90,125.6(\mathrm{q}, J=3.8 \mathrm{~Hz}), 125.5(\mathrm{q}, J=3.8 \mathrm{~Hz}), 123.56(\mathrm{q}, J=272.8 \mathrm{~Hz}), 123.54(\mathrm{q}, J$ $=272.8 \mathrm{~Hz}), 44.4$, 43.2, 37.1, 35.9, 27.8, 26.7, 26.7, 25.9, 23.8, 23.3, 21.6, 21.5, 21.4, 21.0; ${ }^{19} \mathrm{~F} \mathrm{NMR}$ (376 MHz, Chloroform- $d$ ) $\delta-63.17$ (s, 3F, major), -63.19 (s, 3F, minor); HRMS (ESI, m/z): calcd for $\mathrm{C}_{16} \mathrm{H}_{17} \mathrm{~F}_{3} \mathrm{NO}_{2}^{+}[\mathrm{M}+\mathrm{H}]^{+}$312.1206, found 312.1203.

\section{The Discovery of Catalysts and Ligands}

\section{a) Selectivity studies with different metal salts}

Procedures for $\mathrm{Cu}, \mathrm{Fe}$, and Co-catalyzed reactions: To a flame-dried reaction tube charged with a stir bar were added glycinate 1a $(39.4 \mathrm{mg}, 0.2 \mathrm{mmol})$, cyclobutanone oxime ester $\mathbf{2 a}$ (57 mg, $0.22 \mathrm{mmol})$, and a corresponding metal salt $(0.01 \mathrm{mmol})$. The tube was sealed with a septum, evacuated, and backfilled with $\mathrm{N}_{2}$ three times. After anhydrous 1,2-dichloroethane $(2 \mathrm{~mL})$ were added, the reaction was stirred at an indicated temperature in an oil bath, and the reaction progress was monitored by TLC. After the indicated time, the reaction was quenched with saturated $\mathrm{NaHCO}_{3}$ aqueous solution $(2 \mathrm{~mL})$ and the aqueous phase was extracted with $\mathrm{CH}_{2} \mathrm{Cl}_{2}(1 \mathrm{~mL} \times 3)$. The combined organic layers were concentrated in vacuo, filtered through a short silica gel pad, and washed with PE/EtOAc (4:1, $15 \mathrm{~mL})$. After the eluent was removed in vacuo, the residue was dissolved in $\mathrm{CDCl}_{3}$ together with 1,3,5-trimethoxyl benzene and submitted for ${ }^{1} \mathrm{H}$ and ${ }^{19} \mathrm{~F}$ NMR analysis.

Procedures for $f a c$-Ir(ppy)3-catalyzed reactions: To a flame-dried sealable 2-dram vial charged with a stir bar were added glycinate $\mathbf{1 a}(39.4 \mathrm{mg}, 0.2 \mathrm{mmol})$, cyclobutyl oxime ester $\mathbf{2 a}(57 \mathrm{mg}, 0.22 \mathrm{mmol})$, $\operatorname{Ir}($ ppy) $3(0.7 \mathrm{mg}, 0.002 \mathrm{~mol})$ and DABCO (if added, $26.9 \mathrm{mg}, 0.24 \mathrm{mmol})$. After the vial was evacuated and backfilled with $\mathrm{N}_{2}$ three times, anhydrous 1,2-dichloroethane ( $2 \mathrm{~mL}$ ) was added. The resulting yellow solution was stirred and irradiated with blue light (6 W LEDs) at room temperature $\left(23\right.$ to $\left.26^{\circ} \mathrm{C}\right)$ for $5 \mathrm{~h}$. The reaction was quenched with saturated $\mathrm{NaHCO}_{3}$ aqueous solution $(2 \mathrm{~mL})$. After the organic layer was separated, the aqueous phase was extracted with $\mathrm{CH}_{2} \mathrm{Cl}_{2}(1 \mathrm{~mL} \times 3)$. The combined organic layers were concentrated in vacuo, filtered through a short silica gel pad, and washed with PE/EtOAc (4:1, $15 \mathrm{~mL})$. 
After the eluent was removed in vacuo, the residue was dissolved in $\mathrm{CDCl}_{3}$ together with 1,3,5trimethoxyl benzene and submitted for ${ }^{1} \mathrm{H}$ and ${ }^{19} \mathrm{~F}$ NMR analysis.

The reaction time, conversions of 1a, and yields of products obtained under different conditions are summarized in Table S1.

Table S1. The Discovery of Catalysts and Ligands ${ }^{a}$

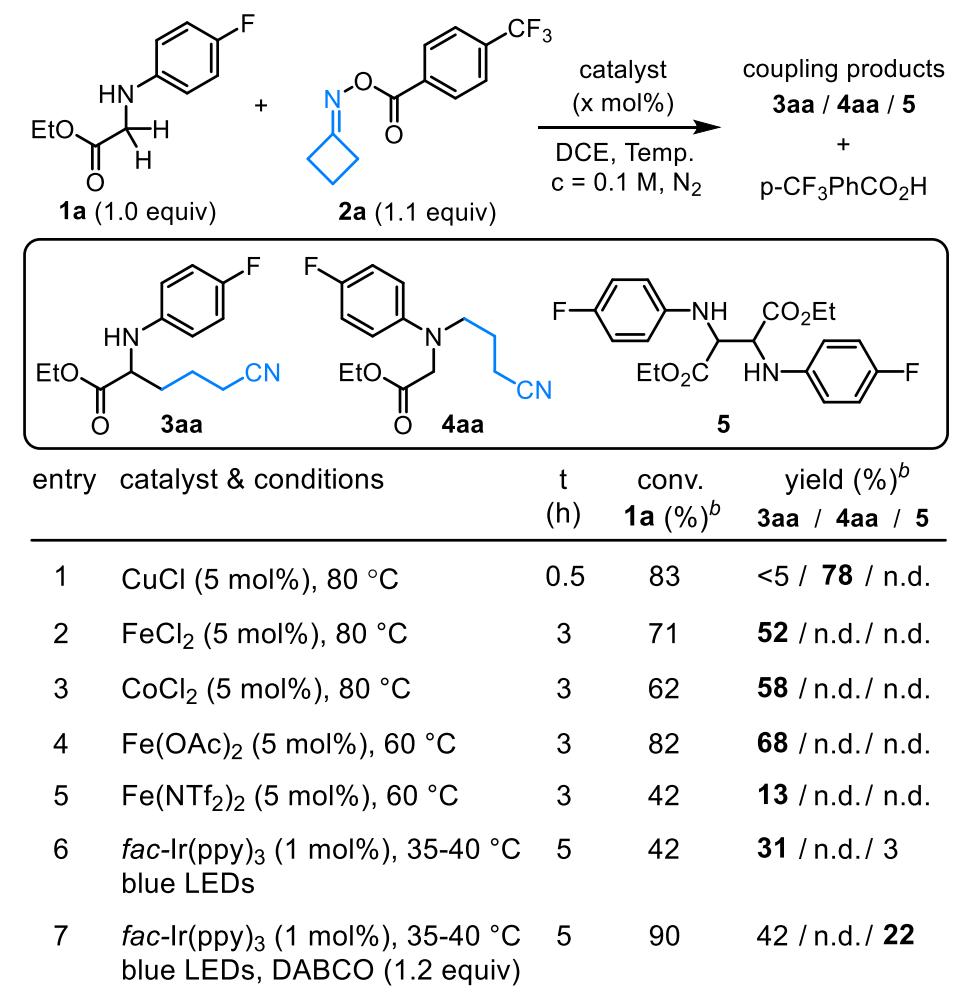

${ }^{a}$ Unless otherwise stated, the reaction was performed under $\mathrm{N}_{2}$ with glycine ester $1 \mathbf{1 a}(0.2 \mathrm{mmol})$, oxime ester $2 \mathbf{a}(0.22 \mathrm{mmol})$ in the presence of an indicated catalyst in 1,2-dichloroethane $(2 \mathrm{~mL}) .{ }^{b}$ Conversions and yields based on $1 \mathrm{a}$ were determined by NMR analysis with an internal standard. n.d. $=$ not detected by ${ }^{1} \mathrm{H}$ or ${ }^{19} \mathrm{~F} \mathrm{NMR}$.

\section{b) The evaluation of ligands for iron catalyst}

Procedures for ligand evaluation: In a $\mathrm{N}_{2}$ atmosphere glovebox, $\mathrm{Fe}\left(\mathrm{NTf}_{2}\right)_{2}(6.2 \mathrm{mg}, 0.01 \mathrm{mmol})$, and a ligand $(0.01$ or $0.02 \mathrm{mmol})$ were added to a 1-dram vial, which was then charged with a stir bar and sealed under $\mathrm{N}_{2}$ with a septum. After the vial was then taken out of the glove box, freshly distilled 1,2dichloroethane $(0.9 \mathrm{~mL})$ and acetonitrile $(0.1 \mathrm{~mL})$ was added to afford a clear catalyst solution. To a flame-dried reaction tube charged with a stir bar were added glycinate 1a (39.4 $\mathrm{mg}, 0.2 \mathrm{mmol})$ and cyclobutanone oxime ester $\mathbf{2 a}(57 \mathrm{mg}, 0.22 \mathrm{mmol})$. The tube was sealed with a septum, evacuated, and backfilled with $\mathrm{N}_{2}$ three times. Anhydrous 1,2-dichloroethane $(1.5 \mathrm{~mL})$ was added, followed by the addition of the above catalyst solution $(0.5 \mathrm{~mL}, 2.5 \mathrm{~mol} \%$ based on $\mathrm{Fe})$. The reaction was stirred at room temperature ( 23 to $26{ }^{\circ} \mathrm{C}$ ) and the progress was monitored by TLC. With L2 as the ligand, a white precipitate (4-trifluoromethyl benzoic acid) quickly formed as the oxime ester decomposed. After the indicated time, the reaction was quenched with saturated $\mathrm{NaHCO}_{3}$ aqueous solution $(2 \mathrm{~mL})$ and extracted 
with $\mathrm{CH}_{2} \mathrm{Cl}_{2}(1 \mathrm{~mL} \times 3)$. The combined organic layers were concentrated in vacuo, filtered through a short silica gel pad, and washed with PE/EtOAc (4:1, $15 \mathrm{~mL})$. After the eluent was removed in vacuo, the residue was dissolved in $\mathrm{CDCl}_{3}$ together with 1,3,5-trimethoxyl benzene and submitted for ${ }^{1} \mathrm{H}$ and ${ }^{19} \mathrm{~F}$ NMR analysis.

The reaction time, conversions of 1a, and yields of 3aa obtained under different conditions are summarized in Table S2.

Table S2. The Evaluation of Ligands for Iron Catalyst ${ }^{a}$

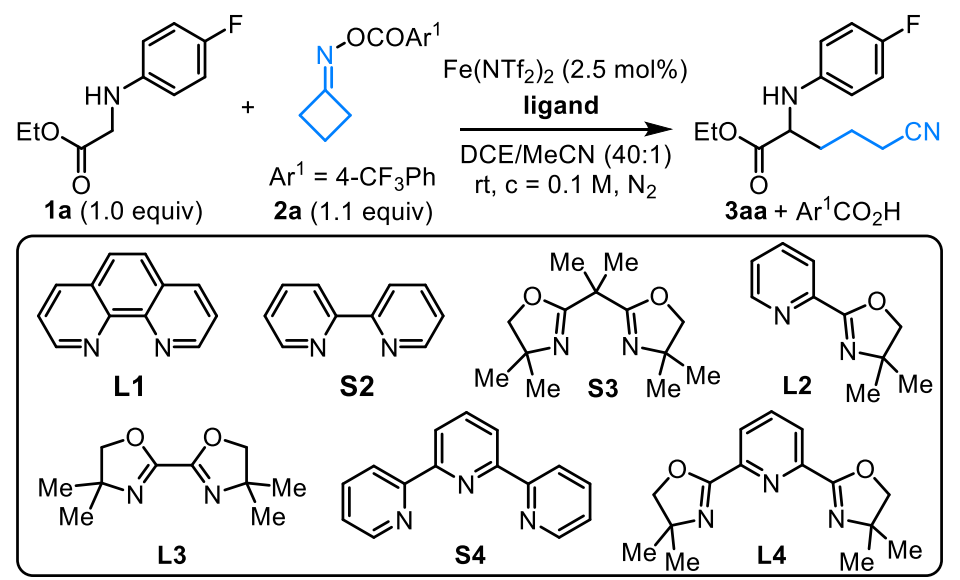

\begin{tabular}{|c|c|c|c|c|c|}
\hline \multirow[t]{2}{*}{ entry } & \multirow{2}{*}{$\begin{array}{l}\text { Ligand (mol\%) } \\
\text { and other variations }\end{array}$} & \multirow{2}{*}{$\begin{array}{c}\mathrm{t} \\
(\mathrm{h})\end{array}$} & \multirow{2}{*}{$\begin{array}{c}\text { conversion } \\
\text { of } 1 \mathrm{a}(\%)^{b}\end{array}$} & \multicolumn{2}{|c|}{ yield $(\%)^{b}$} \\
\hline & & & & 3aa & / 4aa / 5 \\
\hline 1 & no ligand & 5 & $<5$ & n.d. & / n.d. / n.d. \\
\hline 2 & L1 (5) & 5 & $<5$ & n.d. & / n.d. / n.d. \\
\hline 3 & $\mathbf{S 2}(5)$ & 5 & $<5$ & n.d. & / n.d. / n.d. \\
\hline 4 & S3 (5) & 5 & 32 & 26 & / n.d. / n.d. \\
\hline 5 & L2 (5) & 1 & 92 & 84 & / n.d. / n.d. \\
\hline 6 & L3 (5) & 5 & $<5$ & n.d. & / n.d. / n.d. \\
\hline 7 & S4 (2.5) & 5 & $<5$ & n.d. & / n.d. / n.d. \\
\hline 8 & L4 (2.5) & 2 & 75 & 68 & / n.d. / n.d. \\
\hline 9 & $\mathrm{FeCl}_{2}(2.5)+\mathrm{L2}(5)$ & 5 & $<5$ & n.d. & / n.d. / n.d. \\
\hline 10 & $\mathrm{Fe}(\mathrm{OAc})_{2}(2.5)+\mathrm{L2}(5)$ & 5 & $<5$ & n.d. & / n.d. / n.d. \\
\hline 11 & L2 (2.5) & 0.5 & 90 & 78 & / n.d. / n.d. \\
\hline 12 & L2 (5), $\mathbf{2 a}$ (1.2 equiv) & 1 & $>95$ & 89 & / n.d. / n.d. \\
\hline $13^{c}$ & $\begin{array}{l}\mathrm{Fe}\left(\mathrm{NTf}_{2}\right)_{2}(1), \mathrm{L} 2(2) \\
\mathbf{2 a}(1.2 \text { equiv.) }\end{array}$ & 5 & $>95$ & $91^{d}$ & / n.d. / n.d. \\
\hline
\end{tabular}

${ }^{a}$ Unless otherwise stated, the reaction was performed under $\mathrm{N}_{2}$ with glycine ester $1 \mathbf{a}(0.2 \mathrm{mmol})$, oxime ester $2 \mathbf{a}(0.22 \mathrm{mmol})$ in the presence of $\mathrm{Fe}\left(\mathrm{NTf}_{2}\right)_{2}$ and a ligand in 1,2-dichloroethane $(2 \mathrm{~mL})$ at room temperature. ${ }^{b}$ Conversions and yields based on $\mathbf{1 a}$ were determined by NMR analysis with an internal standard. ${ }^{c}$ Reaction on $0.5 \mathrm{mmol}$ scale. ${ }^{d}$ Isolated yield. n.d. $=$ not detected by ${ }^{1} \mathrm{H}$ or ${ }^{19} \mathrm{~F}$ NMR.<smiles>CCOC(=O)C(CCCC#N)Nc1ccc(F)cc1</smiles>

Ethyl 5-cyano-2-((4-fluorophenyl)amino)pentanoate (3aa): compound 3aa was isolated from the 0.5 mmol scale reaction (Table S2, entry 13) through a silica gel flash column (PE: EtOAc from 10:1 to 5:1) 
as a light yellow solid (120 mg, 91\% yield, mp: $\left.46-47{ }^{\circ} \mathrm{C}\right) .{ }^{1} \mathrm{H}$ NMR (400 MHz, Chloroform- $d$ ) $\delta 6.95$ - $6.82(\mathrm{~m}, 2 \mathrm{H}), 6.61-6.51(\mathrm{~m}, 2 \mathrm{H}), 4.18$ (q, $J=7.1 \mathrm{~Hz}, 2 \mathrm{H}), 4.06$ (br, 1H), 3.99 (t, $J=6.3 \mathrm{~Hz}, 1 \mathrm{H}), 2.48$ $-2.33(\mathrm{~m}, 2 \mathrm{H}), 2.05-1.93(\mathrm{~m}, 1 \mathrm{H}), 1.95-1.75(\mathrm{~m}, 3 \mathrm{H}), 1.24(\mathrm{t}, J=7.1 \mathrm{~Hz}, 3 \mathrm{H}) ;{ }^{13} \mathrm{C}\left\{{ }^{1} \mathrm{H}\right\} \mathrm{NMR}(101$ MHz, Chloroform- $d$ ) $\delta 173.3,156.5(\mathrm{~d}, J=236.5 \mathrm{~Hz}), 142.9(\mathrm{~d}, J=2.1 \mathrm{~Hz}), 119.1,115.9(\mathrm{~d}, J=22.5$ $\mathrm{Hz}), 114.8(\mathrm{~d}, J=7.5 \mathrm{~Hz}), 61.5,56.8,31.7,21.8,17.0,14.2 ;{ }^{19} \mathrm{~F}$ NMR $(376 \mathrm{MHz}$, Chloroform- $d$ ) $\delta-125.2$ - -127.0 (m); IR $v_{\max }\left(\right.$ neat) $/ \mathrm{cm}^{-1}: 3365,2932,2246,1728,1613,1508,1497,1210,1019$; HRMS (ESI, $\mathrm{m} / \mathrm{z}$ ): calcd for $\mathrm{C}_{14} \mathrm{H}_{17} \mathrm{FN}_{2} \mathrm{O}_{2} \mathrm{Na}^{+}[\mathrm{M}+\mathrm{Na}]^{+} 287.1166$, found 287.1163.

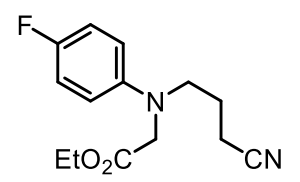

Ethyl N-(3-cyanopropyl)-N-(4-fluorophenyl)glycinate (4aa): compound 4aa was isolated from the $\mathrm{CuCl}$ catalyzed reaction through a silica gel flash column (PE: EtOAc from 10:1 to 5:1) as yellowish oil. ${ }^{1} \mathrm{H}$ NMR (400 MHz, Chloroform- $d$ ) $\delta 7.00-6.88(\mathrm{~m}, 2 \mathrm{H}), 6.68-6.57(\mathrm{~m}, 2 \mathrm{H}), 4.19$ (q, $J=7.1 \mathrm{~Hz}, 2 \mathrm{H})$, $4.00(\mathrm{~s}, 2 \mathrm{H}), 3.51(\mathrm{t}, J=6.8 \mathrm{~Hz}, 2 \mathrm{H}), 2.46(\mathrm{t}, J=6.9 \mathrm{~Hz}, 2 \mathrm{H}), 1.97(\mathrm{p}, J=6.9 \mathrm{~Hz}, 2 \mathrm{H}), 1.26(\mathrm{t}, J=7.2$ $\mathrm{Hz}, 3 \mathrm{H}) ;{ }^{13} \mathrm{C}\left\{{ }^{1} \mathrm{H}\right\} \mathrm{NMR}(101 \mathrm{MHz}$, Chloroform- $d) \delta 170.9,156.2(\mathrm{~d}, J=237.2 \mathrm{~Hz}), 144.1(\mathrm{~d}, J=2.0 \mathrm{~Hz})$, 119.4, $115.8(\mathrm{~d}, J=22.2 \mathrm{~Hz}), 114.5(\mathrm{~d}, J=7.5 \mathrm{~Hz}), 61.1,54.7,50.8,23.7,14.6,14.2 ;{ }^{19} \mathrm{~F} \mathrm{NMR}(376 \mathrm{MHz}$, Chloroform- $d$ ) $\delta-127.09$ - -126.97 (m); IR $v_{\max }$ (neat)/cm ${ }^{-1}: 2929,2245,1739,1509,1229,1192,1162$, 1028; HRMS (ESI, m/z): calcd for $\mathrm{C}_{14} \mathrm{H}_{18} \mathrm{FN}_{2} \mathrm{O}_{2}{ }^{+}[\mathrm{M}+\mathrm{H}]^{+} 265.1347$, found 265.1345.

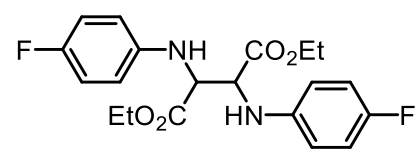

Diethyl 2,3-bis((4-fluorophenyl)amino)succinate (5): compound 5 was isolated from the photoredox catalyzed reaction (with DABCO) through a silica gel flash column (PE: EtOAc from 20:1 to 1:1) as a mixture of diastereomers $(d r=1.4: 1) .{ }^{1} \mathrm{H}$ NMR (400 MHz, Chloroform- $d$ ) $\delta 6.95-6.84(\mathrm{~m}, 4 \mathrm{H}$, major + minor), $6.68-6.59(\mathrm{~m}, 4 \mathrm{H}$, major + minor), $4.57(\mathrm{~d}, J=8.9 \mathrm{~Hz}, 2 \mathrm{H}$, minor), $4.50(\mathrm{~d}, J=9.1 \mathrm{~Hz}, 2 \mathrm{H}$, major), $4.43(\mathrm{~d}, J=9.1 \mathrm{~Hz}, 2 \mathrm{H}$, major), 4.40 (d, $J=8.9 \mathrm{~Hz}, 2 \mathrm{H}$, minor), $4.30-4.17$ (m, 4H, major), 4.19 (q, $J=7.3 \mathrm{~Hz}, 4 \mathrm{H}$, minor), 1.29 (t, $J=7.1 \mathrm{~Hz}, 6 \mathrm{H}$, major), $1.24\left(\mathrm{t}, J=7.2 \mathrm{~Hz}, 6 \mathrm{H}\right.$, minor); ${ }^{13} \mathrm{C}\left\{{ }^{1} \mathrm{H}\right\} \mathrm{NMR}$ (101 MHz, Chloroform- $d$ ) $\delta 171.1$ (minor), 170.6 (major), 156.9 (d, J = 237.1 Hz, minor), 156.8 (d, $J=$ $237.4 \mathrm{~Hz}$, major), 142.9 (d, $J=2.0 \mathrm{~Hz}$, minor), 142.7 (d, $J=1.9 \mathrm{~Hz}$, major), 115.9 (d, $J=22.5 \mathrm{~Hz}$, major), 115.82 (d, $J=7.6 \mathrm{~Hz}$, minor), 115.81 (d, $J=22.4 \mathrm{~Hz}$, minor), 115.5 (d, $J=7.7 \mathrm{~Hz}$, major); 62.2 (minor), 61.9 (major), 60.4 (minor), 59.9 (major), 14.18 (minor), 14.16 (major); ${ }^{19}$ F NMR (376 MHz, Chloroformd) $\delta$-125.29 - -125.41 (m, minor), -125.50 - -125.61 (m, major); IR $v_{\max }$ (neat)/cm ${ }^{-1}: 3347,2957,2925$, 2853, 1742, 1717, 1535, 1509, 1369, 1307, 1220, 1188, 1021, 824; HRMS (ESI, m/z): calcd for $\mathrm{C}_{20} \mathrm{H}_{23} \mathrm{~F}_{2} \mathrm{~N}_{2} \mathrm{O}_{4}{ }^{+}[\mathrm{M}+\mathrm{H}]^{+}$393.1620, found 393.1616. 


\section{c) The catalyst homogeneity studies}

i) HRMS analysis

In order to confirm whether the iron salt forms homogeneous species with $\mathbf{L 2}$, the $\mathrm{Fe}\left(\mathrm{NTf}_{2}\right)_{2} / \mathbf{L 2}(1: 2)$ solution was first submitted for HRMS analysis. The two major ion peaks were assigned to $\mathrm{Fe}(\mathbf{L} 2)\left(\mathrm{NTf}_{2}\right)^{+}$ and $\mathrm{Fe}(\mathbf{L 2})_{2}\left(\mathrm{NTf}_{2}\right)^{+}$, respectively (Figure S1).

$\mathrm{Fe}(\mathbf{L 2})\left(\mathrm{NTf}_{2}\right)^{+}$: HRMS (ESI, m/z): calcd for $\mathrm{C}_{12} \mathrm{H}_{12} \mathrm{~F}_{6} \mathrm{FeN}_{3} \mathrm{O}_{5} \mathrm{~S}_{2}{ }^{+} 511.9466$, found 511.9485 .

$\mathrm{Fe}(\mathbf{L 2})_{2}\left(\mathrm{NTf}_{2}\right)^{+}$: HRMS (ESI, m/z): calcd for $\mathrm{C}_{22} \mathrm{H}_{24} \mathrm{~F}_{6} \mathrm{FeN}_{5} \mathrm{O}_{6} \mathrm{~S}_{2}{ }^{+}$688.0416, found 688.0437 .

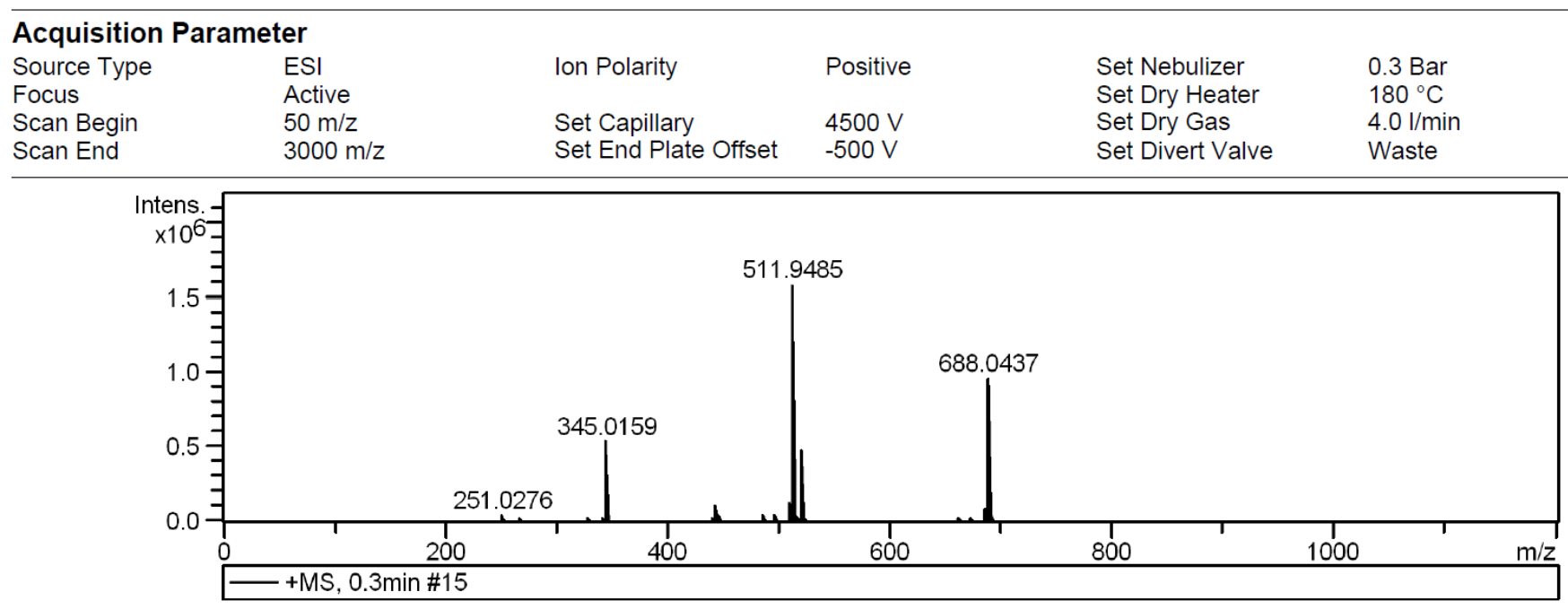

Figure S1. The HRMS result for Fe(NTf $)_{2} / \mathrm{L} 2(1: 2)$ solution

ii) The mercury drop test

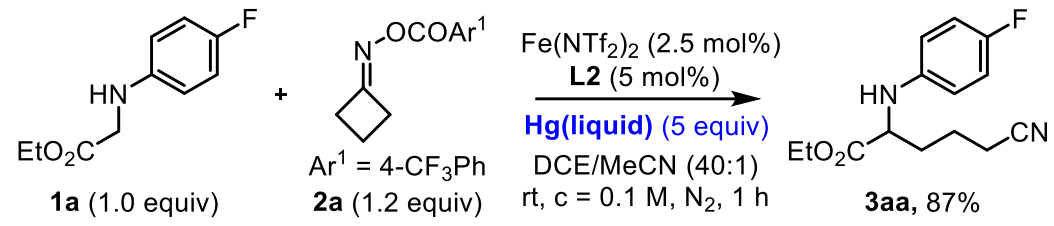

Then, a mercury drop test was performed to determine whether the reaction was promoted by in situformed iron nanoparticles. The reaction was carried out with the same same procedure (Table S2, entry $5)$, but in the presence of 5 equivalent of mercury (200:1 based on iron). The reaction occured rapidly to afford the desired product 3aa in $87 \%$ yield in 1 hour, suggesting the catalytic reaction to be homogeneous.

\section{Substrate Scope and Products Characterization}

The substrate exploration was performed under either condition A or condition B through corresponding procedures, and the obtained nitrile-containing amino acids and peptides derivatives are fully characterized with ${ }^{1} \mathrm{H},{ }^{13} \mathrm{C},{ }^{19} \mathrm{~F}$ NMR, FT-IR, and HRMS. 
a) Condition $\mathbf{A}$ : $\mathrm{Fe}\left(\mathrm{NTf}_{2}\right)_{2} / \mathbf{L 2}(1: 2)$ as the catalyst

General procedures: In a $\mathrm{N}_{2}$ atmosphere glovebox, $\mathrm{Fe}\left(\mathrm{NTf}_{2}\right)_{2}(24.6 \mathrm{mg}, 0.04 \mathrm{mmol})$, and $\mathbf{L 2}(14.1 \mathrm{mg}$, $0.08 \mathrm{mmol}$ ) were weighed into a 2-dram vial charged with a stir bar. The vial was sealed under $\mathrm{N}_{2}$ with a septum and taken out of the glovebox. Then, freshly distilled dry 1,2-dichloroethane $(3.6 \mathrm{~mL})$ and dry MeCN $(0.4 \mathrm{~mL})$ was added into the vial. The mixture was stirred at room temperature to afford a brown catalyst solution ( $\mathrm{c}=0.01 \mathrm{M}, 0.2 \mathrm{ml}$ for each $0.2 \mathrm{mmol}$ scale reaction). Outside the glovebox, to another flame-dried sealable 2-dram vial charged with a stir bar were added a corresponding glycinate $1(0.2$ mmol) and cyclobutanone oxime ester $2 \mathbf{a}(62 \mathrm{mg}, 0.24 \mathrm{mmol})$. After the vial was evacuated and backfilled with $\mathrm{N}_{2}$ three times, anhydrous 1,2-dichloroethane $(1.8 \mathrm{~mL})$ was added, followed by the addition of the catalyst solution $(0.2 \mathrm{~mL})$. The resulting mixture was stirred at room temperature $\left(23\right.$ to $\left.26{ }^{\circ} \mathrm{C}\right)$ and the reaction progress was monitored by TLC. Normally, a white precipitate (4-trifluoromethyl benzoic acid) quickly formed as the oxime ester decomposed. After the indicated time, the reaction was quenched with saturated $\mathrm{NaHCO}_{3}$ aqueous solution $(2 \mathrm{~mL})$ and the aqueous phase was extracted with $\mathrm{CH}_{2} \mathrm{Cl}_{2}(1 \mathrm{~mL} \times$ 3). The combined organic layers were dried over anhydrous $\mathrm{Na}_{2} \mathrm{SO}_{4}$, filtered, and then concentrated in vacuo to afford the crude product, which was purified through a silica gel flash column with petroleum ethers $\left(\mathrm{PE}, 60-90^{\circ} \mathrm{C}\right)$ and EtOAc as eluent.
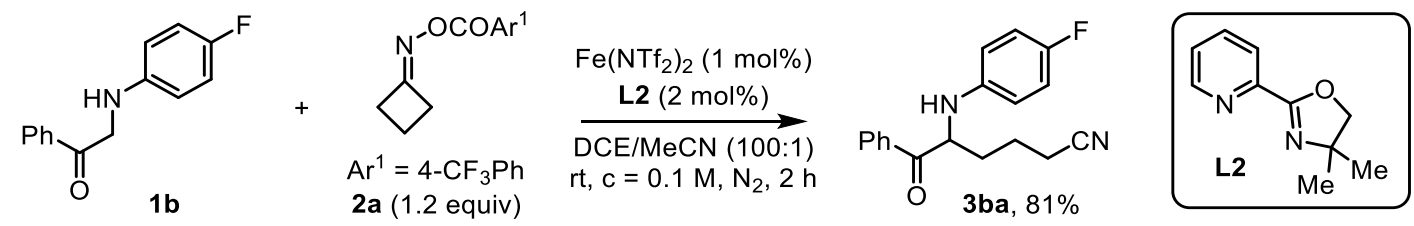

5-((4-Fluorophenyl)amino)-6-oxo-6-phenylhexanenitrile (3ba): Under condition A, compound 3ba was prepared by reacting $\mathbf{1 b}$ with $\mathbf{2 a}$, and the product was isolated through a silica gel flash column (PE: EtOAc, from 5:1 to 2:1) as yellow oil (48 mg, 81\% yield). ${ }^{1} \mathrm{H}$ NMR (400 MHz, Chloroform- $d$ ) $\delta 8.04-$ $7.93(\mathrm{~m}, 2 \mathrm{H}), 7.67-7.59(\mathrm{~m}, 1 \mathrm{H}), 7.57-7.47(\mathrm{~m}, 2 \mathrm{H}), 6.96-6.82(\mathrm{~m}, 2 \mathrm{H}), 6.70-6.57(\mathrm{~m}, 2 \mathrm{H}), 5.03(\mathrm{t}$, $J=5.1 \mathrm{~Hz}, 1 \mathrm{H}), 4.56(\mathrm{br}, 1 \mathrm{H}), 2.35(\mathrm{t}, J=6.5 \mathrm{~Hz}, 2 \mathrm{H}), 2.22-2.07(\mathrm{~m}, 1 \mathrm{H}), 1.90-1.67(\mathrm{~m}, 3 \mathrm{H})$; ${ }^{13} \mathrm{C}\left\{{ }^{1} \mathrm{H}\right\}$ NMR (101 MHz, Chloroform- $d$ ) $\delta 199.8,156.4(\mathrm{~d}, J=236.3 \mathrm{~Hz}), 143.1(\mathrm{~d}, J=2.1 \mathrm{~Hz}), 134.6$, 134.1, 129.1, 128.3, 119.1, $116.0(\mathrm{~d}, J=22.5 \mathrm{~Hz}), 114.9$ (d, $J=7.6 \mathrm{~Hz}), 58.1,31.9,21.3,17.1$; ${ }^{19} \mathrm{~F}$ NMR (376 MHz, Chloroform- $d$ ) $\delta-126.53--125.38$ (m); IR $v_{\max }$ (neat) $/ \mathrm{cm}^{-1}: 3365,2931,2245,1681,1507$, 1447, 1213, 820; HRMS (ESI, m/z): calcd for $\mathrm{C}_{18} \mathrm{H}_{18} \mathrm{FN}_{2} \mathrm{O}^{+}[\mathrm{M}+\mathrm{H}]^{+} 297.1398$, found 297.1382.
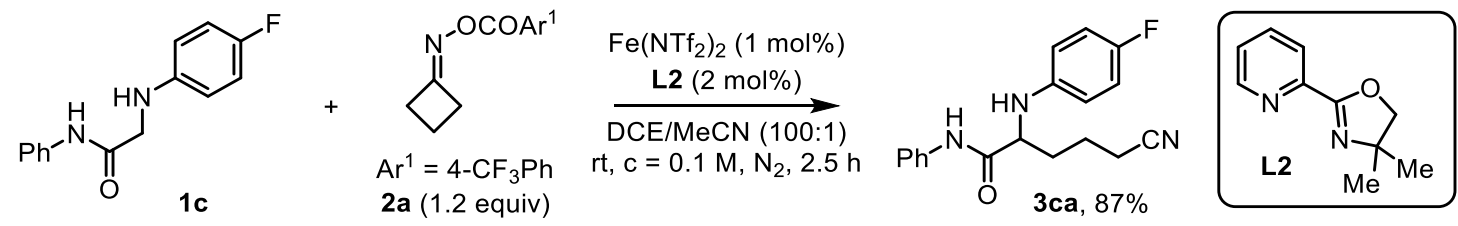
5-cyano-2-((4-fluorophenyl)amino)-N-phenylpentanamide (3ca): Following the general procedure A, compound 3ca was prepared by reacting 1c with $\mathbf{2 a}$, and the product was isolated through a silica gel flash column (PE: EtOAc, from 5:1 to 2:1) as light yellow solid (54 mg, 87\% yield, m.p. $162-164{ }^{\circ} \mathrm{C}$ ). ${ }^{1} \mathrm{H}$ NMR (400 MHz, Chloroform- $d$ ) $\delta 8.65$ (brs, 1H), 7.53 - 7.47 (m, 2H), 7.35 - 7.28 (m, 2H), 7.15 $7.09(\mathrm{~m}, 1 \mathrm{H}), 6.96-6.90(\mathrm{~m}, 2 \mathrm{H}), 6.66-6.59(\mathrm{~m}, 2 \mathrm{H}), 3.91(\mathrm{br}, 1 \mathrm{H}), 3.74(\mathrm{t}, J=6.6 \mathrm{~Hz}, 1 \mathrm{H}), 2.55-$ $2.36(\mathrm{~m}, 2 \mathrm{H}), 2.22-2.10(\mathrm{~m}, 1 \mathrm{H}), 2.06-1.97(\mathrm{~m}, 1 \mathrm{H}), 1.96-1.86(\mathrm{~m}, 2 \mathrm{H}) ;{ }^{13} \mathrm{C}\left\{{ }^{1} \mathrm{H}\right\} \mathrm{NMR}(101 \mathrm{MHz}$, Chloroform- $d$ ) $\delta 170.7,157.1(\mathrm{~d}, J=238.4 \mathrm{~Hz}), 142.4(\mathrm{~d}, J=2.2 \mathrm{~Hz}), 137.1,129.1,124.8,119.9,119.1$, $116.21(\mathrm{~d}, J=22.6 \mathrm{~Hz}), 115.00(\mathrm{~d}, J=7.7 \mathrm{~Hz}), 60.4,32.6,22.3,17.1$; ${ }^{19} \mathrm{~F}$ NMR (376 MHz, Chloroformd) $\delta-124.47$ - -124.65 (m); IR $v_{\max }\left(\right.$ neat) $/ \mathrm{cm}^{-1}: 3322,3042,2925,2243,1662,1598,1518,1442,1318$, 1210, 822, 755, 701; HRMS (ESI, m/z): calcd for $\mathrm{C}_{18} \mathrm{H}_{19} \mathrm{FN}_{3} \mathrm{O}^{+}[\mathrm{M}+\mathrm{H}]^{+} 312.1507$, found 312.1498 .
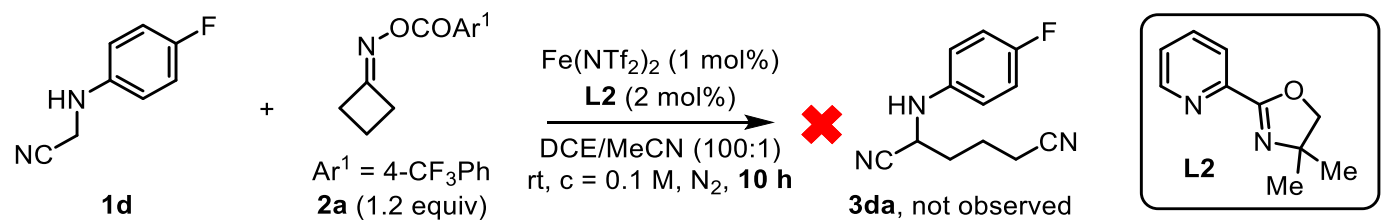

The reaction between $\mathbf{1 d}$ and $\mathbf{2 a}$ was performed under condition A. Very low conversion (for both $\mathbf{1 d}$ and 2a) was observed even after extended reaction time, and the anticipated product 3da was not observed on crude NMR. This result suggested a critical role of a neighboring carbonyl group.

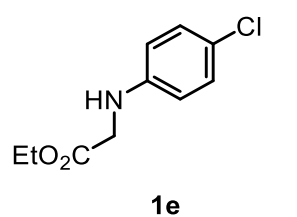

$1 \mathrm{e}$

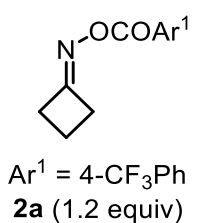

2a (1.2 equiv)

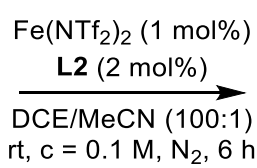

$\mathrm{rt}, \mathrm{c}=0.1 \mathrm{M}, \mathrm{N}_{2}, 6 \mathrm{~h}$
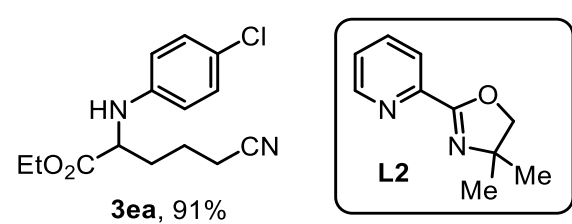

Ethyl 2-((4-chlorophenyl)amino)-5-cyanopentanoate (3ea): Under condition A, compound 3ea was prepared by reacting 1e with $\mathbf{2 a}$, and the product was isolated through a silica gel flash column (PE: EtOAc, from 10:1 to 5:1) as yellow oil (51 mg, 91\% yield). ${ }^{1} \mathrm{H}$ NMR (400 MHz, Chloroform- $d$ ) $\delta 7.18-$ $7.07(\mathrm{~m}, 2 \mathrm{H}), 6.60-6.50(\mathrm{~m}, 2 \mathrm{H}), 4.20(\mathrm{q}, J=7.1 \mathrm{~Hz}, 2 \mathrm{H}), 4.16(\mathrm{br}, 1 \mathrm{H}), 4.03(\mathrm{dt}, J=8.8,6.2 \mathrm{~Hz}, 1 \mathrm{H})$, $2.40(\mathrm{td}, J=6.9,2.9 \mathrm{~Hz}, 2 \mathrm{H}), 2.08-1.96(\mathrm{~m}, 1 \mathrm{H}), 1.91(\mathrm{dt}, J=13.9,7.1 \mathrm{~Hz}, 1 \mathrm{H}), 1.86-1.76(\mathrm{~m}, 2 \mathrm{H})$, $1.26(\mathrm{t}, J=7.1 \mathrm{~Hz}, 3 \mathrm{H}) ;{ }^{13} \mathrm{C}\left\{{ }^{1} \mathrm{H}\right\} \mathrm{NMR}(101 \mathrm{MHz}$, Chloroform- $d$ ) $\delta 173.0,145.1,129.3,123.4,119.0$, 114.8, 61.6, 56.1, 31.6, 21.7, 17.0, 14.2; IR $v_{\max }$ (neat)/cm ${ }^{-1}: 3376,2932,2246,1728,1600,1492,1181$, 1018; HRMS (ESI, m/z): calcd for $\mathrm{C}_{14} \mathrm{H}_{18} \mathrm{ClN}_{2} \mathrm{O}_{2}{ }^{+}[\mathrm{M}+\mathrm{H}]^{+}$281.1051, found 281.1053.

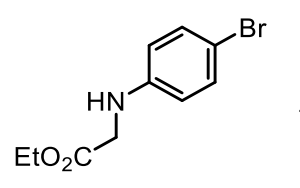

$1 \mathrm{f}$

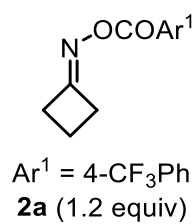

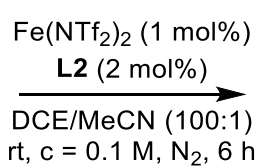
DCE/MeCN $(100: 1)$
rt, c $=0.1 \mathrm{M}, \mathrm{N}_{2}, 6 \mathrm{~h}$
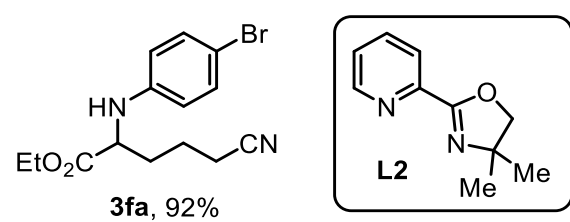

Ethyl 2-((4-bromophenyl)amino)-5-cyanopentanoate (3fa): Under condition A, compound 3fa was prepared by reacting $\mathbf{1 f}$ with $\mathbf{2 a}$, and the product was isolated through a silica gel flash column (PE: 
EtOAc, from 10:1 to 5:1) as yellow oil (60 mg, 92\% yield). ${ }^{1} \mathrm{H}$ NMR (400 MHz, Chloroform- $d$ ) $\delta 7.31-$ $7.21(\mathrm{~m}, 2 \mathrm{H}), 6.55-6.44(\mathrm{~m}, 2 \mathrm{H}), 4.22(\mathrm{br}, 1 \mathrm{H}), 4.20(\mathrm{q}, J=7.2 \mathrm{~Hz}, 2 \mathrm{H}), 4.09-3.98(\mathrm{~m}, 1 \mathrm{H}), 2.40$ (td, $J=6.9,2.9 \mathrm{~Hz}, 2 \mathrm{H}), 2.08-1.95(\mathrm{~m}, 1 \mathrm{H}), 1.91(\mathrm{dt}, J=14.0,7.1 \mathrm{~Hz}, 1 \mathrm{H}), 1.88-1.72(\mathrm{~m}, 2 \mathrm{H}), 1.26(\mathrm{t}, J$ $=7.1 \mathrm{~Hz}, 3 \mathrm{H}) ;{ }^{13} \mathrm{C}\left\{{ }^{1} \mathrm{H}\right\} \mathrm{NMR}(101 \mathrm{MHz}$, Chloroform- $d) \delta 173.0,145.5,132.1,119.0,115.2,110.4,61.6$, 56.0, 31.6, 21.7, 17.0, 14.2; IR $v_{\max }$ (neat)/cm $\mathrm{cm}^{-1}: 3376,2932,2246,1728,1593,1488,1181,1019$; HRMS (ESI, m/z): calcd for $\mathrm{C}_{14} \mathrm{H}_{18} \mathrm{BrN}_{2} \mathrm{O}_{2}{ }^{+}[\mathrm{M}+\mathrm{H}]^{+}$325.0546, found 325.0547.
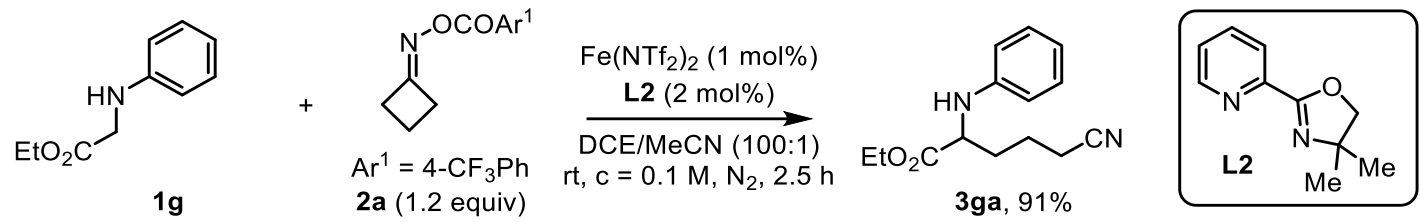

Ethyl 5-cyano-2-(phenylamino)pentanoate (3ga): Under condition A, compound 3ga was prepared by reacting $1 \mathbf{g}$ with $\mathbf{2 a}$, and the product was isolated through a silica gel flash column (PE: EtOAc, from 10:1 to 5:1) as yellowish oil (45 mg, 91\%). ${ }^{1} \mathrm{H}$ NMR (400 MHz, Chloroform- $d$ ) $\delta 7.23-7.14(\mathrm{~m}, 2 \mathrm{H}), 6.81-$ $6.72(\mathrm{~m}, 1 \mathrm{H}), 6.67-6.60(\mathrm{~m}, 2 \mathrm{H}), 4.20(\mathrm{q}, J=7.2 \mathrm{~Hz}, 2 \mathrm{H}), 4.17(\mathrm{br}, 1 \mathrm{H}), 4.09(\mathrm{q}, J=6.5 \mathrm{~Hz}, 1 \mathrm{H}), 2.40$ $(\mathrm{td}, J=6.9,2.9 \mathrm{~Hz}, 2 \mathrm{H}), 2.08-1.97(\mathrm{~m}, 1 \mathrm{H}), 1.96-1.77(\mathrm{~m}, 3 \mathrm{H}), 1.26(\mathrm{t}, J=7.1 \mathrm{~Hz}, 3 \mathrm{H}) ;{ }^{13} \mathrm{C}\left\{{ }^{1} \mathrm{H}\right\} \mathrm{NMR}$ (101 MHz, Chloroform- $d$ ) $\delta 173.3,146.5,129.4,119.1,118.7,113.6,61.5,56.0,31.8,21.8,17.0,14.2$; IR $v_{\max }\left(\right.$ neat) $/ \mathrm{cm}^{-1}: 3376,2927,2246,1728,1602,1497,1182,1020 ;$ HRMS (ESI, m/z): calcd for $\mathrm{C}_{14} \mathrm{H}_{19} \mathrm{~N}_{2} \mathrm{O}_{2}{ }^{+}[\mathrm{M}+\mathrm{H}]^{+} 247.1441$, found 247.1442 .

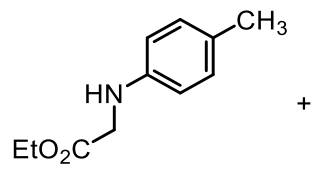

$1 \mathrm{~h}$

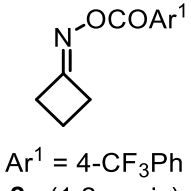

2a (1.2 equiv)

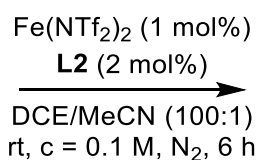

DCE/MeCN $(100: 1)$
$\mathrm{rt}, \mathrm{c}=0.1 \mathrm{M}, \mathrm{N}_{2}, 6 \mathrm{~h}$
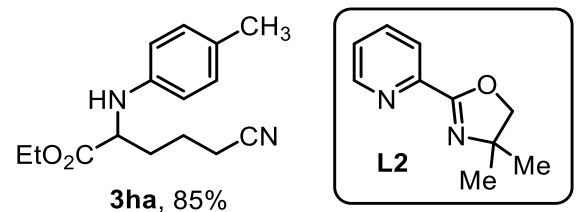

3ha, $85 \%$

Ethyl 5-cyano-2-(p-tolylamino)pentanoate (3ha): Under condition A, compound 3ha was prepared by reacting $\mathbf{1 h}$ with $\mathbf{2 a}$, and the product was isolated through a silica gel flash column (PE: EtOAc, from 10:1 to 5:1) as yellowish oil (44 mg, 85\% yield). ${ }^{1} \mathrm{H}$ NMR (400 MHz, Chloroform- $d$ ) $\delta 7.05-6.94$ (m, 2H), $6.59-6.52(\mathrm{~m}, 2 \mathrm{H}), 4.19(\mathrm{q}, J=7.1 \mathrm{~Hz}, 2 \mathrm{H}), 4.11-3.96(\mathrm{~m}, 2 \mathrm{H}), 2.39(\mathrm{td}, J=6.9,2.4 \mathrm{~Hz}, 2 \mathrm{H})$, $2.24(\mathrm{~s}, 3 \mathrm{H}), 2.06-1.96(\mathrm{~m}, 1 \mathrm{H}), 1.94-1.77(\mathrm{~m}, 3 \mathrm{H}), 1.26(\mathrm{t}, J=7.1 \mathrm{~Hz}, 3 \mathrm{H}) ;{ }^{13} \mathrm{C}\left\{{ }^{1} \mathrm{H}\right\} \mathrm{NMR}(101 \mathrm{MHz}$, Chloroform- $d$ ) $\delta 173.5,144.2,129.9,128.0,119.2,113.9,61.4,56.4,31.8,21.8,20.4,17.0,14.2$; IR $v_{\max }$ (neat) $/ \mathrm{cm}^{-1}: 3375,2922,2246,1728,1616,1519,1184,1020 ; \mathrm{HRMS}$ (ESI, m/z): calcd for $\mathrm{C}_{15} \mathrm{H}_{21} \mathrm{~N}_{2} \mathrm{O}_{2}{ }^{+}$ $[\mathrm{M}+\mathrm{H}]^{+}$261.1598, found 261.1599.
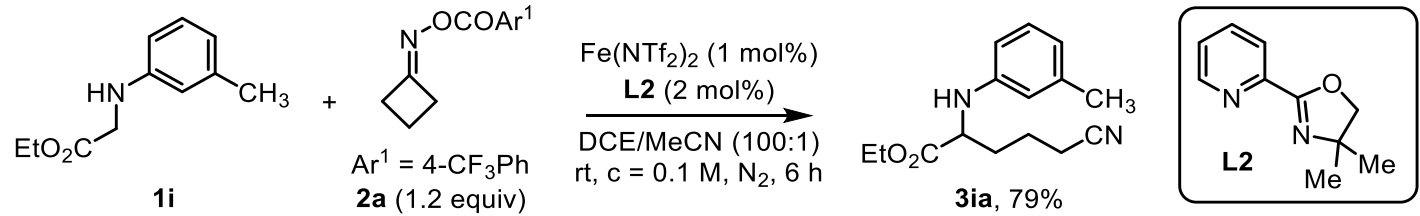
Ethyl 5-cyano-2-(m-tolylamino)pentanoate (3ia): Under condition A, compound 3ia was prepared by reacting $\mathbf{1 i}$ with $\mathbf{2 a}$, and the product was isolated through a silica gel flash column (PE: EtOAc, from 10:1 to 5:1) as a light yellow solid (41 mg, 78\% yield, m.p. $\left.72-73{ }^{\circ} \mathrm{C}\right) .{ }^{1} \mathrm{H}$ NMR (400 MHz, Chloroform- $d$ ) $\delta 7.07(\mathrm{t}, J=7.7 \mathrm{~Hz}, 1 \mathrm{H}), 6.59(\mathrm{~d}, J=7.4 \mathrm{~Hz}, 1 \mathrm{H}), 6.49-6.40(\mathrm{~m}, 2 \mathrm{H}), 4.25-4.14(\mathrm{~m}, 2 \mathrm{H}), 4.17-4.03$ $(\mathrm{m}, 2 \mathrm{H}), 2.40(\mathrm{td}, J=6.9,3.1 \mathrm{~Hz}, 2 \mathrm{H}), 2.27(\mathrm{~s}, 3 \mathrm{H}), 2.09-1.95(\mathrm{~m}, 1 \mathrm{H}), 1.96-1.75(\mathrm{~m}, 3 \mathrm{H}), 1.27(\mathrm{t}, J$ $=7.1 \mathrm{~Hz}, 3 \mathrm{H}) ;{ }^{13} \mathrm{C}\left\{{ }^{1} \mathrm{H}\right\} \mathrm{NMR}(101 \mathrm{MHz}$, Chloroform- $d) \delta 173.4,146.5,139.2,129.3,119.7,119.1,114.5$, 110.7, 61.4, 56.0, 31.8, 21.8, 21.6, 17.0, 14.2; IR $v_{\max }$ (neat) $/ \mathrm{cm}^{-1}: 3376,2924,2245,1728,1605,1590$, 1489, 1180, 1020; HRMS (ESI, m/z): calcd for $\mathrm{C}_{15} \mathrm{H}_{21} \mathrm{~N}_{2} \mathrm{O}_{2}{ }^{+}[\mathrm{M}+\mathrm{H}]^{+}$261.1598, found 261.1598.

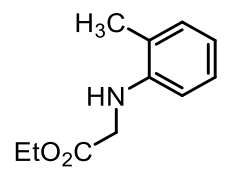

$1 \mathbf{j}$

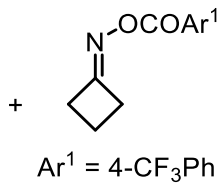

2a (1.2 equiv)

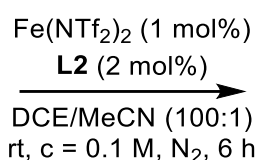

$\mathrm{rt}, \mathrm{c}=0.1 \mathrm{M}, \mathrm{N}, 6 \mathrm{~h}$

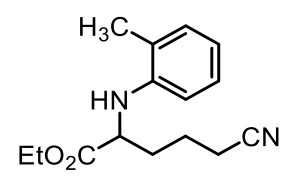

$3 \mathbf{j a}, 77 \%$

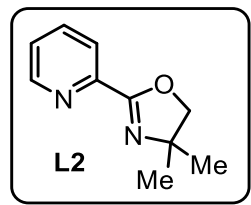

$\mathrm{Me} \mathrm{Me}$

Ethyl 5-cyano-2-(o-tolylamino)pentanoate (3ja): Under condition A, compound 3ja was prepared by reacting $\mathbf{1 j}$ with $\mathbf{2 a}$, and the product was isolated through a silica gel flash column (PE: EtOAc, from 10:1 to 5:1) as yellowish oil (40 mg, 77\% yield). ${ }^{1} \mathrm{H}$ NMR (400 MHz, Chloroform- $d$ ) $\delta 7.14-7.05$ (m, 2H), $6.71(\mathrm{t}, J=7.3,1 \mathrm{H}), 6.54(\mathrm{~d}, J=8.0 \mathrm{~Hz}, 1 \mathrm{H}), 4.21(\mathrm{q}, J=7.1 \mathrm{~Hz}, 2 \mathrm{H}), 4.15$ (t, $J=6.2 \mathrm{~Hz}, 1 \mathrm{H}), 4.09$ (brs, $1 \mathrm{H}), 2.49-2.32(\mathrm{~m}, 2 \mathrm{H}), 2.22(\mathrm{~s}, 3 \mathrm{H}), 2.12-2.00(\mathrm{~m}, 1 \mathrm{H}), 2.00-1.90(\mathrm{~m}, 1 \mathrm{H}), 1.89-1.79(\mathrm{~m}, 2 \mathrm{H})$, $1.28(\mathrm{t}, J=7.1 \mathrm{~Hz}, 3 \mathrm{H}) ;{ }^{13} \mathrm{C}\left\{{ }^{1} \mathrm{H}\right\} \mathrm{NMR}\left(101 \mathrm{MHz}, \mathrm{CDCl}_{3}\right) \delta 173.5,144.5,130.5,127.1,123.1,119.2$, 118.3, 110.4, 61.5, 55.9, 31.8, 21.8, 17.5, 17.1, 14.2; IR $v_{\max }$ (neat) $/ \mathrm{cm}^{-1}: 3403,2979,2937,2874,2246$, 1734, 1606, 1588, 1515, 1452, 1315, 1182, 1021, 751; HRMS (ESI, m/z): calcd for $\mathrm{C}_{15} \mathrm{H}_{21} \mathrm{~N}_{2} \mathrm{O}_{2}{ }^{+}[\mathrm{M}+$ $\mathrm{H}]^{+} 261.1598$, found 261.1601 .

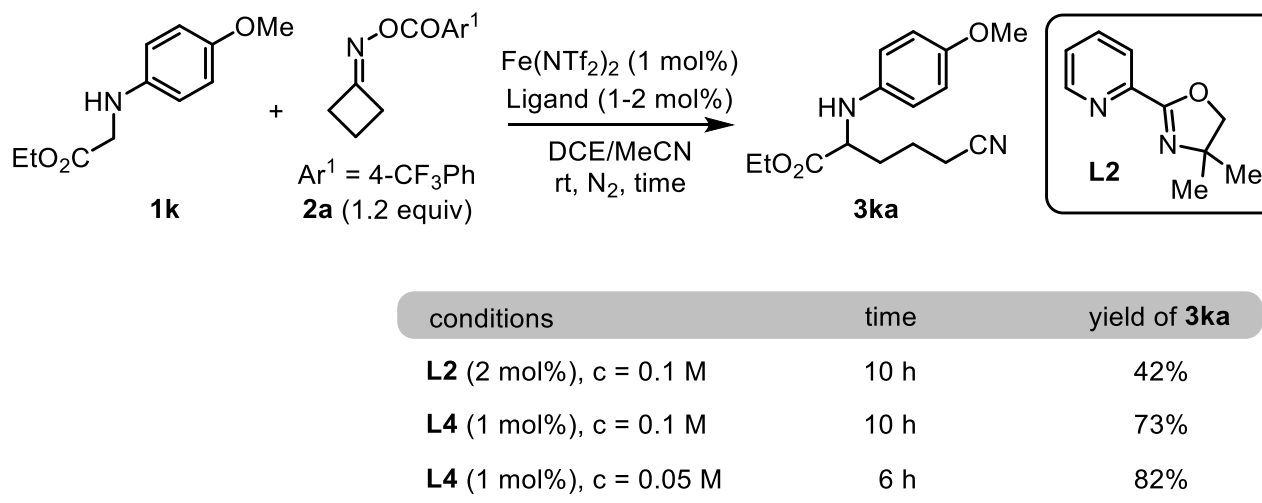

When glycinate 1k and oxime ester $\mathbf{2 a}$ were treated with $\mathrm{Fe}\left(\mathrm{NTf}_{2}\right)_{2} / \mathbf{L} \mathbf{2}$ under the standard conditions, the reaction proceeded slowly and generated more side products. After a brief optimization, a satisfactory yield was achieved by using a tridentate pyridine-bisoxazoline ligand L4 with lower reaction concentration for this electron-rich substrate, and the new experimental procedure is summarized below as condition $\mathrm{B}$. 
b) Condition B: $\mathrm{Fe}\left(\mathrm{NTf}_{2}\right)_{2} / \mathbf{L} 4(1: 1)$ as the catalyst

General procedures: In a $\mathrm{N}_{2}$ atmosphere glovebox, $\mathrm{Fe}\left(\mathrm{NTf}_{2}\right)_{2}(24.6 \mathrm{mg}, 0.04 \mathrm{mmol})$, and $\mathbf{L 4}(11.0 \mathrm{mg}$, $0.04 \mathrm{mmol}$ ) were weighed into a 2-dram vial charged with a stir bar. The vial was sealed under $\mathrm{N}_{2}$ with a septum and taken out of the glovebox. Then, freshly distilled dry 1,2-dichloroethane $(3.2 \mathrm{~mL})$ and dry $\mathrm{MeCN}$ ( $0.8 \mathrm{~mL}$, note: this complex requires more $\mathrm{MeCN}$ to dissolve) was added into the vial. The mixture was stirred at room temperature to afford a red catalyst solution (c=0.01 M, $0.2 \mathrm{ml}$ for each $0.2 \mathrm{mmol}$ scale reaction). Outside the glovebox, to another flame-dried sealable 2-dram vial charged with a stir bar were added a corresponding $N$-PMP glycinate $\mathbf{1}(0.2 \mathrm{mmol})$ and oxime ester $\mathbf{2}(0.24 \mathrm{mmol})$. After the vial was evacuated and backfilled with $\mathrm{N}_{2}$ three times, anhydrous 1,2-dichloroethane $(3.8 \mathrm{~mL})$ was added, followed by the addition of catalyst solution $(0.2 \mathrm{~mL})$. The resulting mixture was stirred at room temperature $\left(23\right.$ to $\left.26^{\circ} \mathrm{C}\right)$ and the reaction progress was monitored by TLC. Normally, a white precipitate (4-trifluoromethyl benzoic acid) quickly forms as the oxime ester decomposes. After the indicated time, the reaction was quenched with saturated $\mathrm{NaHCO}_{3}$ aqueous solution $(2 \mathrm{~mL})$ and the aqueous phase was extracted with $\mathrm{CH}_{2} \mathrm{Cl}_{2}(1 \mathrm{~mL} \times 3)$. The combined organic layers were dried over anhydrous $\mathrm{Na}_{2} \mathrm{SO}_{4}$, filtered, and then concentrated in vacuo to afford the crude product, which was purified through a silica gel flash column with PE and EtOAc as eluent.

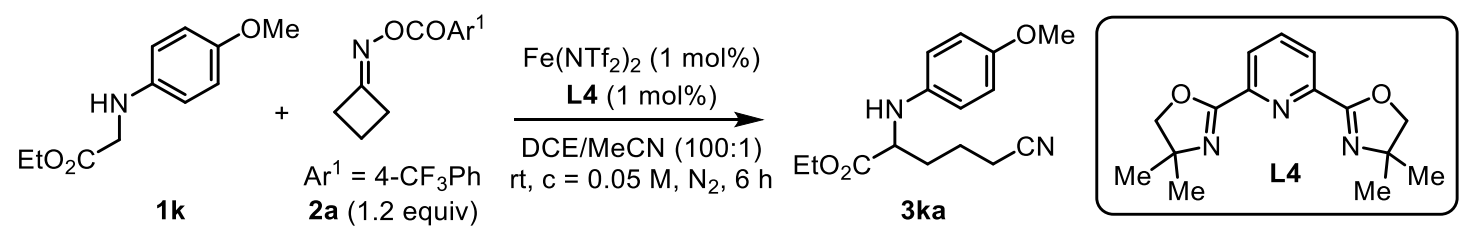

Ethyl 5-cyano-2-((4-methoxyphenyl)amino)pentanoate (3ka): Under condition A, compound 3ba was prepared by reacting $\mathbf{1 i}$ with $\mathbf{2 a}$, and the product was isolated through a silica gel flash column (PE: EtOAc, from 10:1 to 5:1) as yellowish solid (45 mg, 82\% yield, m.p. 68-69 $\left.{ }^{\circ} \mathrm{C}\right) .{ }^{1} \mathrm{H}$ NMR (400 MHz, Chloroformd) $\delta 6.80-6.73(\mathrm{~m}, 2 \mathrm{H}), 6.64-6.56(\mathrm{~m}, 2 \mathrm{H}), 4.17(\mathrm{q}, J=7.1 \mathrm{~Hz}, 2 \mathrm{H}), 4.03-3.94(\mathrm{~m}, 1 \mathrm{H}), 3.73(\mathrm{~s}, 3 \mathrm{H})$, $3.61(\mathrm{br}, 1 \mathrm{H}), 2.40(\mathrm{td}, J=6.8,1.8 \mathrm{~Hz}, 2 \mathrm{H}), 2.03-1.92(\mathrm{~m}, 1 \mathrm{H}), 1.92-1.76(\mathrm{~m}, 3 \mathrm{H}), 1.24(\mathrm{t}, J=7.1 \mathrm{~Hz}$, $3 \mathrm{H}) ;{ }^{13} \mathrm{C}\left\{{ }^{1} \mathrm{H}\right\} \mathrm{NMR}(101 \mathrm{MHz}$, Chloroform- $d) \delta 173.7,153.0,140.6,119.2,115.4,114.9,61.3,57.2,55.7$, 31.9, 21.9, 17.0, 14.2; IR $v_{\max }$ (neat)/(cm ${ }^{-1}: 3364,2935,2245,1727,1511,1180,1031 ; \mathrm{HRMS}$ (ESI, m/z): calcd for $\mathrm{C}_{15} \mathrm{H}_{21} \mathrm{~N}_{2} \mathrm{O}_{3}{ }^{+}[\mathrm{M}+\mathrm{H}]^{+} 277.1547$, found 277.1550 .
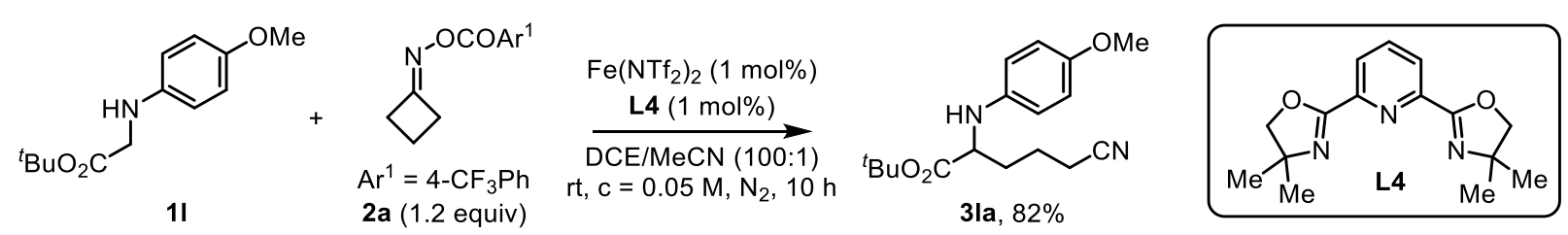

tert-Butyl 5-cyano-2-((4-methoxyphenyl)amino)pentanoate (3la): Under condition B, compound 3la was prepared by reacting $\mathbf{1 1}$ with $\mathbf{2 a}$, and the product was isolated through a silica gel flash column (PE: 
EtOAc, from 10:1 to 5:1) as yellow solid (50 mg, 82\% yield, m.p. $\left.46-47{ }^{\circ} \mathrm{C}\right)$; ${ }^{1} \mathrm{H} \mathrm{NMR}(400 \mathrm{MHz}$, Chloroform- $d$ ) $\delta 6.79-6.74(\mathrm{~m}, 2 \mathrm{H}), 6.64-6.56(\mathrm{~m}, 2 \mathrm{H}), 3.92-3.83(\mathrm{~m}, 2 \mathrm{H}), 3.74(\mathrm{~s}, 3 \mathrm{H}), 2.40(\mathrm{td}, J$ $=6.8,3.9 \mathrm{~Hz}, 2 \mathrm{H}), 2.01-1.90(\mathrm{~m}, 1 \mathrm{H}), 1.91-1.73(\mathrm{~m}, 3 \mathrm{H}), 1.42(\mathrm{~s}, 9 \mathrm{H}) ;{ }^{13} \mathrm{C}\left\{{ }^{1} \mathrm{H}\right\} \mathrm{NMR}(101 \mathrm{MHz}$, Chloroform- $d$ ) $\delta 172.8,152.9,140.8,119.3,115.3,114.9,82.0,57.7,55.7,31.8,28.0,21.8,17.1$; IR $v_{\max }$ (neat) $/ \mathrm{cm}^{-1}: 3360,2933,2246,1724,1511,1367,1236,1149,1035 ;$ HRMS (ESI, m/z): calcd for $\mathrm{C}_{17} \mathrm{H}_{25} \mathrm{~N}_{2} \mathrm{O}_{3}{ }^{+}[\mathrm{M}+\mathrm{H}]^{+} 305.1860$, found 305.1862.
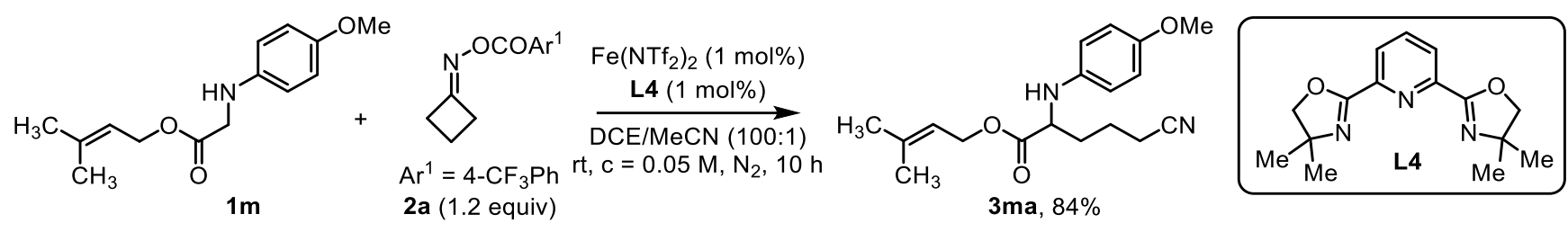

3-Prenyl 5-cyano-2-((4-methoxyphenyl)amino)pentanoate (3ma): Under condition $\mathrm{B}$, compound 3ma was prepared by reacting $\mathbf{1 m}$ with $\mathbf{2 a}$, and the product was isolated through a silica gel flash column (PE: EtOAc, from 10:1 to 5:1) as yellowish oil (53 mg, 84\%); ${ }^{1} \mathrm{H}$ NMR (400 MHz, Chloroform- $d$ ) $\delta 6.80-$ $6.72(\mathrm{~m}, 2 \mathrm{H}), 6.64-6.56(\mathrm{~m}, 2 \mathrm{H}), 5.34-5.25(\mathrm{~m}, 1 \mathrm{H}), 4.68-4.52(\mathrm{~m}, 2 \mathrm{H}), 4.02-3.95(\mathrm{~m}, 1 \mathrm{H}), 3.73$ (s, 3H), $2.44-2.35(\mathrm{~m}, 2 \mathrm{H}), 2.03-1.93(\mathrm{~m}, 1 \mathrm{H}), 1.92-1.77(\mathrm{~m}, 3 \mathrm{H}), 1.75(\mathrm{~s}, 3 \mathrm{H}), 1.68(\mathrm{~s}, 3 \mathrm{H})$; ${ }^{13} \mathrm{C}\left\{{ }^{1} \mathrm{H}\right\} \mathrm{NMR}\left(101 \mathrm{MHz}, \mathrm{CDCl}_{3}\right) \delta 173.7,153.0,140.6,140.2,119.2,117.9,115.4,114.9,62.1,57.3$, 55.7, 31.9, 25.7, 21.9, 18.0, 17.0; IR $v_{\max }$ (neat)/cm $\mathrm{cm}^{-1}: 3366,2937,2834,2246,1733,1514,1457,1380$, 1239, 1179, 1037, 938, 823; HRMS (ESI, m/z): calcd for $\mathrm{C}_{18} \mathrm{H}_{25} \mathrm{~N}_{2} \mathrm{O}_{3}{ }^{+}[\mathrm{M}+\mathrm{H}]^{+} 317.1860$, found 317.1858 .
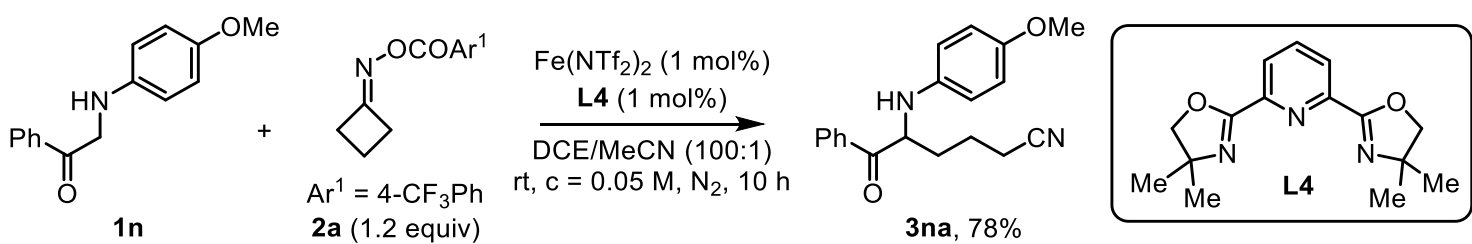

5-((4-Methoxyphenyl)amino)-6-oxo-6-phenylhexanenitrile (3na): Under condition B, compound 3na was prepared by reacting $\mathbf{1 n}$ with $\mathbf{2 a}$, and the product was isolated through a silica gel flash column (PE: EtOAc, from 10:1 to 4:1) as yellowish oil (48 mg, 78\% yield). ${ }^{1} \mathrm{H}$ NMR (400 MHz, Chloroform- $d$ ) $\delta 8.01$ - $7.94(\mathrm{~m}, 2 \mathrm{H}), 7.67-7.56(\mathrm{~m}, 1 \mathrm{H}), 7.55-7.46(\mathrm{~m}, 2 \mathrm{H}), 6.82-6.72(\mathrm{~m}, 2 \mathrm{H}), 6.71-6.61(\mathrm{~m}, 2 \mathrm{H}), 5.07$ - $4.95(\mathrm{~m}, 1 \mathrm{H}), 4.43(\mathrm{br}, 1 \mathrm{H}), 3.72(\mathrm{~s}, 3 \mathrm{H}), 2.35(\mathrm{t}, J=6.8 \mathrm{~Hz}, 2 \mathrm{H}), 2.21-2.02(\mathrm{~m}, 1 \mathrm{H}), 1.92-1.70(\mathrm{~m}$, $3 \mathrm{H}) ;{ }^{13} \mathrm{C}\left\{{ }^{1} \mathrm{H}\right\} \mathrm{NMR}(101 \mathrm{MHz}$, Chloroform- $d) \delta 200.4,152.9,140.9,134.8,133.9,129.1,128.3,119.2$, 115.5, 115.0, 58.5, 55.7, 32.0, 21.5, 17.1; IR $v_{\max }$ (neat)/( $\mathrm{cm}^{-1}: 3360,2925,2245,1682,1509,1447,1236$, 1177, 1034; HRMS (ESI, m/z): calcd for $\mathrm{C}_{19} \mathrm{H}_{21} \mathrm{~N}_{2} \mathrm{O}_{2}{ }^{+}[\mathrm{M}+\mathrm{H}]^{+}$309.1598, found 309.1599. 
<smiles>COc1ccc(NCC(=O)Nc2ccccc2)cc1</smiles>

10

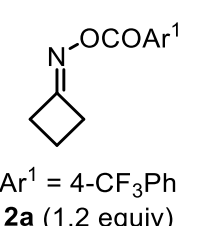

2a (1.2 equiv)

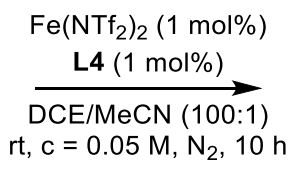
$\mathrm{rt}, \mathrm{c}=0.05 \mathrm{M}, \mathrm{N}_{2}, 10 \mathrm{~h}$

3oa, $82 \%$
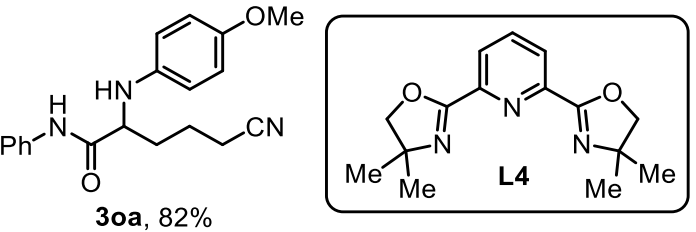

5-Cyano-2-((4-methoxyphenyl)amino)-N-phenylpentanamide (3oa): Under condition $\mathrm{B}$, compound 3oa was prepared by reacting 10 with $\mathbf{2 a}$, and the product was isolated through a silica gel flash column (PE: EtOAc, from 5:1 to 3:1) as yellow solid (53 mg, 82\% yield, mp: $\left.118-119{ }^{\circ} \mathrm{C}\right) .{ }^{1} \mathrm{H} \mathrm{NMR}(400 \mathrm{MHz}$, Chloroform- $d$ ) $\delta 8.82$ (brs, 1H), $7.57-7.49$ (m, 2H), $7.34-7.27$ (m, 2H), $7.15-7.07$ (m, 1H), $6.84-$ $6.76(\mathrm{~m}, 2 \mathrm{H}), 6.70-6.58(\mathrm{~m}, 2 \mathrm{H}), 3.88(\mathrm{br}, 1 \mathrm{H}), 3.74(\mathrm{~s}, 3 \mathrm{H}), 3.73-3.68(\mathrm{~m}, 1 \mathrm{H}), 2.50-2.33(\mathrm{~m}, 2 \mathrm{H})$, $2.21-2.08(\mathrm{~m}, 1 \mathrm{H}), 2.02-1.82(\mathrm{~m}, 3 \mathrm{H}) ;{ }^{13} \mathrm{C}\left\{{ }^{1} \mathrm{H}\right\} \mathrm{NMR}(101 \mathrm{MHz}$, Chloroform- $d) \delta 171.2,153.7,140.1$, 137.2, 129.0, 124.6, 119.9, 119.3, 115.2, 115.1, 60.6, 55.7, 32.6, 22.3, 17.1; IR $v_{\max }\left(\right.$ neat) $/ \mathrm{cm}^{-1}: 3331$, 2935, 2246, 1668, 1599, 1510, 1442, 1237, 1178, 1035; HRMS (ESI, m/z): calcd for $\mathrm{C}_{19} \mathrm{H}_{22} \mathrm{~N}_{3} \mathrm{O}_{2}{ }^{+}[\mathrm{M}+$ $\mathrm{H}]^{+} 324.1707$, found 324.1709 .
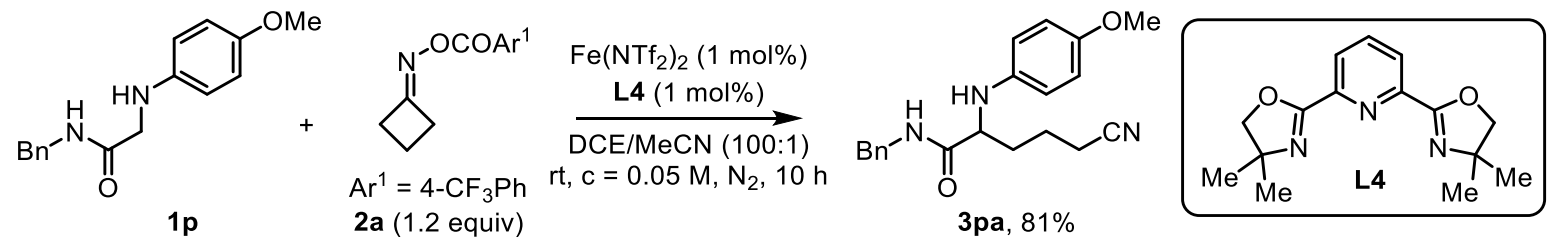

N-Benzyl-5-cyano-2-((4-methoxyphenyl)amino)pentanamide (3pa): Under condition B, compound 3pa was prepared by reacting 1p with $\mathbf{2 a}$, and the product was isolated through a silica gel flash column (PE: EtOAc, from 5:1 to 3:1) as yellowish oil (55 mg, 81\% yield). ${ }^{1} \mathrm{H}$ NMR (400 MHz, Chloroform- $d$ ) $\delta 7.31$ $-7.20(\mathrm{~m}, 4 \mathrm{H}), 7.17-7.12(\mathrm{~m}, 2 \mathrm{H}), 6.81-6.73(\mathrm{~m}, 2 \mathrm{H}), 6.61-6.53(\mathrm{~m}, 2 \mathrm{H}), 4.47(\mathrm{dd}, J=14.9,6.1 \mathrm{~Hz}$, 1H), 4.37 (dd, $J=14.9,5.8 \mathrm{~Hz}, 1 \mathrm{H}), 3.77$ (br, 1H), 3.74 (s, 3H), 3.69 (t, $J=6.4 \mathrm{~Hz}, 1 \mathrm{H}), 2.39$ (td, $J=6.8$, $4.8 \mathrm{~Hz}, 2 \mathrm{H}), 2.14-2.02(\mathrm{~m}, 1 \mathrm{H}), 1.96-1.76(\mathrm{~m}, 3 \mathrm{H}) ;{ }^{13} \mathrm{C}\left\{{ }^{1} \mathrm{H}\right\} \mathrm{NMR}(101 \mathrm{MHz}$, Chloroform- $d) \delta 172.8$, 153.4, 140.3, 138.0, 128.7, 127.51, 127.47, 119.3, 115.0 (4C), 59.7, 55.7, 43.2, 32.6, 22.3, 17.0; IR $v_{\max }$ (neat)/cm ${ }^{-1}: 3340,2931,2832,2246,1645,1510,1454,1235,1179,1034 ;$ HRMS (ESI, m/z): calcd for $\mathrm{C}_{20} \mathrm{H}_{24} \mathrm{~N}_{3} \mathrm{O}_{2}^{+}[\mathrm{M}+\mathrm{H}]^{+}$338.1863, found 338.1865.

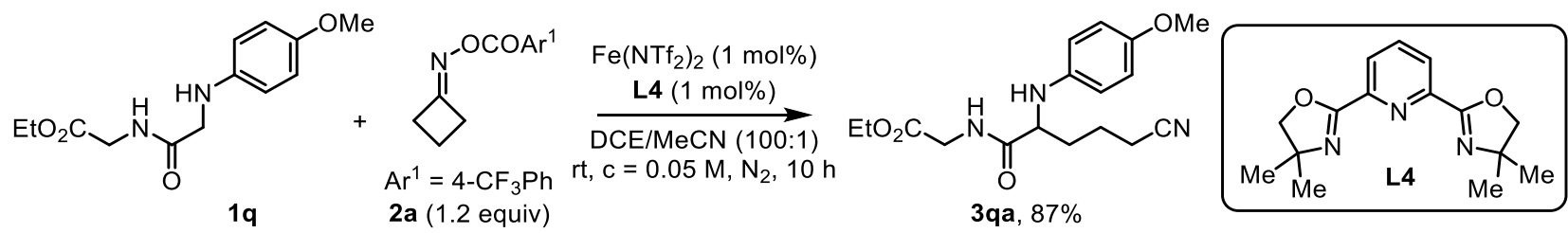

Ethyl (5-cyano-2-((4-methoxyphenyl)amino)pentanoyl)glycinate (3qa): Under condition B, compound 3qa was prepared by reacting 1q with $\mathbf{2 a}$, and the product was isolated through a silica gel flash column (PE: EtOAc, from 4:1 to 2:1) as yellowish oil (58 mg, 87\% yield). ${ }^{1} \mathrm{H}$ NMR (400 MHz, Chloroform- $d$ ) $\delta$ $7.34(\mathrm{t}, J=5.8 \mathrm{~Hz}, 1 \mathrm{H}), 6.82-6.72(\mathrm{~m}, 2 \mathrm{H}), 6.63-6.53(\mathrm{~m}, 2 \mathrm{H}), 4.15(\mathrm{q}, J=7.2 \mathrm{~Hz}, 2 \mathrm{H}), 4.09(\mathrm{dd}, J=$ 18.1, $6.3 \mathrm{~Hz}, 1 \mathrm{H}), 3.88(\mathrm{dd}, J=18.1,5.1 \mathrm{~Hz}, 1 \mathrm{H}), 3.80(\mathrm{br}, 1 \mathrm{H}), 3.72(\mathrm{~s}, 3 \mathrm{H}), 3.68(\mathrm{t}, J=6.3 \mathrm{~Hz}, 1 \mathrm{H})$, 
$2.40(\mathrm{td}, J=6.9,2.0 \mathrm{~Hz}, 2 \mathrm{H}), 2.13-2.02(\mathrm{~m}, 1 \mathrm{H}), 1.99-1.80(\mathrm{~m}, 3 \mathrm{H}), 1.24(\mathrm{t}, J=7.1 \mathrm{~Hz}, 3 \mathrm{H})$; ${ }^{13} \mathrm{C}\left\{{ }^{1} \mathrm{H}\right\}$ NMR (101 MHz, Chloroform- $d$ ) $\delta 173.4,169.5,153.3,140.4,119.3,114.99,114.95,61.5,59.5$,

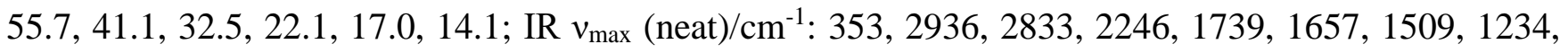
1199, 1031; HRMS (ESI, m/z): calcd for $\mathrm{C}_{17} \mathrm{H}_{24} \mathrm{~N}_{3} \mathrm{O}_{4}{ }^{+}[\mathrm{M}+\mathrm{H}]^{+} 334.1761$, found 334.1764.
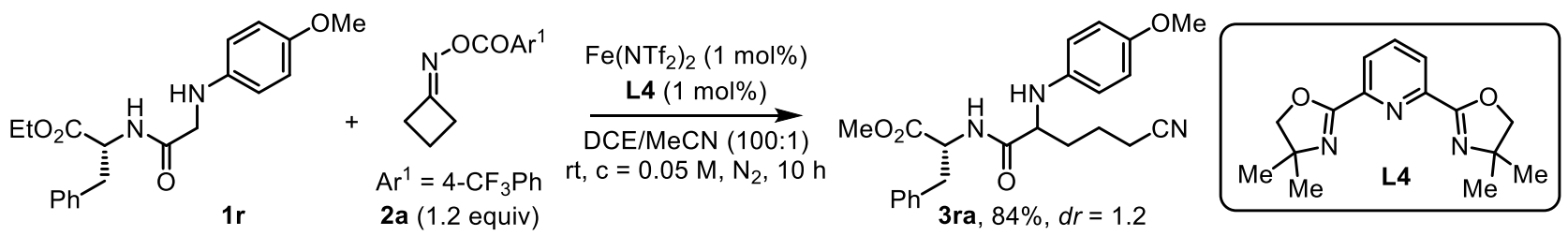

Methyl (5-cyano-2-((4-methoxyphenyl)amino)pentanoyl)-D-phenylalaninate (3ra): Under condition B, compound 3ra was prepared by reacting 1r with 2a, and the product was isolated through a silica gel flash column (PE: EtOAc, from 2:1 to 1:1) as yellowish oil (69 mg, 84\% yield, $d r=1.2) .{ }^{1} \mathrm{H}$ NMR (400 $\mathrm{MHz}$, Chloroform- $d$ ) (mixture of 2 diastereomers, 1.2:1) $\delta 7.33-7.18(\mathrm{~m}, 2 \mathrm{H}$, isomer $1+2 \mathrm{H}$, isomer 2), $7.20-7.11(\mathrm{~m}, 1 \mathrm{H}$, isomer $1+1 \mathrm{H}$, isomer 2$), 7.13-7.02(\mathrm{~m}, 2 \mathrm{H}$, isomer $1+2 \mathrm{H}$, isomer 2$), 6.85-6.72$ $(\mathrm{m}, 3 \mathrm{H}$, isomer $1+3 \mathrm{H}$, isomer 2), $6.60-6.50(\mathrm{~m}, 1 \mathrm{H}$, isomer 1), $6.53-6.44(\mathrm{~m}, 1 \mathrm{H}$, isomer 2), $4.97-$ $4.80(\mathrm{~m}, 1 \mathrm{H}$, isomer $1+1 \mathrm{H}$, isomer 2), $3.77(\mathrm{~s}, 3 \mathrm{H}$, isomer 2$), 3.74(\mathrm{~s}, 3 \mathrm{H}$, isomer 1$), 3.71(\mathrm{~s}, 3 \mathrm{H}$, isomer 2), $3.66(\mathrm{~s}, 3 \mathrm{H}$, isomer 1), $3.63(\mathrm{t}, J=6.5 \mathrm{~Hz}, 1 \mathrm{H}$, isomer 2), $3.58(\mathrm{t}, J=6.5 \mathrm{~Hz}, 1 \mathrm{H}$, isomer 1), 3.22 (dd, $J=14.1,5.3 \mathrm{~Hz}, 1 \mathrm{H}$, isomer 1), $3.04-2.91(\mathrm{~m}, 1 \mathrm{H}$, isomer $1+2 \mathrm{H}$, isomer 2), $2.42-2.33(\mathrm{~m}, 2 \mathrm{H}$, isomer 2), $2.33-2.23(\mathrm{~m}, 2 \mathrm{H}$, isomer 1$), 2.05-1.93(\mathrm{~m}, 1 \mathrm{H}$, isomer 2$), 1.91-1.70(\mathrm{~m}, 2 \mathrm{H}$, isomer $1+1 \mathrm{H}$, isomer 2), $1.65-1.51(\mathrm{~m}, 1 \mathrm{H}$, isomer $1+1 \mathrm{H}$, isomer 2$) ;{ }^{13} \mathrm{C}\left\{{ }^{1} \mathrm{H}\right\} \mathrm{NMR}(101 \mathrm{MHz}$, Chloroform- $d) \delta 172.7$, 172.6, 171.7, 171.5, 153.4, 153.3, 140.3, 140.2, 136.0, 135.3, 129.2, 129.1, 128.6 (isomer 1+ isomer 2), 127.1, 127.0, 119.24, 119.20, 115.4, 115.0, 114.83, 114.75, 59.7, 59.1, 55.8, 55.7, 52.7, 52.38, 52.35 (isomer $1+$ isomer 2), 37.8, 37.8, 32.5, 32.4, 21.9, 21.8, 17.01, 16.99; IR $v_{\max }$ (neat)/cm ${ }^{-1}: 3352,2951$, 2823, 2246, 1739, 1657, 1510, 1455, 1235, 1178, 1034; HRMS (ESI, m/z): calcd for $\mathrm{C}_{23} \mathrm{H}_{28} \mathrm{~N}_{3} \mathrm{O}_{4}{ }^{+}[\mathrm{M}+$ $\mathrm{H}]^{+}$410.2074, found 410.2075 .
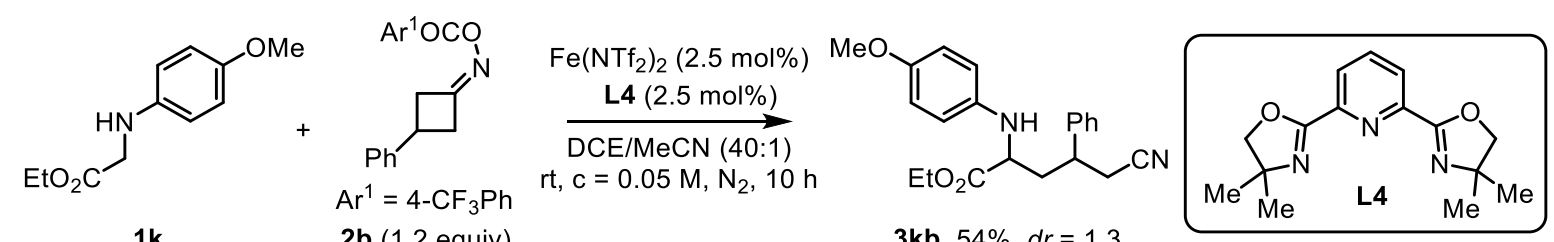

3kb, $54 \%, d r=1.3$

Ethyl 5-cyano-2-((4-methoxyphenyl)amino)-4-phenylpentanoate (3kb): Under condition B, compound $3 \mathbf{k b}$ was prepared by reacting $1 \mathbf{k}$ with $2 \mathbf{b}$ in the presence of $2.5 \mathrm{~mol} \%$ catalysts (the solvent ratio changed accordingly), and the product was isolated through a silica gel flash column (PE: EtOAc, from 10:1 to 5:1) as yellow oil (38 mg, 84\% yield, $d r=1.3$ ). ${ }^{1} \mathrm{H}$ NMR (400 MHz, Chloroform- $d$ ) (mixture of 2 diastereomers, $1.3: 1) \delta 7.42-7.36(\mathrm{~m}, 2 \mathrm{H}$, isomer $1+2 \mathrm{H}$, isomer 2$), 7.36-7.31(\mathrm{~m}, 1 \mathrm{H}$, isomer $1+1 \mathrm{H}$, isomer 2), $7.30-7.25(\mathrm{~m}, 2 \mathrm{H}$, isomer $1+2 \mathrm{H}$, isomer 2$), 6.75-6.71(\mathrm{~m}, 1 \mathrm{H}$, isomer 1$), 6.71-6.67(\mathrm{~m}$, 
$1 \mathrm{H}$, isomer 2), $6.48-6.44(\mathrm{~m}, 1 \mathrm{H}$, isomer 1), $6.44-6.40(\mathrm{~m}, 1 \mathrm{H}$, isomer 2), $4.18-4.01(\mathrm{~m}, 2 \mathrm{H}$, isomer $1+2 \mathrm{H}$, isomer 2), $3.86(\mathrm{br}, 1 \mathrm{H}$, isomer $1+1 \mathrm{H}$, isomer 2$), 3.73(\mathrm{~s}, 3 \mathrm{H}$, isomer 1$), 3.71(\mathrm{~s}, 3 \mathrm{H}$, isomer 2$)$, $3.69(\mathrm{br}, 1 \mathrm{H}$, isomer $1+1 \mathrm{H}$, isomer 2), $3.41(\mathrm{ddt}, J=11.3,6.9,3.6 \mathrm{~Hz}, 1 \mathrm{H}$, isomer 2), $3.23(\mathrm{dq}, J=8.5$, $6.7 \mathrm{~Hz}, 1 \mathrm{H}$, isomer 1), $2.75-2.60(\mathrm{~m}, 2 \mathrm{H}$, isomer $1+2 \mathrm{H}$, isomer 2$), 2.34-2.21(\mathrm{~m}, 2 \mathrm{H}$, isomer $1+1 \mathrm{H}$, isomer 2), $2.18-2.08(\mathrm{~m}, 1 \mathrm{H}$, isomer 2), $1.23(\mathrm{t}, J=7.2 \mathrm{~Hz}, 3 \mathrm{H}$, isomer 1$), 1.18(\mathrm{t}, J=7.1 \mathrm{~Hz}, 3 \mathrm{H}$, isomer 2); ${ }^{13} \mathrm{C}\left\{{ }^{1} \mathrm{H}\right\} \mathrm{NMR}(101 \mathrm{MHz}$, Chloroform- $d) \delta$ 174.0, 173.4, 153.1, 153.0, 140.8, 140.6, 140.19, 140.15, 129.2, 129.1, 128.0 (isomer 1+ isomer 2), 127.5, 127.4, 118.3, 118.1, 116.0, 115.3, 114.9, 114.7, 61.4, 61.2, 56.4, 55.7 (isomer 1+ isomer 2), 55.6, 38.9, 38.7, 38.1, 38.0, 25.3, 25.0, 14.20, 14.18; IR $v_{\max }$ (neat)/cm $\mathrm{cm}^{-1}: 3358,2934,2833,2245,1730,1512,1454,1237,1189,1134,1033 ;$ HRMS (ESI, m/z): calcd for $\mathrm{C}_{21} \mathrm{H}_{25} \mathrm{~N}_{2} \mathrm{O}_{3}{ }^{+}[\mathrm{M}+\mathrm{H}]^{+}$353.1860, found 353.1859.

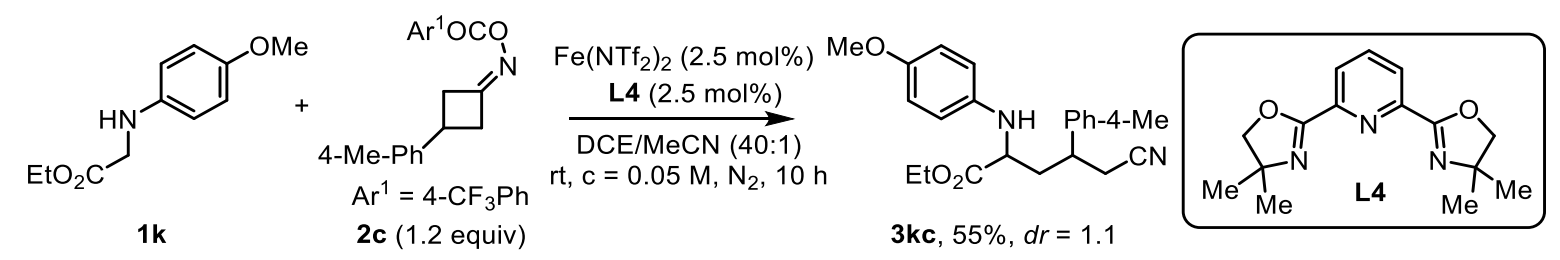

Ethyl 5-cyano-2-((4-methoxyphenyl)amino)-4-(p-tolyl)pentanoate (3kc): Under condition B, compound $3 \mathbf{k c}$ was prepared by reacting $1 \mathbf{k}$ with $2 \mathbf{c}$ in the presence of $2.5 \mathrm{~mol} \%$ catalysts (the solvent ratio changed accordingly), and the product was isolated through a silica gel flash column (PE: EtOAc, from 10:1 to 5:1) as yellowish oil (40 mg, 55\% yield, $d r=1.1) .{ }^{1} \mathrm{H}$ NMR (400 MHz, Chloroform- $d$ ) (mixture of 2 diastereomers, 1.1:1) $\delta 7.23-7.11(\mathrm{~m}, 4 \mathrm{H}$, isomer $1+4 \mathrm{H}$, isomer 2$), 6.77-6.66(\mathrm{~m}, 2 \mathrm{H}$, isomer $1+2 \mathrm{H}$, isomer 2), $6.51-6.39(\mathrm{~m}, 2 \mathrm{H}$, isomer $1+2 \mathrm{H}$, isomer 2), $4.17-4.02(\mathrm{~m}, 2 \mathrm{H}$, isomer $1+2 \mathrm{H}$, isomer 2$)$, $3.89-3.82(\mathrm{~m}, 1 \mathrm{H}$, isomer $1+1 \mathrm{H}$, isomer 2), $3.73(\mathrm{~s}, 3 \mathrm{H}$, isomer 1), $3.72(\mathrm{~s}, 3 \mathrm{H}$, isomer 2), $3.69(\mathrm{br}, 1 \mathrm{H}$, isomer $1+1 \mathrm{H}$, isomer 2), $3.37(\mathrm{ddt}, J=11.1,6.9,3.4 \mathrm{~Hz}, 1 \mathrm{H}$, isomer 2), $3.25-3.13(\mathrm{~m}, 1 \mathrm{H}$, isomer 1), $2.73-2.57(\mathrm{~m}, 2 \mathrm{H}$, isomer $1+2 \mathrm{H}$, isomer 2$), 2.36(\mathrm{~s}, 3 \mathrm{H}$, isomer $1+3 \mathrm{H}$, isomer 2$), 2.30-2.18(\mathrm{~m}, 2 \mathrm{H}$, isomer $1+1 \mathrm{H}$, isomer 2), $2.15-2.02(\mathrm{~m}, 1 \mathrm{H}$, isomer 2$), 1.23(\mathrm{t}, J=7.1 \mathrm{~Hz}, 3 \mathrm{H}$, isomer 1$), 1.18(\mathrm{t}, J=$ 7.1 Hz, 3H, isomer 2); ${ }^{13} \mathrm{C}\left\{{ }^{1} \mathrm{H}\right\} \mathrm{NMR}$ (101 MHz, Chloroform- $d$ ) $\delta$ 174.0, 173.4, 153.1, 153.0, 140.9, 140.2, 137.60, 137.58, 137.5, 137.1, 129.8, 129.7, 127.31, 127.25, 118.4, 118.2, 116.0, 115.3, 114.9, 114.7, 61.3, 61.2, 56.4, 55.7, 55.7, 55.6, 38.5, 38.32, 38.26, 38.1, 25.4, 25.1, 21.1 (isomer 1 + isomer 2), 14.20, 14.17; IR $v_{\max }\left(\right.$ neat) $/ \mathrm{cm}^{-1}: 3352,2926,2825,2246,1731,1507,1443,1238,1182,1134,1035$; HRMS (ESI, $\mathrm{m} / \mathrm{z}$ ): calcd for $\mathrm{C}_{22} \mathrm{H}_{27} \mathrm{~N}_{2} \mathrm{O}_{3}{ }^{+}[\mathrm{M}+\mathrm{H}]^{+}$367.2016, found 367.2017.

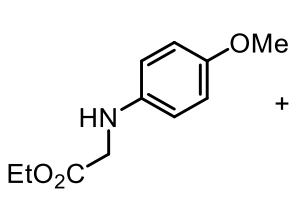

$1 \mathrm{k}$

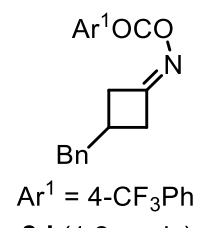

2d (1.2 equiv)

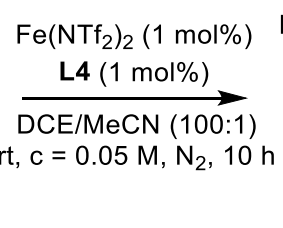

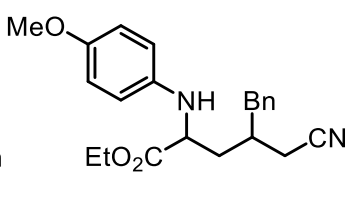

3kd, $87 \%, d r=1.2$

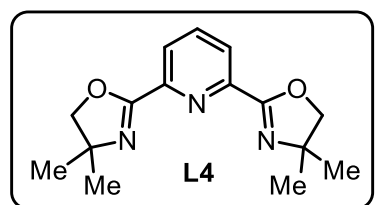


Ethyl 4-benzyl-5-cyano-2-((4-methoxyphenyl)amino)pentanoate (3kd): Under condition B, compound 3kd was prepared by reacting $\mathbf{1 k}$ with $\mathbf{2 d}$, and the product was isolated through a silica gel flash column (PE: EtOAc, from 10:1 to 5:1) as yellowish oil (64 mg, 81\% yield, $d r=1.2) .{ }^{1} \mathrm{H}$ NMR (400 MHz, Chloroform- $d$ ) (mixture of 2 diastereomers, 1.2:1) $\delta 7.38-7.26(\mathrm{~m}, 2 \mathrm{H}$, isomer $1+2 \mathrm{H}$, isomer 2), 7.29 $-7.20(\mathrm{~m}, 1 \mathrm{H}$, isomer $1+1 \mathrm{H}$, isomer 2$), 7.23-7.12(\mathrm{~m}, 2 \mathrm{H}$, isomer $1+2 \mathrm{H}$, isomer 2$), 6.82-6.72(\mathrm{~m}$, $2 \mathrm{H}$, isomer $1+2 \mathrm{H}$, isomer 2$), 6.68-6.55(\mathrm{~m}, 2 \mathrm{H}$, isomer $1+2 \mathrm{H}$, isomer 2$), 4.21-4.08(\mathrm{~m}, 2 \mathrm{H}$, isomer $1+2 \mathrm{H}$, isomer 2), $4.07-3.99(\mathrm{~m}, 1 \mathrm{H}$, isomer $1+1 \mathrm{H}$, isomer 2$), 3.82-3.74(\mathrm{br}, 1 \mathrm{H}$, isomer $1+1 \mathrm{H}$, isomer 2), $3.74(\mathrm{~s}, 3 \mathrm{H}$, isomer 1), $3.73(\mathrm{~s}, 3 \mathrm{H}$, isomer 2), $3.00-2.80(\mathrm{~m}, 1 \mathrm{H}$, isomer $1+1 \mathrm{H}$, isomer 2$)$, $2.70(\mathrm{dd}, J=13.7,8.2 \mathrm{~Hz}, 1 \mathrm{H}$, isomer 2), $2.63(\mathrm{dd}, J=13.8,8.8 \mathrm{~Hz}, 1 \mathrm{H}$, isomer 1), $2.50-2.40(\mathrm{~m}, 1 \mathrm{H}$, isomer 1), $2.39-2.24(\mathrm{~m}, 2 \mathrm{H}$, isomer $1+3 \mathrm{H}$, isomer 2), $2.03-1.93(\mathrm{~m}, 1 \mathrm{H}$, isomer $1+1 \mathrm{H}$, isomer 2$)$, $1.93-1.83(\mathrm{~m}, 1 \mathrm{H}$, isomer $1+1 \mathrm{H}$, isomer 2$), 1.25-1.19(\mathrm{~m}, 3 \mathrm{H}$, isomer $1+3 \mathrm{H}$, isomer 2$) ;{ }^{13} \mathrm{C}\left\{{ }^{1} \mathrm{H}\right\} \mathrm{NMR}$ $(101 \mathrm{MHz}$, Chloroform- $d$ ) $\delta 174.0$ (isomer $1+$ isomer 2), 153.2 (isomer $1+$ isomer 2), 140.7, 140.5, 138.3, 138.1, 129.1 (isomer $1+$ isomer 2), 128.8 (isomer $1+$ isomer 2), 126.8 (isomer $1+$ isomer 2), 118.2, 118.0, 115.7, 115.5, 114.93, 114.91, 61.37, 61.34, 56.5, 55.8, 55.7 (isomer $1+$ isomer 2), 40.0, 39.6, 36.9, 36.3, 34.5, 34.0, 21.5, 21.0, 14.2, 14.2; IR $v_{\max }$ (neat) $/ \mathrm{cm}^{-1}: 3358,2932,2831,2245,1731,1512,1454$, 1237, 1184, 1134, 1034; HRMS (ESI, m/z): calcd for $\mathrm{C}_{22} \mathrm{H}_{27} \mathrm{~N}_{2} \mathrm{O}_{3}{ }^{+}[\mathrm{M}+\mathrm{H}]^{+}$367.2016, found 367.2018.
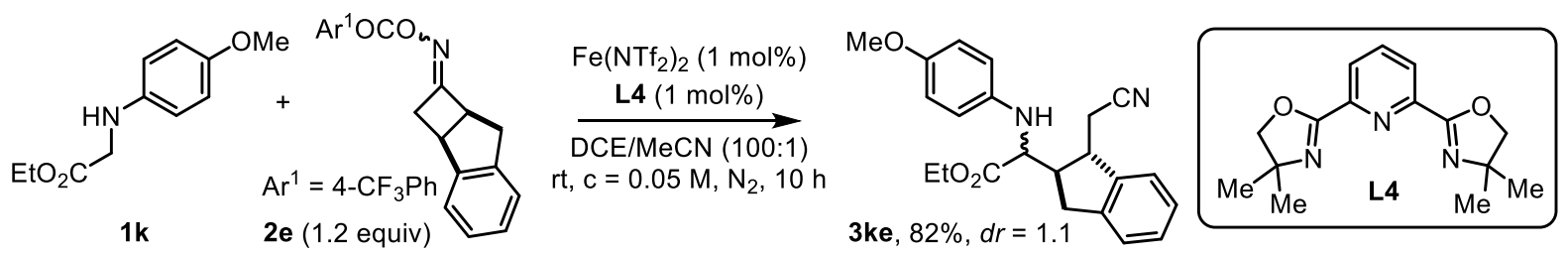

Ethyl 2-(1-(cyanomethyl)-2,3-dihydro-1H-inden-2-yl)-2-((4-methoxyphenyl)amino)acetate

(3ke):

Under condition B, compound 3ke was prepared by reacting $\mathbf{1 k}$ with $\mathbf{2 e}$, and the product was isolated through a silica gel flash column (PE: EtOAc, from 10:1 to 5:1) as yellow oil $(60 \mathrm{mg}, 82 \%$ yield, $d r=$ 1.1). ${ }^{1} \mathrm{H}$ NMR (400 MHz, Chloroform- $d$ ) $\delta 7.35-7.23(\mathrm{~m}, 4 \mathrm{H}$, isomer $1+4 \mathrm{H}$, isomer 2), $6.84-6.74(\mathrm{~m}$, $2 \mathrm{H}$, isomer $1+2 \mathrm{H}$, isomer 2$), 6.72-6.61(\mathrm{~m}, 2 \mathrm{H}$, isomer $1+2 \mathrm{H}$, isomer 2$), 4.23-4.05(\mathrm{~m}, 3 \mathrm{H}$, isomer $1+2 \mathrm{H}$, isomer 2), $4.02(\mathrm{~d}, J=8.2 \mathrm{~Hz}, 1 \mathrm{H}$, isomer 2$), 3.76(\mathrm{~s}, 3 \mathrm{H}$, isomer $1+3 \mathrm{H}$, isomer 2$), 3.71-3.55$ $(\mathrm{m}, 1 \mathrm{H}$, isomer $1+1 \mathrm{H}$, isomer 2$), 3.19(\mathrm{dd}, J=16.5,8.5 \mathrm{~Hz}, 1 \mathrm{H}$, isomer $1+1 \mathrm{H}$, isomer 2$), 3.10(\mathrm{dd}, J=$ 16.7, 6.1 Hz, 1H, isomer 2), $2.96(\mathrm{dd}, J=16.4,5.8 \mathrm{~Hz}, 1 \mathrm{H}$, isomer 1$), 2.86-2.68(\mathrm{~m}, 3 \mathrm{H}$, isomer $1+3 \mathrm{H}$, isomer 2), $1.26-1.19(\mathrm{~m}, 3 \mathrm{H}$, isomer $1+3 \mathrm{H}$, isomer 2$) ;{ }^{13} \mathrm{C}\left\{{ }^{1} \mathrm{H}\right\} \mathrm{NMR}(101 \mathrm{MHz}$, Chloroform- $d) \delta 173.3$, 173.1, 153.2, 153.1, 142.0, 142.0, 141.47, 141.45, 140.7, 140.5, 128.11, 128.09, 127.2 (isomer $1+$ isomer 2), 125.02, 124.97, 123.9, 123.8, 118.6, 118.4, 115.8, 115.6, 114.91, 114.89, 61.6, 61.5, 61.3, 60.0, 55.7 (isomer 1 + isomer 2), 47.4, 47.1, 44.8, 43.2, 34.1, 33.6, 23.3, 22.9, 14.22, 14.20; IR $v_{\max }(\mathrm{neat}) / \mathrm{cm}^{-1}$ : 3359, 2934, 2833, 2246, 1725, 1510, 1460, 1235, 1180, 1032; HRMS (ESI, m/z): calcd for $\mathrm{C}_{22} \mathrm{H}_{25} \mathrm{~N}_{2} \mathrm{O}_{3}{ }^{+}$ $[\mathrm{M}+\mathrm{H}]^{+} 365.1860$, found 365.1864 . 

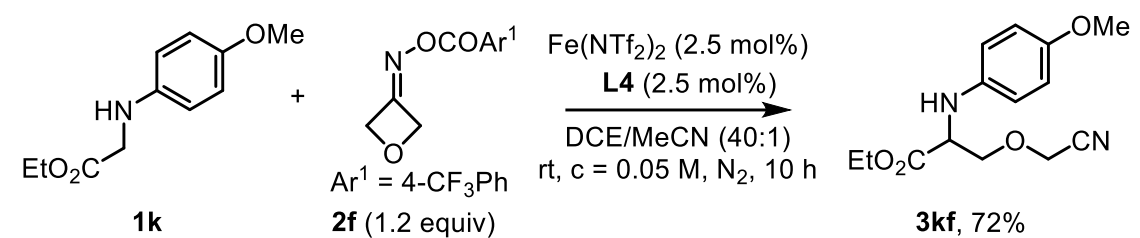

3kf, $72 \%$

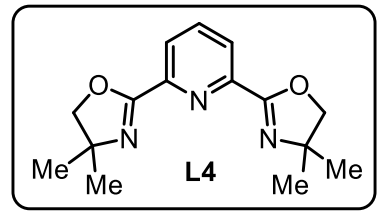

Ethyl O-(cyanomethyl)-N-(4-methoxyphenyl)serinate (3kf): Under condition B, compound 3kf was prepared by reacting $\mathbf{1 k}$ with $\mathbf{2 f}$ in the presence of $2.5 \mathrm{~mol} \%$ catalysts (the solvent ratio changed accordingly), and the product was isolated through a silica gel flash column (PE: EtOAc, from 10:1 to 5:1) as yellow oil (40 mg, 72\% yield). ${ }^{1} \mathrm{H}$ NMR (400 MHz, Chloroform- $d$ ) $\delta 6.83-6.73(\mathrm{~m}, 2 \mathrm{H}), 6.67-$ $6.58(\mathrm{~m}, 2 \mathrm{H}), 4.34(\mathrm{~d}, J=16.2 \mathrm{~Hz}, 1 \mathrm{H}), 4.25(\mathrm{~d}, J=16.2 \mathrm{~Hz}, 1 \mathrm{H}), 4.27-4.16(\mathrm{~m}, 4 \mathrm{H}), 4.02(\mathrm{dd}, J=9.0$, $3.8 \mathrm{~Hz}, 1 \mathrm{H}), 3.91(\mathrm{dd}, J=9.1,3.7 \mathrm{~Hz}, 1 \mathrm{H}), 3.74(\mathrm{~s}, 3 \mathrm{H}), 1.27(\mathrm{t}, J=7.1 \mathrm{~Hz}, 3 \mathrm{H}) ;{ }^{13} \mathrm{C}\left\{{ }^{1} \mathrm{H}\right\} \mathrm{NMR}(101$ MHz, Chloroform- $d$ ) $\delta 171.1,153.2,140.1,115.5,115.4,114.9,71.7,61.8,57.9,56.8,55.7,14.2$; IR $v_{\max }$ (neat) $/ \mathrm{cm}^{-1}: 3369,2935,1514,1464,1236,1199,1155,1108,1035 ;$ HRMS (ESI, m/z): calcd for $\mathrm{C}_{14} \mathrm{H}_{19} \mathrm{~N}_{2} \mathrm{O}_{4}^{+}[\mathrm{M}+\mathrm{H}]^{+} 279.1339$, found 279.1342 .

\section{Control Experiments and Mechanistic Studies}

\section{a) Probing for active radical species in the reaction}

\section{i) Detection of homo-coupling products with increased catalyst loading}
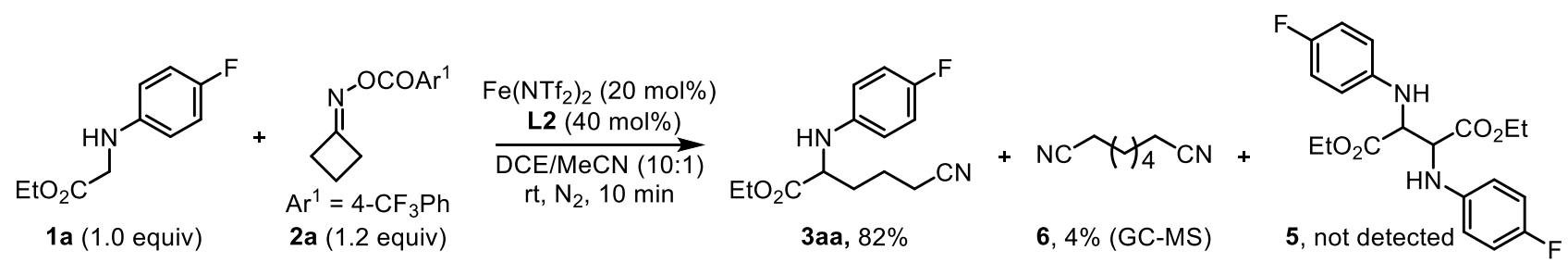

In a $\mathrm{N}_{2}$ atmosphere glovebox, $\mathrm{Fe}\left(\mathrm{NTf}_{2}\right)_{2}(24.6 \mathrm{mg}, 0.04 \mathrm{mmol})$, and $\mathbf{L 2}(14.1 \mathrm{mg}, 0.08 \mathrm{mmol})$ were weighed into a 2-dram vial charged with a stir bar. The vial was sealed with a septum and taken out of the glovebox. Then, freshly distilled dry 1,2-dichloroethane $(0.8 \mathrm{~mL})$ and dry $\mathrm{MeCN}(0.2 \mathrm{~mL})$ was added into the vial. The mixture was stirred at room temperature to afford a brown solution. Outside the glovebox, to another flame-dried sealable 2-dram vial charged with a stir bar were added glycinate 1a (39.4 $\mathrm{mg}, 0.2 \mathrm{mmol})$ and cyclobutanone oxime ester $\mathbf{2 a}(62 \mathrm{mg}, 0.24 \mathrm{mmol})$. After the vial was evacuated and backfilled with $\mathrm{N}_{2}$ three times, anhydrous 1,2-dichloroethane $(1.0 \mathrm{~mL})$ was added, followed by the addition of the above catalyst solution. The resulting mixture was stirred at room temperature ( 23 to $26{ }^{\circ} \mathrm{C}$ ) for $10 \mathrm{~min}$ and quenched with saturated $\mathrm{NaHCO}_{3}$ aqueous solution $(2 \mathrm{~mL})$. After the organic layer was separated, the aqueous phase was extracted with $\mathrm{CH}_{2} \mathrm{Cl}_{2}(1 \mathrm{~mL} \times 3)$. The combined organic layers were dried over anhydrous $\mathrm{Na}_{2} \mathrm{SO}_{4}$, filtered, and then concentrated in vacuo to afford the crude product, which was submitted for NMR and GC-MS analysis successively. A homocoupling product $\mathbf{6}$ between two butyronitrile-4-yl radicals was identified in both GC-MS and ${ }^{1} \mathrm{H}$ NMR by using a commercially purchased 
product as the reference (CAS: 629-40-3). On the other hand, homocoupling product 5 was not detected by ${ }^{19}$ F NMR (see Figure $\mathbf{S 2}$ for stacked spectra).

1) Glycine ester homo-coupling product

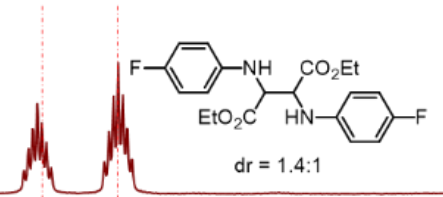

2) Cross-coupling product

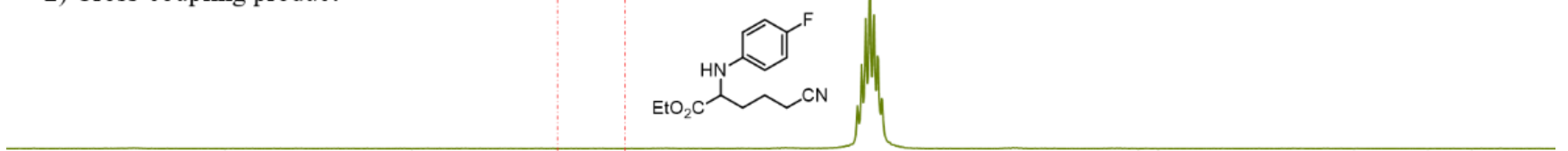

3) Crude ${ }^{19} \mathrm{~F}$ NMR of $\operatorname{Ir}(\text { ppy })_{3}$ catalyzed reaction (with $\mathrm{DABCO}$ )

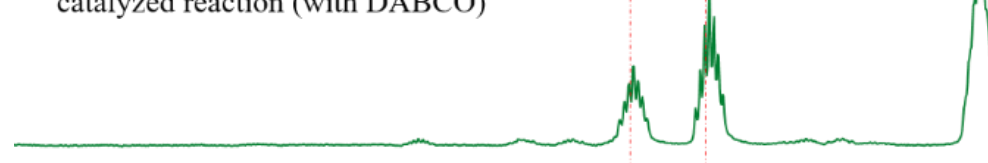

4) Crude ${ }^{19} \mathrm{~F}$ NMR of $\operatorname{Ir}(\text { ppy })_{3}$ catalyzed reaction (without DABCO)

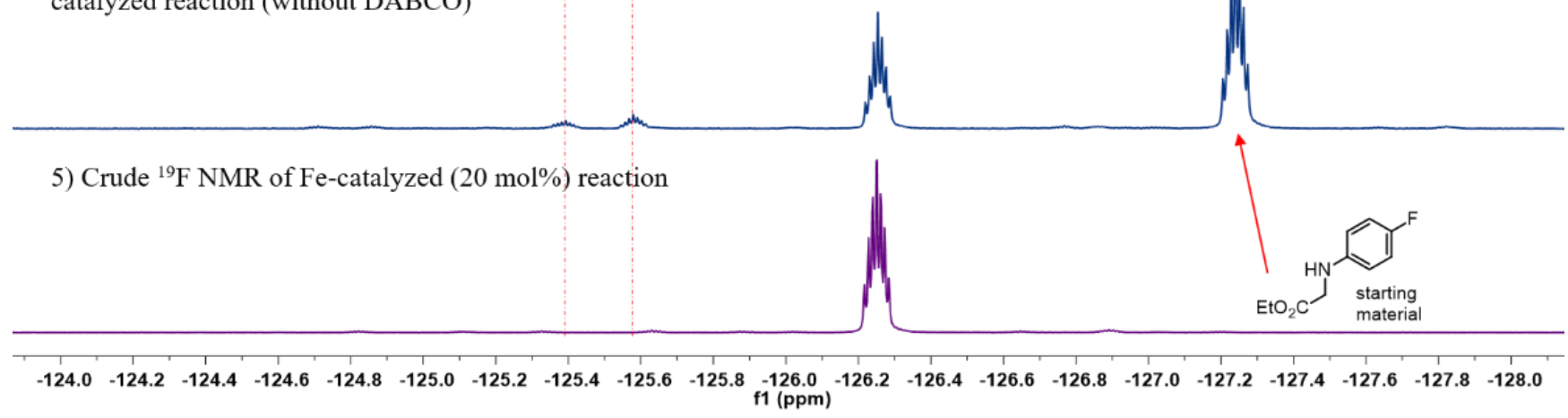

Figure S2. The stacked ${ }^{19}$ F NMR of crude products obtained with different catalysts

\section{ii) Radical trapping experiment}<smiles>CCOC(=O)CNc1ccc(F)cc1</smiles>

$1 \mathbf{a}(1.0$ equiv)<smiles>CC1(C)CCCC(C)(C)N1OCOCCOCCOCCO</smiles>

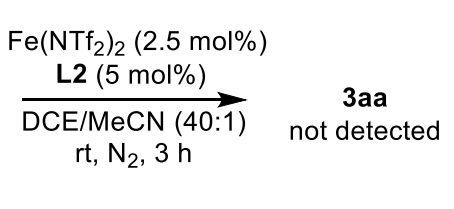<smiles>CCCCON1C2CCCCC2CCC1(C)C</smiles>

The reaction between $\mathbf{1 a}$ and $\mathbf{2 a}$ was set up according to the procedures for condition A, but in the presence of TEMPO ((2,2,6,6-tetramethylpiperidin-1-yl)oxyl) $(31.3 \mathrm{mg}, 0.2 \mathrm{mmol})$. The reaction was quenched with saturated $\mathrm{NaHCO}_{3}$ aqueous solution after $3 \mathrm{~h}$ and worked-up following the general procedure. The desired product 3aa was not detected by TLC, with large amount of 1a, 2a and TEMPO recovered. The crude product was then submitted for HRMS analysis to identify the cyanoalkyl radical trapping product S5 (Figure S3). HRMS (ESI, m/z): calcd for $\mathrm{C}_{13} \mathrm{H}_{25} \mathrm{~N}_{2} \mathrm{O}^{+}[\mathrm{M}+\mathrm{H}]^{+} 225.1961$, found 225.1966. 


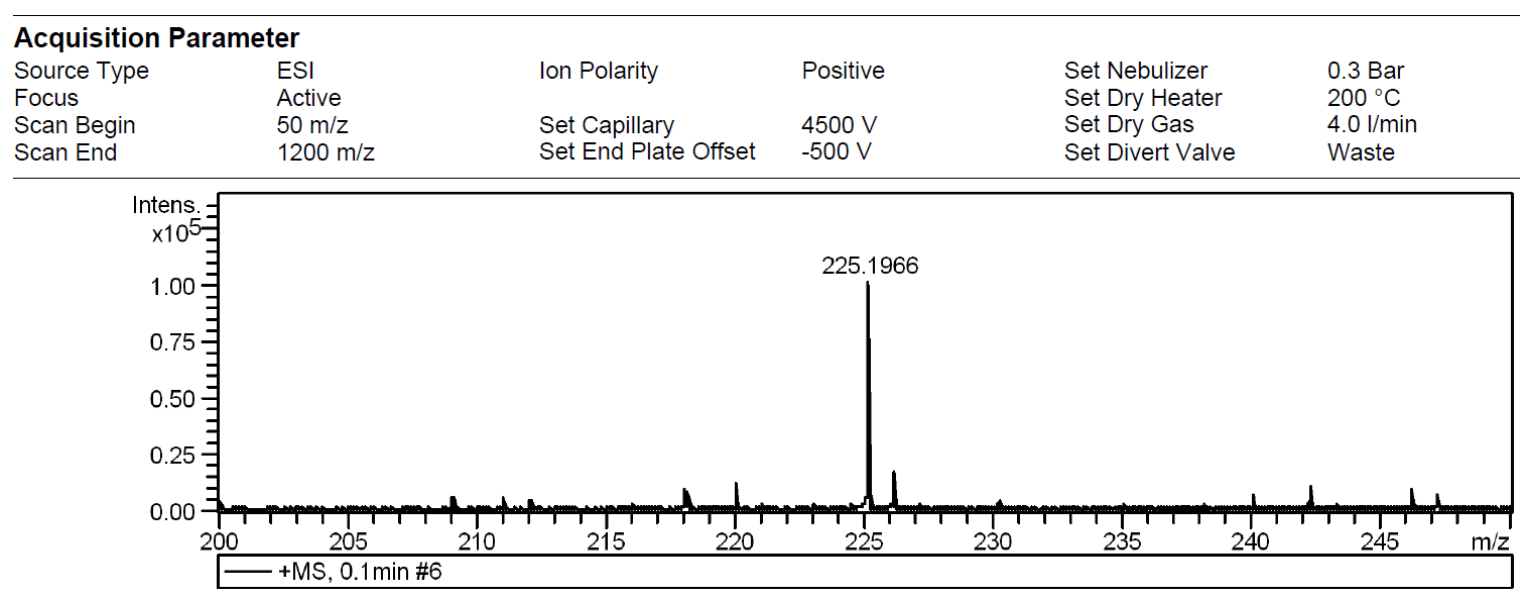

Figure S3. The HRMS result for radical trapping product

b) The participation of an exogenous imine in the coupling reaction

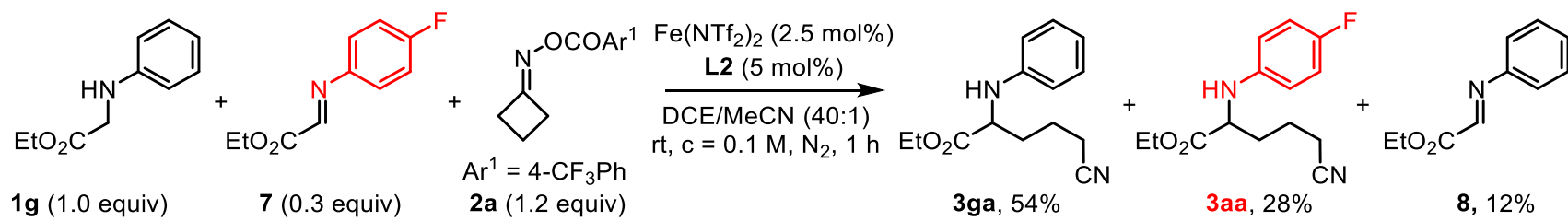

The reaction between $\mathbf{1 g}$ and $\mathbf{2 a}$ was set up according to the procedures for condition A, but a $N$-(4fluorophenyl) imine $(12 \mathrm{mg}, 0.06 \mathrm{mmol})$ was added to the reaction together with $\mathbf{1 g}$. The reaction was quenched with saturated $\mathrm{NaHCO}_{3}$ aqueous solution after $1 \mathrm{~h}$, worked-up following the general procedure, and the crude product was submitted for ${ }^{1} \mathrm{H}$ and ${ }^{19} \mathrm{~F}$ NMR analysis. Besides the anticipated coupling product 3ga, fluorine-labeled product 3aa was also obtained in $28 \%$ yield, together with the generation of $N$-phenyl imine 8 (Figure S4 \& S5).

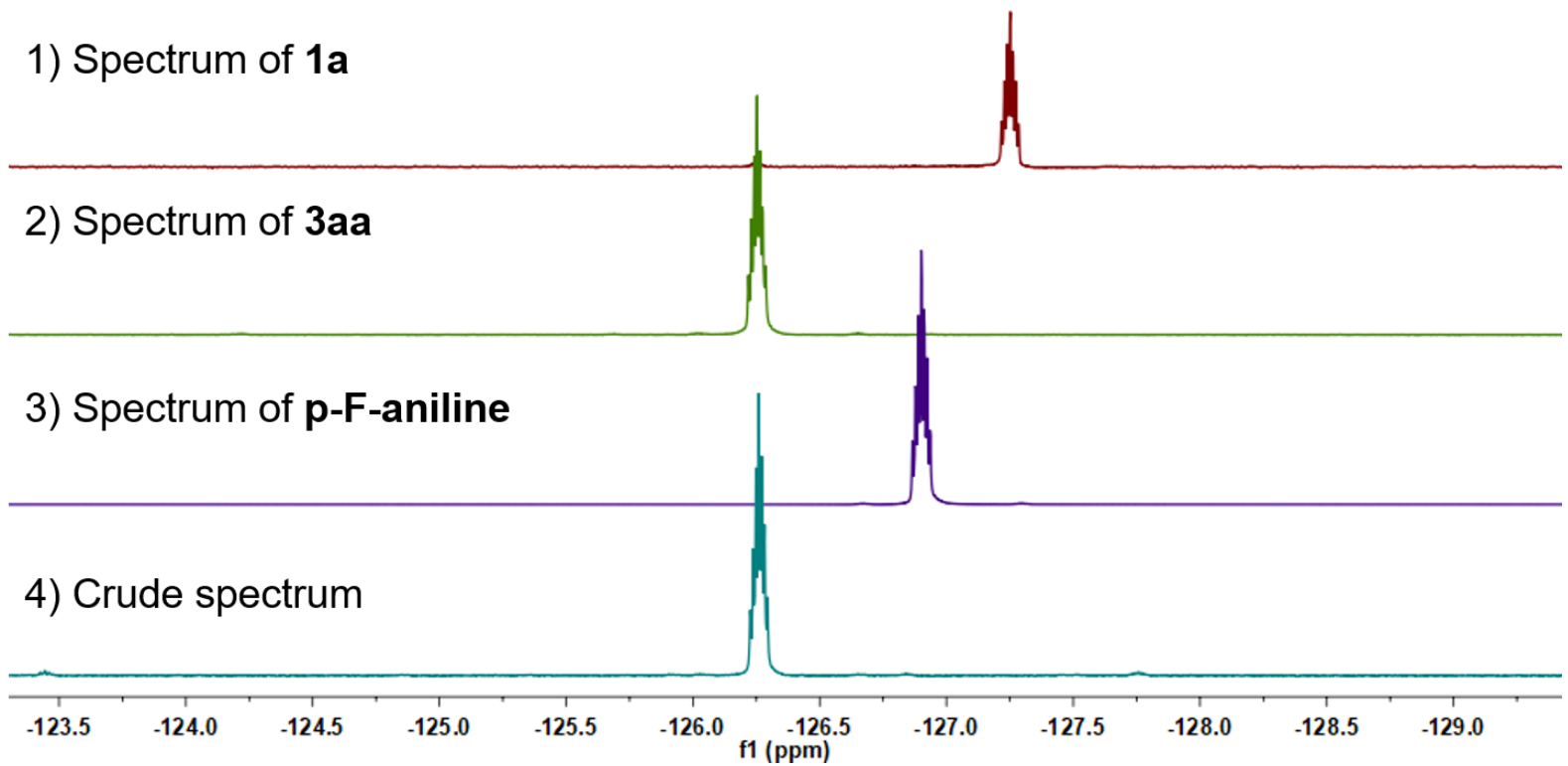

Figure S4. Stacked ${ }^{19}$ F NMR to confirm the generation of 3aa 
1) Spectrum of $3 g a$

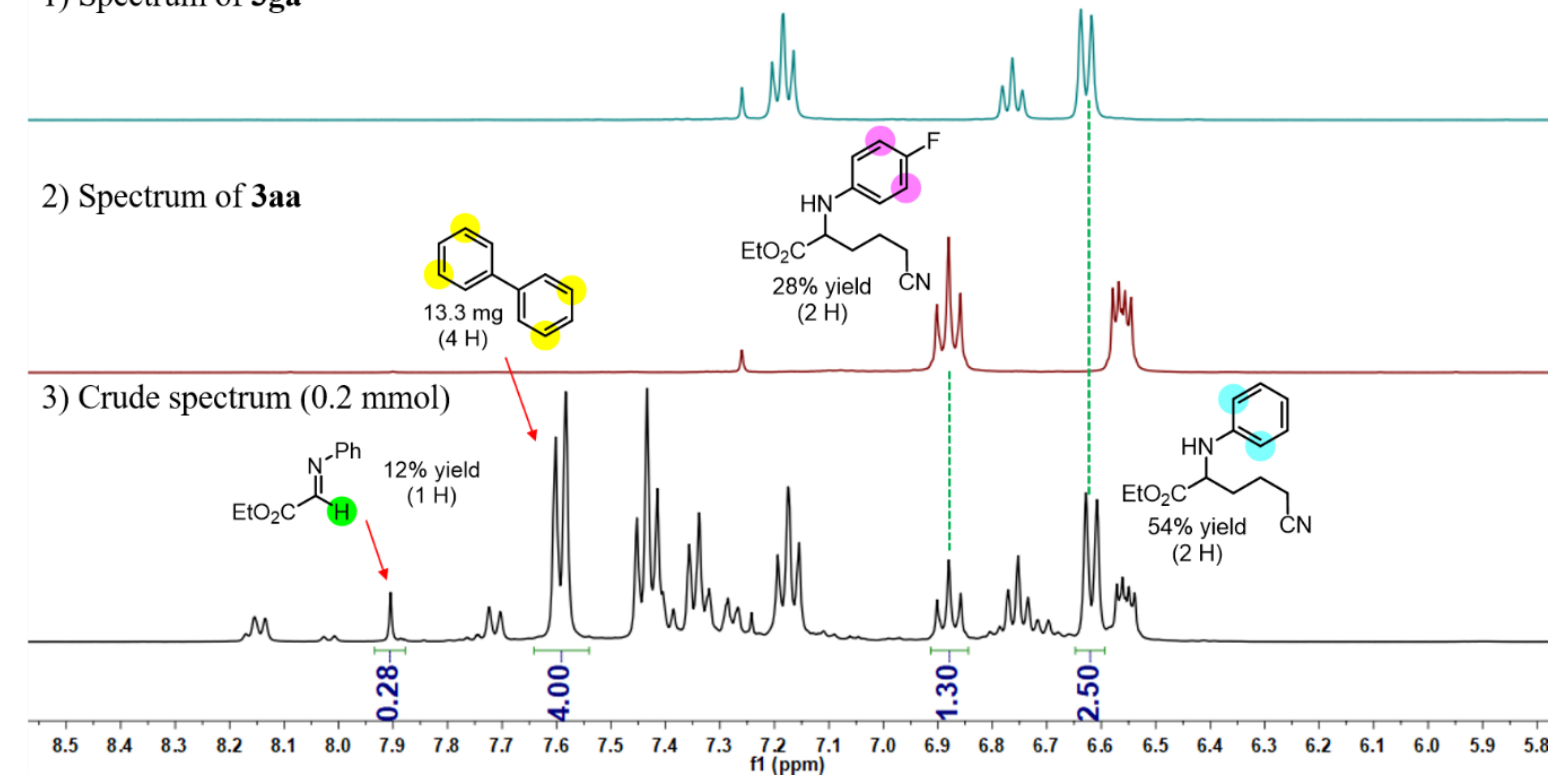

Fugure S5. Crude ${ }^{1} \mathrm{H}$ NMR analysis to determine the products distribution

c) The integration of 4-F-aniline into the product through an imine-aniline exchange

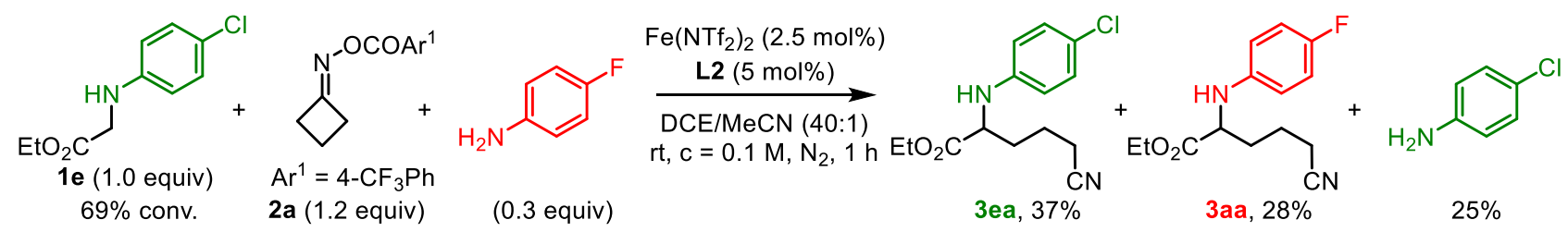

The reaction was set up according to the procedures for condition $\mathrm{A}$, but a freshly distilled $p$-fluoroaniline $(6 \mu \mathrm{L}, 0.06 \mathrm{mmol})$ was added to the reaction before the addition of the catalyst. The reaction was quenched with saturated $\mathrm{NaHCO}_{3}$ aqueous solution after $1 \mathrm{~h}$, worked-up following the general procedure, and the crude product was submitted for ${ }^{1} \mathrm{H}$ and ${ }^{19} \mathrm{~F}$ NMR analysis. 4-F-aniline was consumed and only one new F-containing species (3aa) was observed on ${ }^{19} \mathrm{~F}$ NMR (Figure S6).

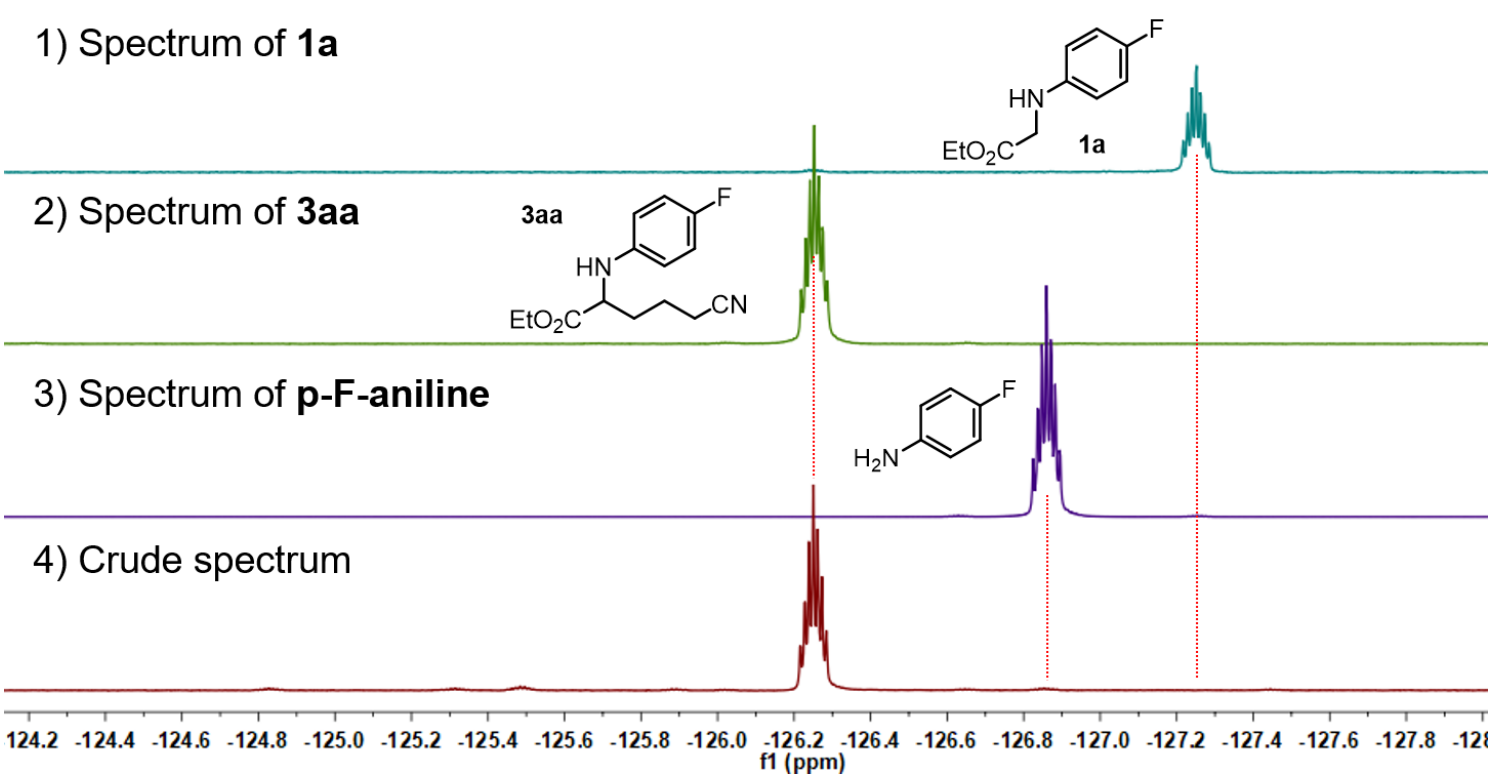

Fugure S6. Stacked ${ }^{19}$ F NMR spectrum to determine the conversion of 4 -F aniline 
The quantitative analysis of each component was carried out by ${ }^{1} \mathrm{H}$ NMR with trimethoxybenzene as an internal standard. The conversion of 1e and the yield of 3aa was determined directly by the integration of their characteristic peaks, repectively. While the yield of 3ea was then determined by deducing 3aa from the combined yield of cyanoalkylation products. Then, the yield of 4-Cl-aniline was calculated similarly as illustrated in Figure S7.

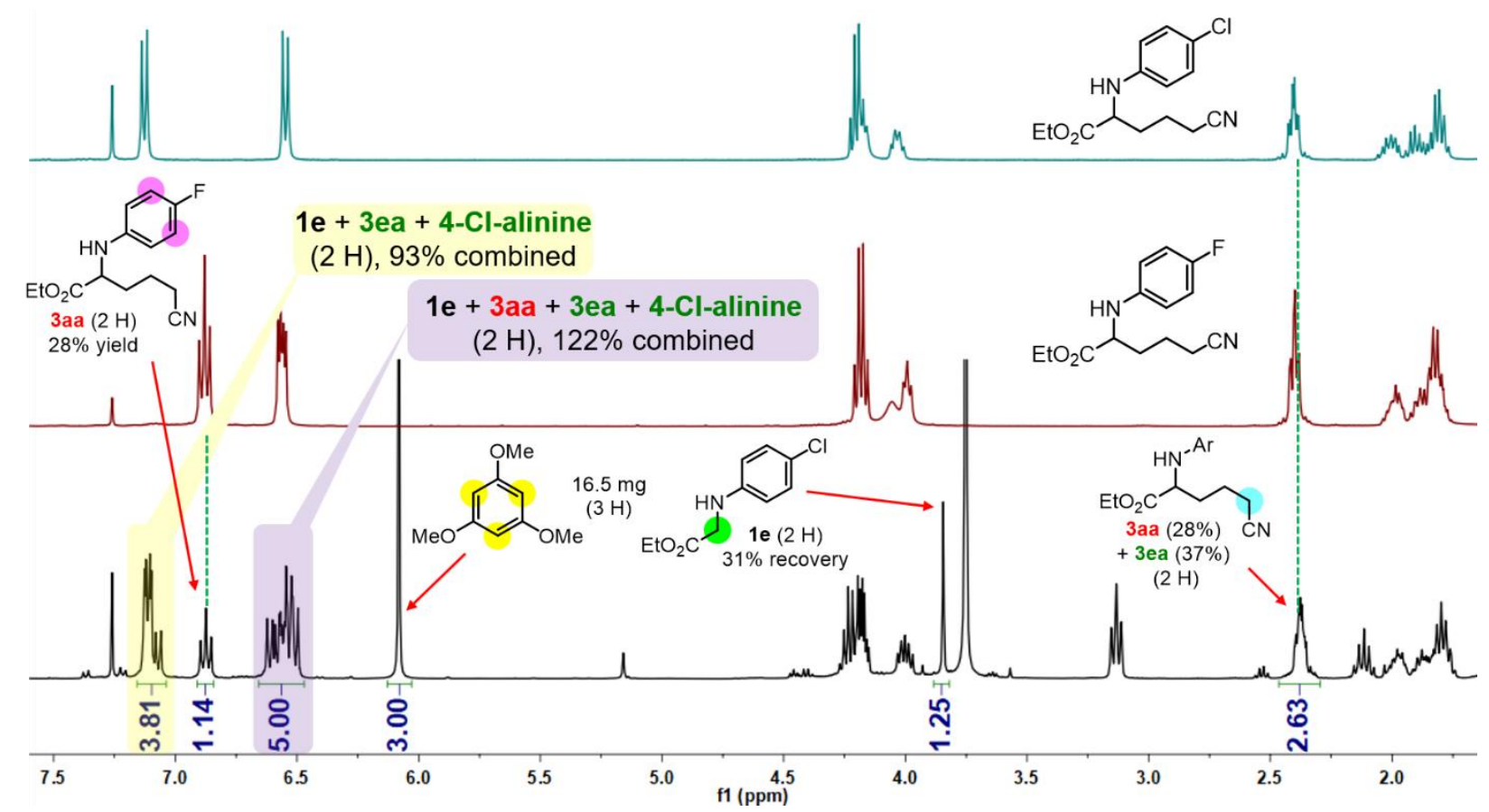

Figure S7. Crude NMR analysis to determine the products distribution

In order to figure out how this occurs, a series of control experiments were performed. First, when 3ea was treated with $p$-fluoroaniline under the reaction conditions, 3aa was NOT detected by ${ }^{19} \mathrm{~F} N M R$.

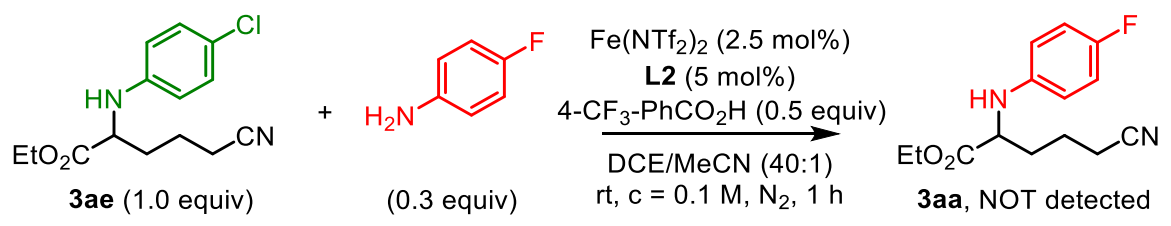

Then, treating glycinate 1e with $p$-fluoroaniline under the reaction conditions did NOT lead to any substitution product 1a either.

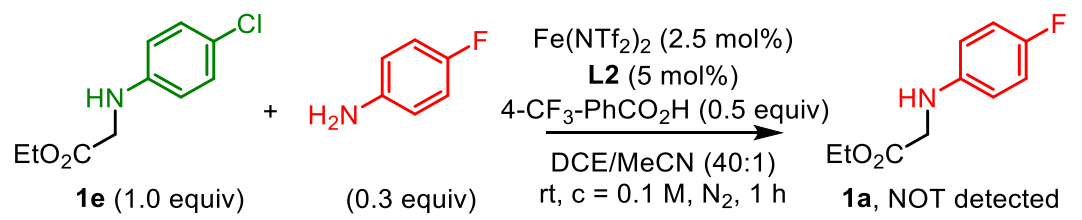

Next, the chorine-substituted imine underwent a rapid exchange with $p$-fluoroaniline under the reaction conditions, affording a fluorine-substituted imine 7. The latter is known to react with a cyanoalkyl radical to afford product 3aa. 


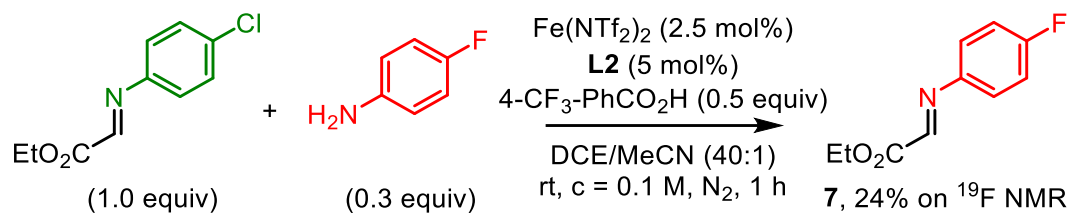

\section{d) The cyclic voltammetry studies of substrates and catalysts}

The reduction/oxidation potentials were determined by cyclic voltammograms of each compound on a potentiostat. Conditions: glassy carbon as the working electrode, Pt plate as the counter electrode, $\mathrm{Ag} / \mathrm{AgNO}_{3}(0.1 \mathrm{M}$ in $\mathrm{MeCN})$ as the reference electrode, $\mathrm{Bu}_{4} \mathrm{NBF}_{4}$ in $\mathrm{MeCN}(0.1 \mathrm{M})$ was used as the supporting electrolyte, scan rate $=50 \mathrm{mV} \cdot \mathrm{s}^{-1}$

First, the redox potentials of the substrates were measured in $\mathrm{MeCN}(0.025 \mathrm{M})$ with the supporting electrolyte respectively (Table S3). Irreversible oxidation of 1a was recorded with a half-wave potential at $0.65 \mathrm{~V}$ vs $\mathrm{Ag} / \mathrm{AgNO}_{3}(1.01 \mathrm{~V}$ vs $\mathrm{SCE})$. On the other hand, an irreversible reduction of 2a was observed with a half-wave potential at $-1.38 \mathrm{~V}$ vs $\mathrm{Ag} / \mathrm{AgNO}_{3}(-1.02 \mathrm{~V}$ vs SCE).

Table S3. The cyclic voltammograms of substrates

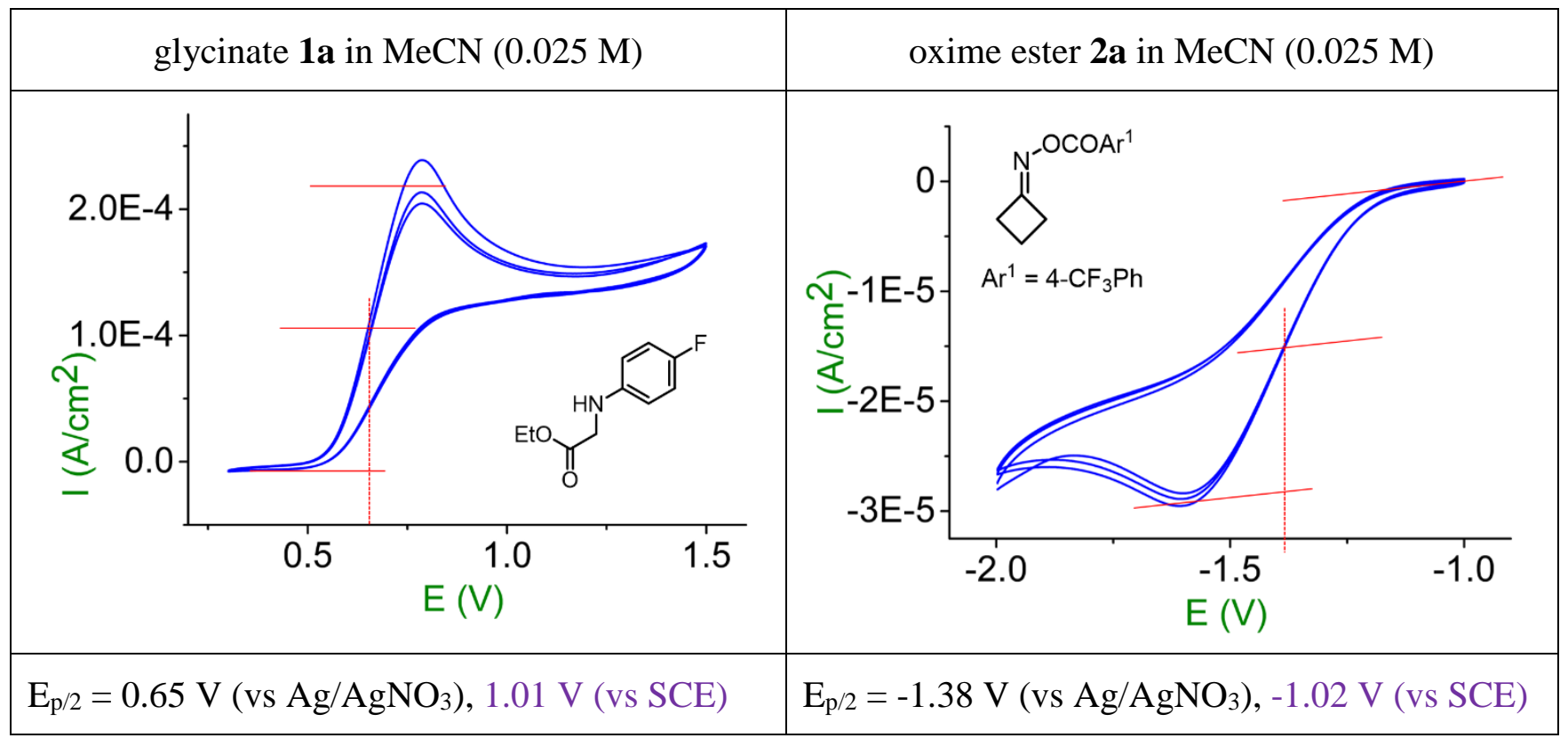

Next, representative $\mathrm{Fe}\left(\mathrm{NTf}_{2}\right)_{2}$-ligand complexes are also evaluated with cyclic voltammetry (see data in Table S4). Fe( $\left.\mathrm{NTf}_{2}\right)_{2}(12.3 \mathrm{mg}, 0.02 \mathrm{mmol})$ and a corresponding ligand (0.04 mmol) were weighed into a 2-dram-vial and then dissolved in the electrolyte solution $(4 \mathrm{~mL})$, respectively. The clear solutions were then submitted for voltammograms scan. No distinct oxidation-reduction peaks were observed for $\mathrm{Fe}\left(\mathrm{NTf}_{2}\right)_{2}$ alone, while iron-L1 complex is redox-active. Unexpectedly, as a uniquely efficient catalyst, the iron-L2 complex undergoes irreversible oxidation at an even higher potential. A similar irreversible redox pattern was observed for iron-L4, which was also catalytically active at room temperature. 
Table S4. The cyclic voltammograms of representative catalysts

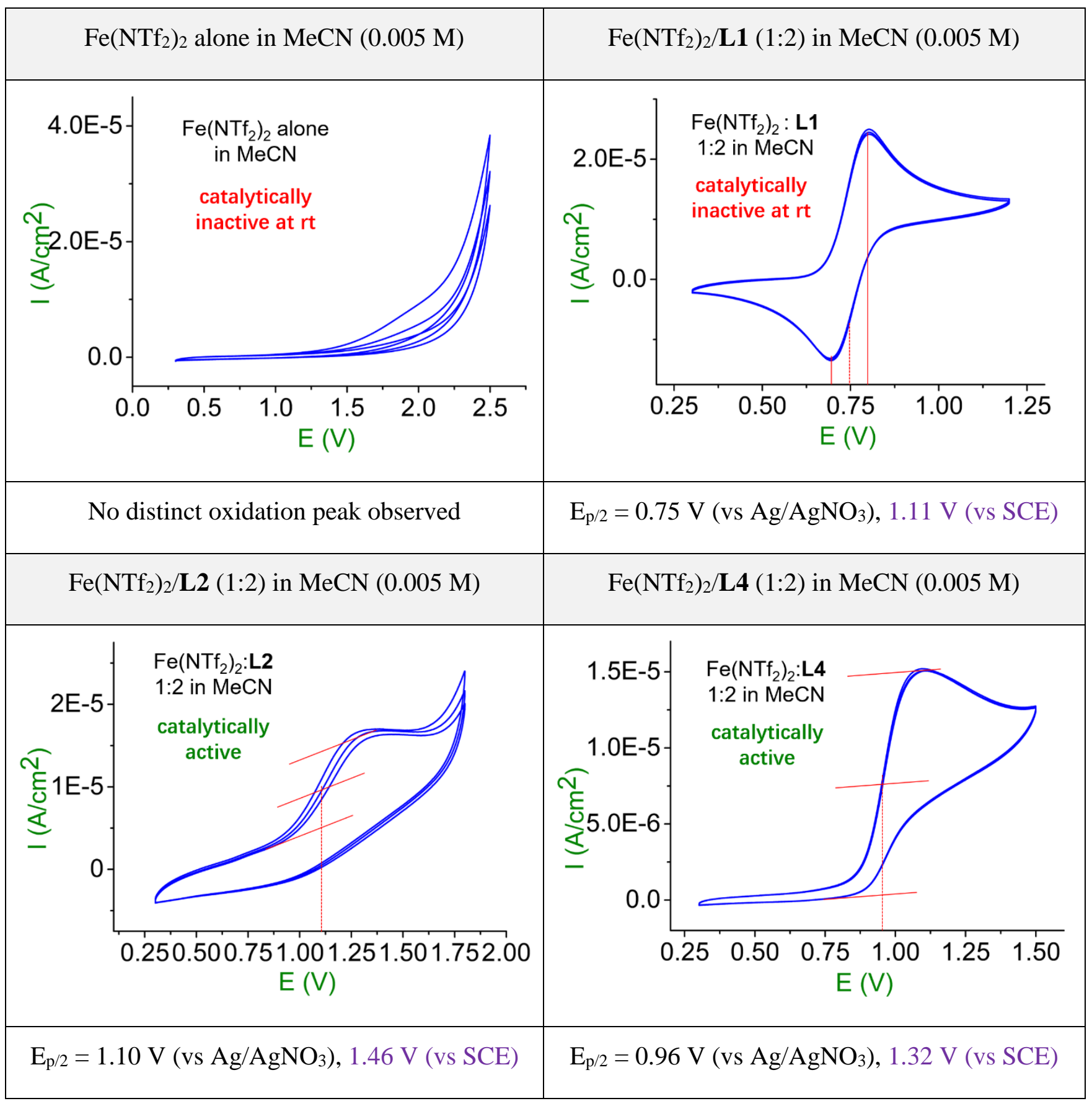

We managed to mix the $\mathrm{Fe}\left(\mathrm{NTf}_{2}\right)_{2}-\mathbf{L} \mathbf{2}$ complex with $\mathbf{1 a}$ (molar ratio = 1:10) to determine whether a more reductive species was formed. Nevertheless, only the oxidation peak of 1a was observed. These results of cyclic voltammetry studies suggested that the mechanism of this iron-catalyzed reaction is more complicated than an outer-sphere single-electron transfer redox system.

e) The chelation-acceleration effect in the decomposition of oxime ester 


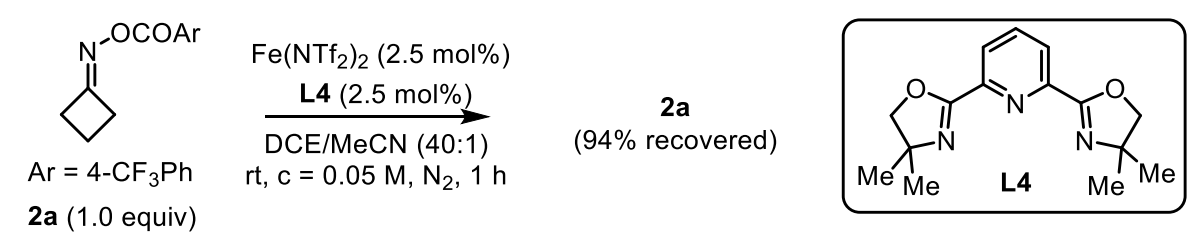

Cyclobutanone oxime ester 2a was treated with $\mathrm{Fe}\left(\mathrm{NTf}_{2}\right)_{2} / \mathbf{L} \mathbf{4}(2.5 \mathrm{~mol} \%)$ under condition B for $1 \mathrm{~h} . \mathbf{2 a}$ was recovered in 94\% (NMR yield) after the routine quenching and work-up procedures.

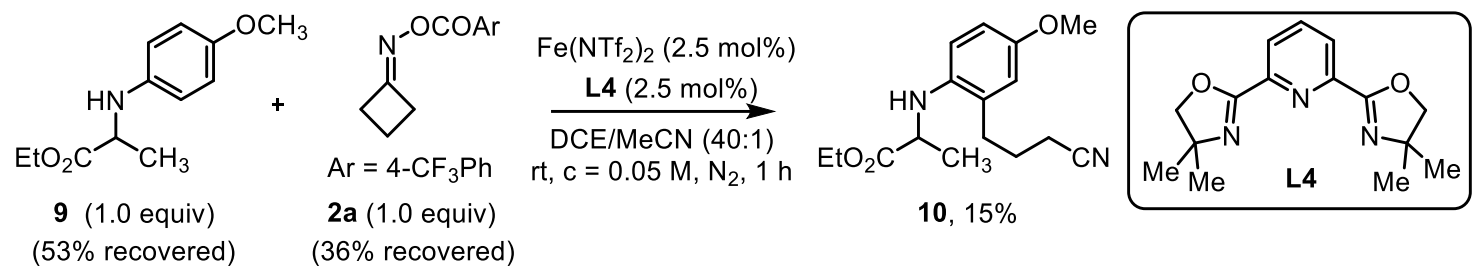

Ethyl (2-(3-cyanopropyl)-4-methoxyphenyl)alaninate (10): When a N-PMP protected alanine was employed as a substrate under condition $\mathrm{B}$, the desired $\mathrm{C}_{(\mathrm{sp} 3)}-\mathrm{H}$ coupling product was not observed. Instead, an aryl alkylation product was generated in the early stage of the reaction, along with several indistinct decomposition products after $1 \mathrm{~h}$. Compound $\mathbf{1 0}$ was isolated through a silica gel flash column (PE: EtOAc from 20:1 to 10:1) as yellowish oil (9 mg, 15\%). ${ }^{1} \mathrm{H}$ NMR (400 MHz, Chloroform- $d$ ) $\delta 6.72$ $-6.65(\mathrm{~m}, 2 \mathrm{H}), 6.53(\mathrm{~d}, J=8.5 \mathrm{~Hz}, 1 \mathrm{H}), 4.17$ (q, $J=7.1 \mathrm{~Hz}, 2 \mathrm{H}), 4.08$ (q, $J=6.9 \mathrm{~Hz}, 1 \mathrm{H}), 3.81$ (brs, 1H), $3.74(\mathrm{~s}, 3 \mathrm{H}), 2.74-2.64(\mathrm{~m}, 2 \mathrm{H}), 2.43-2.35(\mathrm{~m}, 2 \mathrm{H}), 2.01$ (p, J=7.2 Hz, 2H), 1.49 (d, J=6.9 Hz, 3H), $1.24(\mathrm{t}, J=7.1 \mathrm{~Hz}, 3 \mathrm{H}) ;{ }^{13} \mathrm{C}\left\{{ }^{1} \mathrm{H}\right\} \mathrm{NMR}(101 \mathrm{MHz}$, Chloroform- $d) \delta 175.0,152.5,138.5,126.4,119.7$, 116.4, 113.2, 112.4, 61.1, 55.6, 52.9, 30.0, 24.6, 19.2, 16.7, 14.2; IR $v_{\max }$ (neat)/cm ${ }^{-1}: 3384,2980,2936$, 2245, 1729, 1510, 1452, 1301, 1228, 1157, 1052, 803; HRMS (ESI, m/z): calcd for $\mathrm{C}_{16} \mathrm{H}_{23} \mathrm{~N}_{2} \mathrm{O}_{3}{ }^{+}[\mathrm{M}+$ $\mathrm{H}]^{+}$291.1703, found 291.1698.

Although the coupling reaction of alanine ester 9 was not successful, we proposed to use it as a mechanistic probe to see if the $\alpha$-amino carbonyl moiety played a role in activating Fe catalyst and thereby, facilitating the decomposition of oxime esters. If the ester carbonyl only acted as an electron-withdrawing group in the above reaction, then a cyano analog $\mathbf{1 1}$ would do the same job because its aryl group is basically identical in terms of electron properties. On the other hand, if the ester carbonyl played a role in activating the catalyst through chelation, different reactivity would be anticipated with $\mathbf{1 1}$.

As a result, $N$-PMP-2-aminopropanenitrile 11 was prepared and employed in a control reaction.
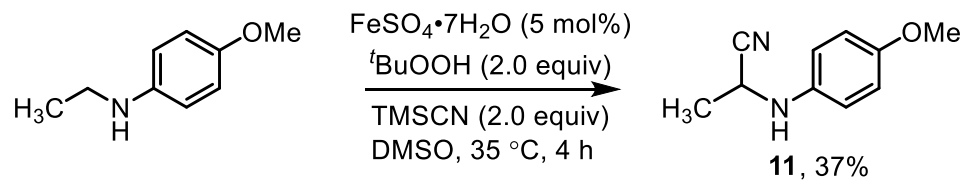

2-((4-Methoxyphenyl)amino)propanenitrile (11): compound 11 was prepared through an Fe-catalyzed oxidative coupling method ${ }^{[17]}\left(37 \%\right.$ yield, yellow solid, m.p. $\left.51-52{ }^{\circ} \mathrm{C}\right) .{ }^{1} \mathrm{H}$ NMR $(400 \mathrm{MHz}$, 
Chloroform- $d$ ) $\delta 6.81-6.73(\mathrm{~m}, 2 \mathrm{H}), 6.68-6.60(\mathrm{~m}, 2 \mathrm{H}), 4.16(\mathrm{q}, J=7.0 \mathrm{~Hz}, 2 \mathrm{H}), 3.70(\mathrm{~s}, 3 \mathrm{H}), 1.61(\mathrm{~d}$, $J=7.0 \mathrm{~Hz}, 3 \mathrm{H}) ;{ }^{13} \mathrm{C}\left\{{ }^{1} \mathrm{H}\right\} \mathrm{NMR}\left(101 \mathrm{MHz}, \mathrm{CDCl}_{3}\right) \delta 154.1,138.6,120.3,116.2,115.0,55.7,42.3,19.8$; IR $v_{\max }\left(\right.$ neat) $/ \mathrm{cm}^{-1}: 3369,3321,2936,2246,1513,1236,1031,825 ;$ HRMS (ESI, m/z): calcd for $\mathrm{C}_{10} \mathrm{H}_{13} \mathrm{~N}_{2} \mathrm{O}^{+}[\mathrm{M}+\mathrm{H}]^{+}$177.1022, found 177.1025 .

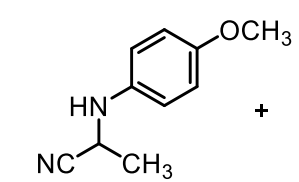

11 ( 1.0 equiv) (91\% recovered)

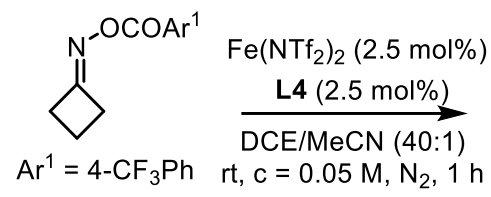

$\mathbf{2 a}$ (1.0 equiv) $(87 \%$ recovered $)$

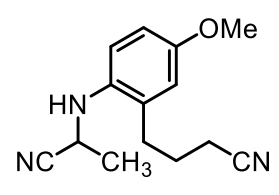

12, NOT detected

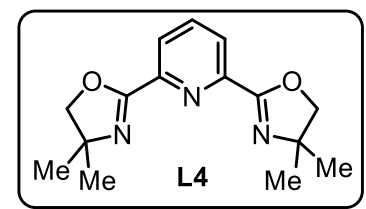

$\mathrm{Me}$

The reaction between $\mathbf{1 1}$ and $\mathbf{2 a}$ was conducted under condition B. The reaction was quenched after the same time, worked-up and then submitted for NMR analysis. Both compound $\mathbf{1 1}$ and $\mathbf{2 a}$ were most recovered without the detection of aryl alkylation product 12. The different reactivity between 9 and 11 suggested that an $\alpha$-amino carbonyl moiety highly likely serves a bidentate ligand, and facilitates the catalytic decomposition of oxime esters $\mathbf{2 a}$.

\section{f) Enantioselective control with chiral Py-BOX ligands}<smiles>COc1ccc(NCC(=O)Nc2ccccc2)cc1</smiles>

10 (1.0 equiv)<smiles>CC1(C)COC(c2cccc(C3=NC(C)(C)CO3)n2)=N1</smiles>

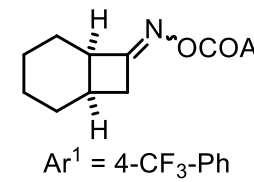

$2 \mathrm{~g}$ (1.2 equiv)<smiles>[R16][R16][R16]</smiles>

$\mathrm{Fe}\left(\mathrm{NTf}_{2}\right)_{2}(2.5 \mathrm{~mol} \%)$ $\underset{\mathrm{DCE} / \mathrm{MeCN}(40: 1)}{\stackrel{\mathrm{L}}{\mathrm{D}}(2.5 \mathrm{~mol} \%)}$ $0^{\circ} \mathrm{C}$ to $\mathrm{rt}, \mathrm{c}=0.05, \mathrm{~N}_{2}, 12 \mathrm{~h}$<smiles>COc1ccc(NC(C(=O)Nc2ccccc2)C2CCCCC2)cc1</smiles>

$\begin{array}{cc}\text { Ligand } & \text { Yield } \\ \text { L4 } & 84 \%, d r=2.8: 1 \\ (1 R, 2 S) \text {-L5 } & 67 \%, d r=2.8: 1 \\ (1 S, 2 R)-\text {-L5 } & 64 \%, d r=2.7: 1\end{array}$

\section{2-(2-(Cyanomethyl)cyclohexyl)-2-((4-methoxyphenyl)amino)-N-phenylacetamide (3og):}

Major diastereomer: Under condition B, compound 3og was prepared by reacting 10 with $\mathbf{2 g}$, and the major diastereomer was isolated through a silica gel flash column (PE: EtOAc from 5:1 to 3:1) as yellowish oil (47 mg, 62\% yield). ${ }^{1} \mathrm{H}$ NMR (400 MHz, Chloroform- $d$ ) $\delta 8.77$ (brs, $\left.1 \mathrm{H}\right), 7.55-7.45$ (m, 2H), 7.35 - $7.27(\mathrm{~m}, 2 \mathrm{H}), 7.17$ - 7.07 (m, 1H), 6.85 - $6.75(\mathrm{~m}, 2 \mathrm{H}), 6.69-6.59$ (m, 2H), 3.80 (br, 1H), $3.77(\mathrm{~d}, J=3.5 \mathrm{~Hz}, 1 \mathrm{H}), 3.74(\mathrm{~s}, 3 \mathrm{H}), 3.00(\mathrm{dd}, J=17.2,6.2 \mathrm{~Hz}, 1 \mathrm{H}), 2.57$ (dd, $J=17.2,3.4 \mathrm{~Hz}, 1 \mathrm{H})$, $2.09-1.98(\mathrm{~m}, 2 \mathrm{H}), 1.92-1.73(\mathrm{~m}, 4 \mathrm{H}), 1.49-1.14(\mathrm{~m}, 4 \mathrm{H}) ;{ }^{13} \mathrm{C}\left\{{ }^{1} \mathrm{H}\right\} \mathrm{NMR}(101 \mathrm{MHz}$, Chloroform- $d$ ) $\delta 169.8,153.8,140.4,137.0,129.0,124.6,119.9,118.4,115.3,115.0,61.6,55.7,46.0,35.9,32.5,27.4$, 25.8, 25.5, 22.5; IR $v_{\max }$ (neat)/cm $\mathrm{cm}^{-1}: 3334,2927,2855,2244,1670,1599,1508,1440,1235,1178,1035$; HRMS (ESI, m/z): calcd for $\mathrm{C}_{23} \mathrm{H}_{28} \mathrm{~N}_{3} \mathrm{O}_{2}{ }^{+}[\mathrm{M}+\mathrm{H}]^{+} 378.2176$, found 378.2176. 
Minor diastereomer: Under condition B, compound 3og was prepared by reacting 10 with $\mathbf{2 g}$, and the minor diastereomer was isolated through a silica gel flash column (PE: EtOAc from 3:1 to 2:1) as white solid (17 mg, 22\% yield, m.p. $185-186{ }^{\circ} \mathrm{C}$ ). ${ }^{1} \mathrm{H}$ NMR (400 MHz, Chloroform- $d$ ) $\delta 8.85$ (brs, 1H), 7.60 $-7.50(\mathrm{~m}, 2 \mathrm{H}), 7.35-7.28(\mathrm{~m}, 2 \mathrm{H}), 7.15-7.08(\mathrm{~m}, 1 \mathrm{H}), 6.85-6.75(\mathrm{~m}, 2 \mathrm{H}), 6.71-6.63(\mathrm{~m}, 2 \mathrm{H}), 3.79$ $(\mathrm{d}, J=3.5 \mathrm{~Hz}, 1 \mathrm{H}), 3.75(\mathrm{~s}, 3 \mathrm{H}), 2.32(\mathrm{dd}, J=16.8,3.8 \mathrm{~Hz}, 1 \mathrm{H}), 2.27-2.03(\mathrm{~m}, 3 \mathrm{H}), 1.90-1.70$ (m, 4H), $1.40-1.27(\mathrm{~m}, 4 \mathrm{H}) ;{ }^{13} \mathrm{C}\left\{{ }^{1} \mathrm{H}\right\} \mathrm{NMR}$ (101 MHz, Chloroform- $d$ ) $\delta$ 170.7, 153.9, 140.4, 137.2, 129.0, 124.6, 119.7, 118.0, 115.3, 115.2, 62.2, 55.7, 44.4, 35.8, 32.2, 26.4, 25.68, 25.65, 22.0; IR $v_{\max }$ (neat)/cm 1: 3331, 2927, 2855, 2247, 1668, 1599, 1509, 1442, 1244, 1178, 1036; HRMS (ESI, m/z): calcd for $\mathrm{C}_{23} \mathrm{H}_{28} \mathrm{~N}_{3} \mathrm{O}_{2}^{+}[\mathrm{M}+\mathrm{H}]^{+}$378.2176, found 378.2176.

The asymmetric reactions were carried out through the same procedures, but using chiral ligand $\mathbf{L 5}$ with opposite absolute configurations, respectively. After isolation of the diastereomers, the samples obtained from three different reactions $(\mathbf{L 4},(1 R, 2 S)$-L5 and $(1 S, 2 R)$-L5) are submitted for chiral HPLC analysis. Conditions: Daicel Chiralcel AD-H, Hexanes $/{ }^{i} \mathrm{PrOH}$ 80:20, $0.8 \mathrm{~mL} / \mathrm{min}$, UV detector $254 \mathrm{~nm}$

\begin{tabular}{|l|c|c|c|c|}
\hline & \multicolumn{2}{|c|}{ retention time (min) } & ee & ee \\
& \multicolumn{2}{|l|}{} & $(1 R, 2 S)-\mathbf{L 5}$ & $(1 S, 2 R)-\mathbf{L 5}$ \\
\hline Major isomer & 11.9 & 15.1 & $7 \%$ & $-6 \%$ \\
\hline Minor isomer & 13.8 & 25.3 & $10 \%$ & $-10 \%$ \\
\hline
\end{tabular}

HPLC traces:

1. Major diastereomer, racemic sample with $\mathbf{L 4}$ :

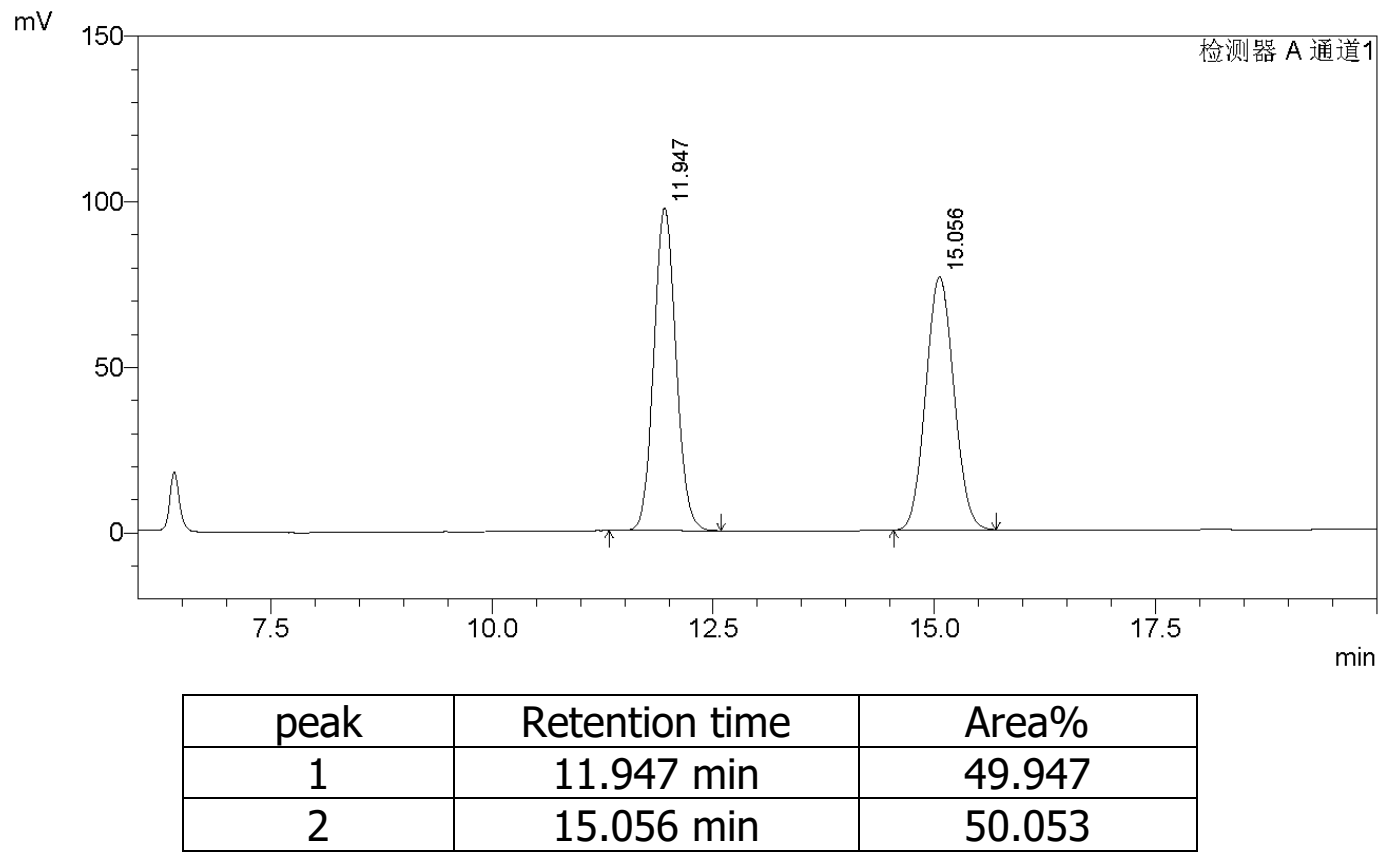


2. Major diastereomer, reaction with $(\mathbf{1 R}, \mathbf{2 S})$-L5:

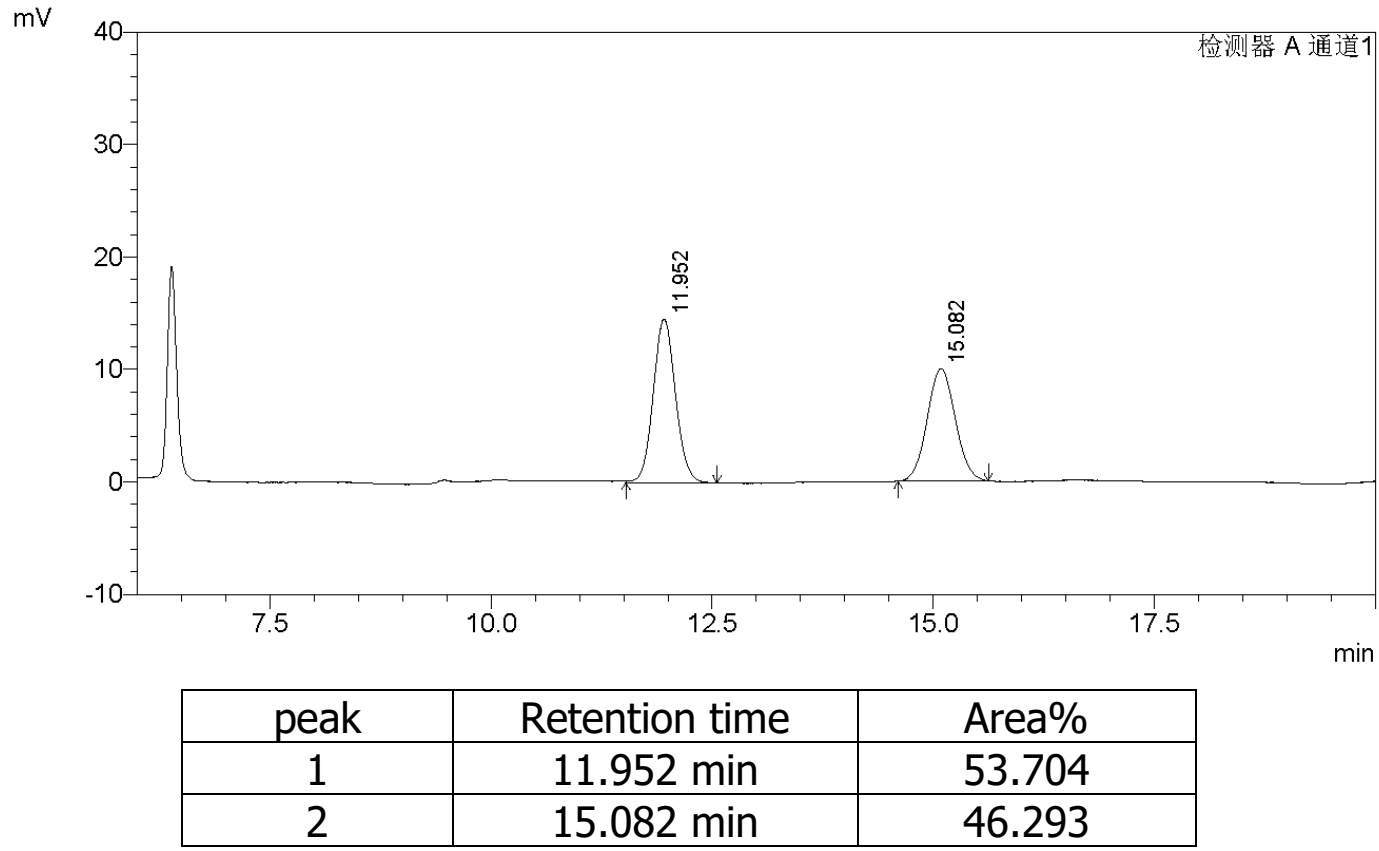

3. Major diastereomer, reaction with $(\mathbf{1 S}, \mathbf{2 R})$-L5:

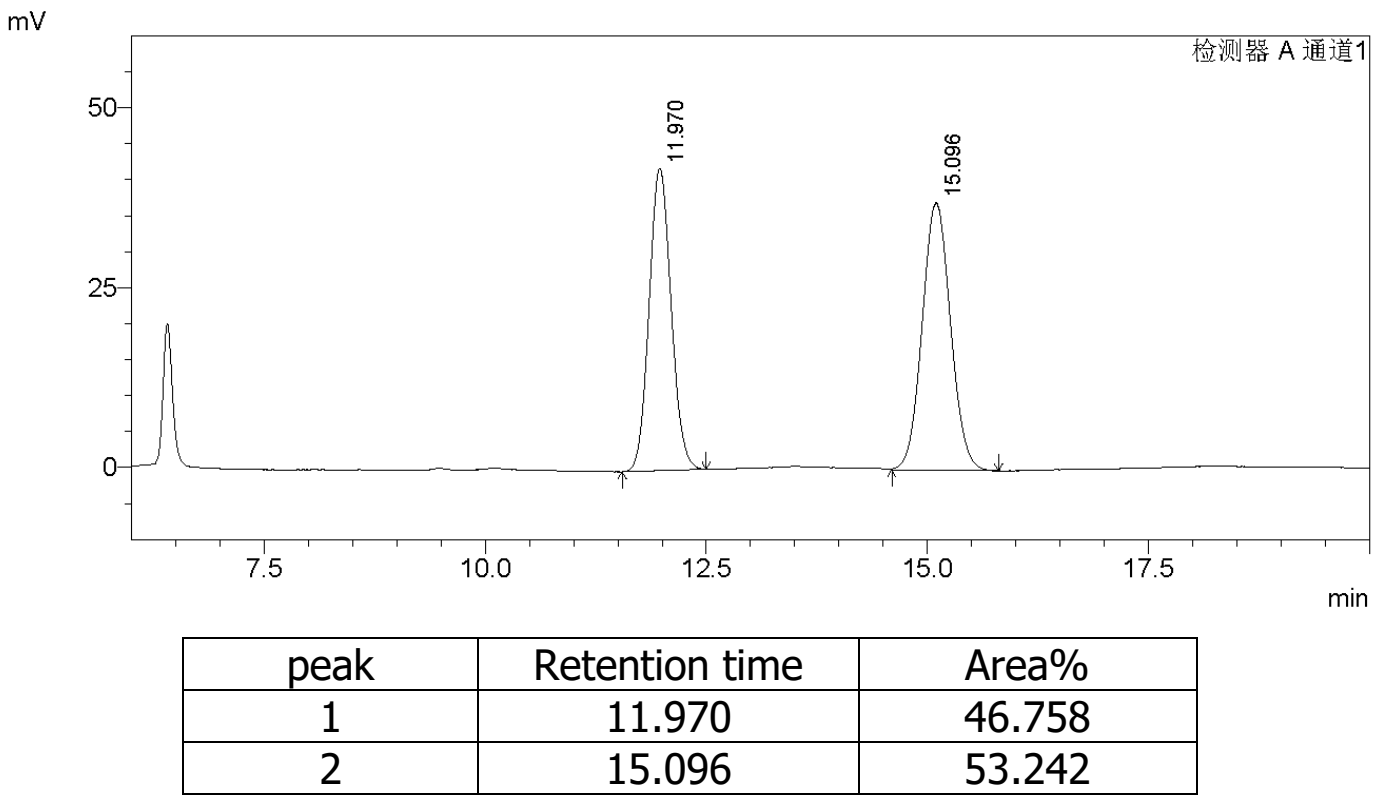


4. Minor diastereomer, racemic sample with $\mathbf{L 4}$

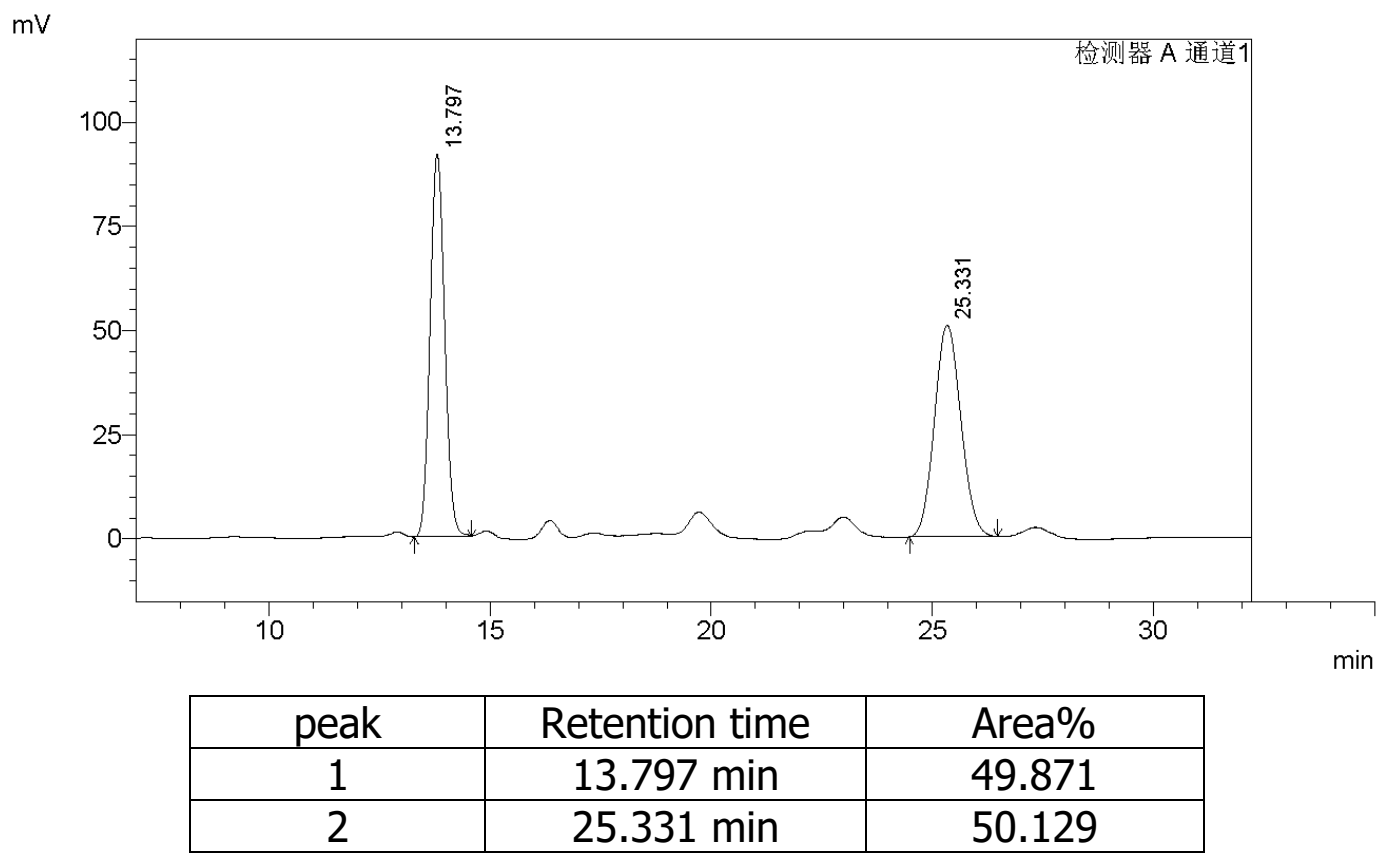

5. Minor diastereomer, reaction with $(\mathbf{1} R, \mathbf{2 S})-\mathbf{L 5}$ :

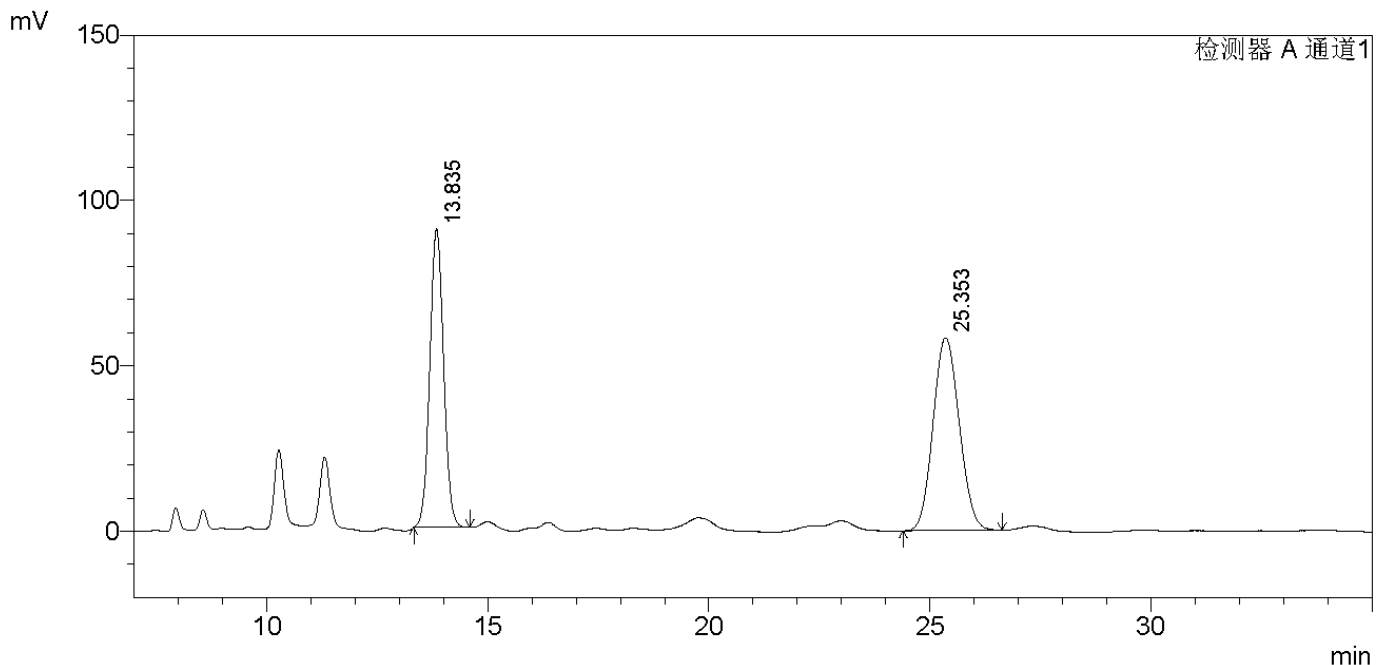

\begin{tabular}{|c|c|c|}
\hline peak & Retention time & Area\% \\
\hline 1 & $13.835 \mathrm{~min}$ & 45.006 \\
\hline 2 & $25.353 \mathrm{~min}$ & 54.994 \\
\hline
\end{tabular}


6. Minor diastereomer, reaction with $(\mathbf{1 S}, \mathbf{2 R})$-L5:

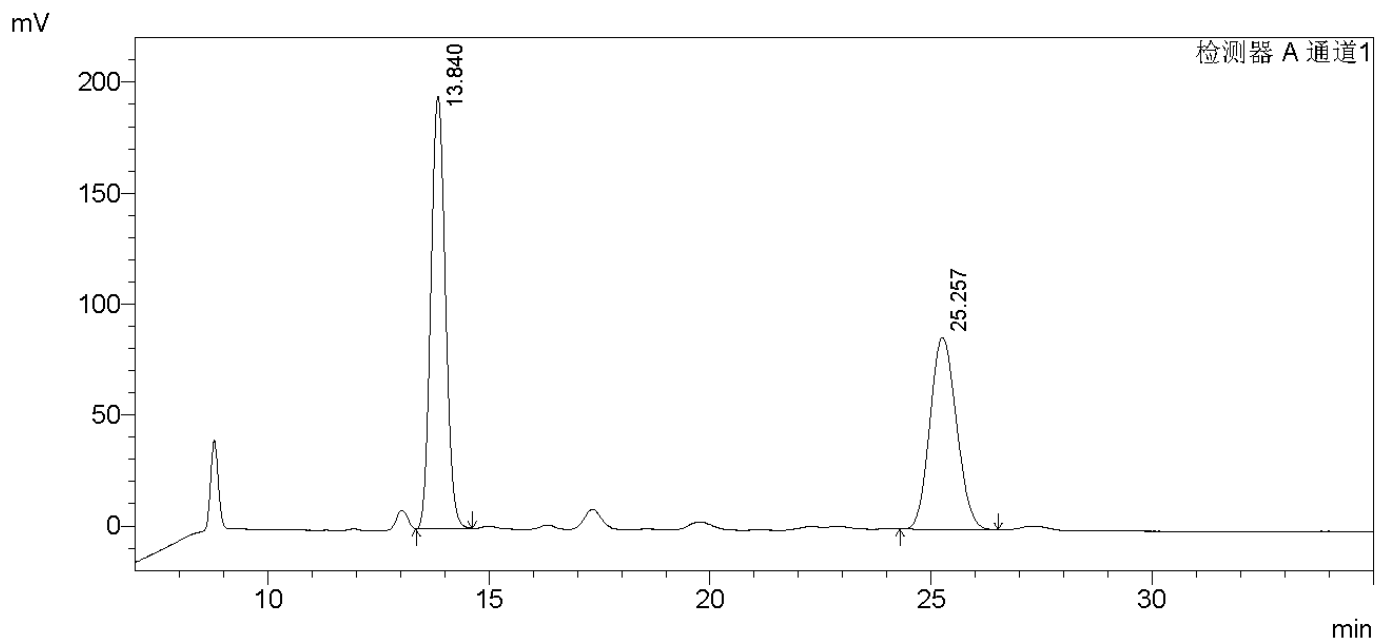

\begin{tabular}{|c|c|c|}
\hline peak & Retention time & Area\% \\
\hline 1 & $13.840 \mathrm{~min}$ & 55.115 \\
\hline 2 & $25.257 \mathrm{~min}$ & 44.885 \\
\hline
\end{tabular}

\section{Gram Scale Synthesis \& Product Derivatizations}

\section{a) Gram Scale Synthesis}
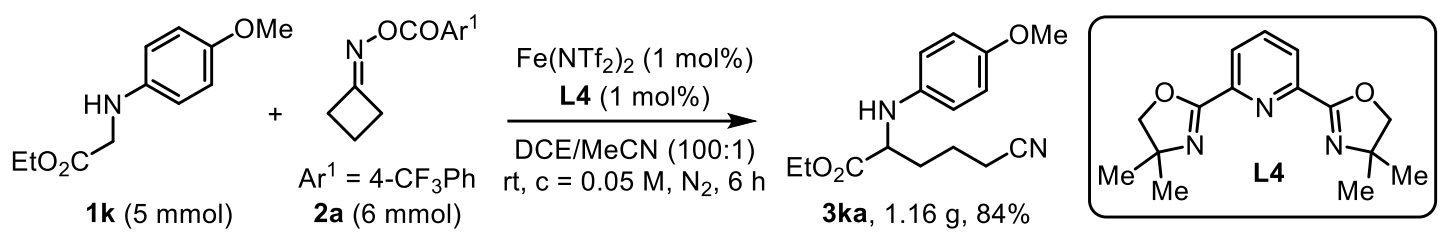

In a $\mathrm{N}_{2}$ atmosphere glovebox, $\mathrm{Fe}\left(\mathrm{NTf}_{2}\right)_{2}(30.8 \mathrm{mg}, 0.05 \mathrm{mmol})$, and $\mathbf{L 4}(13.7 \mathrm{mg}, 0.05 \mathrm{mmol})$ were weighed into a 2-dram vial charged with a stir bar. The vial was sealed under $\mathrm{N}_{2}$ with a septum and taken out of the glovebox. Then, freshly distilled dry 1,2-dichloroethane $(4.0 \mathrm{~mL})$ and dry $\mathrm{MeCN}(1.0 \mathrm{~mL})$ was added into the vial. The mixture was stirred at room temperature to afford a red catalyst solution. Outside the glovebox, to a flame-dried round bottom flask $(200 \mathrm{~mL})$ charged with a stir bar were added ethyl $\mathrm{N}$ PMP glycinate 1 (1.05 g, $5.0 \mathrm{mmol})$ and oxime ester 2a $(1.54 \mathrm{~g}, 6.0 \mathrm{mmol})$. After the flask was evacuated and backfilled with $\mathrm{N}_{2}$ three times, anhydrous 1,2-dichloroethane $(95 \mathrm{~mL})$ was added. The above catalyst solution was added dropwise to the flask while stirring, and the resulting mixture was kept stirred at room temperature $\left(23\right.$ to $26^{\circ} \mathrm{C}$ ) for $6 \mathrm{~h}$. The reaction was quenched with saturated $\mathrm{NaHCO}_{3}$ aqueous solution (40 $\mathrm{mL})$ and the aqueous phase was extracted with $\mathrm{CH}_{2} \mathrm{Cl}_{2}(40 \mathrm{~mL} \times 3)$. The combined organic layers were dried over anhydrous $\mathrm{Na}_{2} \mathrm{SO}_{4}$, filtered, and then concentrated in vacuo to afford the crude product. After a silica gel chromatography (PE: EtOAc, from 10:1 to 5:1), compound 3ka was isolated as yellowish oil $(1.16 \mathrm{~g}, 84 \%$ yield $)$. 


\section{b) The Removal of the $N$-PMP group}

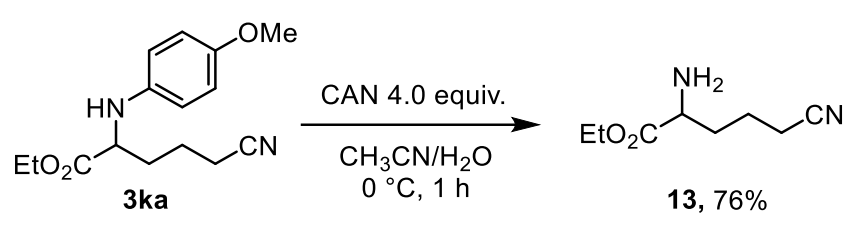

Ethyl 2-amino-5-cyanopentanoate (13): To a solution of CAN (ceric ammonium nitrate, 0.4 mmol, dissolved in $\left.0.7 \mathrm{ml} \mathrm{H}_{2} \mathrm{O}\right)$ stirred at $0{ }^{\circ} \mathrm{C}$ was added a solution of $3 \mathbf{k a}(0.1 \mathrm{mmol}$ dissolved in $0.3 \mathrm{ml}$ $\mathrm{CH}_{3} \mathrm{CN}$ ). The reaction was kept stirring for $1 \mathrm{~h}$ at the same temperature before it was modulated to alkalescence with saturated aqueous $\mathrm{NaHCO}_{3}$. Then the mixture was extracted with $\mathrm{CH}_{2} \mathrm{Cl}_{2}$ for three times, washed with brine, dried over $\mathrm{Na}_{2} \mathrm{SO}_{4}$ and concentrated in vacuo. The residue was purified through a silica gel flash column $\left(\mathrm{CH}_{2} \mathrm{Cl}_{2} / \mathrm{CH}_{3} \mathrm{OH}=20: 1\right)$ to give the product 13 as yellow oil $(13 \mathrm{mg}, 76 \%) ;{ }^{1} \mathrm{H}$ NMR (400 MHz, Chloroform- $d$ ) $\delta 4.18(\mathrm{q}, J=7.2 \mathrm{~Hz}, 2 \mathrm{H}), 3.43(\mathrm{dd}, J=7.9,4.8 \mathrm{~Hz}, 1 \mathrm{H}), 2.41$ (t, $J=6.8$ $\mathrm{Hz}, 2 \mathrm{H}), 1.96-1.61(\mathrm{~m}, 6 \mathrm{H}), 1.28(\mathrm{t}, J=7.1 \mathrm{~Hz}, 3 \mathrm{H}) ;{ }^{13} \mathrm{C}\left\{{ }^{1} \mathrm{H}\right\} \mathrm{NMR}(101 \mathrm{MHz}$, Chloroform- $d$ ) $\delta 175.4$, 119.3, 61.2, 53.8, 33.4, 22.0, 17.0, 14.2; IR $v_{\max }$ (neat)/ $\mathrm{cm}^{-1}: 3370,2980,2936,2869,2245,1730,1516$, 1275, 1260, 1187, 1024; HRMS (ESI, m/z): calcd for $\mathrm{C}_{8} \mathrm{H}_{15} \mathrm{~N}_{2} \mathrm{O}_{2}{ }^{+}[\mathrm{M}+\mathrm{H}]^{+}$171.1128, found 171.1129.

\section{c) The reduction of the ester group}

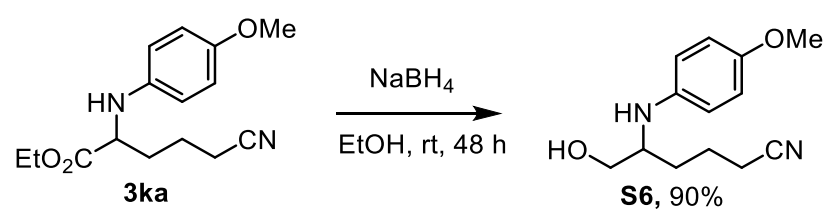

6-Hydroxy-5-((4-methoxyphenyl)amino)hexanenitrile (S6): $\mathrm{NaBH}_{4}(0.5 \mathrm{mmol})$ was added to the solution of $3 \mathbf{k a}(0.1 \mathrm{mmol})$ in $\mathrm{EtOH}(1 \mathrm{~mL})$ at $0{ }^{\circ} \mathrm{C}$. The mixture was warmed to room temperature and stirred for 48 hours. After $\mathbf{3 k a}$ was consumed as indicated by TLC, the reaction was quenched with brine and extracted with $\mathrm{CH}_{2} \mathrm{Cl}_{2}(10 \mathrm{~mL} \times 3)$. The combined organic layers were dried over anhydrous $\mathrm{Na}_{2} \mathrm{SO}_{4}$, and concentrated in vacuo. The residue was purified through a silica gel flash column $(\mathrm{PE} /$ Acetone $=2: 1)$ to give the amino alcohol S6 as yellow oil (21 mg, 90\%). ${ }^{1} \mathrm{H}$ NMR (400 MHz, Chloroform- $d$ ) $\delta 6.81-$ $6.73(\mathrm{~m}, 2 \mathrm{H}), 6.65-6.58(\mathrm{~m}, 2 \mathrm{H}), 3.74(\mathrm{~s}, 3 \mathrm{H}), 3.70(\mathrm{dd}, J=10.8,4.4 \mathrm{~Hz}, 1 \mathrm{H}), 3.51$ (dd, $J=10.8,5.4$ $\mathrm{Hz}, 1 \mathrm{H}), 3.39$ (dt, $J=7.7,4.8 \mathrm{~Hz}, 1 \mathrm{H}), 2.58(\mathrm{br}, 2 \mathrm{H}), 2.35$ (t, $J=6.6 \mathrm{~Hz}, 2 \mathrm{H}), 1.86-1.54(\mathrm{~m}, 4 \mathrm{H})$; ${ }^{13} \mathrm{C}\left\{{ }^{1} \mathrm{H}\right\} \mathrm{NMR}(101 \mathrm{MHz}$, Chloroform- $d$ ) $\delta$ 152.6, 141.3, 119.5, 115.4, 115.1, 64.1, 55.8, 55.7, 31.4, 22.4, 17.3; IR $v_{\max }$ (neat)/cm ${ }^{-1}: 3647,3369,2933,2834,2245,1509,1456,1233,1179,1034$; HRMS (ESI, $\mathrm{m} / \mathrm{z}$ ): calcd for $\mathrm{C}_{13} \mathrm{H}_{19} \mathrm{~N}_{2} \mathrm{O}_{2}{ }^{+}[\mathrm{M}+\mathrm{H}]^{+} 235.1141$, found 235.1142 .

\section{d) The hydrolysis of the cyano group}



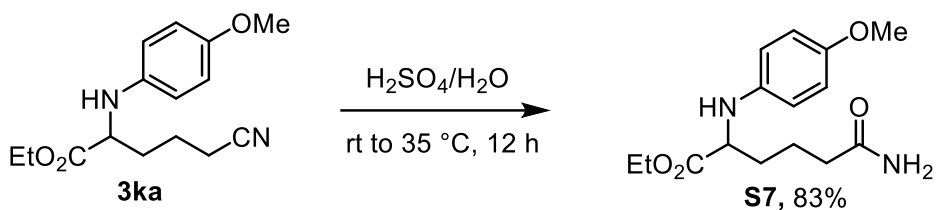

Ethyl 6-amino-2-((4-methoxyphenyl)amino)-6-oxohexanoate (S7): To a solution of 3ka (0.1 mmol, dissolved in $1.0 \mathrm{~mL}$ of toluene) was added a cooled aqueous solution of sulfuric acid ( $8.0 \mu \mathrm{L} \mathrm{H}_{2} \mathrm{SO}_{4}$ dissolved in $50.0 \mu \mathrm{L} \mathrm{H}_{2} \mathrm{O}$ ). The biphasic mixture was stirred at room temperature for $0.5 \mathrm{~h}$, warmed to 35 ${ }^{\circ} \mathrm{C}$, and stirred for additional $12 \mathrm{~h}$. The reaction was cooled to room temperature and quenched with a $\mathrm{Na}_{2} \mathrm{CO}_{3}$ solution ( $35 \mathrm{mg}$ in $1 \mathrm{~mL}$ of $\mathrm{H}_{2} \mathrm{O}$ ). The organic layer was separated and the aqeous one was extracted with EtOAc $(2 \mathrm{~mL} \times 2)$. The combined organic layers were washed with brine, dried over $\mathrm{NaSO}_{4}$ and concentrated in vacuo. The residue was purified through a silica gel flash column (PE/Acetone $=1: 1)$ to give the product $\mathbf{S 7}$ as yellow solid $\left(24 \mathrm{mg}, 83 \%\right.$ yield, m.p. $\left.80-81{ }^{\circ} \mathrm{C}\right) .{ }^{1} \mathrm{H} \mathrm{NMR}(400 \mathrm{MHz}$, Chloroform- $d$ ) $\delta 6.79-6.70(\mathrm{~m}, 2 \mathrm{H}), 6.63-6.54(\mathrm{~m}, 2 \mathrm{H}), 5.74$ (brs, 1H), 5.61 (brs, 1H), 4.15 (q, $J=7.1$ $\mathrm{Hz}, 2 \mathrm{H}), 4.03-3.90(\mathrm{~m}, 1 \mathrm{H}), 3.72(\mathrm{~s}, 3 \mathrm{H}), 2.25(\mathrm{t}, J=6.6 \mathrm{~Hz}, 2 \mathrm{H}), 1.92-1.70(\mathrm{~m}, 4 \mathrm{H}), 1.22(\mathrm{t}, J=7.1$ $\mathrm{Hz}, 3 \mathrm{H}) ;{ }^{13} \mathrm{C}\left\{{ }^{1} \mathrm{H}\right\} \mathrm{NMR}(101 \mathrm{MHz}$, Chloroform- $d) \delta 174.9,174.2,152.8,140.9,115.2,114.9$, 61.1, 57.7, 55.7, 35.2, 32.4, 21.6, 14.2; IR $v_{\max }$ (neat)/ $\mathrm{cm}^{-1}$ : 3351, 3195, 2934, 2831, 1728, 1662, 1621, 1513, 1463, 1410, 1237, 1181, 1034; HRMS (ESI, m/z): calcd for $\mathrm{C}_{15} \mathrm{H}_{23} \mathrm{~N}_{2} \mathrm{O}_{4}{ }^{+}[\mathrm{M}+\mathrm{H}]^{+}$295.1652, found 295.1655.

e) The reduction of the cyano group
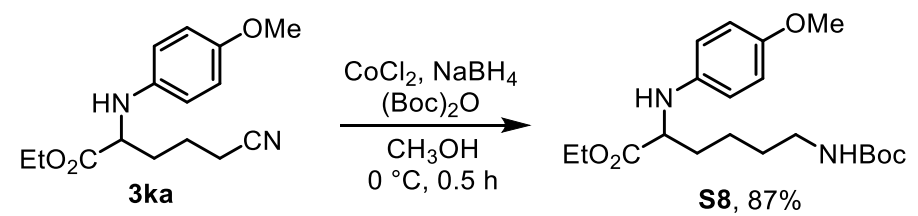

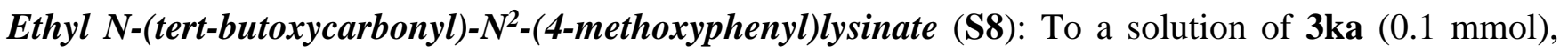
(Boc) $)_{2} \mathrm{O}(0.2 \mathrm{mmol})$ and $\mathrm{CoCl}_{2}(0.2 \mathrm{mmol})$ in $\mathrm{MeOH}(1 \mathrm{~mL})$ was added $\mathrm{NaBH}_{4}(0.3 \mathrm{mmol})$ in portions at $0{ }^{\circ} \mathrm{C}$. The reaction was stirred for $0.5 \mathrm{~h}$ at the same temperature and then quenched with water $(5 \mathrm{~mL})$. The resulting mixture was filtered over celite and the insoluble was washed with $\mathrm{CH}_{2} \mathrm{Cl}_{2}(2 \mathrm{~mL} \times 3)$. After separation and extraction with $\mathrm{CH}_{2} \mathrm{Cl}_{2}(2 \mathrm{~mL} \times 2)$, the combined organic layers were washed with brine, dried over $\mathrm{NaSO}_{4}$ and concetration in vacuo. The residue was purified through a silica gel flash column (PE/Acetone $=3: 1)$ to give the $N$-Boc protected lycinate $\mathbf{S 8}$ as colorless oil (33 mg, 87\%). ${ }^{1} \mathrm{H}$ NMR (400 MHz, Chloroform- $d$ ) $\delta 6.80-6.71(\mathrm{~m}, 2 \mathrm{H}), 6.64-6.55(\mathrm{~m}, 2 \mathrm{H}), 4.56$ (brs, 1H), $4.15(\mathrm{q}, J=7.1 \mathrm{~Hz}, 2 \mathrm{H})$, $3.94(\mathrm{t}, J=6.6 \mathrm{~Hz}, 1 \mathrm{H}), 3.86(\mathrm{br}, 1 \mathrm{H}), 3.72(\mathrm{~s}, 3 \mathrm{H}), 3.16-3.03(\mathrm{~m}, 1 \mathrm{H}), 1.89-1.67$ (m, 2H), $1.56-1.44$ $(\mathrm{m}, 4 \mathrm{H}), 1.40(\mathrm{~s}, 13 \mathrm{H}), 1.22(\mathrm{t}, J=7.1 \mathrm{~Hz}, 3 \mathrm{H}) ;{ }^{13} \mathrm{C}\left\{{ }^{1} \mathrm{H}\right\} \mathrm{NMR}(101 \mathrm{MHz}$, Chloroform- $d) \delta$ 174.4, 156.0, 152.7, 141.0, 115.1, 114.9, 79.1, 61.0, 57.8, 55.7, 40.3, 32.8, 29.8, 28.4, 22.9, 14.3; IR $v_{\max }(\mathrm{neat}) / \mathrm{cm}^{-1}$ : 3370, 2976, 2934, 1698, 1514, 1455, 1393, 1365, 1238, 1174, 1037; HRMS (ESI, m/z): calcd for $\mathrm{C}_{20} \mathrm{H}_{33} \mathrm{~N}_{2} \mathrm{O}_{5}{ }^{+}[\mathrm{M}+\mathrm{H}]^{+}$381.2384, found 381.2388. 


\section{References}

[1] Roberts, D. A.; Pilgrim, B. S.; Cooper, J. D.; Ronson, T. K.; Zarra, S.; Nitschke, J. R., Post-assembly Modification of Tetrazine-Edged Fe ${ }_{4}{ }_{4} \mathrm{~L}_{6}$ Tetrahedra. J. Am. Chem. Soc. 2015, 137, 10068-10071.

[2] Sibi, M. P.; Petrovic, G., Enantioselective radical reactions: the use of metal triflimides as Lewis acids. Tetrahedron: Asymmetry 2003, 14, 2879-2882.

[3] Xu, Q.; Li, B.; Ma, Y.; Sun, F.; Gao, Y.; Ye, N., $\mathrm{K}_{2} \mathrm{~S}_{2} \mathrm{O}_{8}$-HFIP synergistically promoted para-selective sp3 C-H bond diarylation of glycine esters. Org. Biomol. Chem. 2020, 18, 666-670.

[4] Tian, H.; Xu, W.; Liu, Y.; Wang, Q., Unnatural $\alpha$-Amino Acid Synthesized through $\alpha$-Alkylation of Glycine Derivatives by Diacyl Peroxides. Org. Lett. 2020, 22, 5005-5008.

[5] Aggarwal, R.; Kumar, R.; Kumar, V., A facile and rapid one-pot synthesis of 1,4-diaryl-2mercaptoimidazoles under solvent-free conditions. J. Sulphur Chem. 2007, 28, 617-623.

[6] Rohlmann, R.; Stopka, T.; Richter, H.; García Mancheño, O., Iron-Catalyzed Oxidative Tandem Reactions with TEMPO Oxoammonium Salts: Synthesis of Dihydroquinazolines and Quinolines. J. Org. Chem. 2013, 78, 6050-6064.

[7] Sun, B.; Yang, J.; Zhang, L.; Shi, R.; Zhang, X.; Xu, T.; Zhuang, X.; Zhu, R.; Yu, C.; Jin, C., Photocatalytic Aerobic Double Friedel-Crafts Reaction of Glycine Derivatives with Anilines: An Efficient Synthesis of Diarylmethanes. Asian J. Org. Chem. 2019, 8, 2058-2064.

[8] Sonobe, T.; Oisaki, K.; Kanai, M., Catalytic aerobic production of imines en route to mild, green, and concise derivatizations of amines. Chem. Sci. 2012, 3, 3249-3255.

[9] Zhang, M.; Imm, S.; Bähn, S.; Neumann, H.; Beller, M., Synthesis of $\alpha$-Amino Acid Amides: Ruthenium-Catalyzed Amination of a-Hydroxy Amides. Angew. Chem. Int. Ed. 2011, 50, 11197-11201. [10] Wang, C.; Guo, M.; Qi, R.; Shang, Q.; Liu, Q.; Wang, S.; Zhao, L.; Wang, R.; Xu, Z., Visible-LightDriven, Copper-Catalyzed Decarboxylative $\mathrm{C}_{(\mathrm{sp} 3)}-\mathrm{H}$ Alkylation of Glycine and Peptides. Angew. Chem. Int. Ed. 2018, 57, 15841-15846.

[11] Inokuma, T.; Suzuki, Y.; Sakaeda, T.; Takemoto, Y., Synthesis of Optically Active N-Aryl Amino Acid Derivatives through the Asymmetric Petasis Reaction Catalyzed by a Novel Hydroxy-Thiourea Catalyst. Chem. Asian J. 2011, 6, 2902-2906.

[12] He, B.-Q.; Yu, X.-Y.; Wang, P.-Z.; Chen, J.-R.; Xiao, W.-J., A photoredox catalyzed iminyl radicaltriggered $\mathrm{C}-\mathrm{C}$ bond cleavage/addition/Kornblum oxidation cascade of oxime esters and styrenes: synthesis of ketonitriles. Chem. Commun. 2018, 54, 12262-12265.

[13] Jian, Y.; Chen, M.; Yang, C.; Xia, W.-j., Minisci-Type C-H Cyanoalkylation of Heteroarenes Through N-O/C-C Bonds Cleavage. Eur. J. Org. Chem. 2020, 2020, 1439-1442.

[14] Zhang, M.-M.; Li, S.-H.; Tu, J.-L.; Min, Q.-Q.; Liu, F., Metal-free iminyl radical-mediated C-C single bond cleavage/functionalization of redox-active oxime esters. Org. Chem. Front. 2020, 7, 622-627. 
[15] Min, Q.-Q.; Li, N.; Chen, G.-L.; Liu, F., Copper-catalysed C(sp3)-N coupling initiated by selective $\mathrm{C}-\mathrm{C}$ bond cleavage of cyclobutanone oxime esters. Org. Chem. Front. 2019, 6, 1200-1204.

[16] Zhao, B.; Shi, Z., Copper-Catalyzed Intermolecular Heck-Like Coupling of Cyclobutanone Oximes Initiated by Selective C-C Bond Cleavage. Angew. Chem. Int. Ed. 2017, 56, 12727-12731.

[17] Huang, M.; Deng, Q.; Gao, Q.; Shi, J.; Zhang, X.; Xiong, Y., Iron-Sulfate-Catalyzed Direct Dehydrogenative Coupling Cyanation of Secondary Phenylamines. Asian J. Org. Chem. 2018, 7, 404410. 


\section{Copies of NMR Spectra for New Compounds}

$\underbrace{0}$

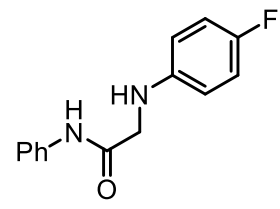

1c $\left(\mathrm{CDCl}_{3}, 400 \mathrm{MHz}\right)$

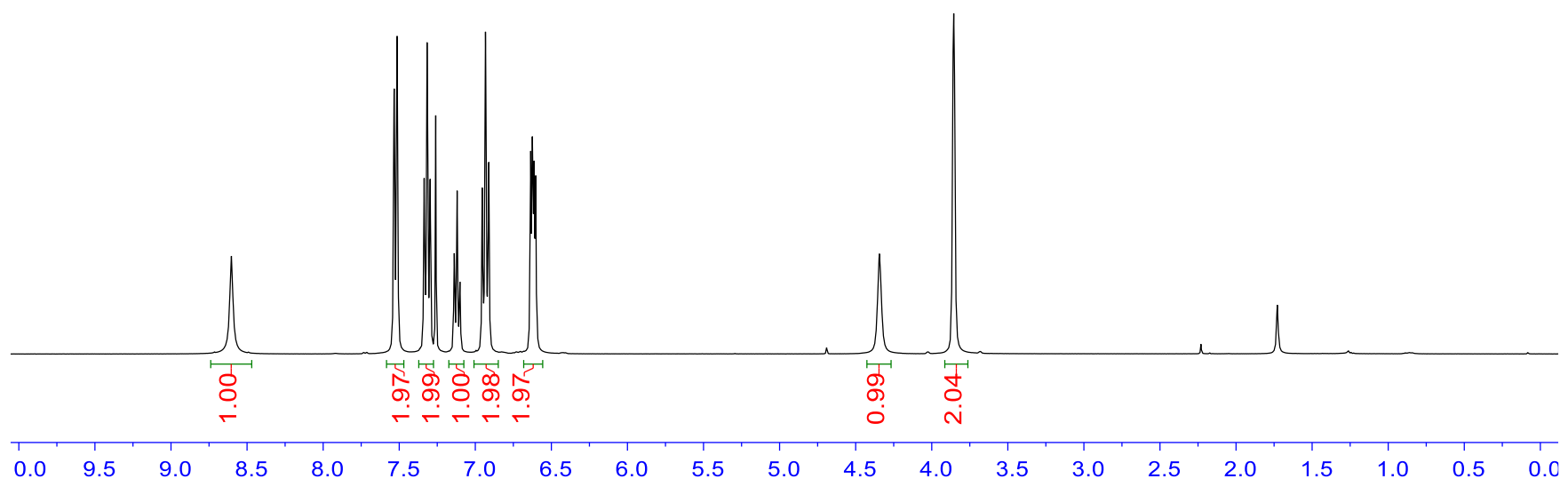

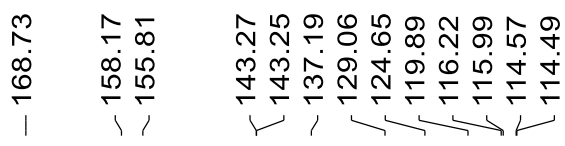

m

เ̊

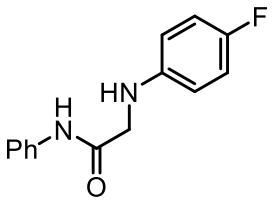

1c $\left(\mathrm{CDCl}_{3}, 101 \mathrm{MHz}\right)$

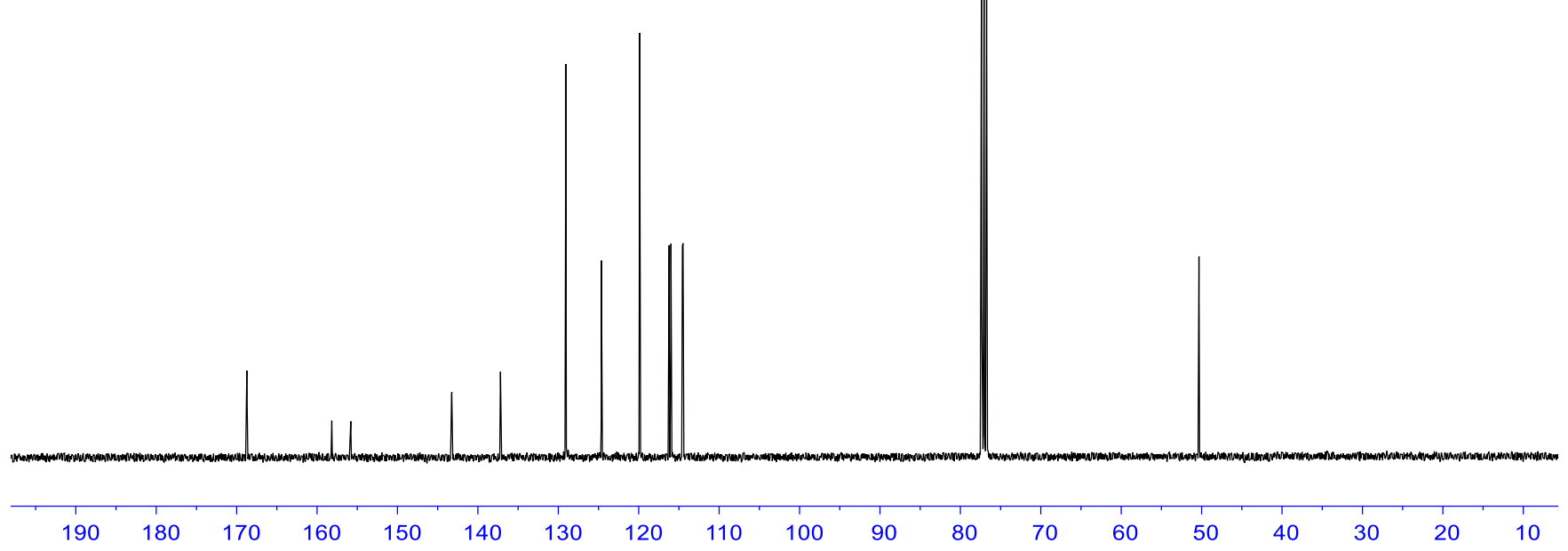




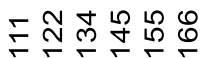

药

(N)

1c $\left(\mathrm{CDCl}_{3}, 376 \mathrm{MHz}\right)$

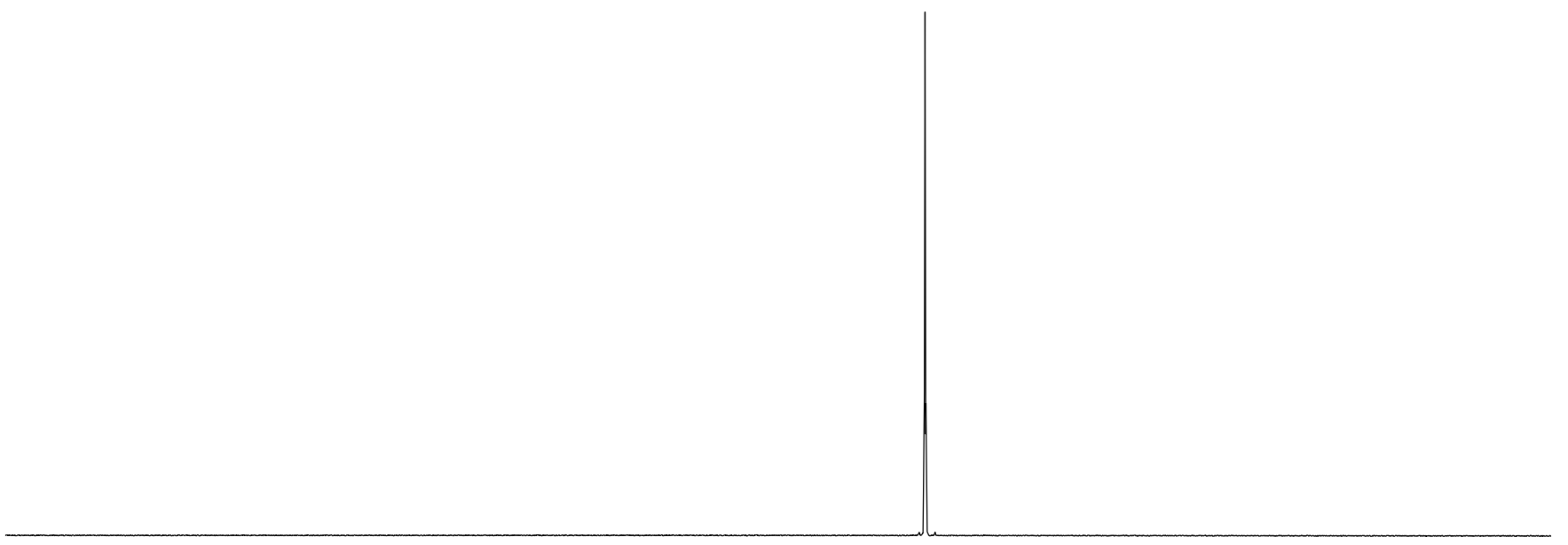

\begin{tabular}{llllllllllllll|l|l|l|l|l|l|l}
\hline-92 & -96 & -100 & -104 & -108 & -112 & -116 & -120 & -124 & -128 & -132 & -136 & -140 & -144 & -148
\end{tabular}




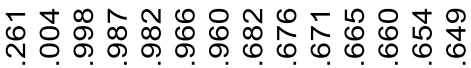

N

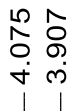

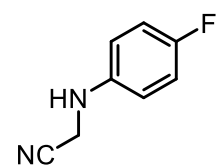

1d $\left(\mathrm{CDCl}_{3}, 400 \mathrm{MHz}\right)$
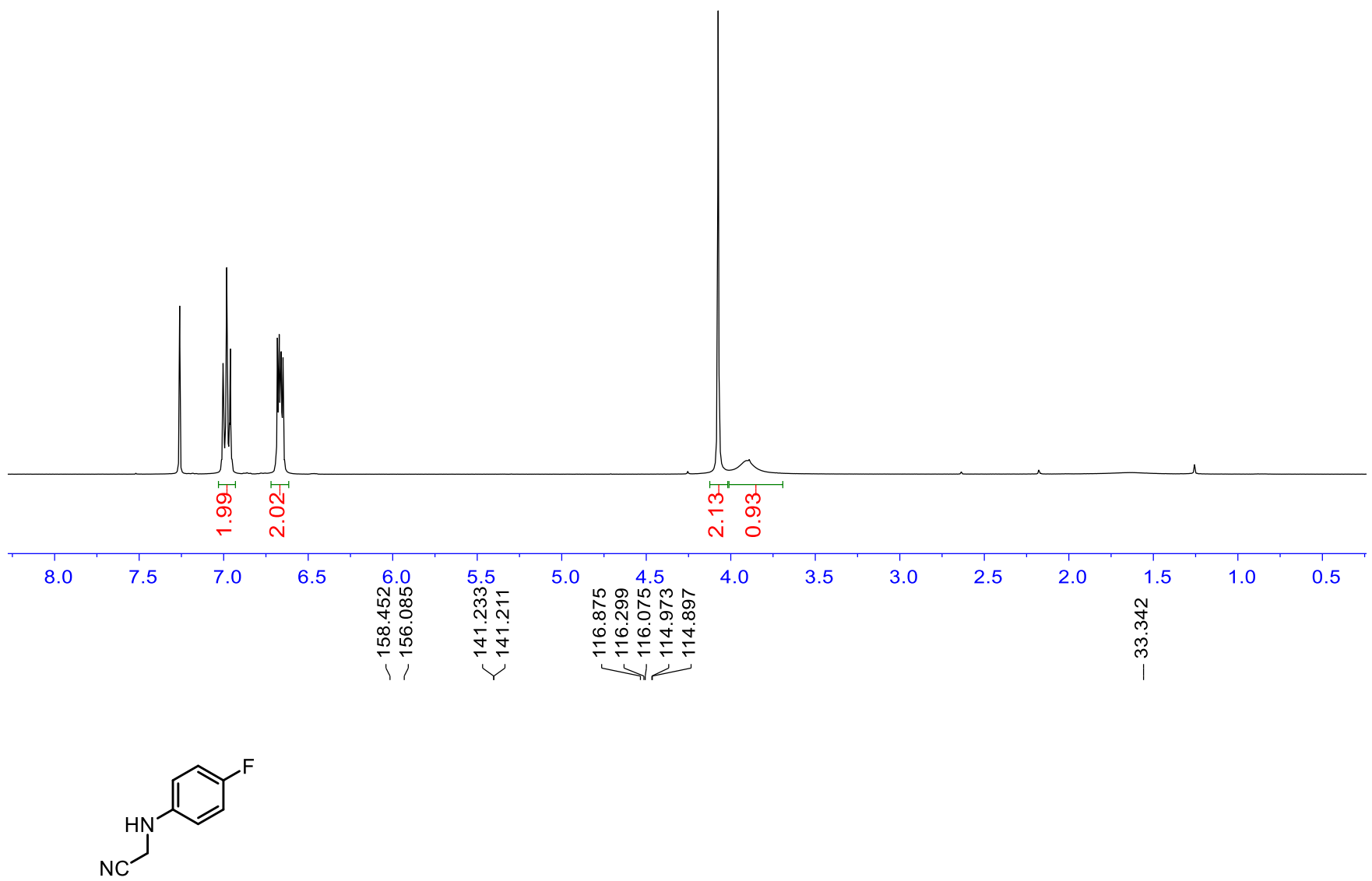

1d $\left(\mathrm{CDCl}_{3}, 101 \mathrm{MHz}\right)$

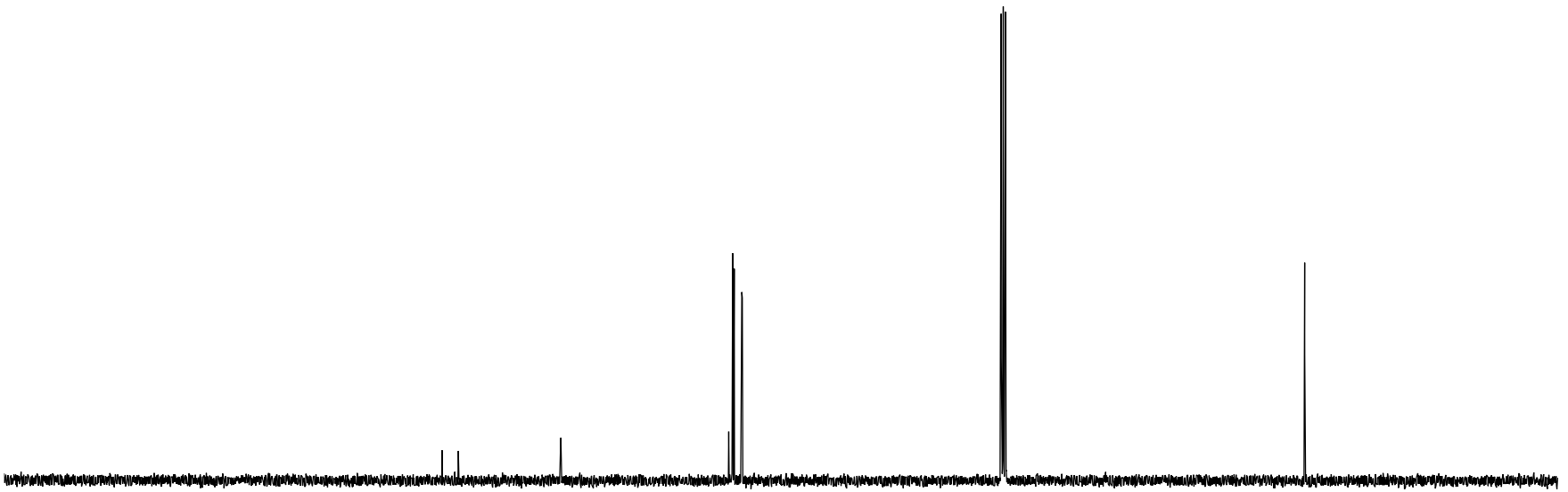

$\begin{array}{lllllllllllllllllllllll}\mid & \mid \\ 220 & 210 & 200 & 190 & 180 & 170 & 160 & 150 & 140 & 130 & 120 & 110 & 100 & 90 & 80 & 70 & 60 & 50 & 40 & 30 & 20 & 10 & 0\end{array}$ 


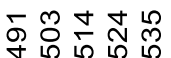

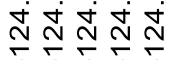

$\mathrm{NC}^{\mathrm{F}}$

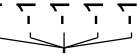

1d $\left(\mathrm{CDCl}_{3}, 376 \mathrm{MHz}\right)$ 

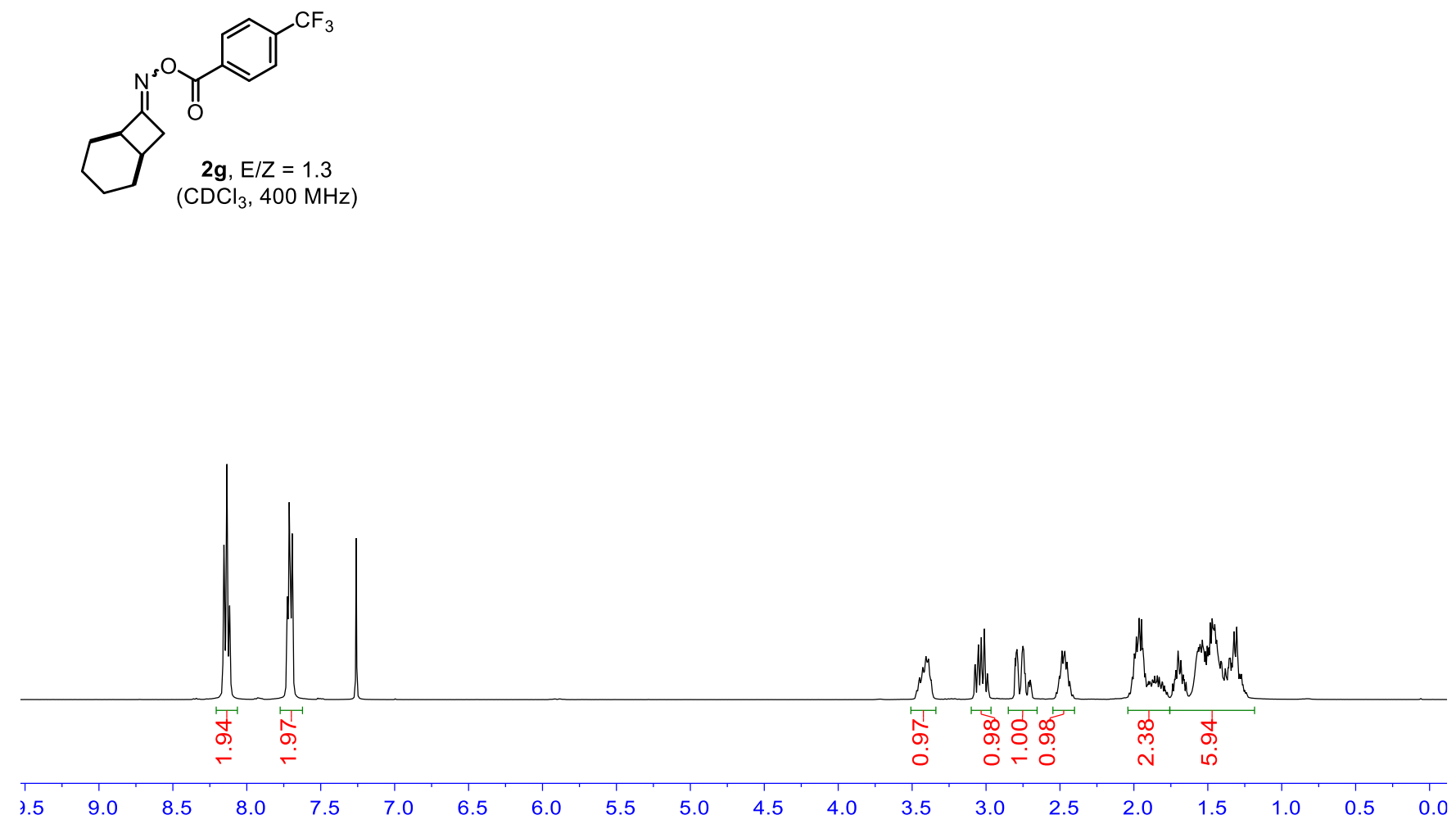

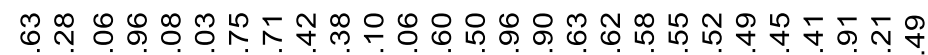

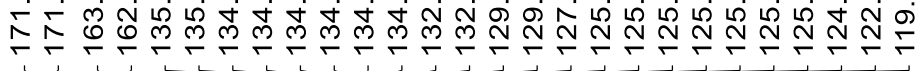

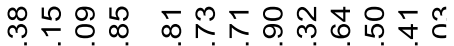

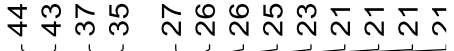
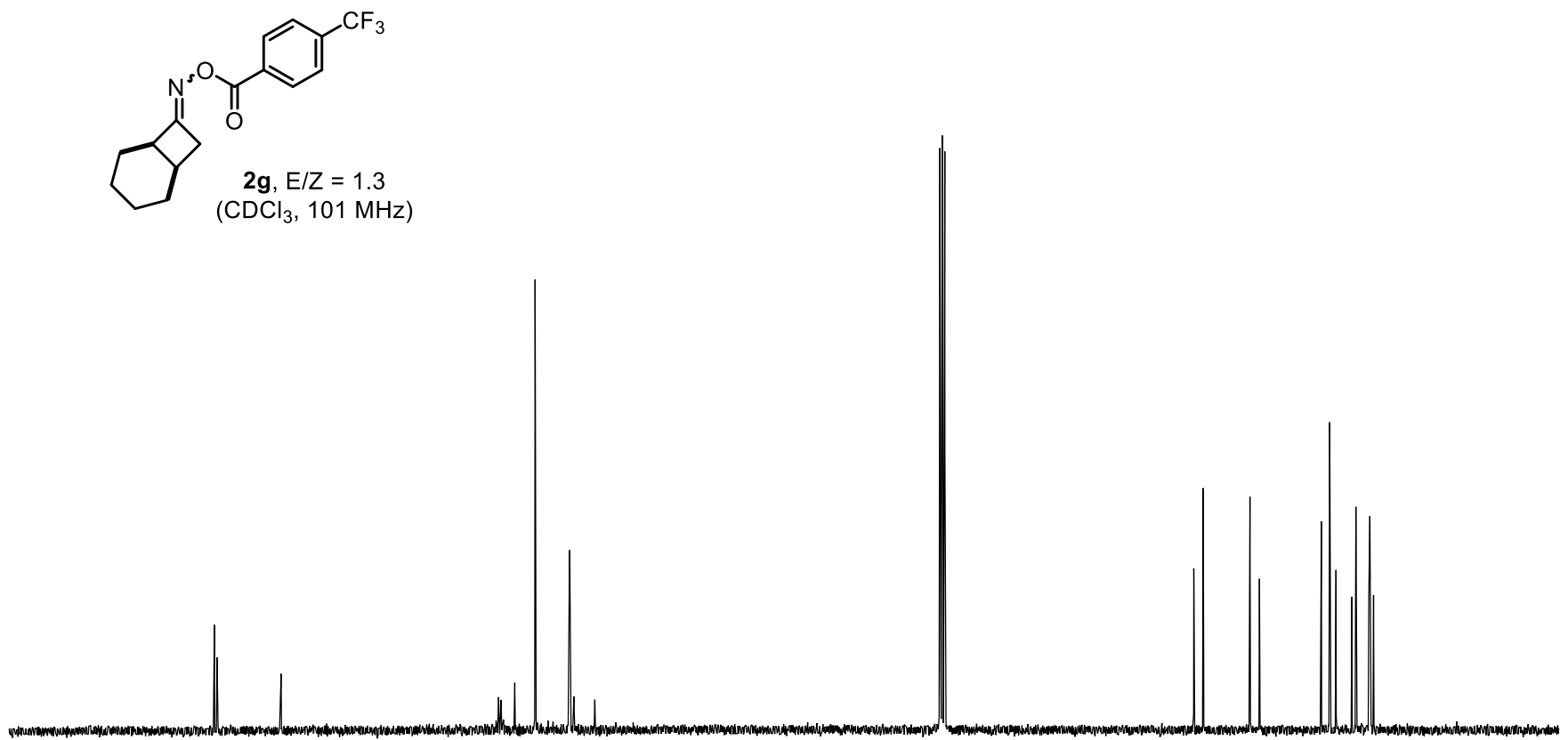

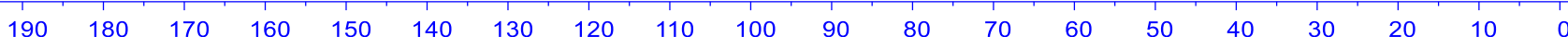




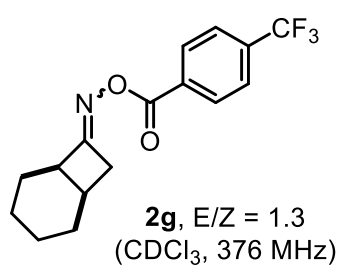

웅욤

\%ं०

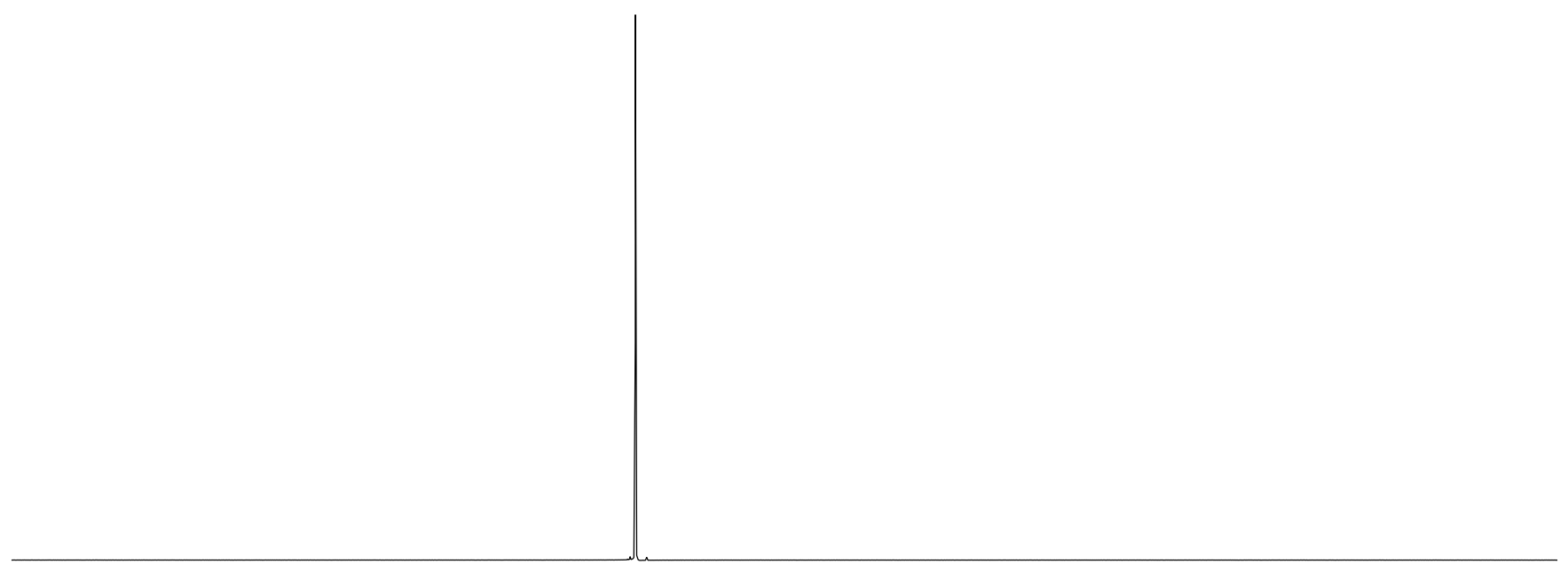

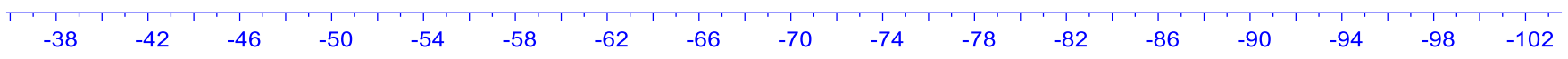

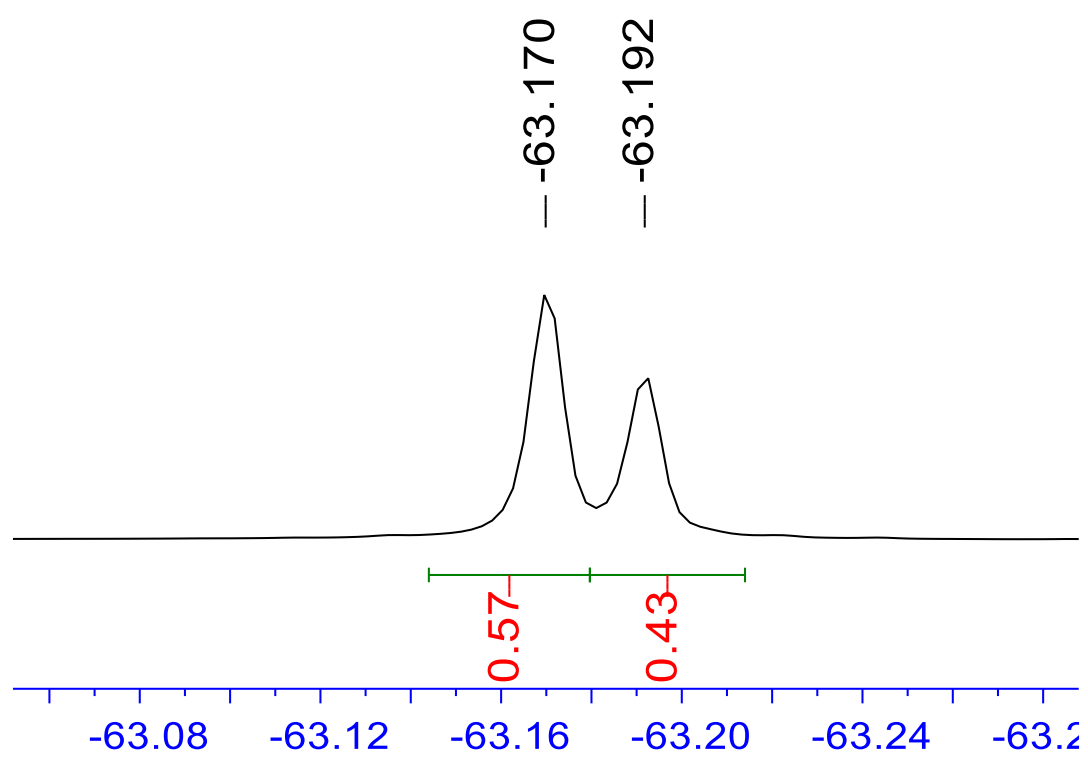




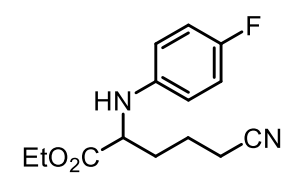

3aa $\left(\mathrm{CDCl}_{3}, 400 \mathrm{MHz}\right)$

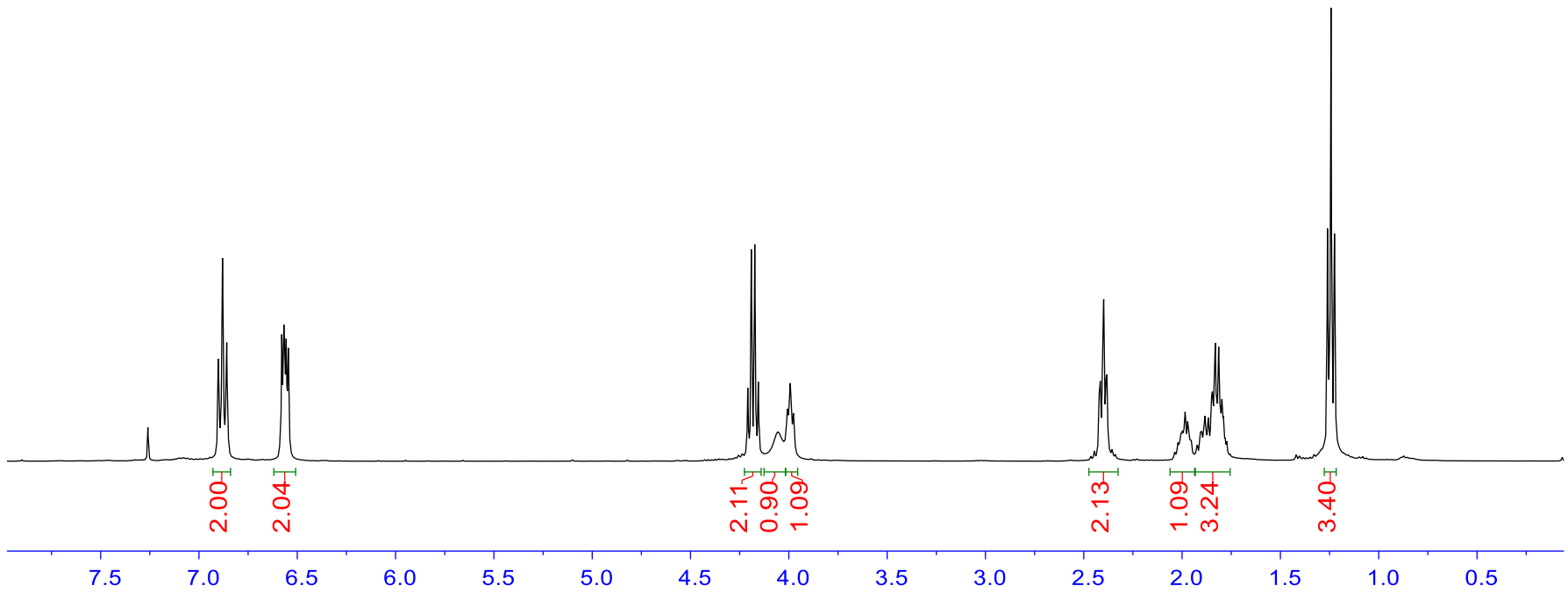

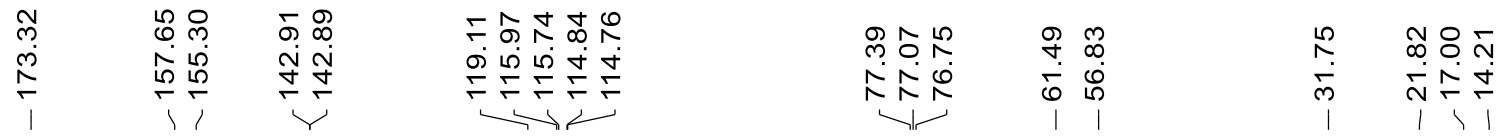

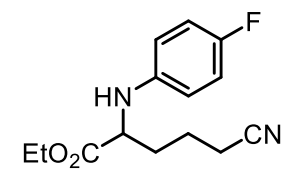

3aa $\left(\mathrm{CDCl}_{3}, 101 \mathrm{MHz}\right)$

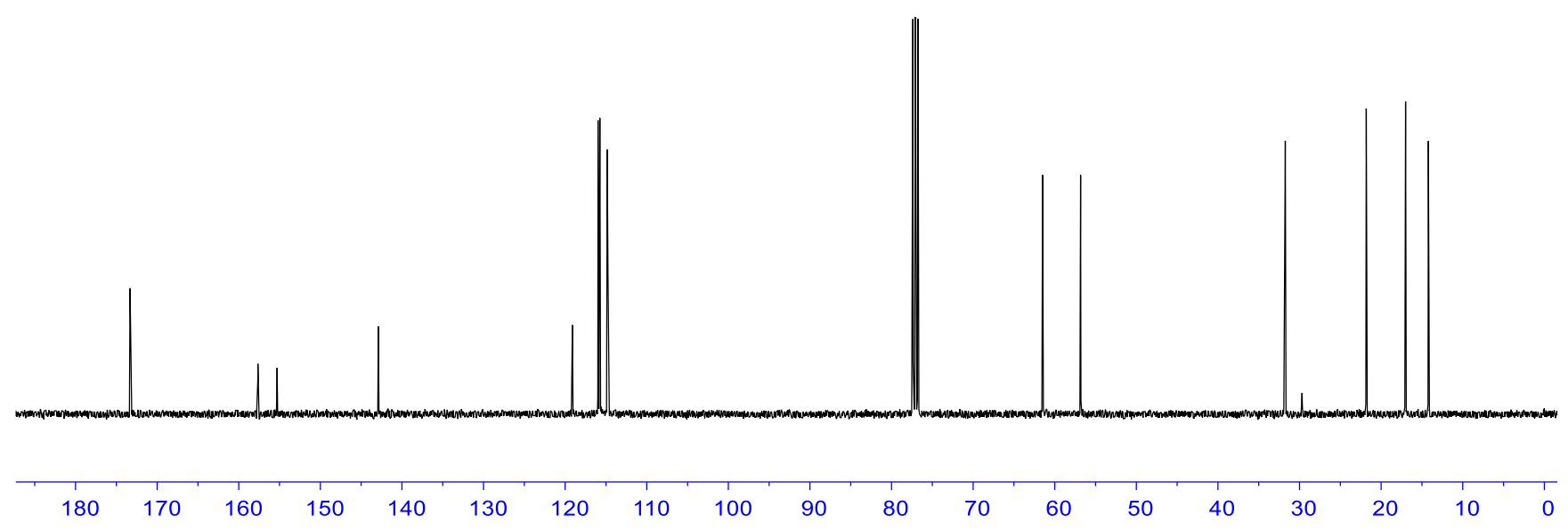




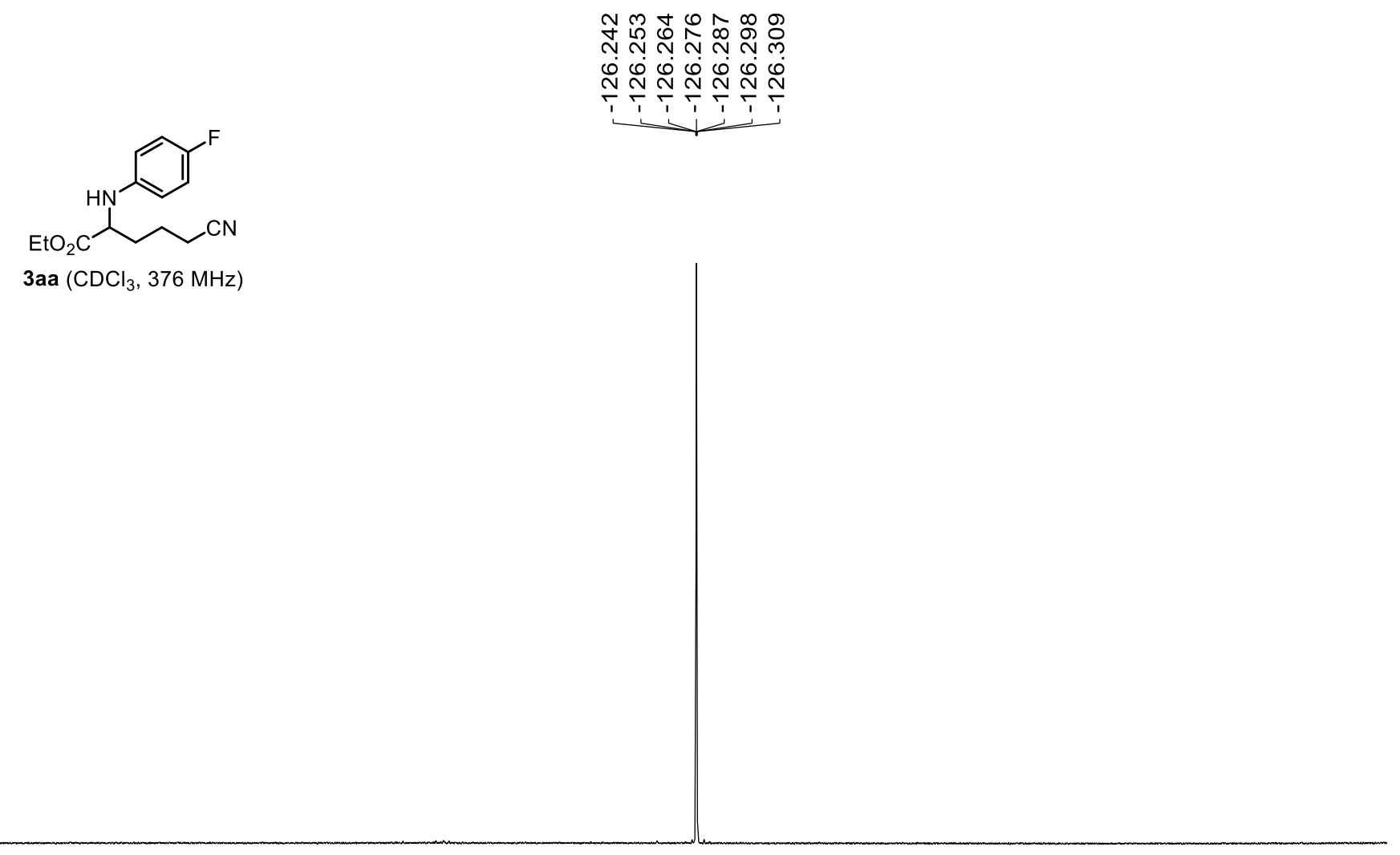

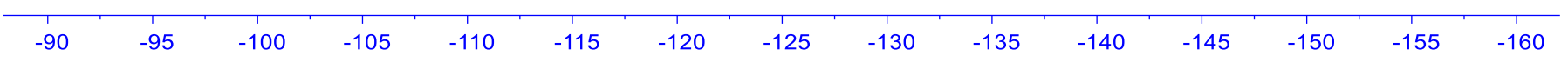




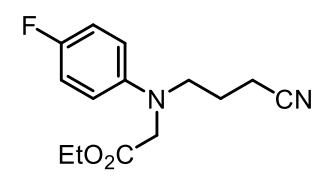

4aa $\left(\mathrm{CDCl}_{3}, 400 \mathrm{MHz}\right)$

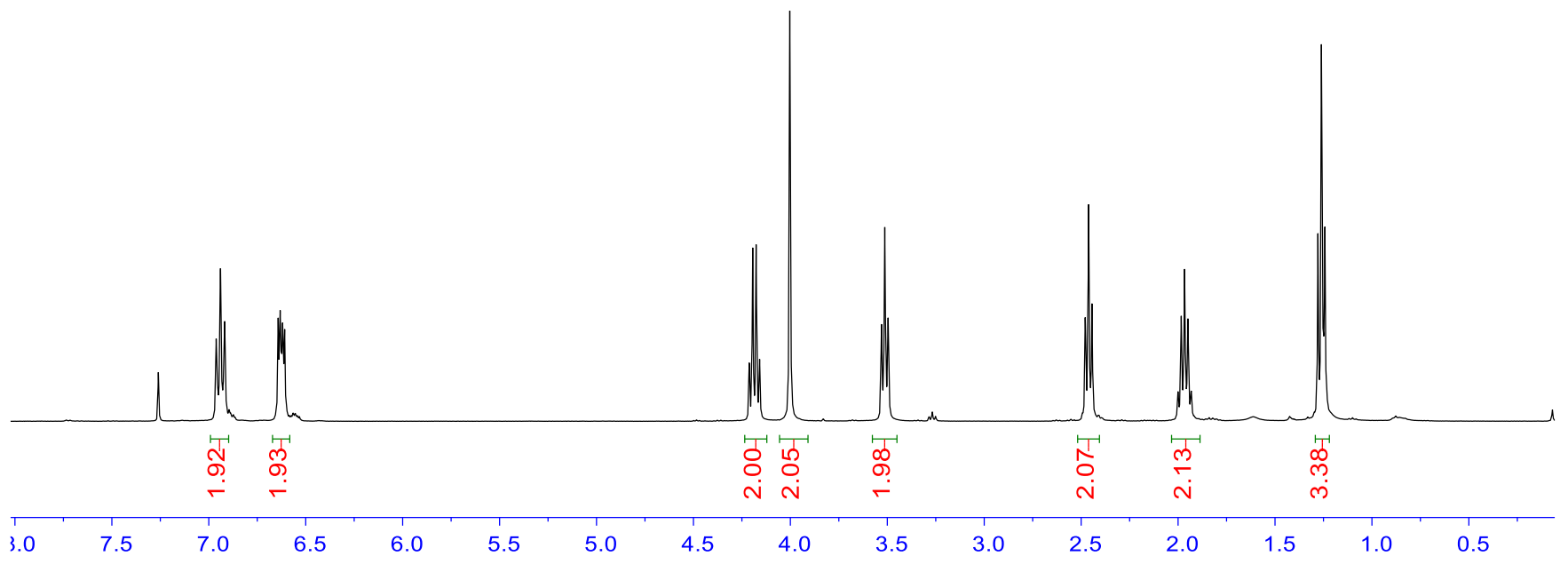

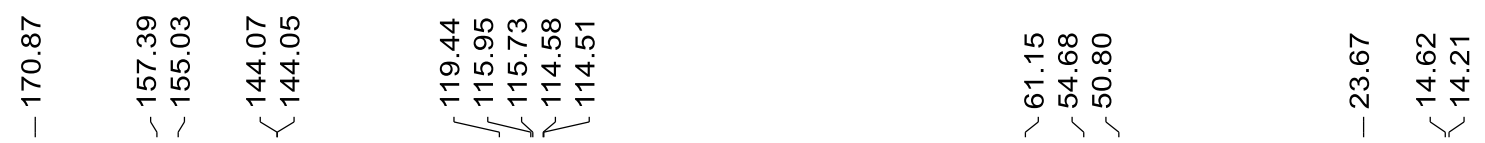<smiles>CCOC(=O)CN(CCCC#N)c1ccc(F)cc1</smiles>

4aa $\left(\mathrm{CDCl}_{3}, 101 \mathrm{MHz}\right)$ 


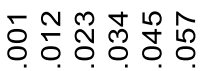

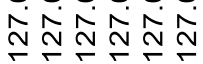

$\div \div \div \div \frac{1}{1}$

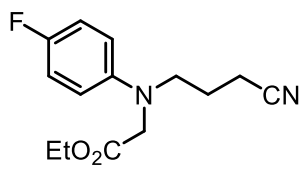

4aa $\left(\mathrm{CDCl}_{3}, 376 \mathrm{MHz}\right)$

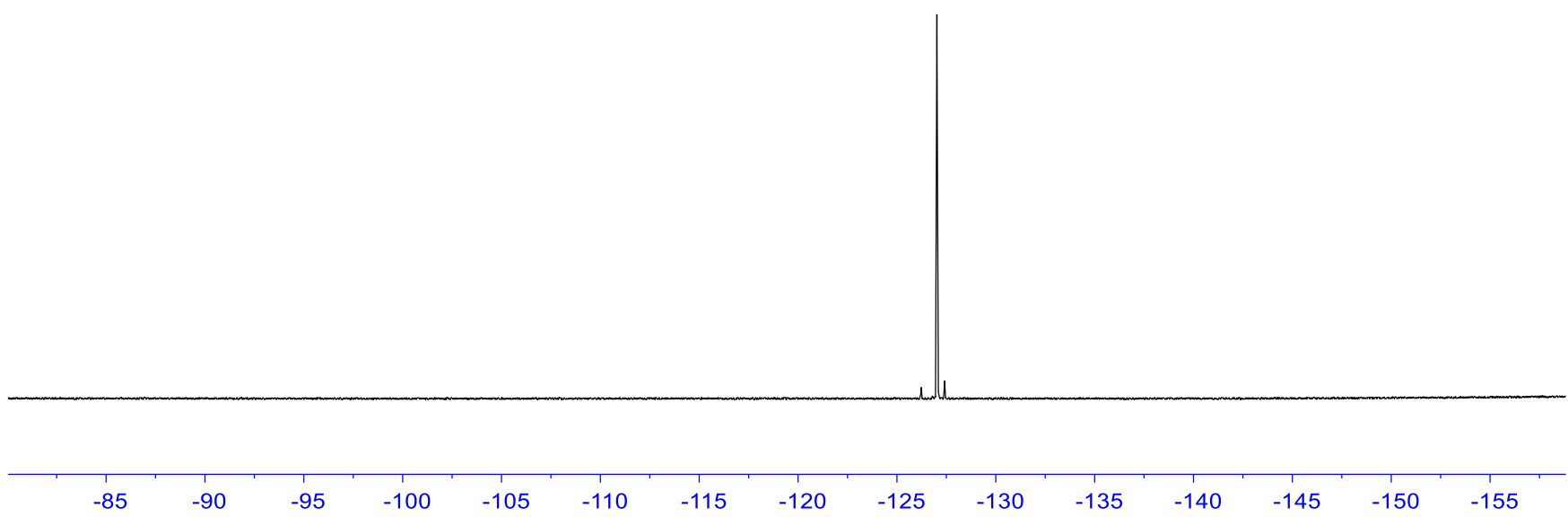



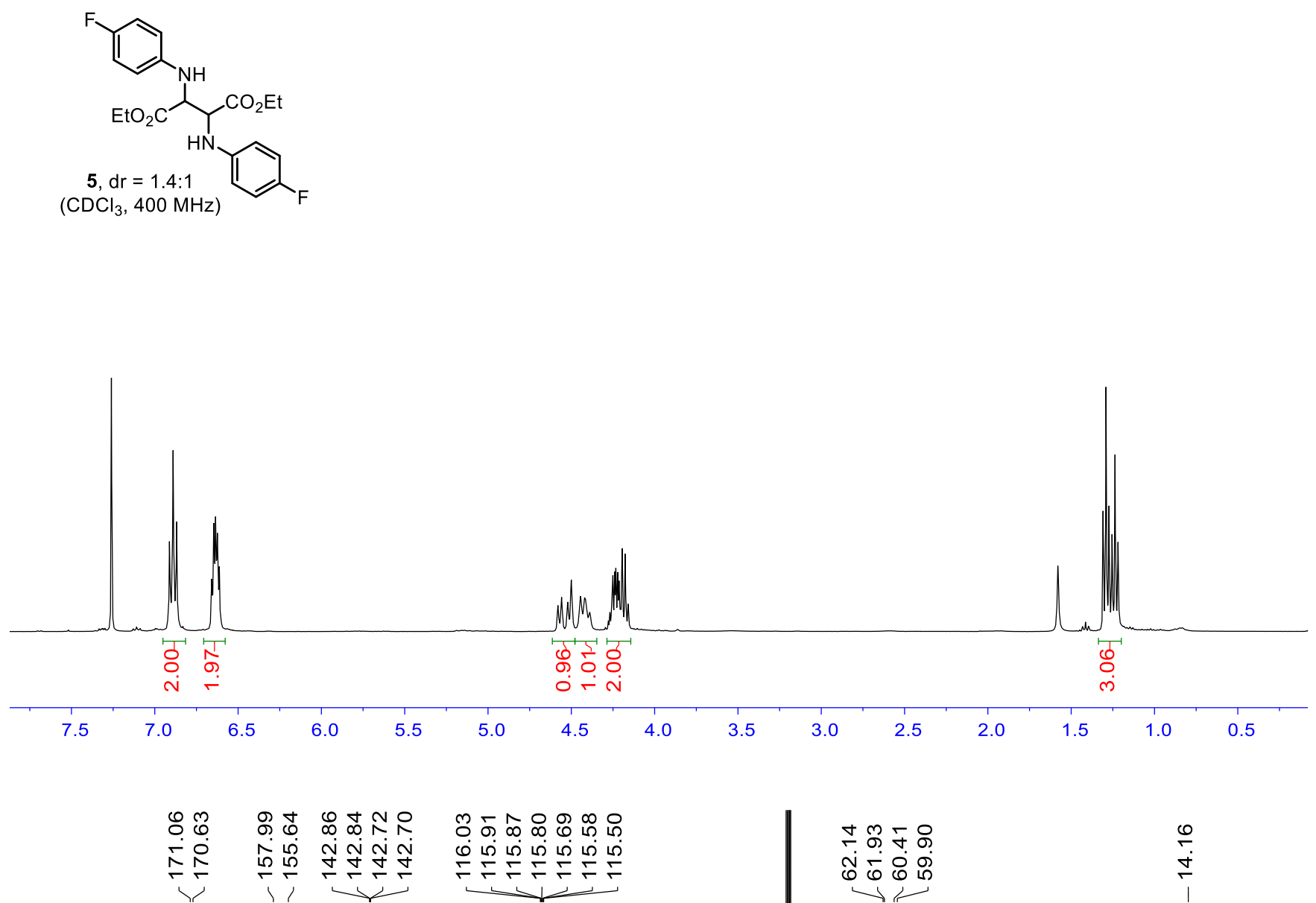

$5, \mathrm{dr}=1.4:$<smiles>CCOC(=O)C(Nc1ccc(F)cc1)C(Nc1ccc(F)cc1)C(=O)OCC</smiles>
$\left(\mathrm{CDCl}_{3}, 101 \mathrm{MHz}\right)$

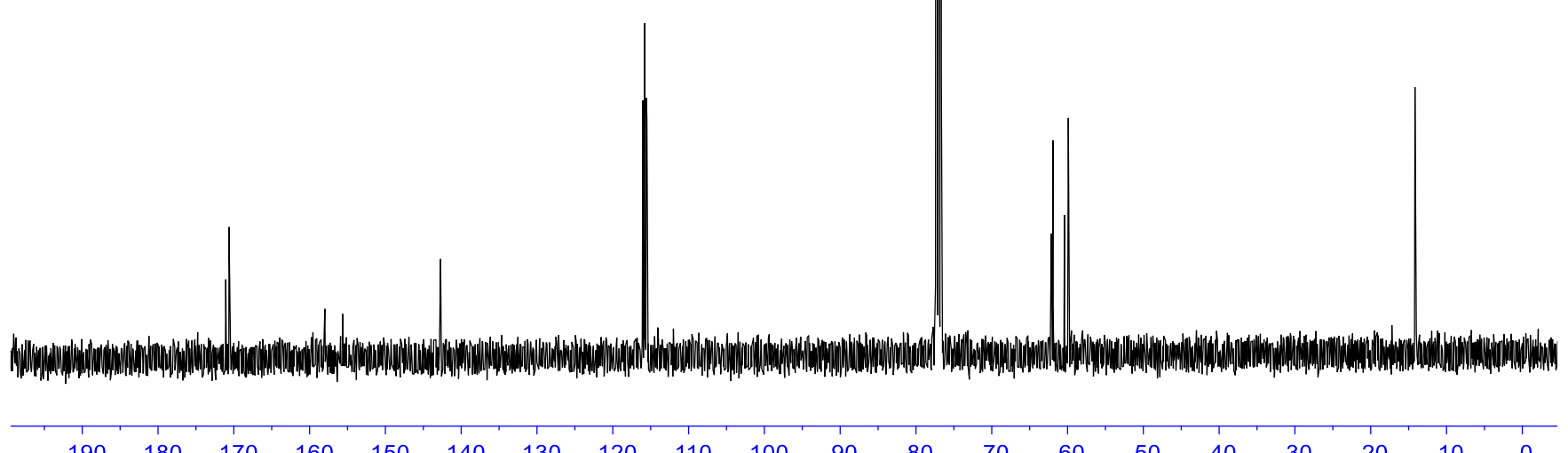




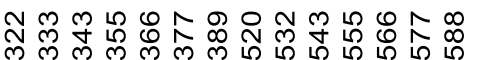

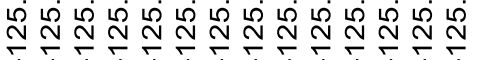

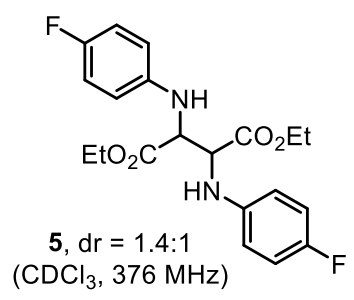

남ำ

$\circ 0$

$\begin{array}{lllllllllllllllllllllllllllll}-115 & -116 & -117 & -118 & -119 & -120 & -121 & -122 & -123 & -124 & -125 & -126 & -127 & -128 & -129 & -130 & -131 & -132 & -133 & -134 & -135 & -136 & -137 & -138 & -135\end{array}$ 
<smiles>N#CCCCC(Nc1ccc(F)cc1)C(=O)c1ccccc1</smiles>

3ba $\left(\mathrm{CDCl}_{3}, 400 \mathrm{MHz}\right)$

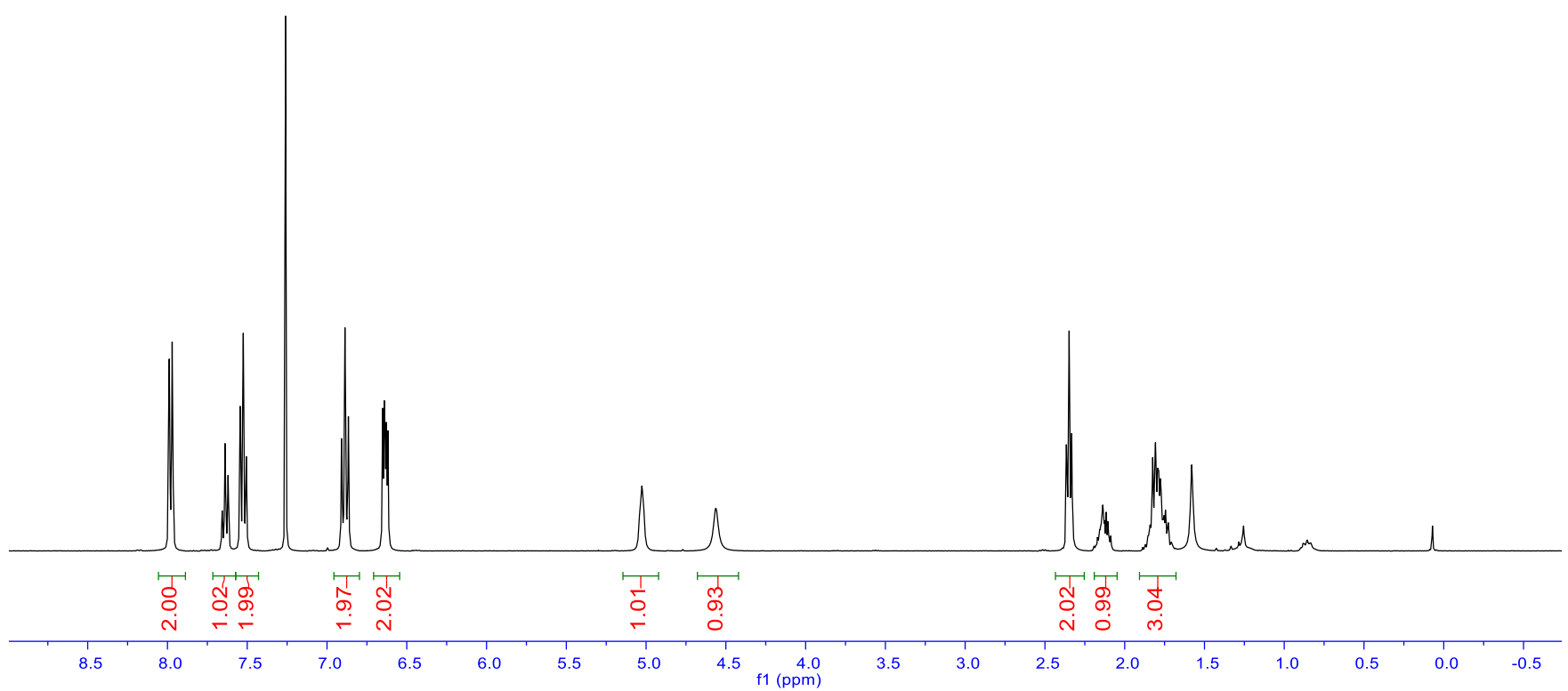

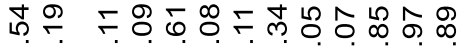

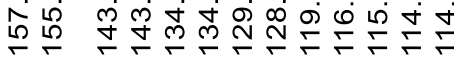<smiles>N#CCCCC(Nc1ccc(F)cc1)C(=O)c1ccccc1</smiles>

3ba $\left(\mathrm{CDCl}_{3}, 101 \mathrm{MHz}\right)$ 


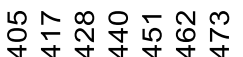

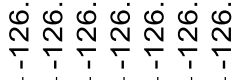

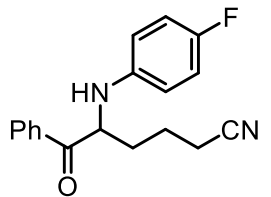

3ba $\left(\mathrm{CDCl}_{3}, 376 \mathrm{MHz}\right)$

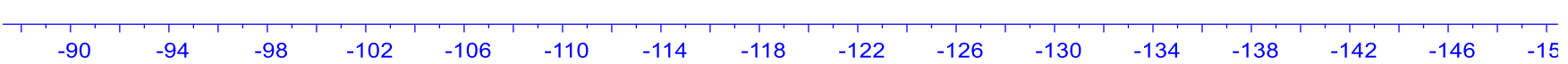


<smiles>N#CCCCC(Nc1ccc(F)cc1)C(=O)Nc1ccccc1</smiles>

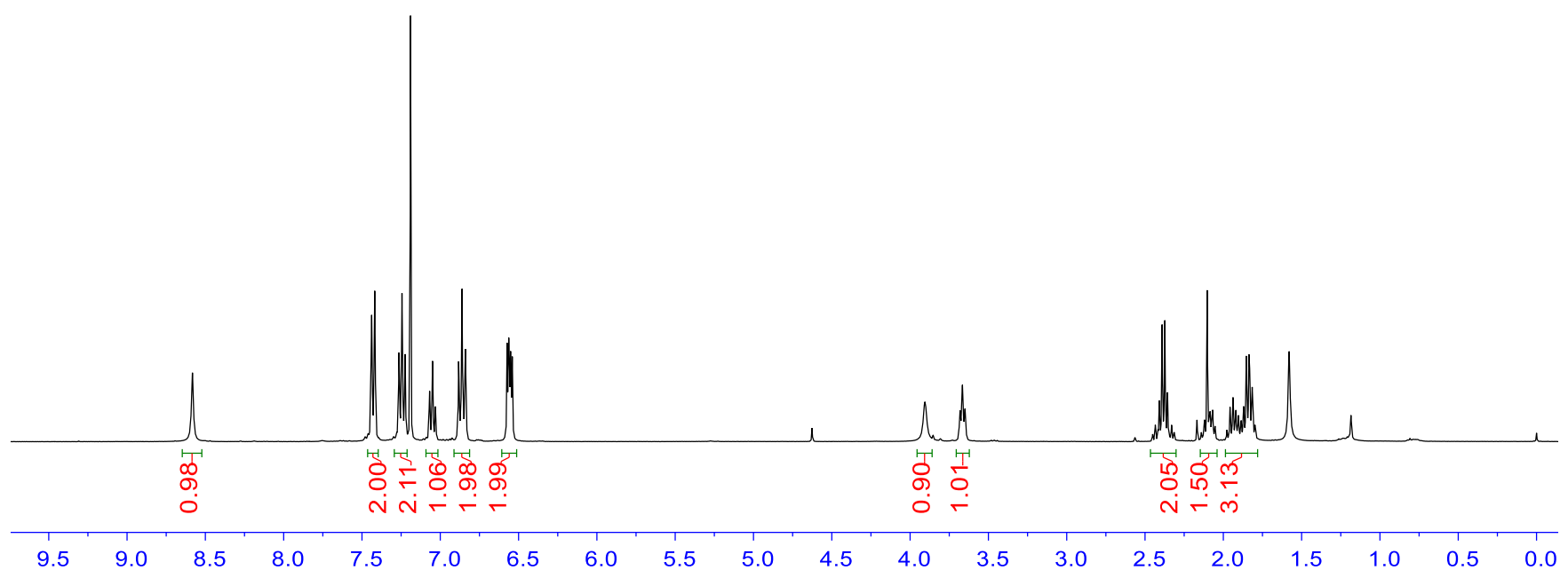<smiles>N#CCCCC(Nc1ccc(F)cc1)C(=O)Nc1ccccc1</smiles>

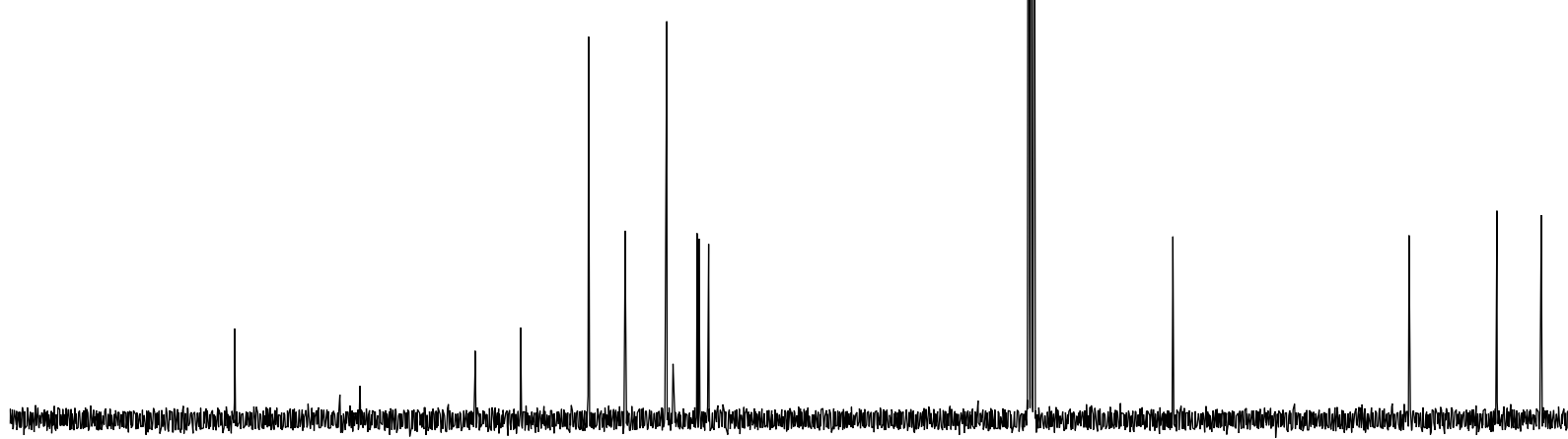


ํํำ

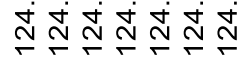

3ca $\left(\mathrm{CDCl}_{3}, 376 \mathrm{MHz}\right)$

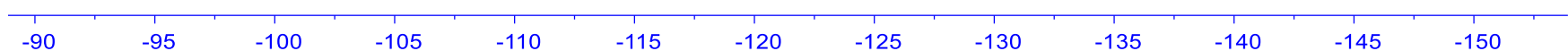




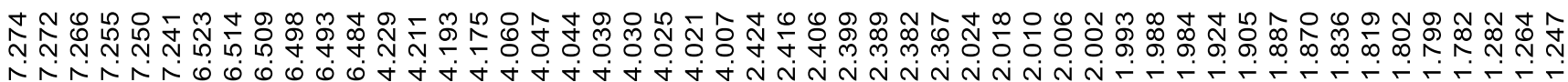<smiles>CCOC(=O)C(CCCC#N)Nc1ccc(Br)cc1</smiles>

3fa $\left(\mathrm{CDCl}_{3}, 400 \mathrm{MHz}\right)$

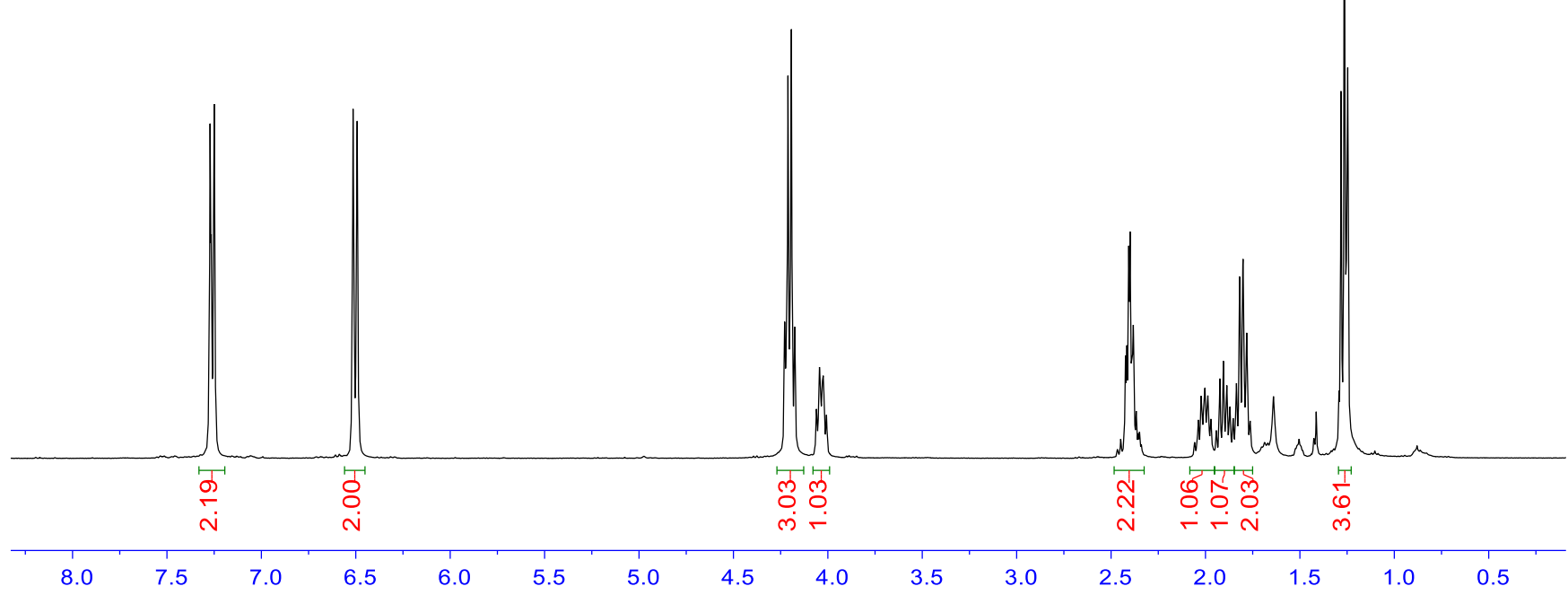

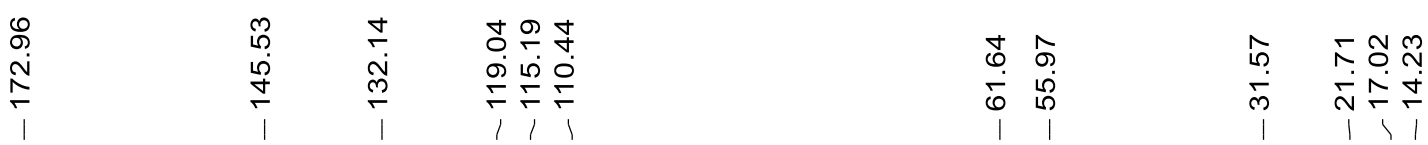

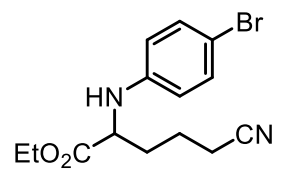

3fa $\left(\mathrm{CDCl}_{3}, 101 \mathrm{MHz}\right)$

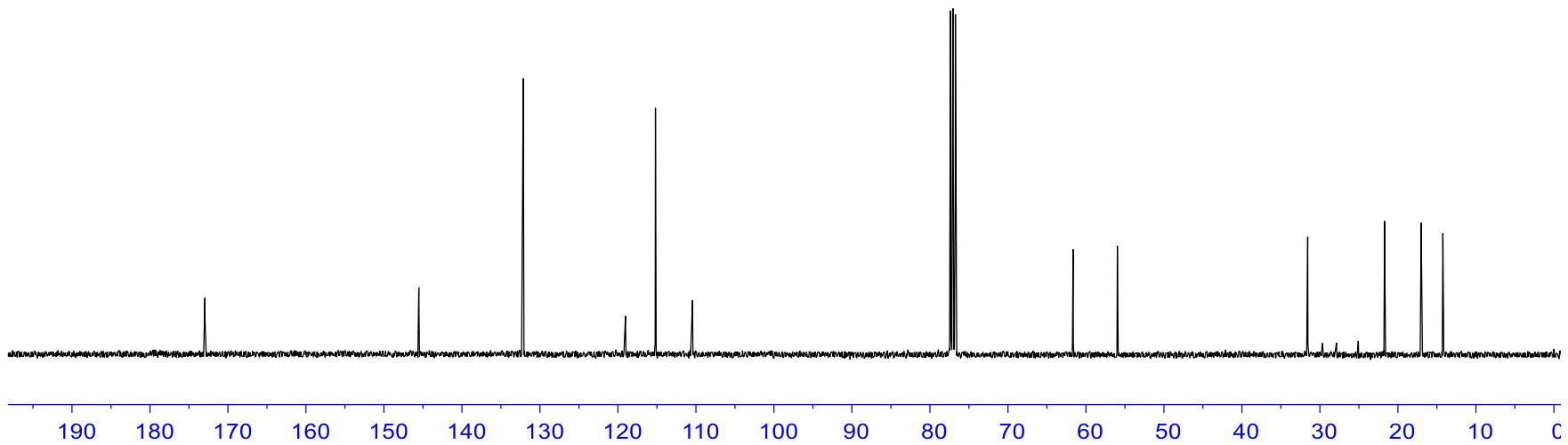




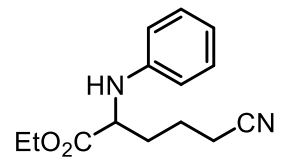

3ga $\left(\mathrm{CDCl}_{3}, 400 \mathrm{MHz}\right)$

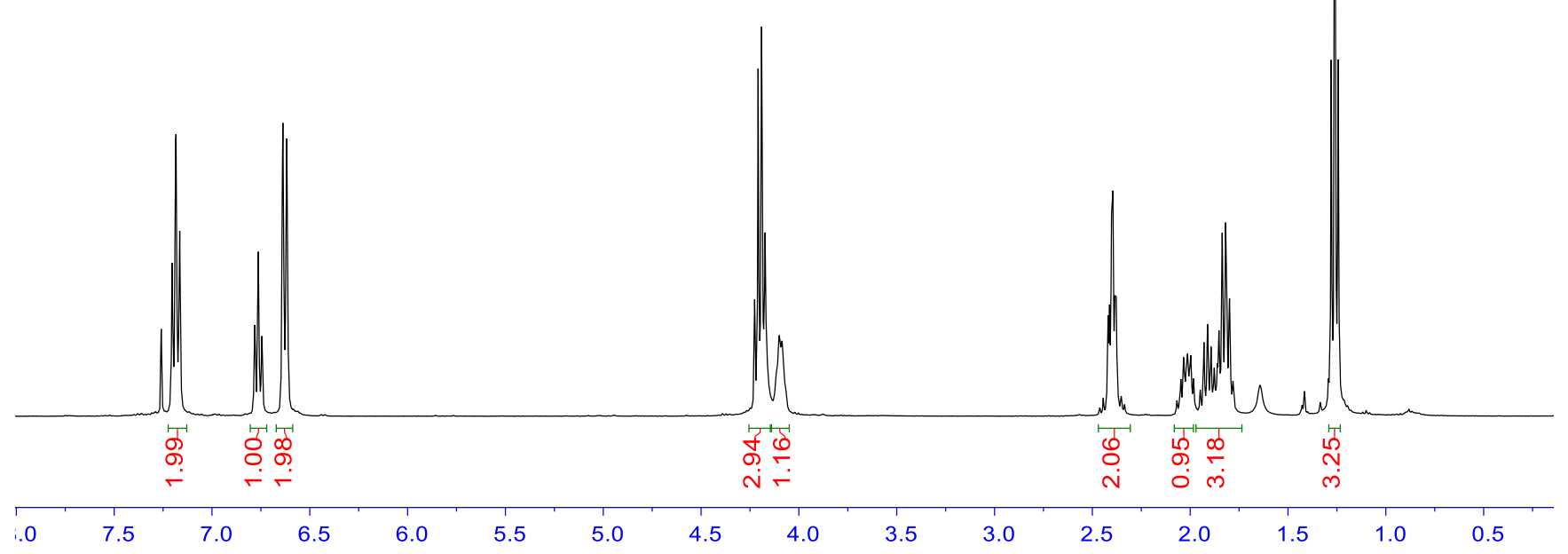

\begin{tabular}{|c|c|c|c|c|c|c|}
\hline 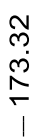 & $\begin{array}{l}5 \\
\dot{0} \\
\dot{0} \\
\dot{1} \\
1\end{array}$ & $\begin{array}{l}\stackrel{N}{+} \\
\stackrel{S}{N} \\
i\end{array}$ & 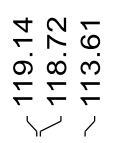 & $\begin{array}{ll}g & \hat{\sigma} \\
\dot{+} & 0 \\
\dot{\sigma} & 0 \\
1 & 1\end{array}$ & 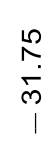 & 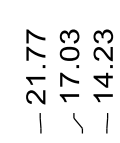 \\
\hline
\end{tabular}

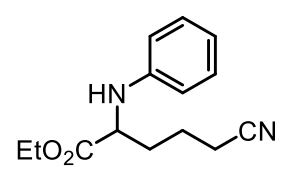

3ga $\left(\mathrm{CDCl}_{3}, 101 \mathrm{MHz}\right)$

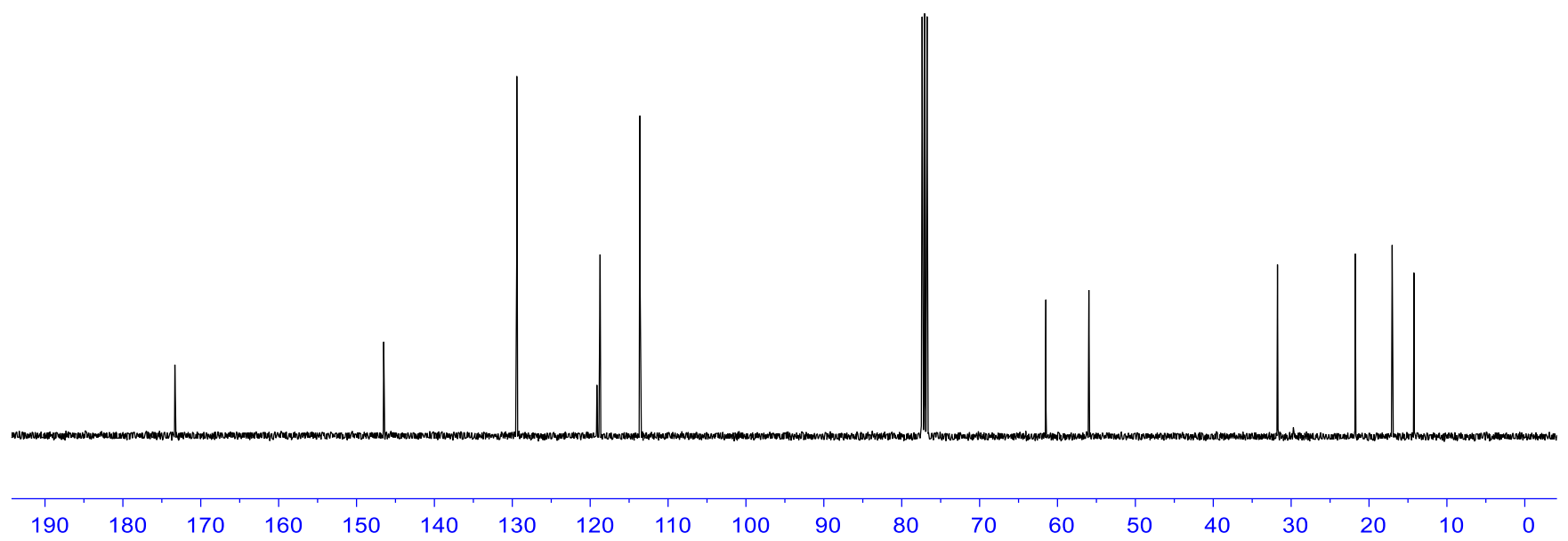




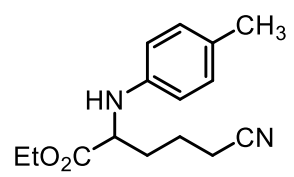

3ha $\left(\mathrm{CDCl}_{3}, 400 \mathrm{MHz}\right)$

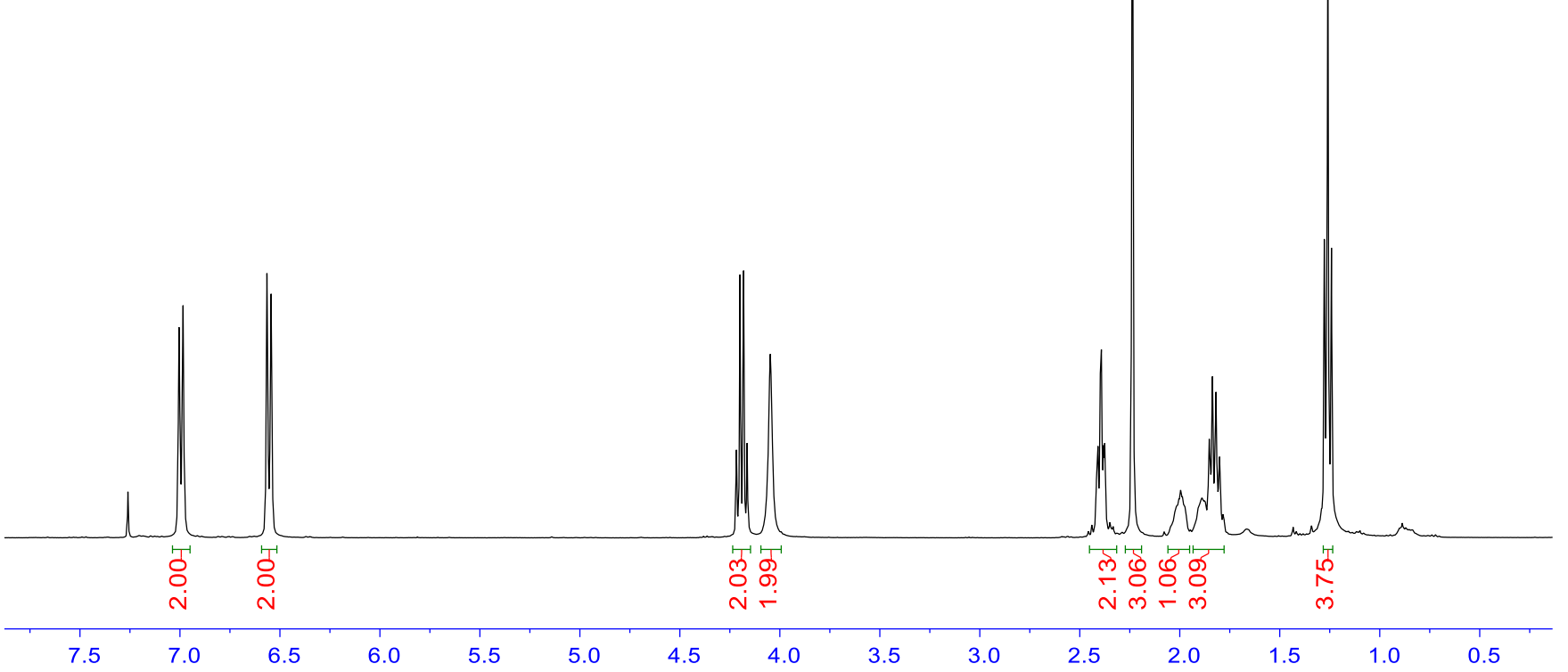

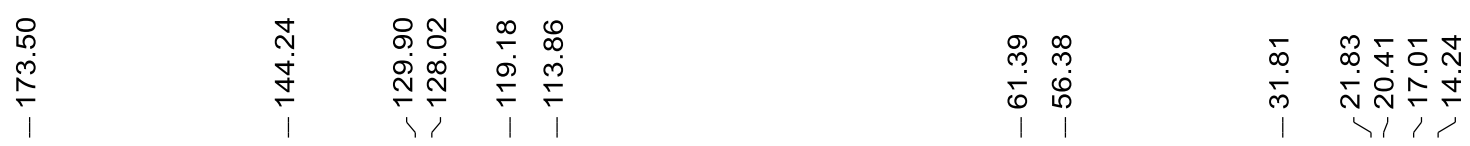

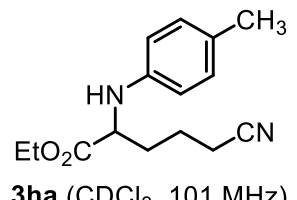

3ha $\left(\mathrm{CDCl}_{3}, 101 \mathrm{MHz}\right)$

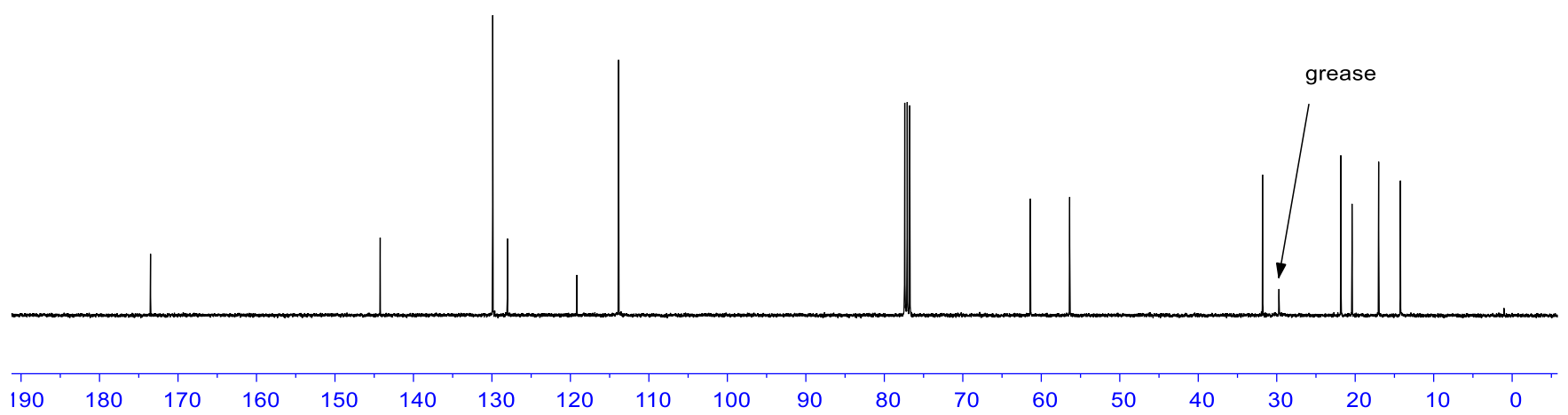




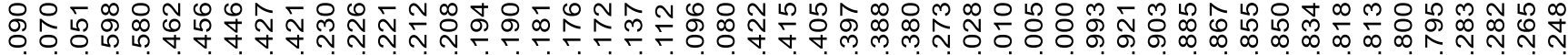

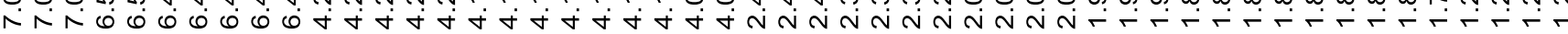<smiles>CCOC(=O)CCCCC#N</smiles>

3ia $\left(\mathrm{CDCl}_{3}, 400 \mathrm{MHz}\right)$

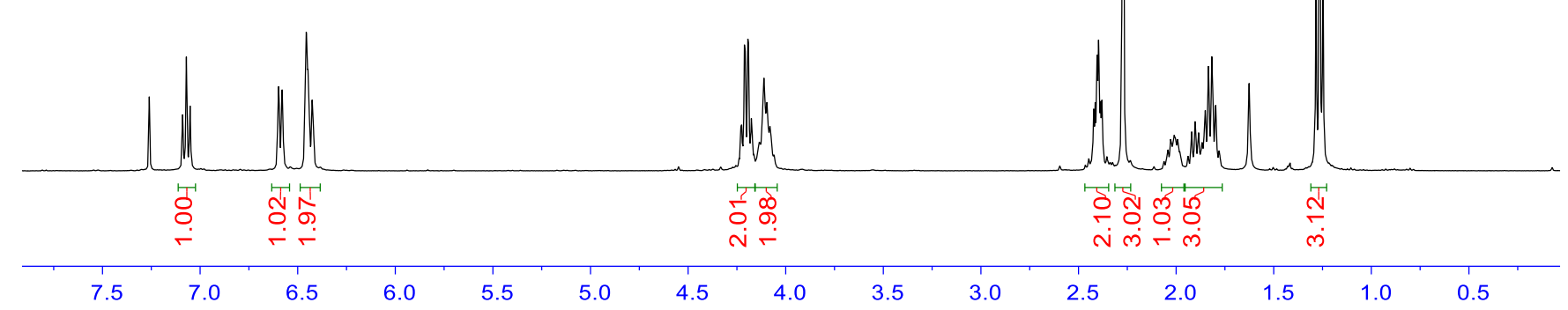

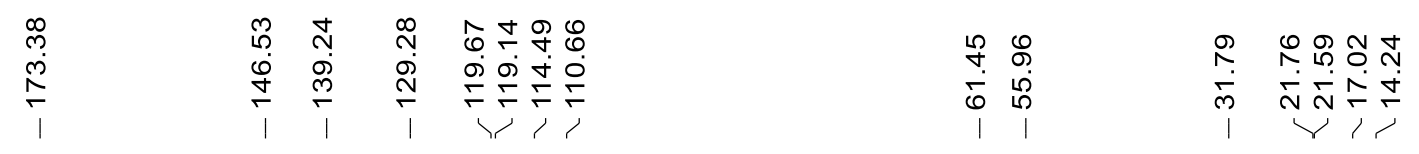

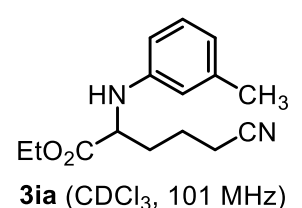

3ia $\left(\mathrm{CDCl}_{3}, 101 \mathrm{MHz}\right)$ 


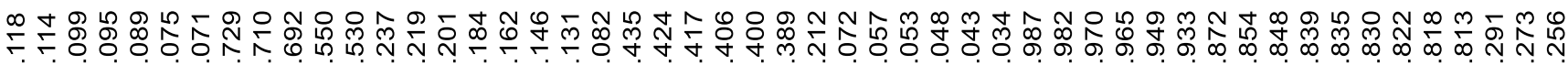

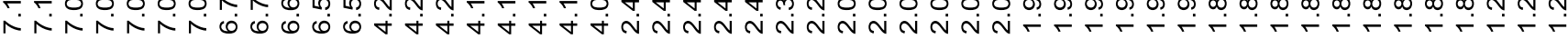<smiles>CCOC(=O)CCCCC#N</smiles>

3ja $\left(\mathrm{CDCl}_{3}, 400 \mathrm{MHz}\right)$

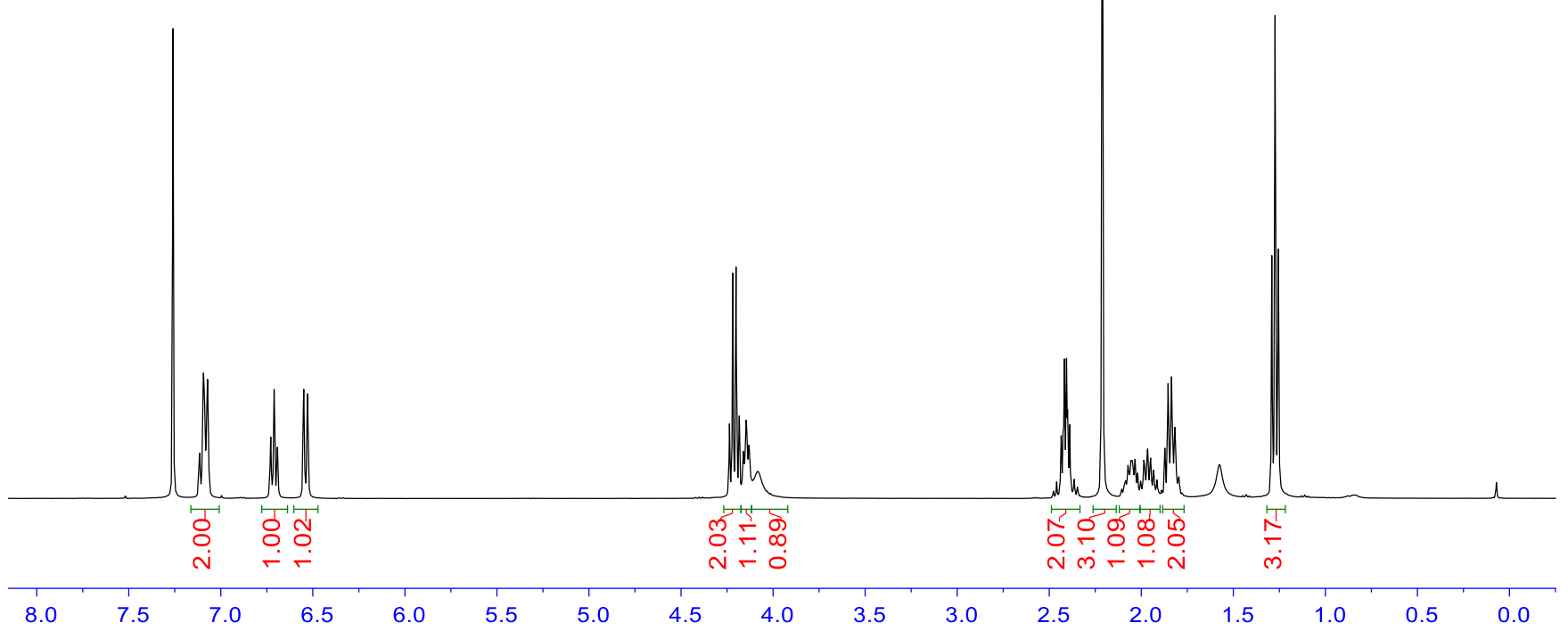

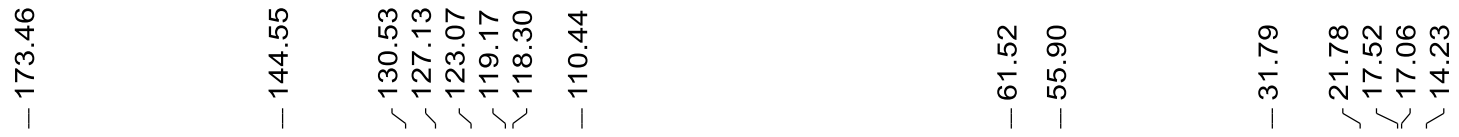

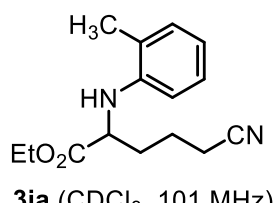

3ja $\left(\mathrm{CDCl}_{3}, 101 \mathrm{MHz}\right)$

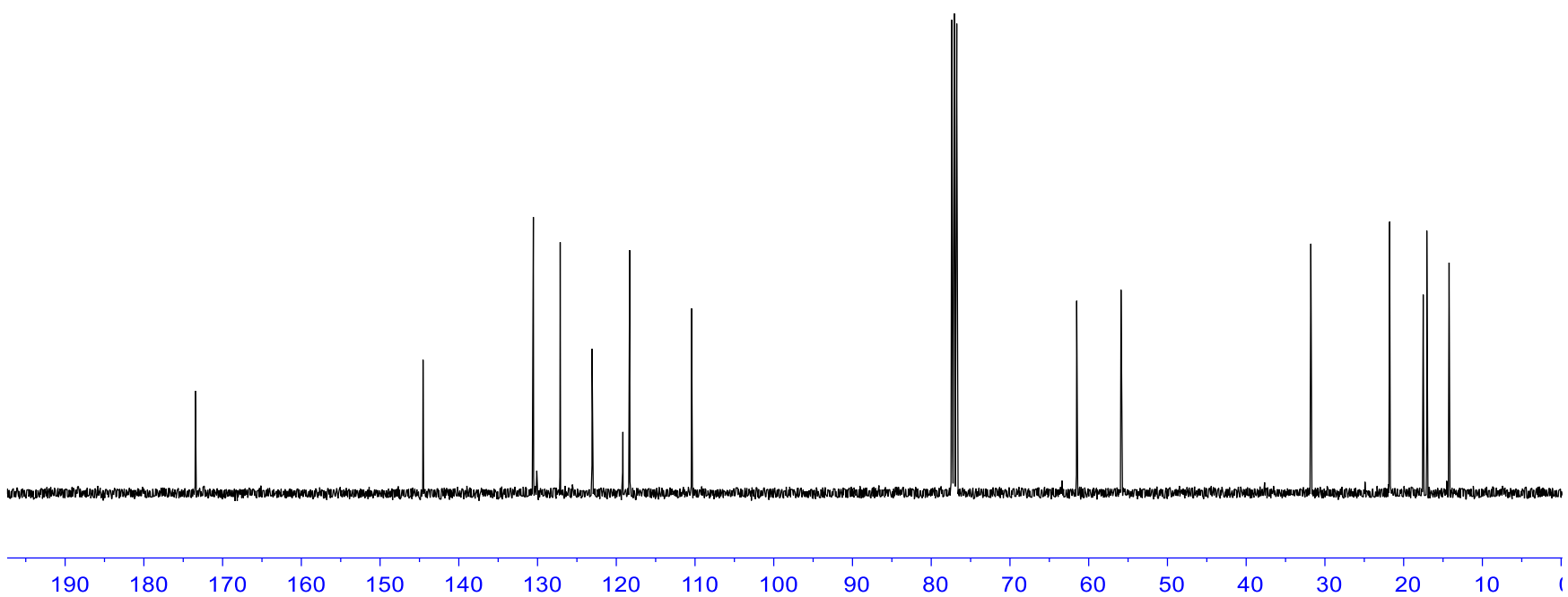




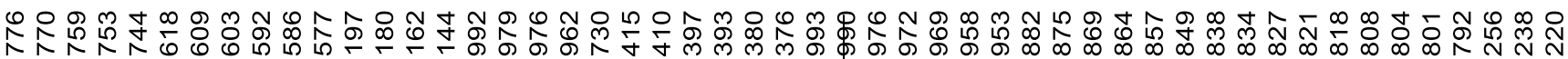

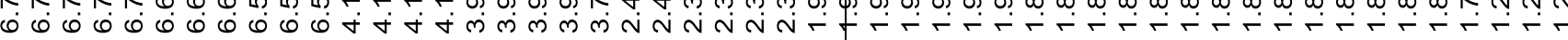<smiles>CCOC(=O)C(CCCC#N)Nc1ccc(OC)cc1</smiles>

3ka $\left(\mathrm{CDCl}_{3}, 400 \mathrm{MHz}\right)$

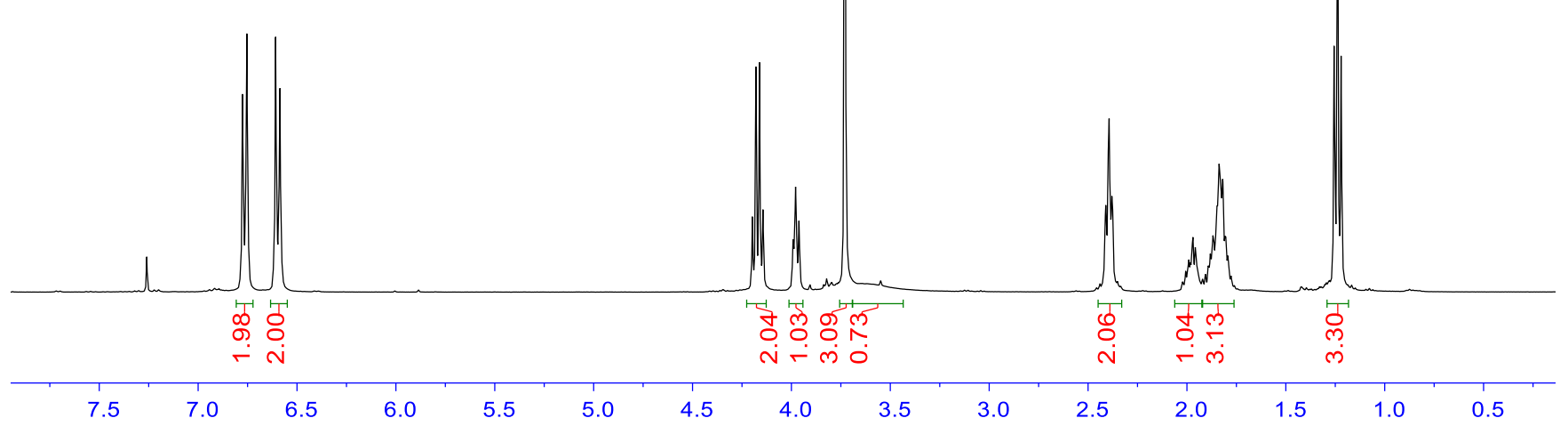

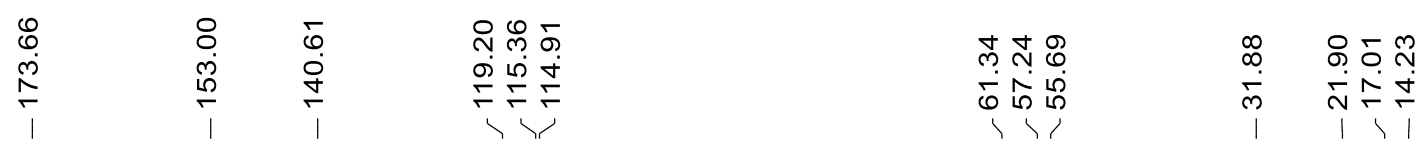

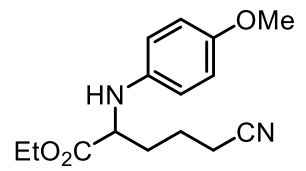

3ka $\left(\mathrm{CDCl}_{3}, 101 \mathrm{MHz}\right)$

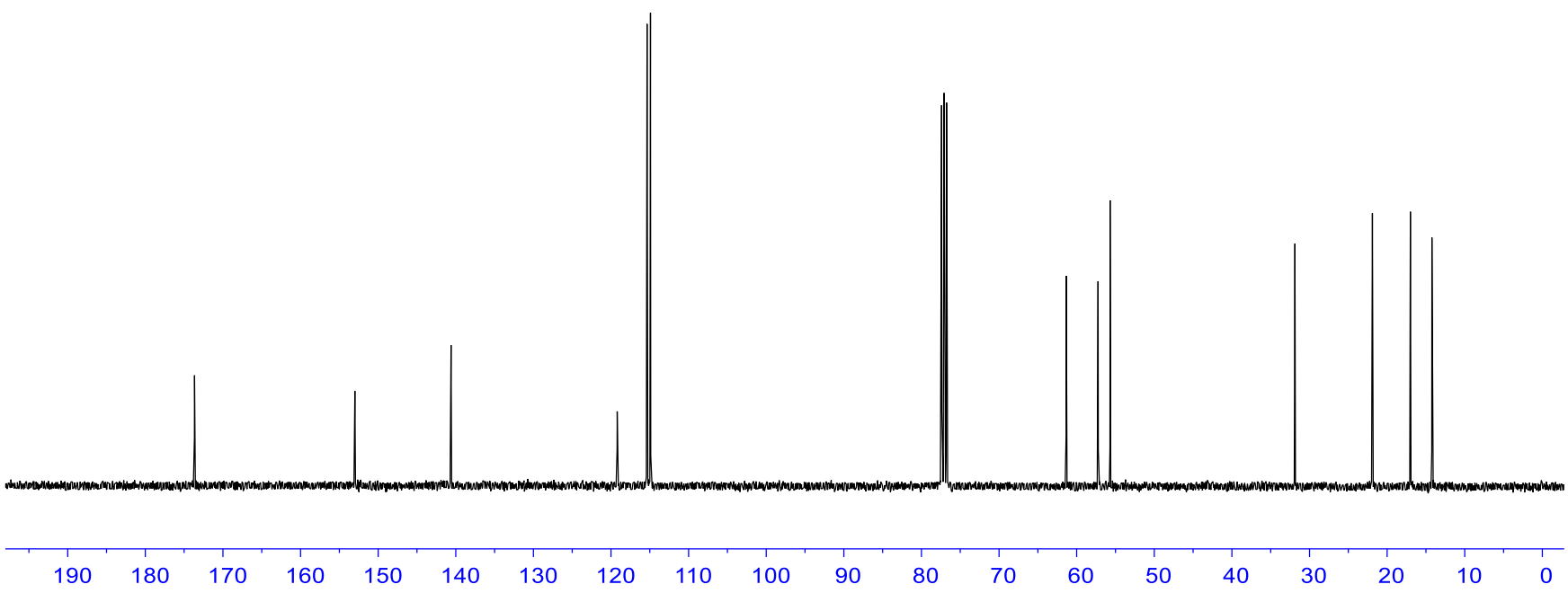




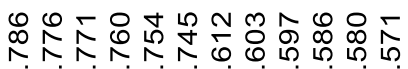

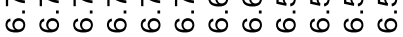<smiles>COc1ccc(NC(CCCC#N)C(=O)OC(C)(C)C)cc1</smiles>

3la $\left(\mathrm{CDCl}_{3}, 400 \mathrm{MHz}\right)$

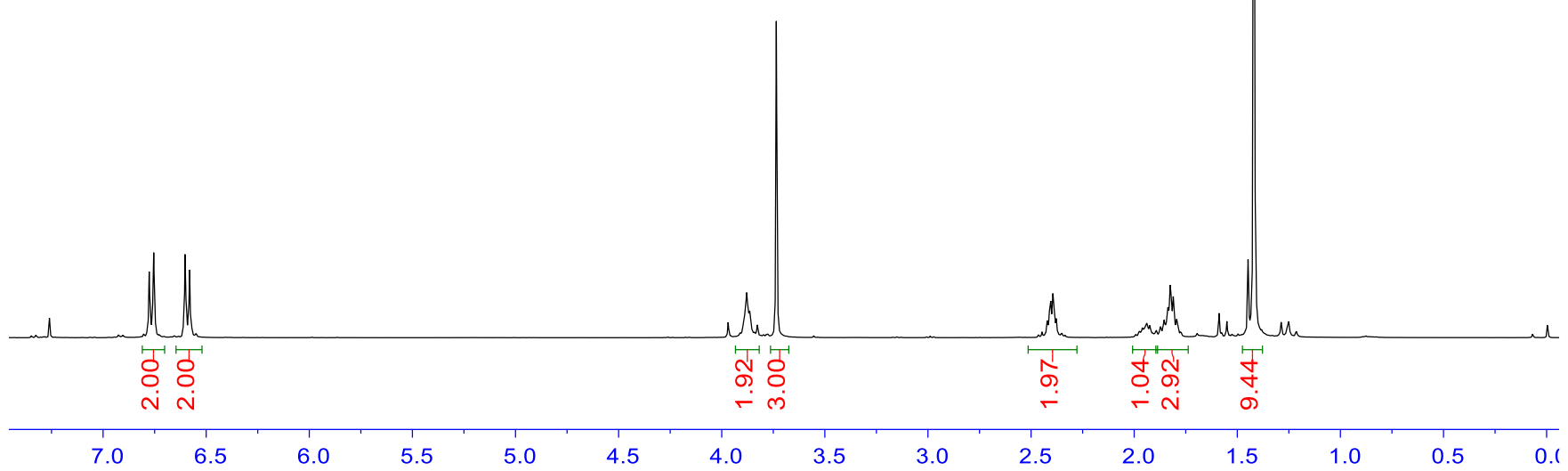

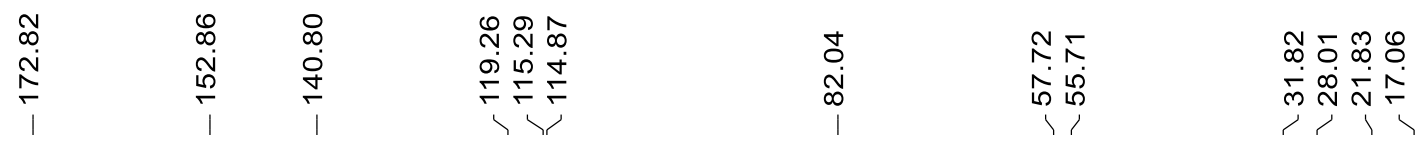

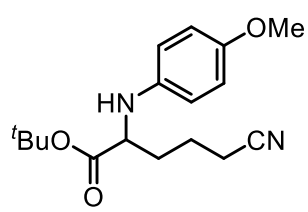

3la $\left(\mathrm{CDCl}_{3}, 101 \mathrm{MHz}\right)$

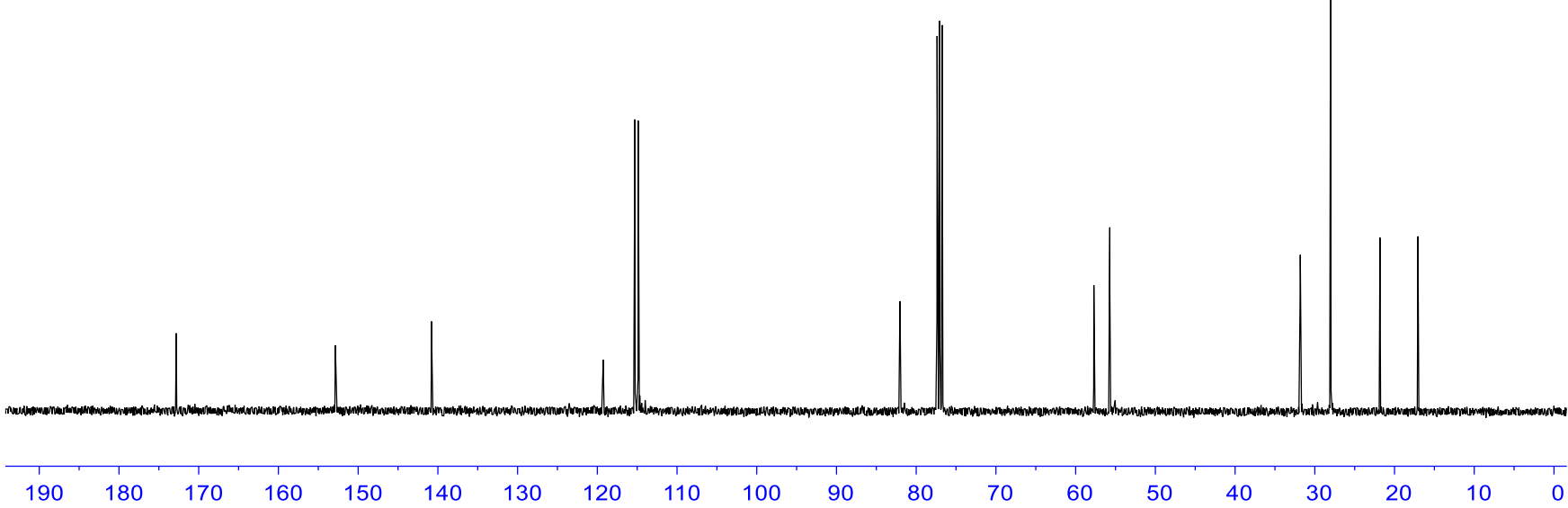




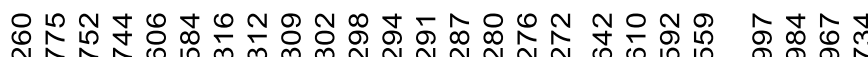

N

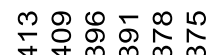<smiles>COc1ccc(NC(CCCC#N)C(=O)OCC=C(C)C)cc1</smiles>

$3 \mathrm{ma}\left(\mathrm{CDCl}_{3}, 400 \mathrm{MHz}\right)$

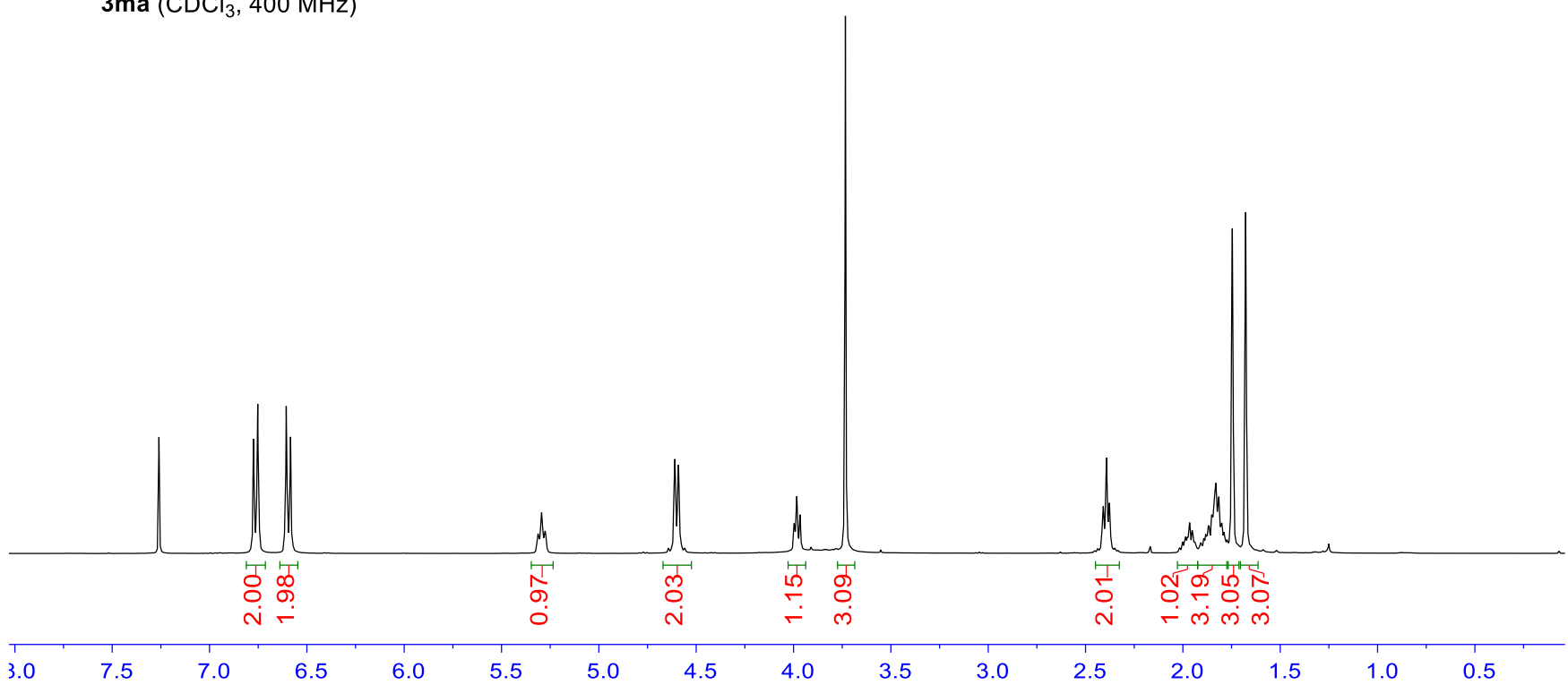

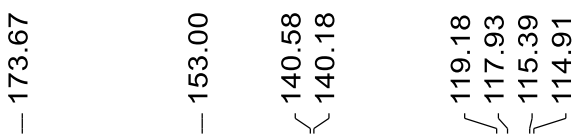

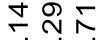

ชู เก เก

ภำㅇำ

$1<1$

m

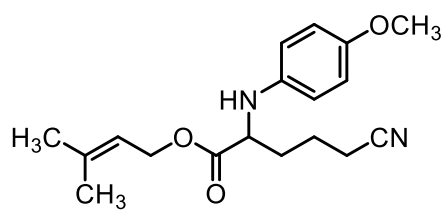

3ma $\left(\mathrm{CDCl}_{3}, 101 \mathrm{MHz}\right)$

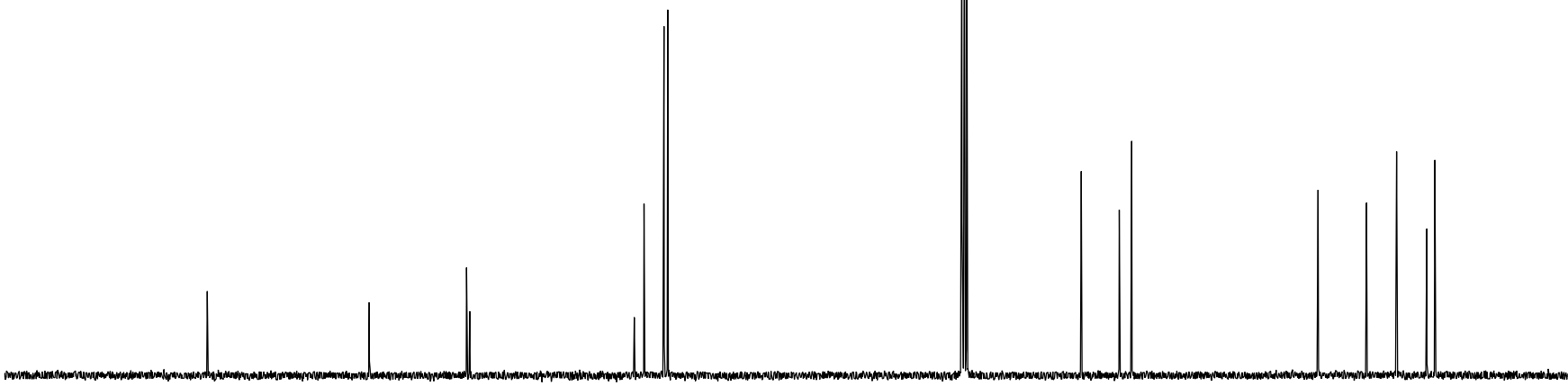


<smiles>CCCCC(Nc1ccc(OC)cc1)C(=O)c1ccccc1</smiles>
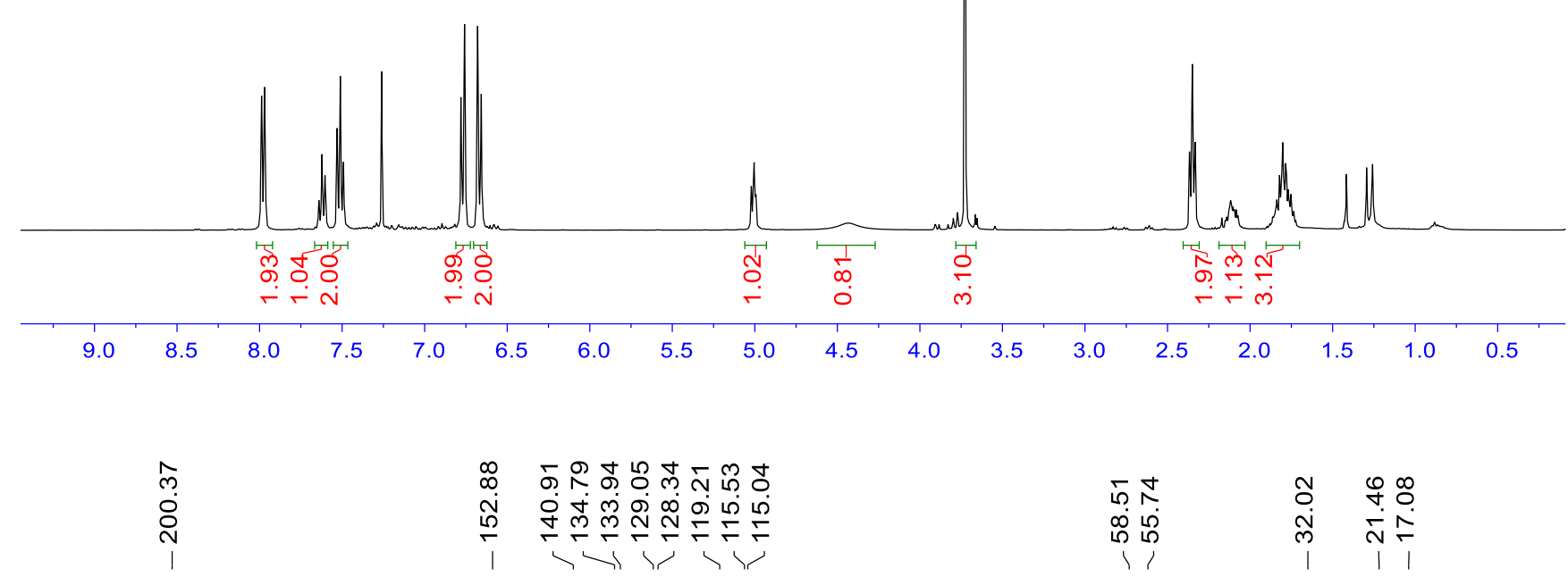

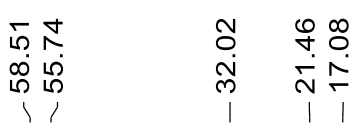

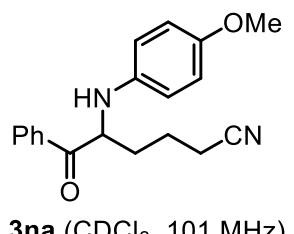

3na $\left(\mathrm{CDCl}_{3}, 101 \mathrm{MHz}\right)$

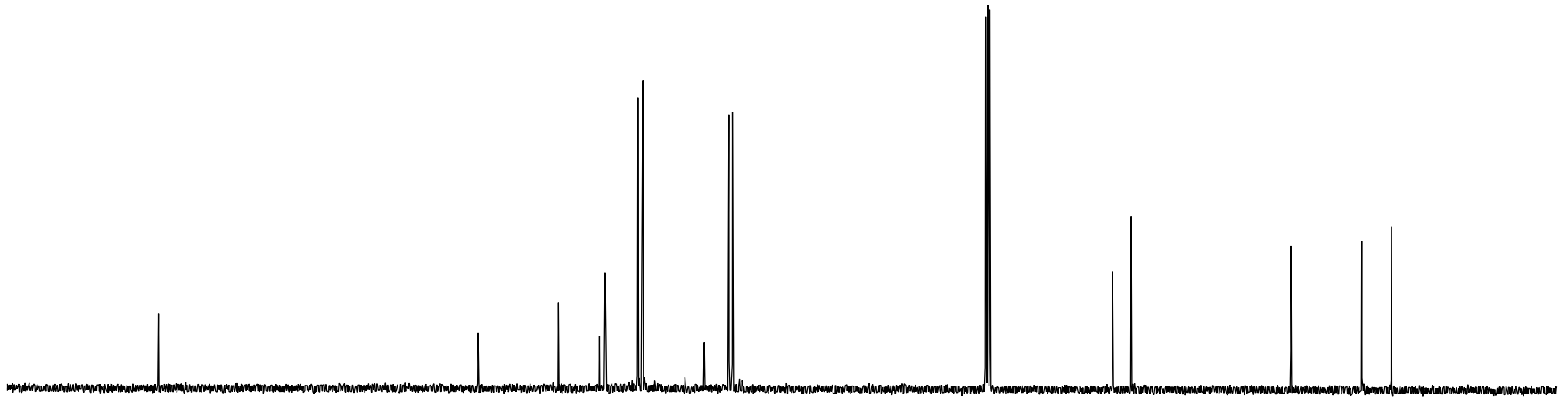

$\begin{array}{llllllllllllllllllllll}220 & 210 & 200 & 190 & 180 & 170 & 160 & 150 & 140 & 130 & 120 & 110 & 100 & 90 & 80 & 70 & 60 & 50 & 40 & 30 & 20 & 10\end{array}$ 


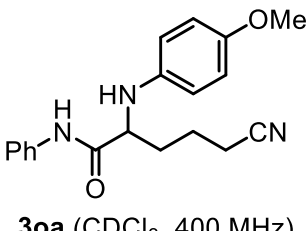

3oa $\left(\mathrm{CDCl}_{3}, 400 \mathrm{MHz}\right)$

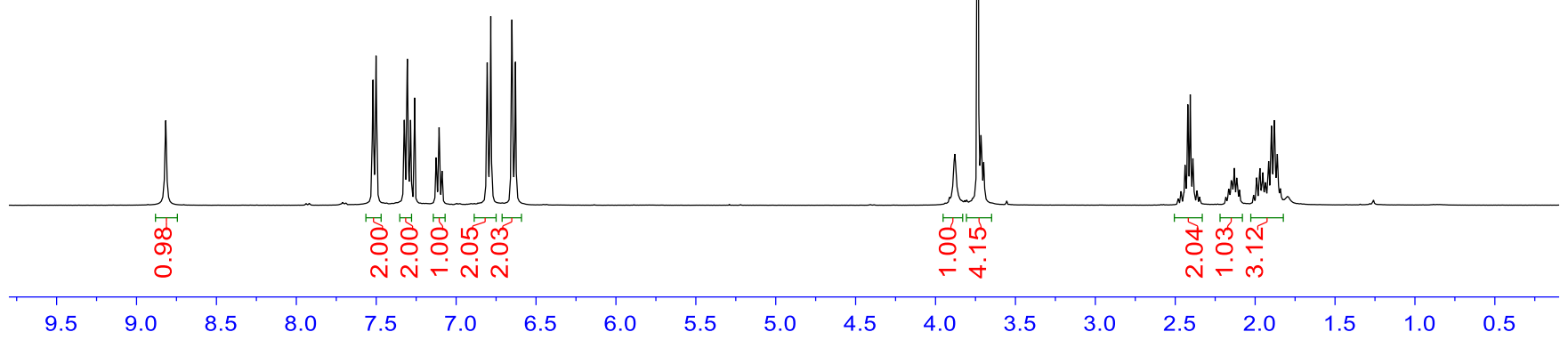

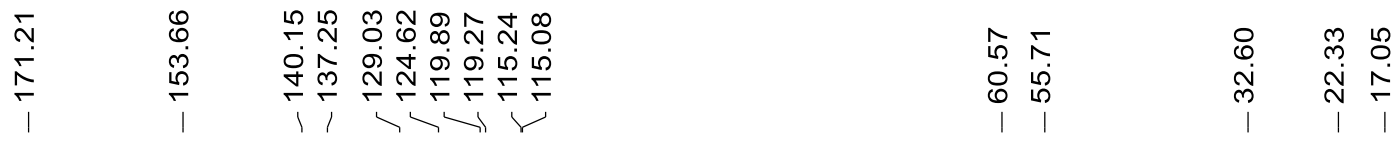<smiles>COc1ccc(NC(CCCC#N)C(=O)Nc2ccccc2)cc1</smiles>

3oa $\left(\mathrm{CDCl}_{3}, 101 \mathrm{MHz}\right)$

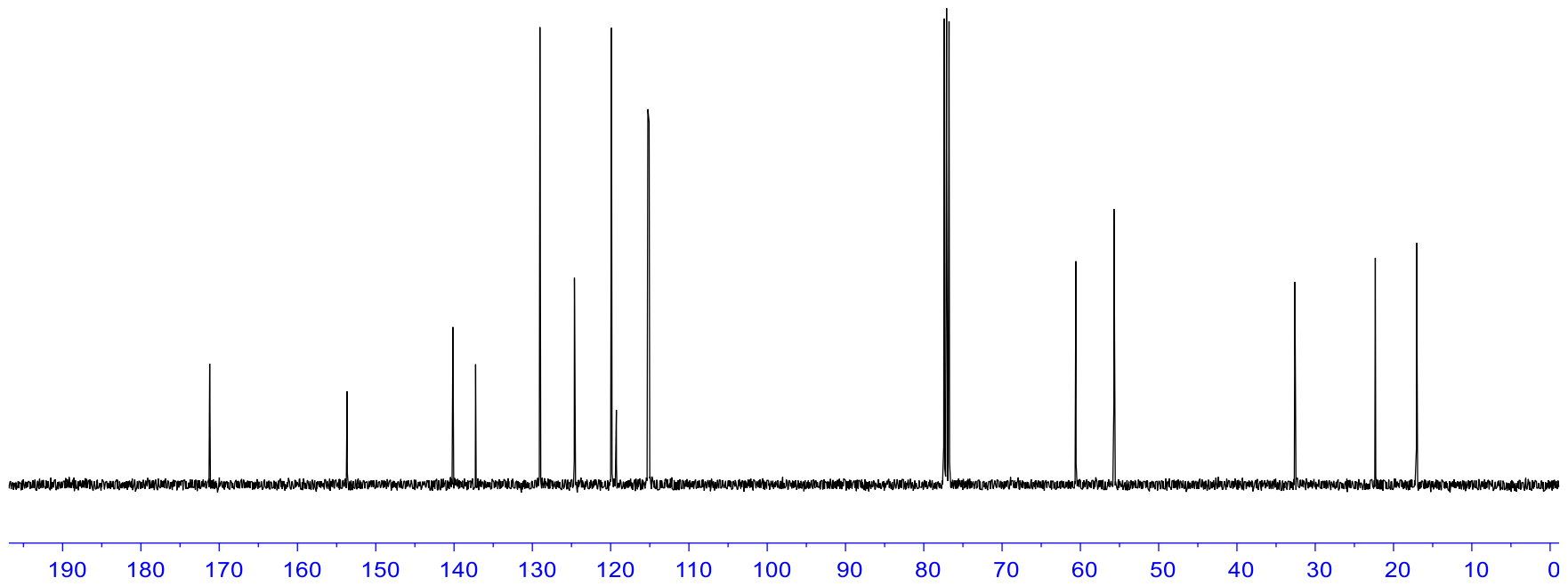




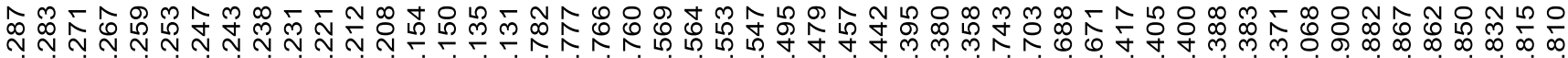

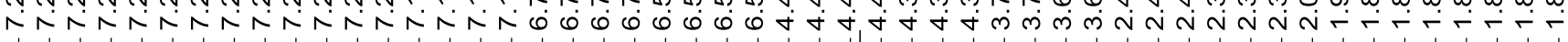<smiles>COc1ccc(NC(CCCC#N)C(=O)NCc2ccccc2)cc1</smiles>

3pa $\left(\mathrm{CDCl}_{3}, 400 \mathrm{MHz}\right)$

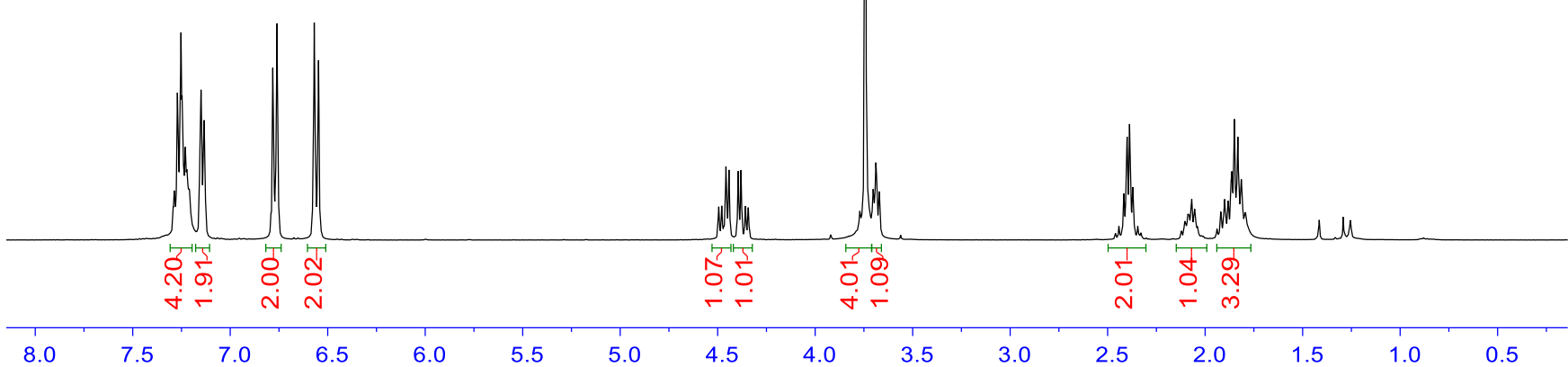

\begin{tabular}{|c|c|c|c|c|c|c|}
\hline $\begin{array}{l}\mathcal{N} \\
\infty \\
\\
\end{array}$ & $\begin{array}{c}\hat{n} \\
\text { ñ } \\
\stackrel{n}{n} \\
i\end{array}$ & 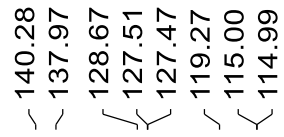 & $\begin{array}{cc}\hat{0} & 0 \\
0 & 0 \\
0 & 0 \\
1 & 10 \\
1 & 1\end{array}$ & $\stackrel{\infty}{\stackrel{\infty}{\infty}}$ & \begin{tabular}{l}
\multirow{0}{0}{} \\
ले \\
।
\end{tabular} & \\
\hline
\end{tabular}<smiles>COc1ccc(NC(CCCC#N)C(=O)NCc2ccccc2)cc1</smiles>

3 pa $\left(\mathrm{CDCl}_{3}, 101 \mathrm{MHz}\right)$

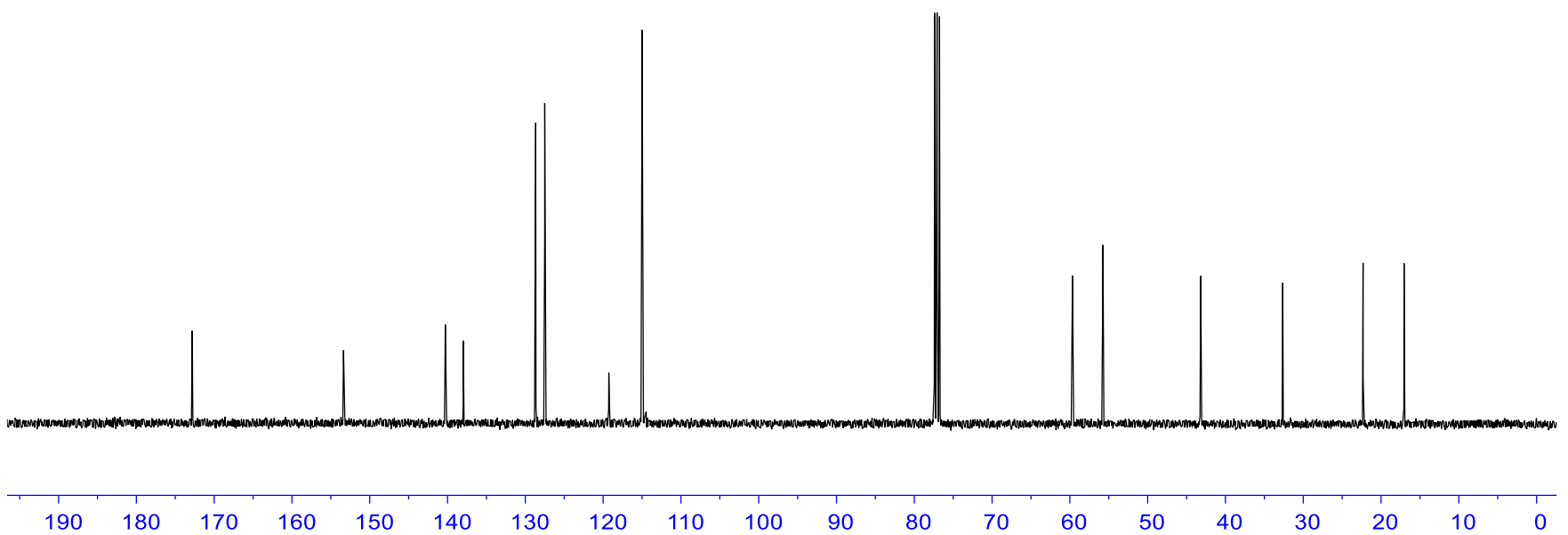


<smiles>CCOC(=O)CNC(=O)C(CCCC#N)Nc1ccc(OC)cc1</smiles>

3qa $\left(\mathrm{CDCl}_{3}, 400 \mathrm{MHz}\right)$
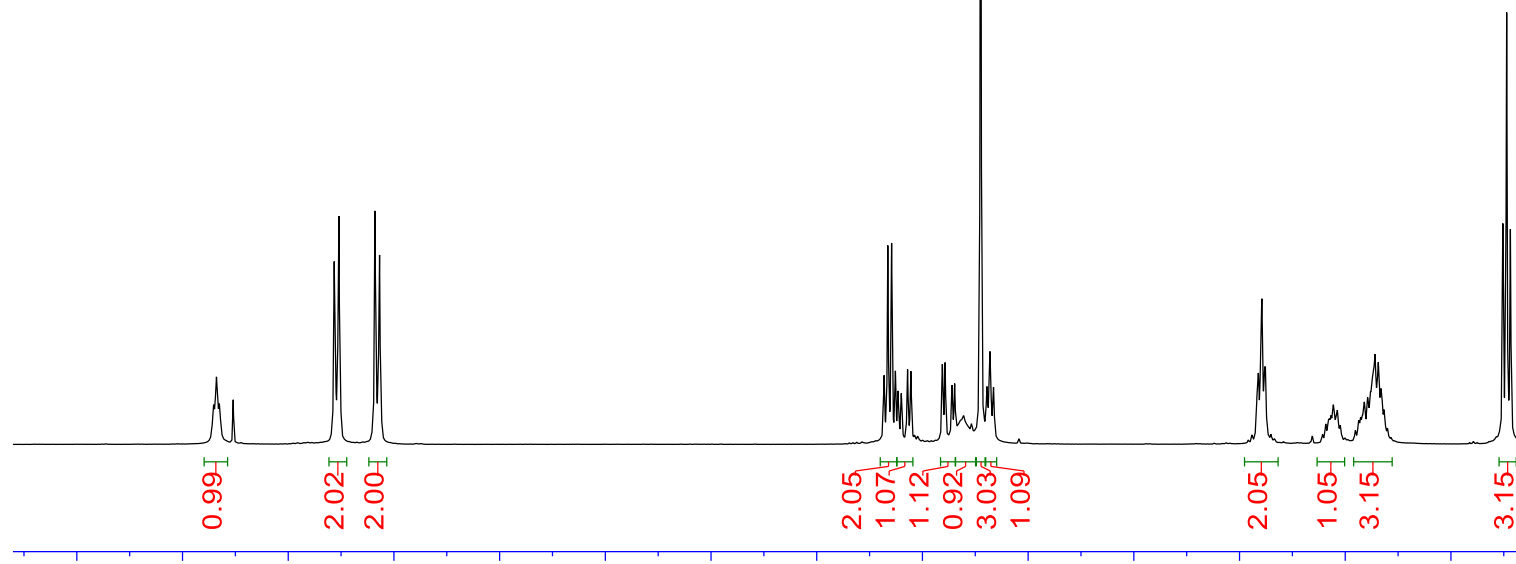

$8.0 \quad 7.5$

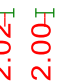

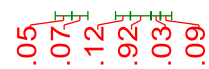

ก) ㅇํำ

号

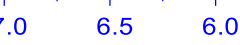

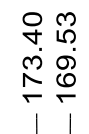

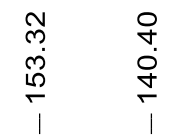

กำ ㅇํㅇ

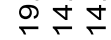

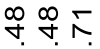

ธิ

일<smiles>CCOC(=O)CNC(=O)C(CCCC#N)Nc1ccc(OC)cc1</smiles>

3qa $\left(\mathrm{CDCl}_{3}, 101 \mathrm{MHz}\right)$

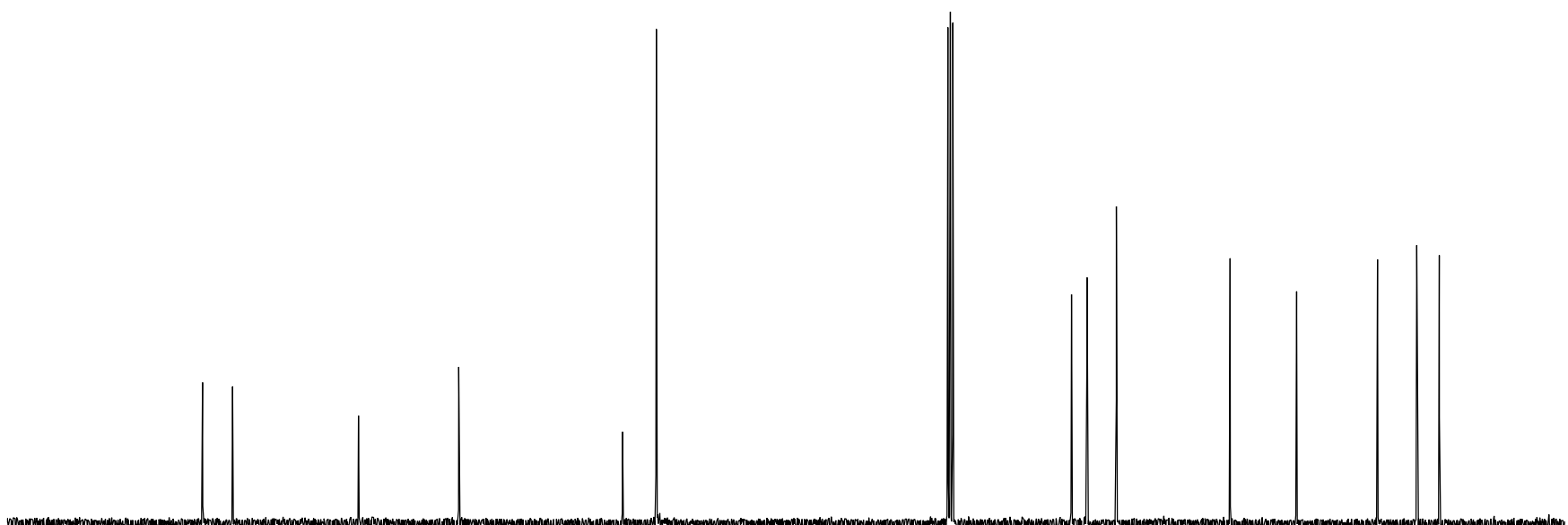


<smiles>COc1ccc(NC(CCCC#N)C(=O)N[C@@H](Cc2ccccc2)C(=O)O[Na])cc1</smiles>

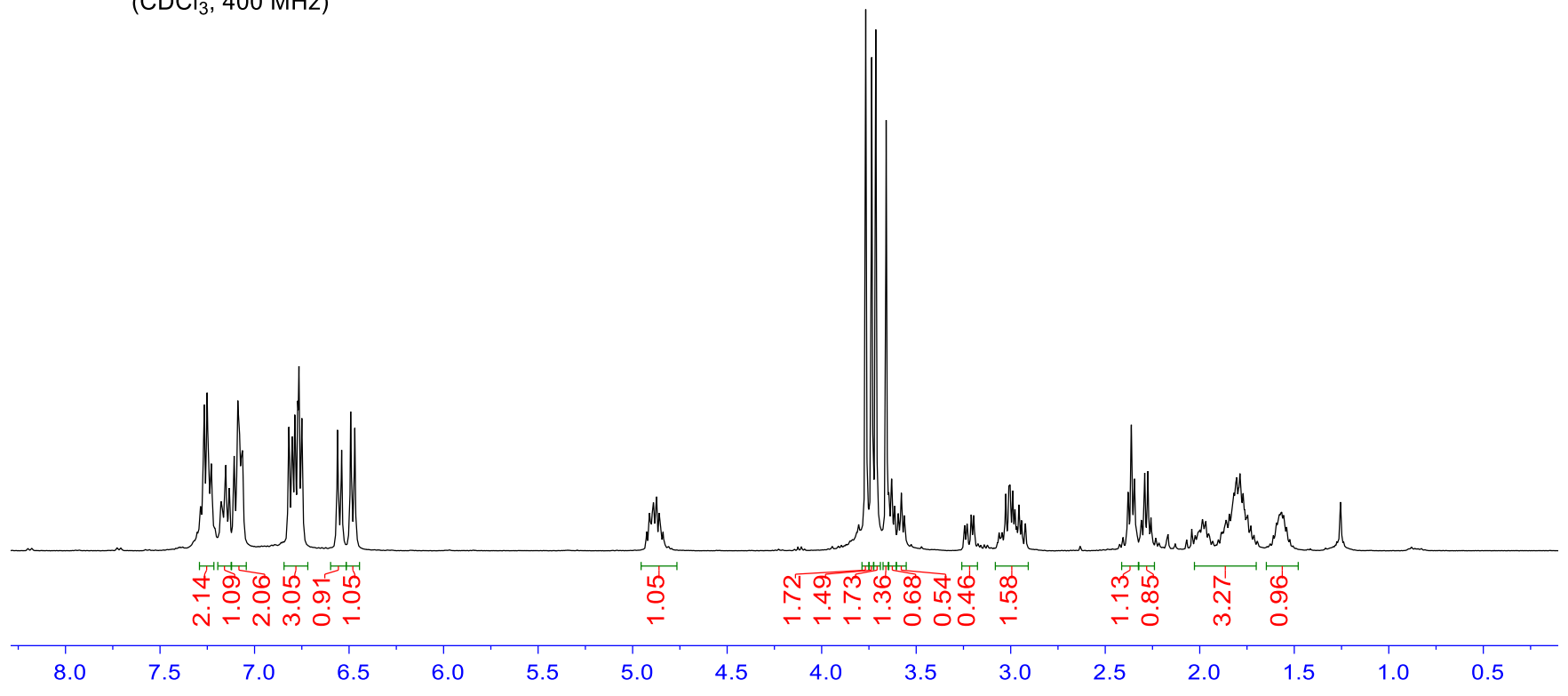

8 윤ำ

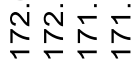
눈 岗<smiles>COc1ccc(NC(CCCC#N)C(=O)N[C@@H](Cc2ccccc2)C(C)=O)cc1</smiles>

3ra, $d r=1.2: 1$

$\left(\mathrm{CDCl}_{3}, 101 \mathrm{MHz}\right)$

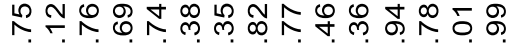
คํํำ เึ่ ถึ่

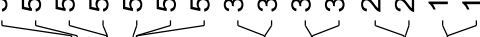

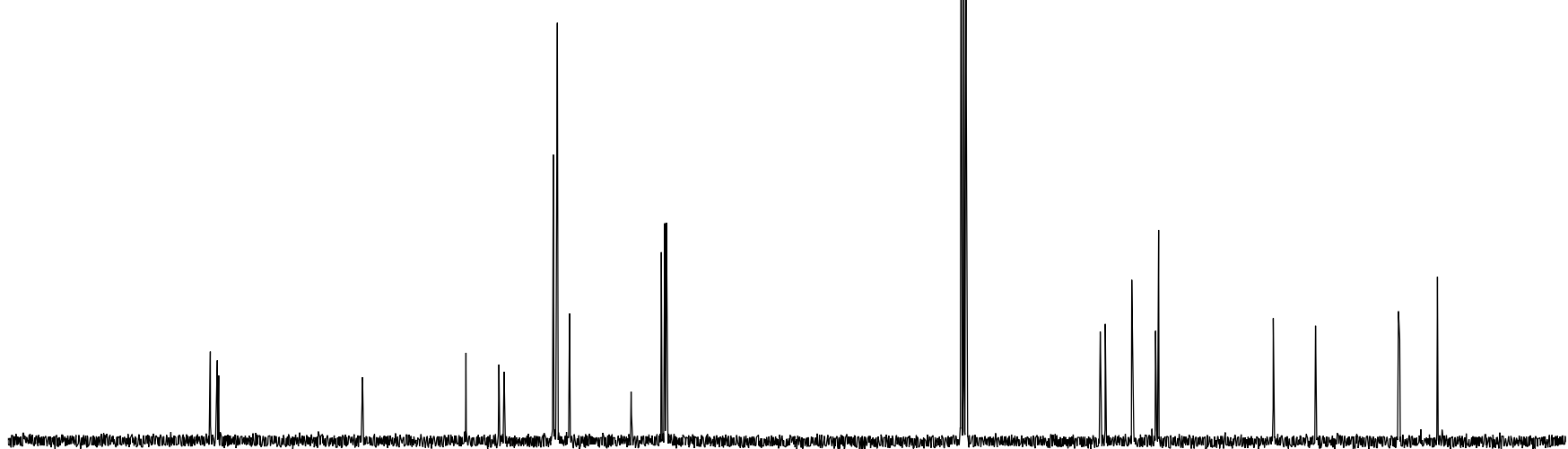




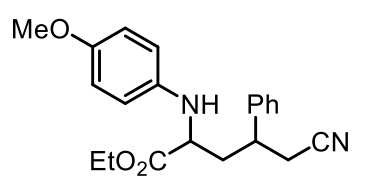

$\mathbf{3 k b}, d r=1.3: 1$

$\left(\mathrm{CDCl}_{3}, 400 \mathrm{MHz}\right)$

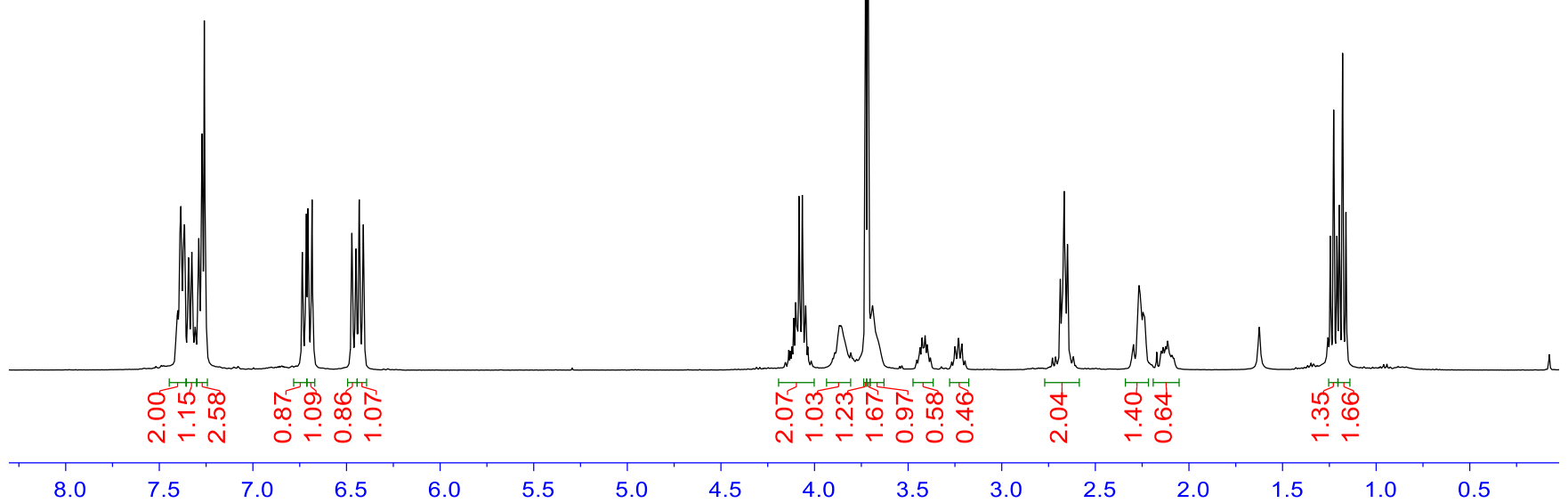

क人

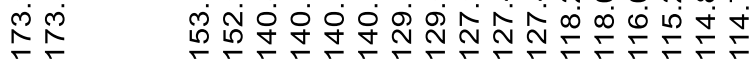
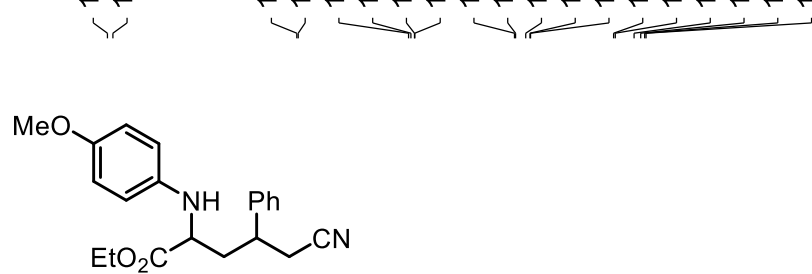

$\mathbf{3 k b}, d r=1.3: 1$

$\left(\mathrm{CDCl}_{3}, 101 \mathrm{MHz}\right)$

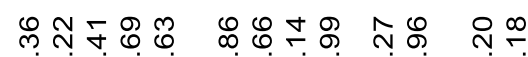

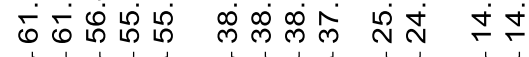

mलn

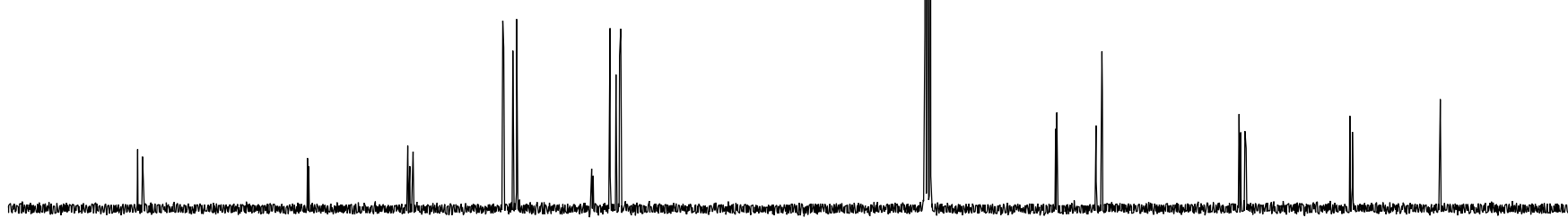




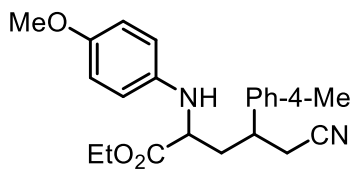

3kc, $d r=1.1: 1$

$\left(\mathrm{CDCl}_{3}, 400 \mathrm{MHz}\right)$

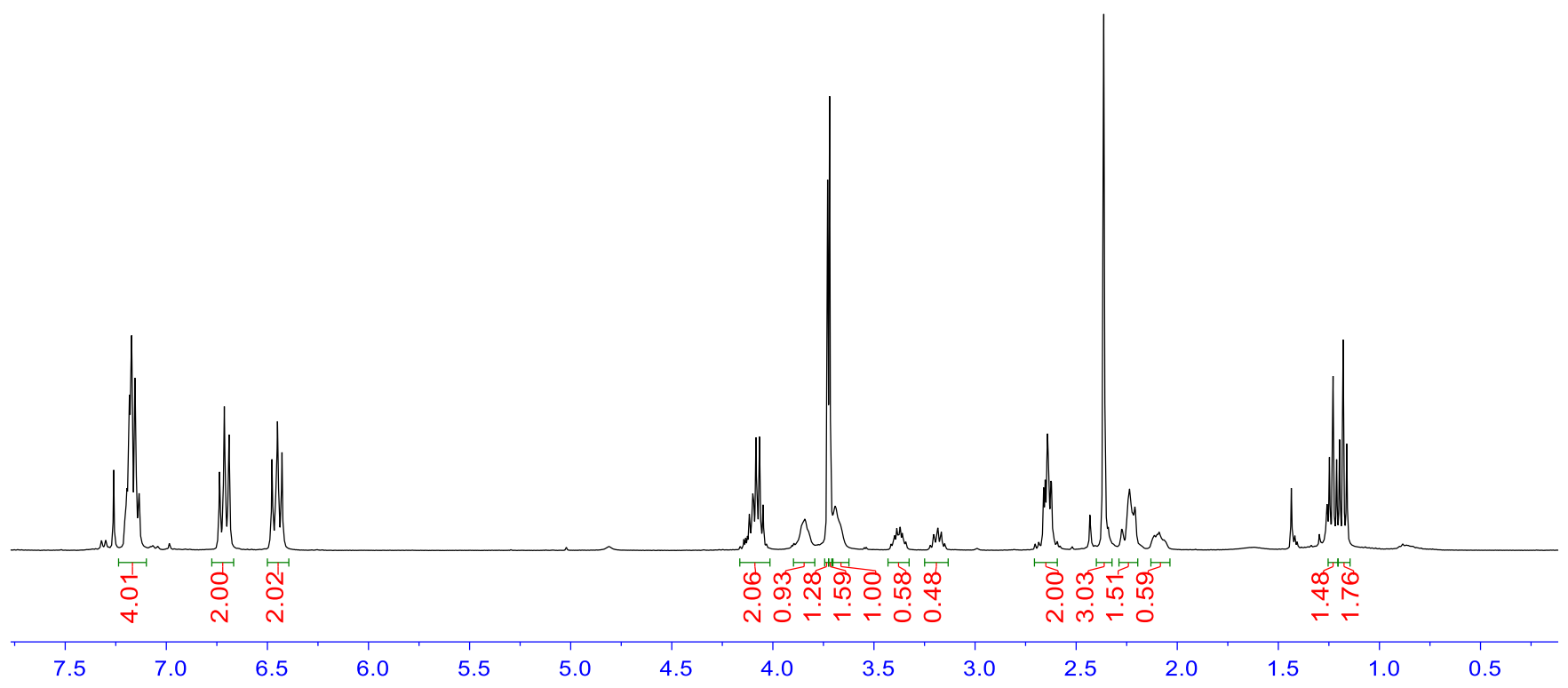

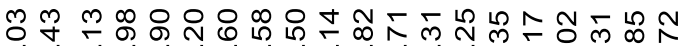

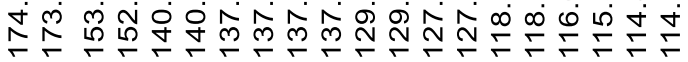

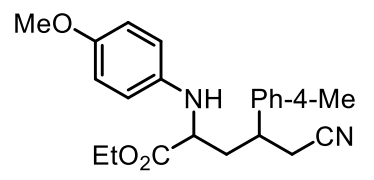

3kc, $d r=1.1: 1$

$\left(\mathrm{CDCl}_{3}, 101 \mathrm{MHz}\right)$

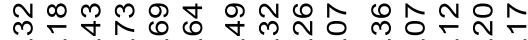

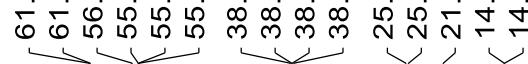

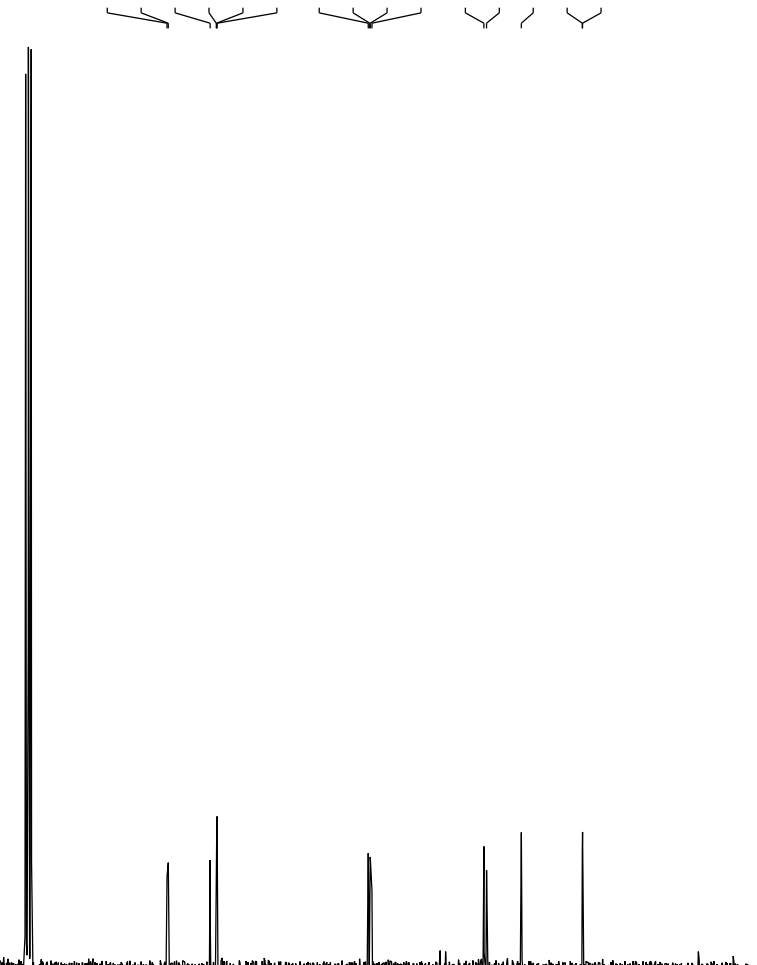


츔

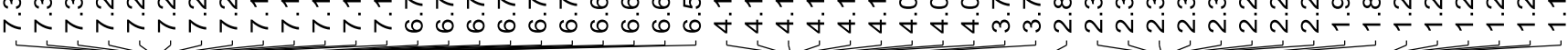

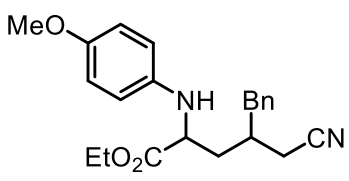

3kd, $d r=1.2: 1$

$\left(\mathrm{CDCl}_{3}, 400 \mathrm{MHz}\right)$
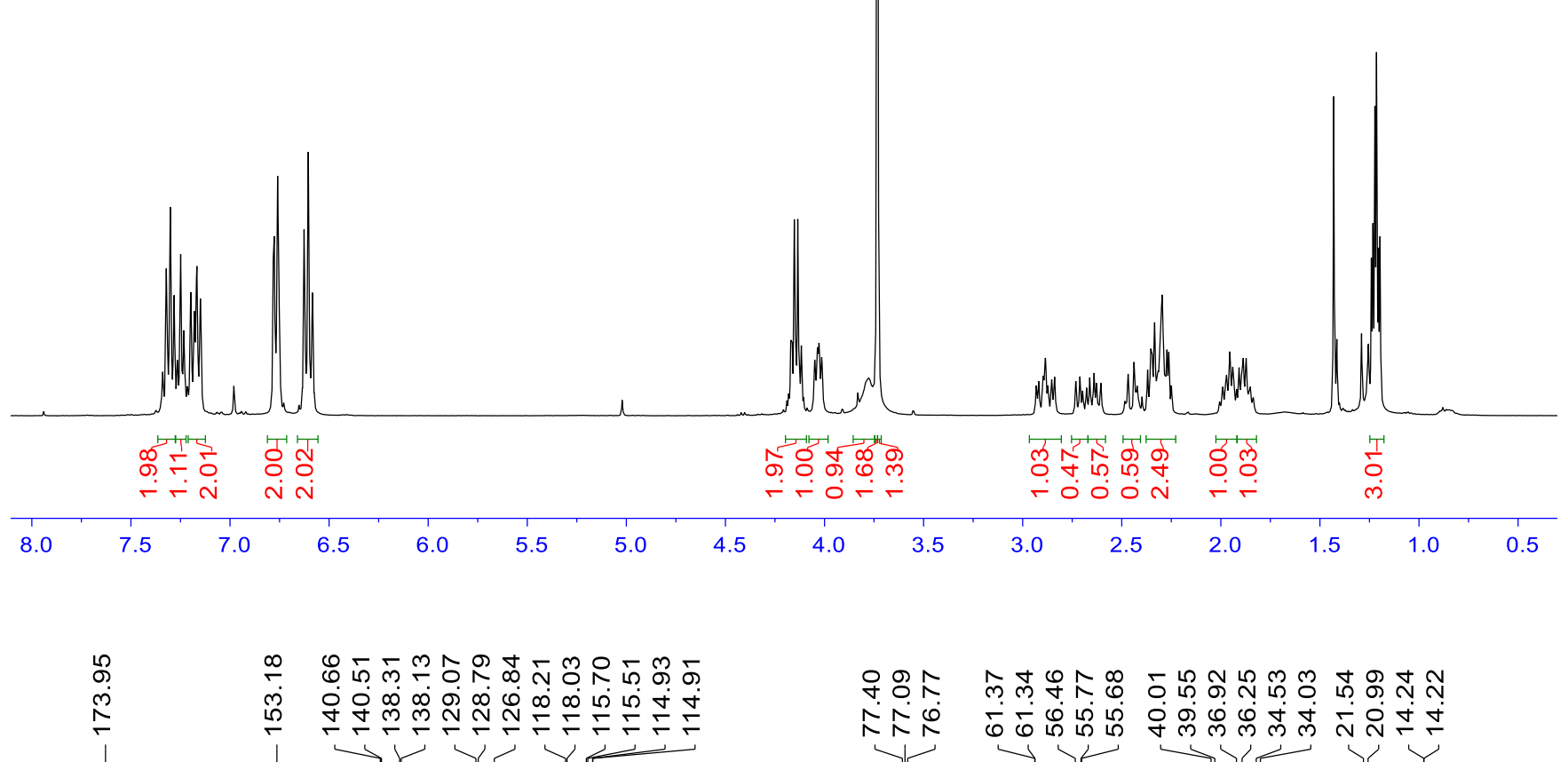

우옹

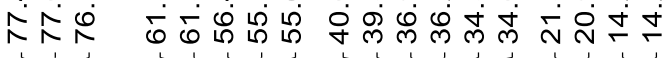
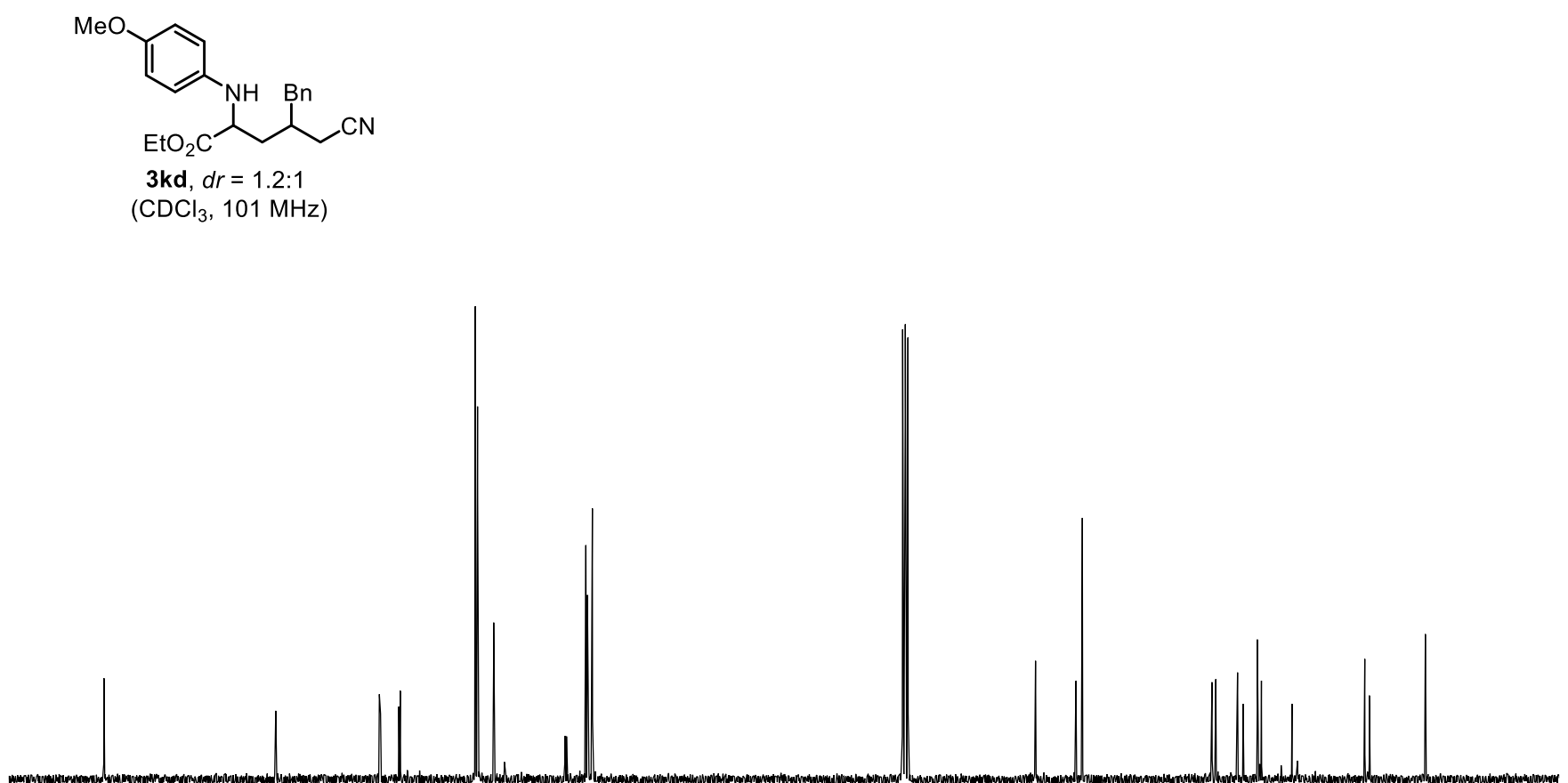


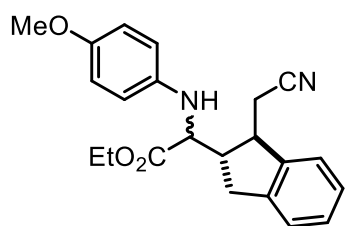

3ke, $d r=1.1:$

$\left(\mathrm{CDCl}_{3}, 400 \mathrm{MHz}\right)$

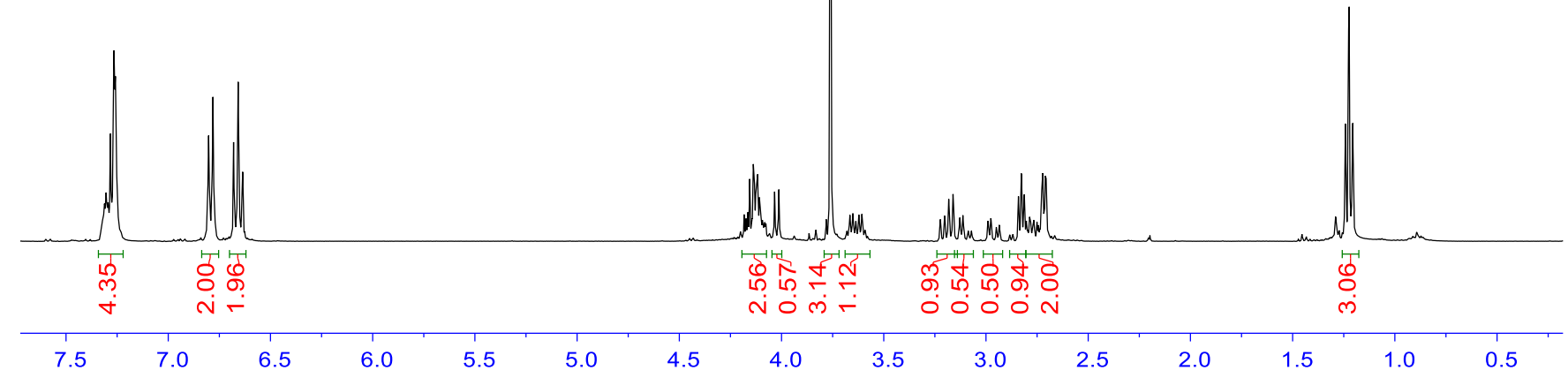

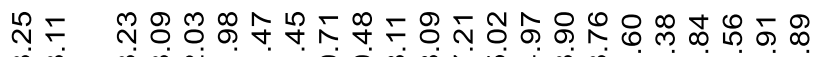

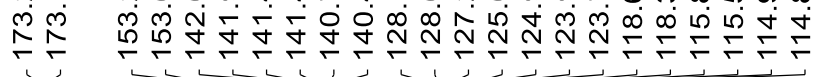

นคำ

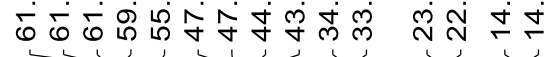<smiles>CCOC(=O)[C@H](Nc1ccc(OC)cc1)[C@H]1Cc2ccccc2C1CC#N</smiles>

3ke, $d r=1.1: 1$

$\left(\mathrm{CDCl}_{3}, 101 \mathrm{MHz}\right)$

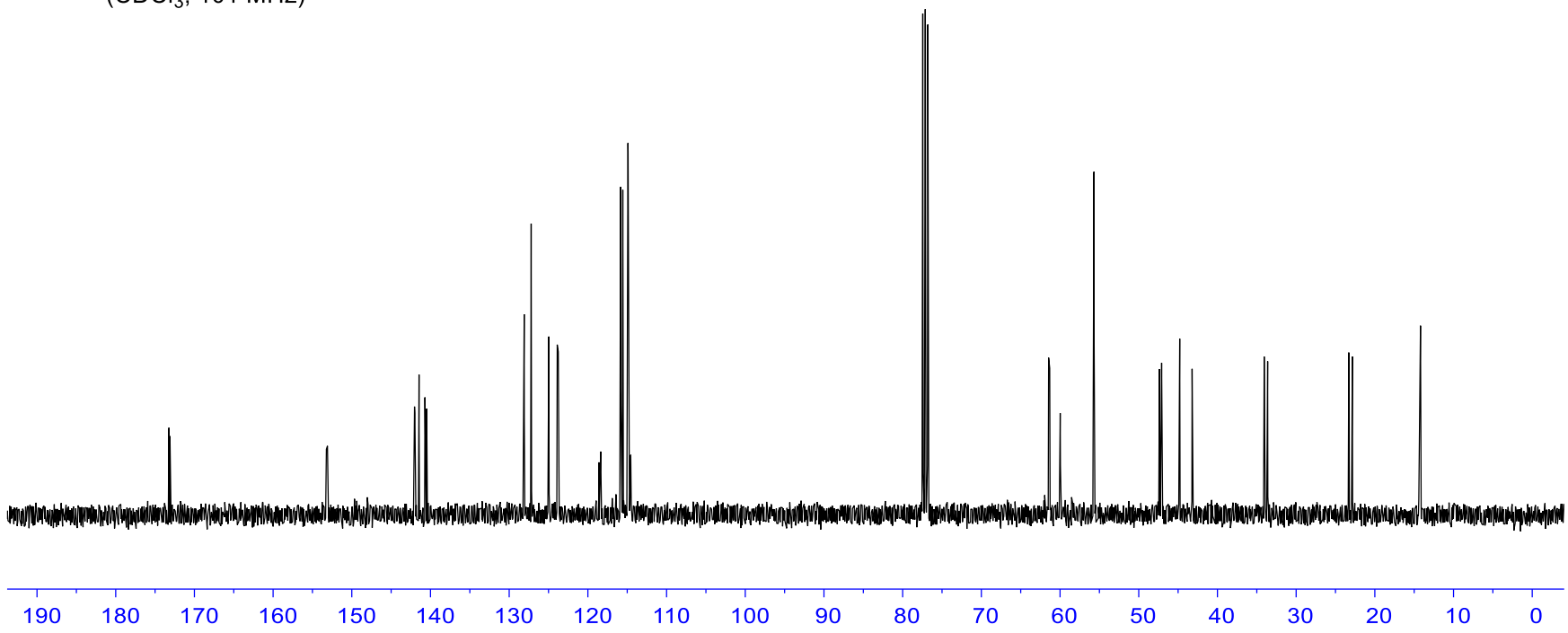


<smiles>CCOCC(Nc1ccc(OC)cc1)C(C#N)COCCO</smiles>

3kf $\left(\mathrm{CDCl}_{3}, 400 \mathrm{MHz}\right)$

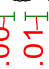

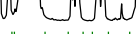

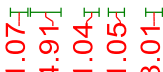
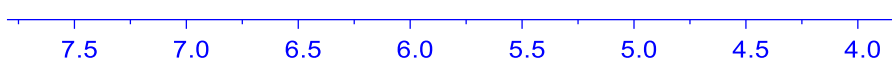

3.5

3.0

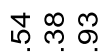

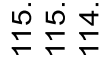

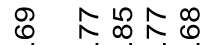

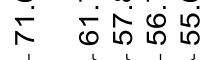

$\stackrel{+}{\frac{1}{2}}$

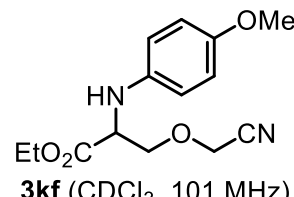

3kf $\left(\mathrm{CDCl}_{3}, 101 \mathrm{MHz}\right)$ 


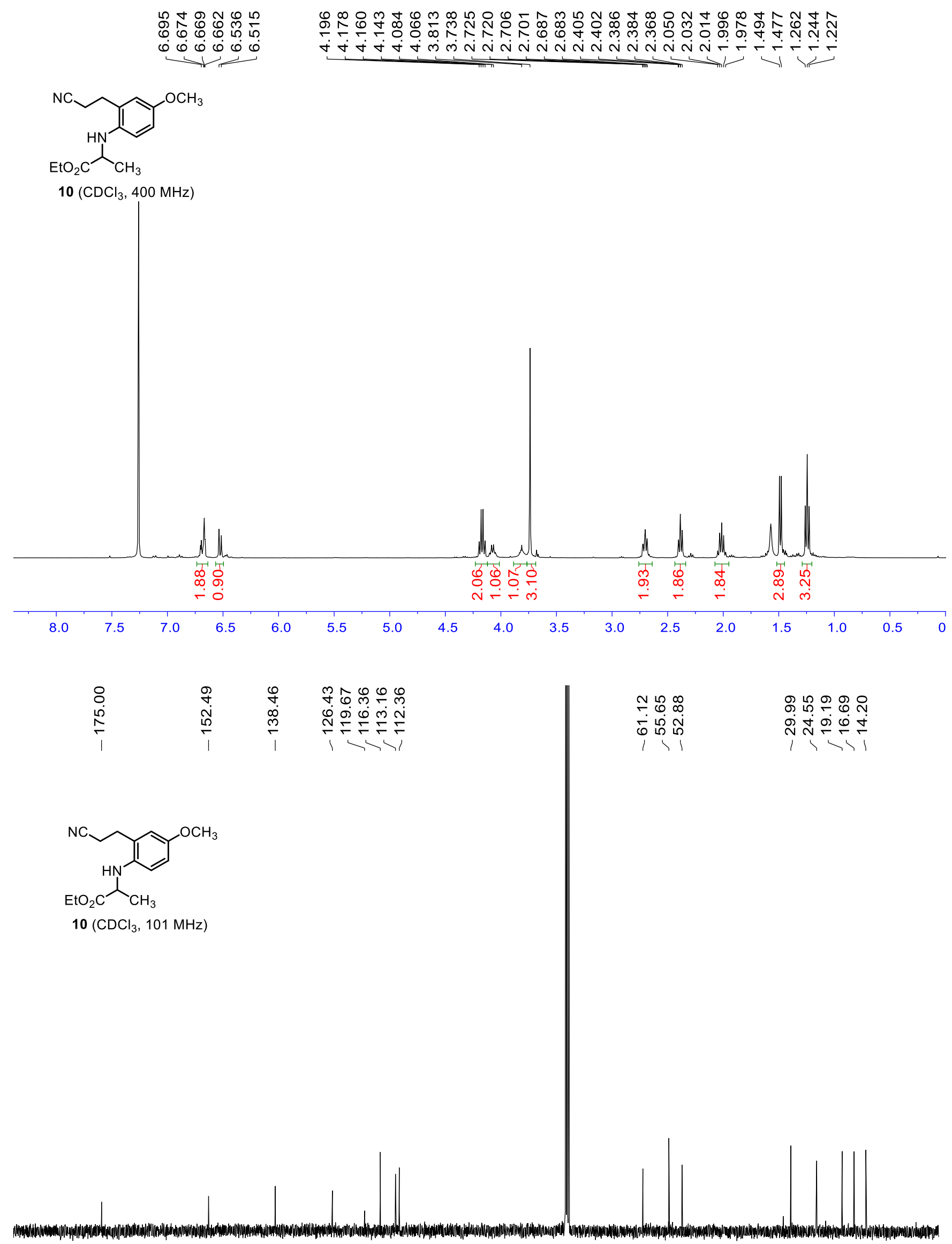




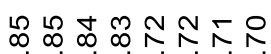

$\dot{0} \dot{0} \dot{0} \dot{0} \dot{0} \dot{0}$

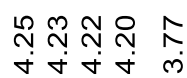

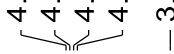

$\stackrel{\infty}{0}$<smiles>COc1ccc(NC(C)C#N)cc1</smiles>

$11\left(\mathrm{CDCl}_{3}, 400 \mathrm{MHz}\right)$

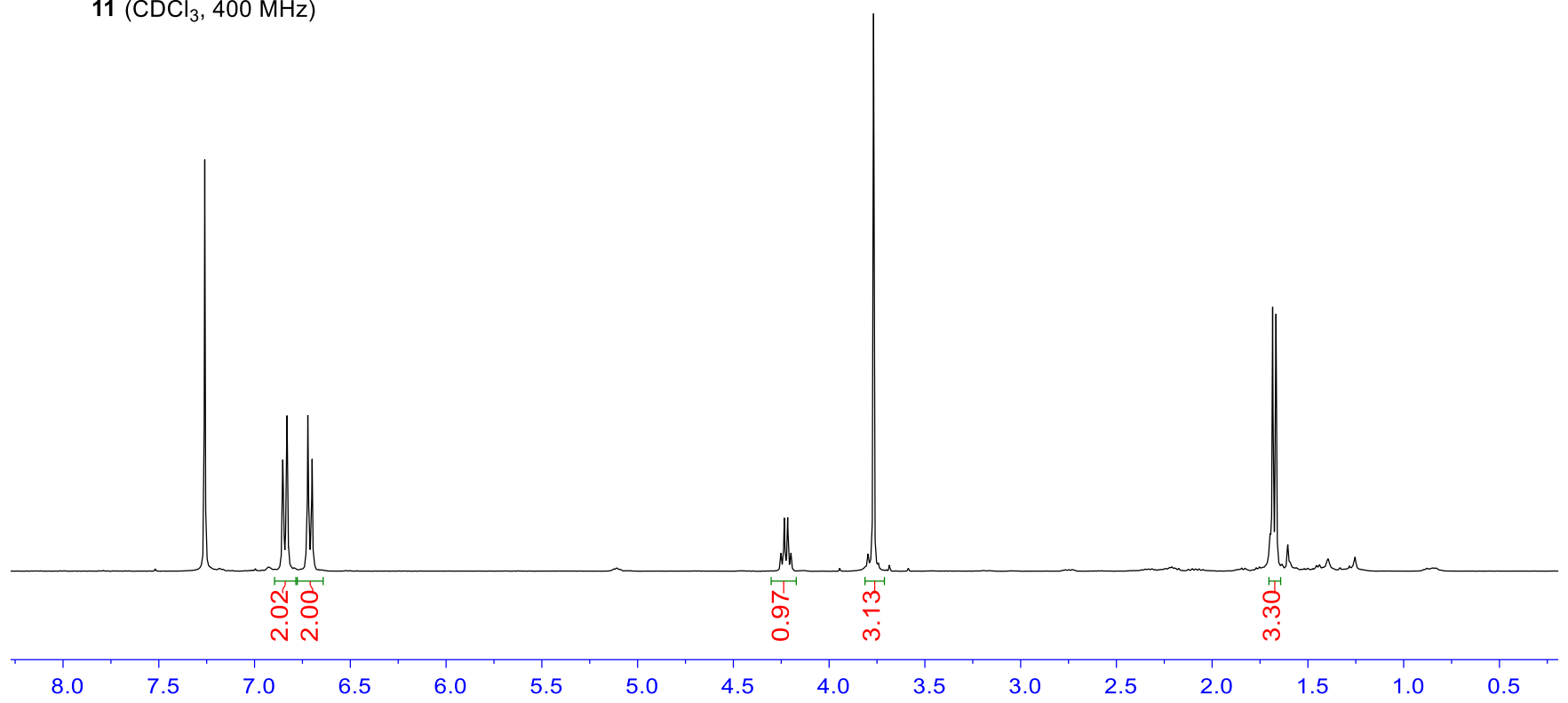

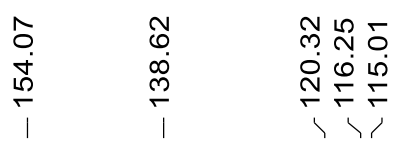

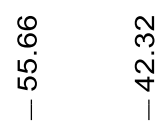

$\stackrel{5}{\infty}$
$\stackrel{1}{1}$
$\stackrel{1}{1}$

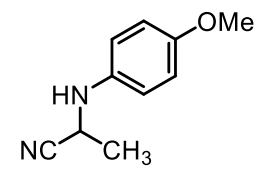

$11\left(\mathrm{CDCl}_{3}, 101 \mathrm{MHz}\right)$

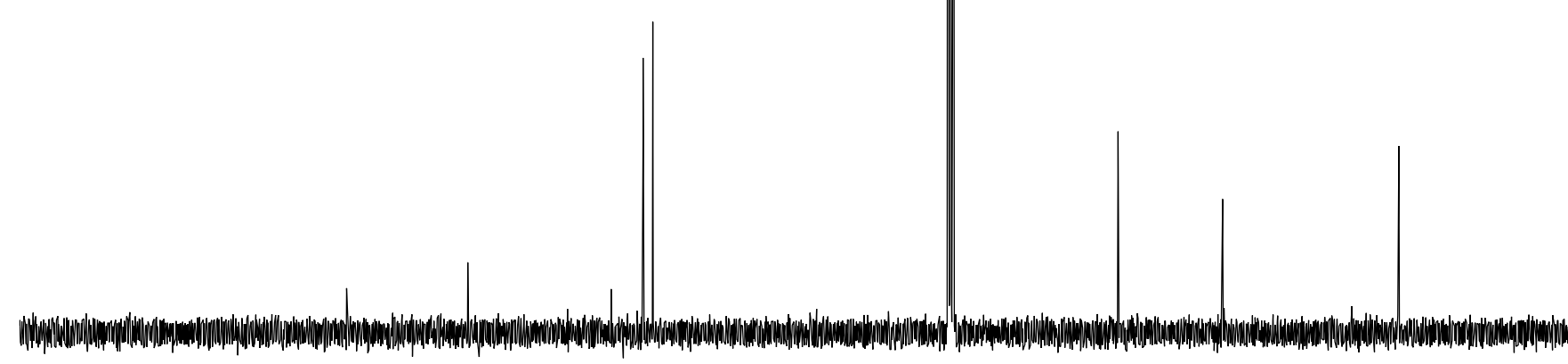




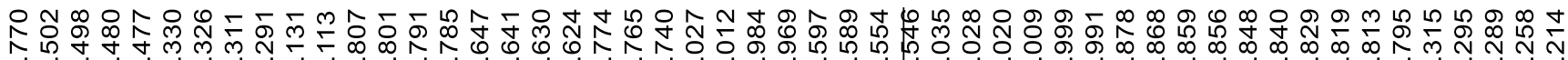
的<smiles>COc1ccc(NC(C(=O)Nc2ccccc2)C2CCCC[C@H]2CC#N)cc1</smiles>

3og, major isomer $\left(\mathrm{CDCl}_{3}, 400 \mathrm{MHz}\right)$

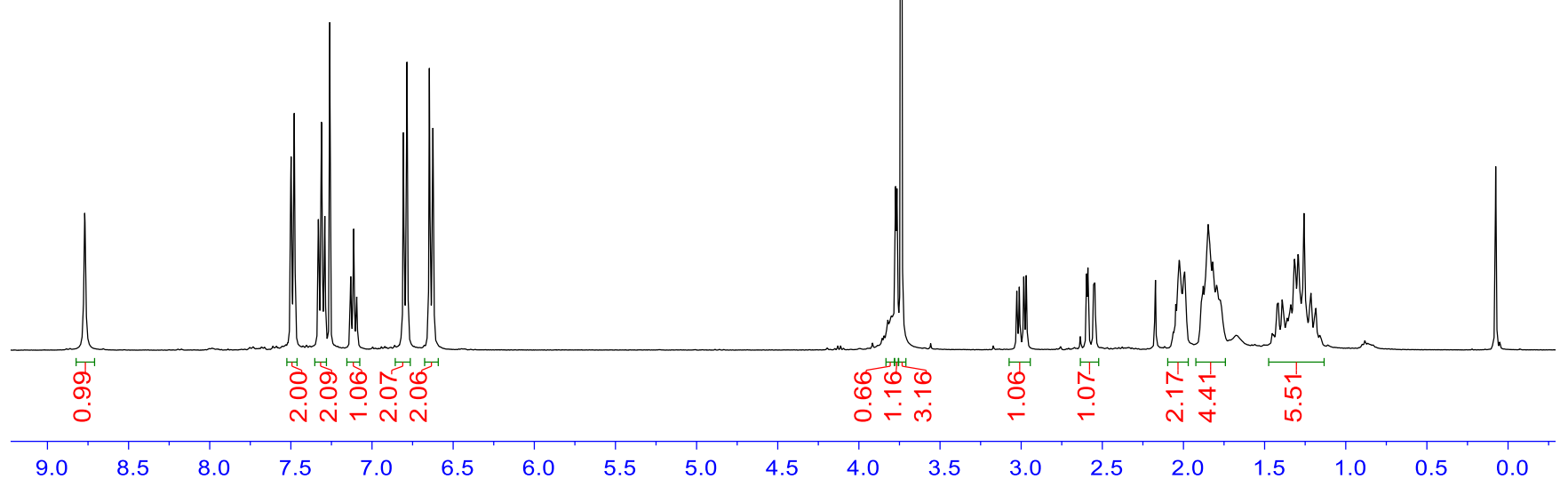

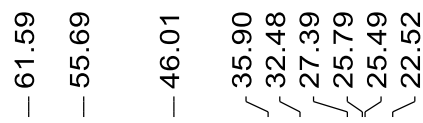

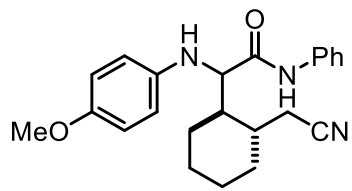

3og, major isomer

$\left(\mathrm{CDCl}_{3}, 101 \mathrm{MHz}\right)$
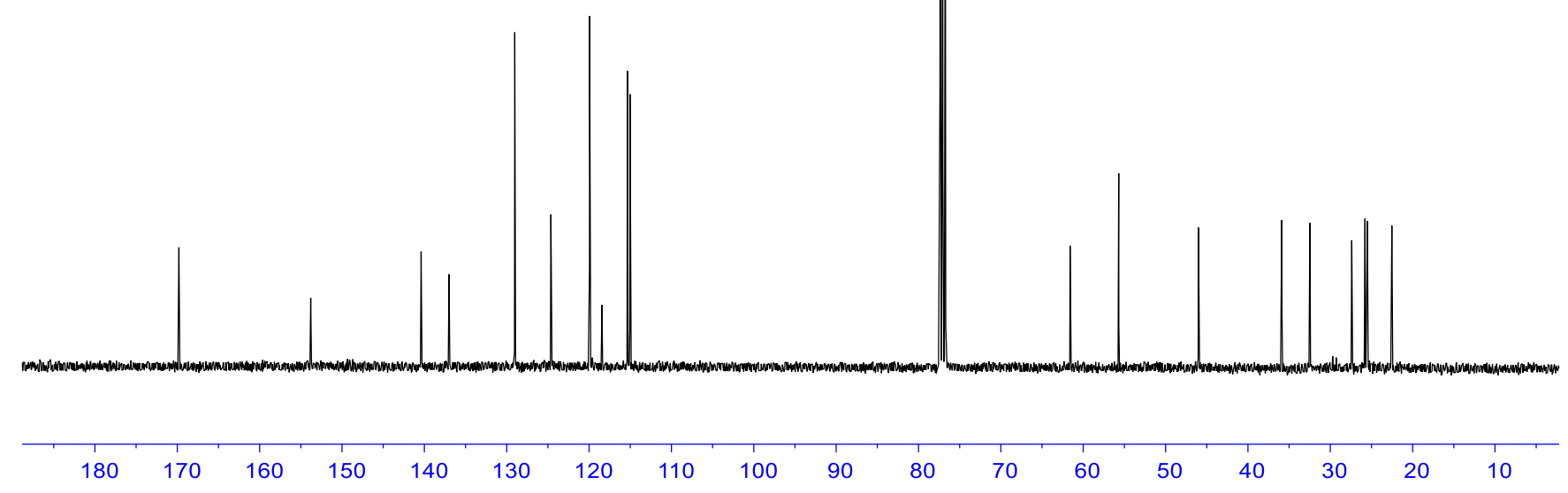


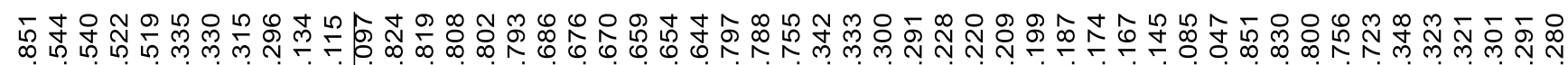
め<smiles>COc1ccc(NC(C(=O)Nc2ccccc2)C2CCCCC2C#N)cc1</smiles>

3og, minor isomer $\left(\mathrm{CDCl}_{3}, 400 \mathrm{MHz}\right)$

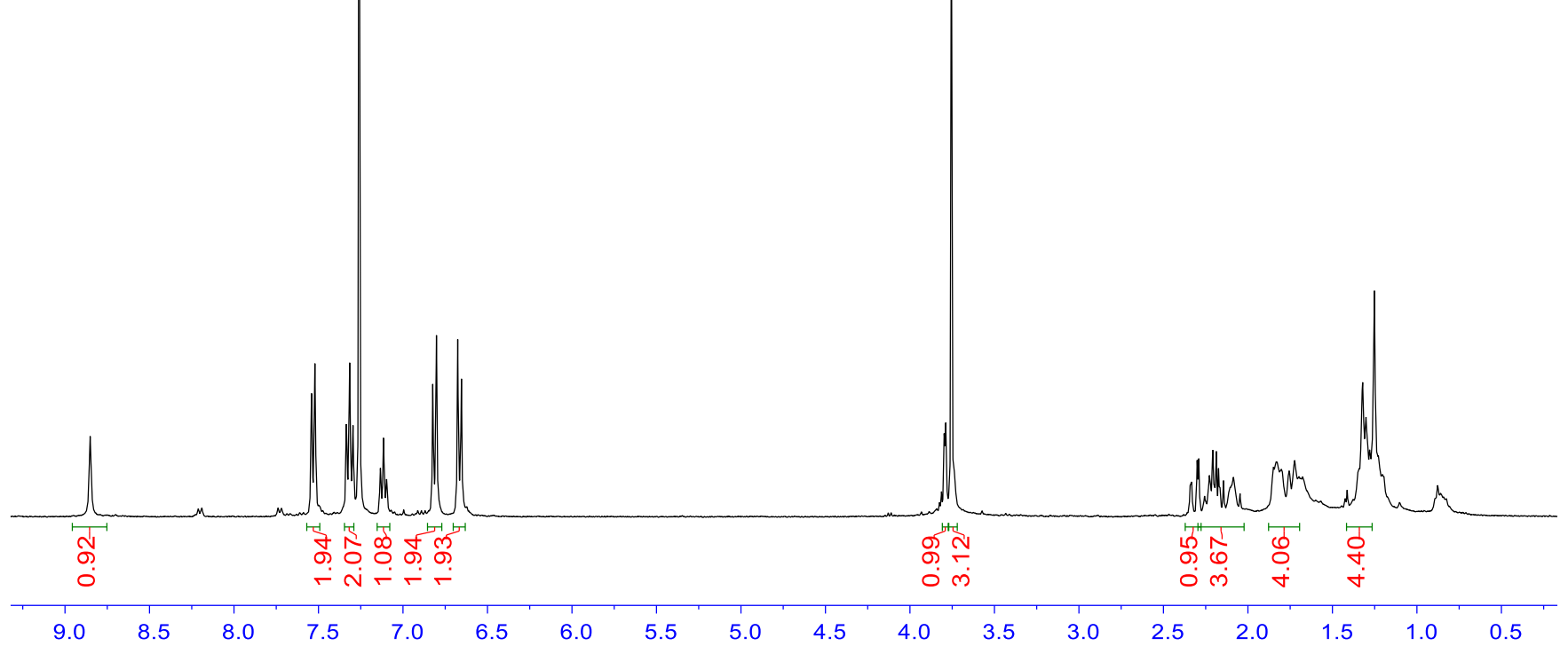

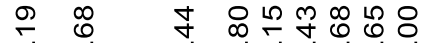

ণิ<smiles>COc1ccc(NC(C(=O)Nc2ccccc2)C2CCCCC2C#N)cc1</smiles>

3og, minor isomer $\left(\mathrm{CDCl}_{3}, 101 \mathrm{MHz}\right)$

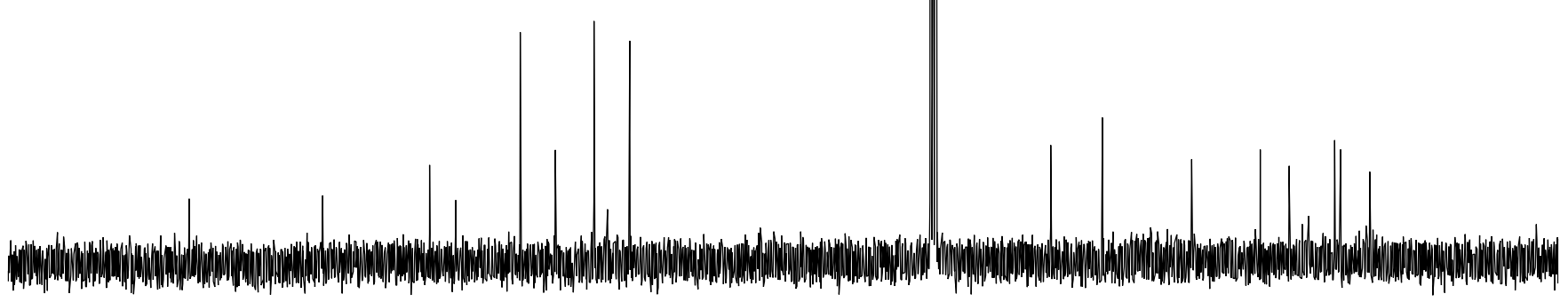


象

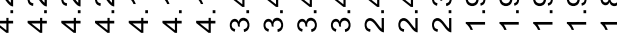<smiles>CCOC(=O)C(N)CCCC#N</smiles>

$13\left(\mathrm{CDCl}_{3}, 400 \mathrm{MHz}\right)$

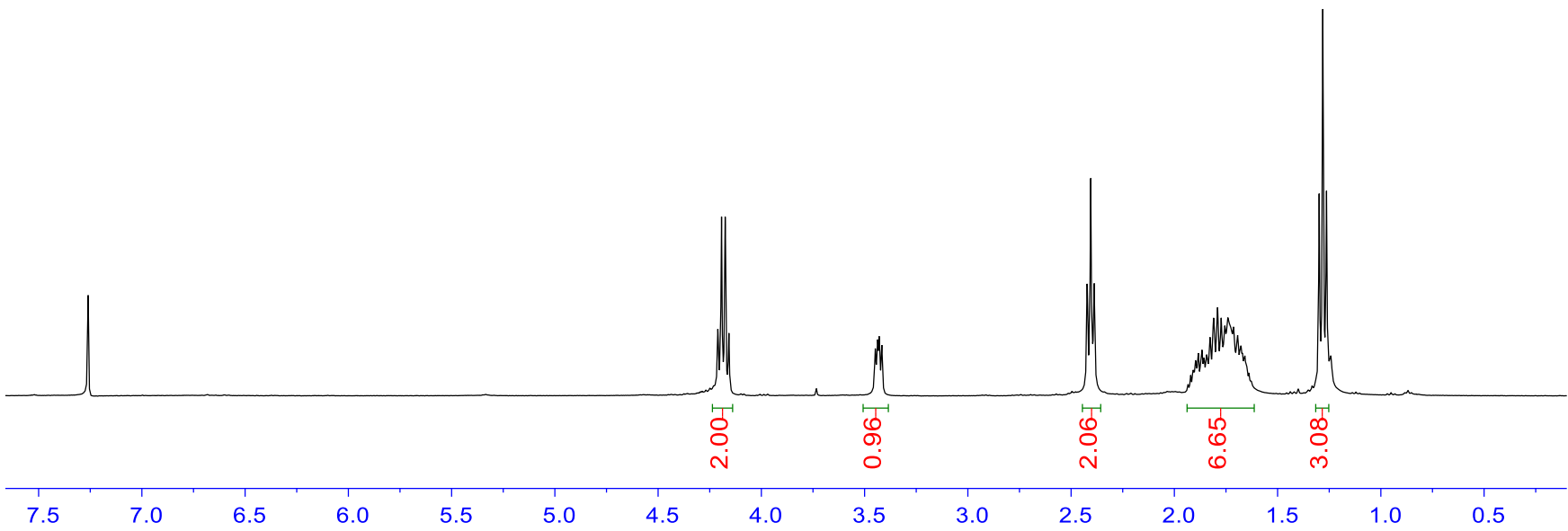

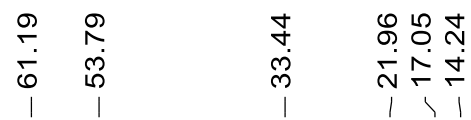<smiles>CCOC(=O)CCCCC#N</smiles>

$13\left(\mathrm{CDCl}_{3}, 101 \mathrm{MHz}\right)$

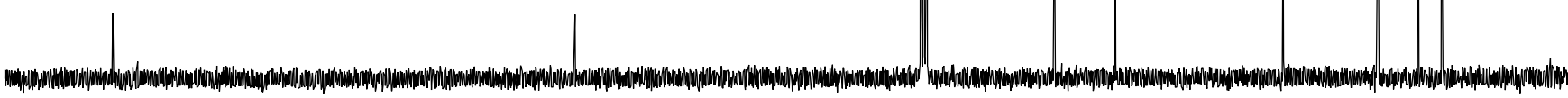




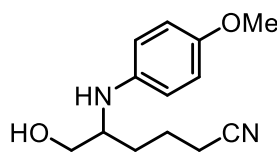

S6 $\left(\mathrm{CDCl}_{3}, 400 \mathrm{MHz}\right)$

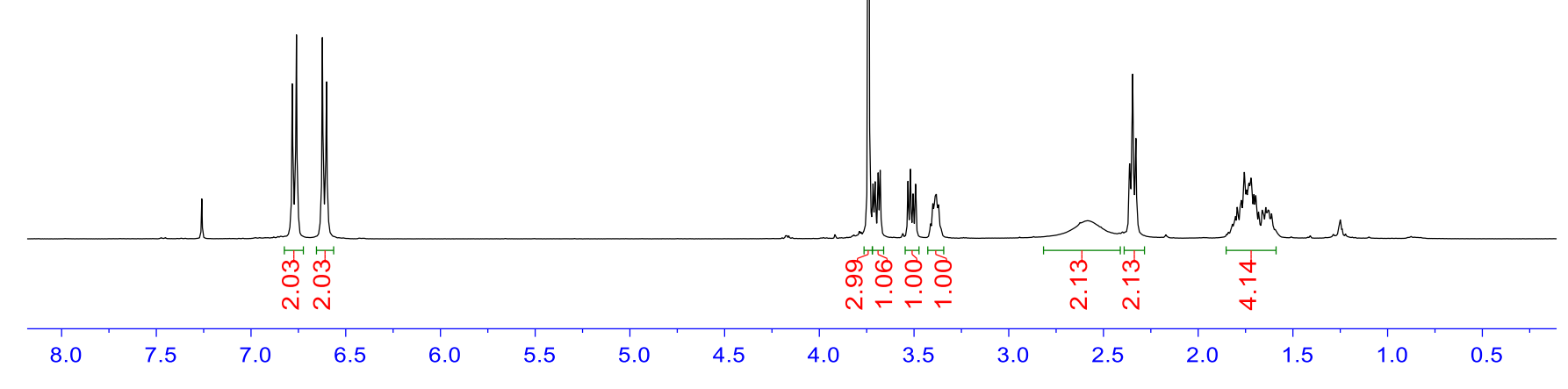

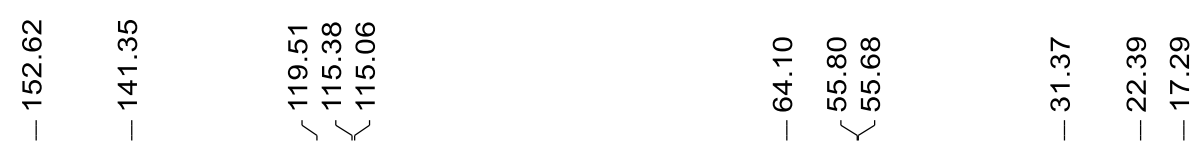
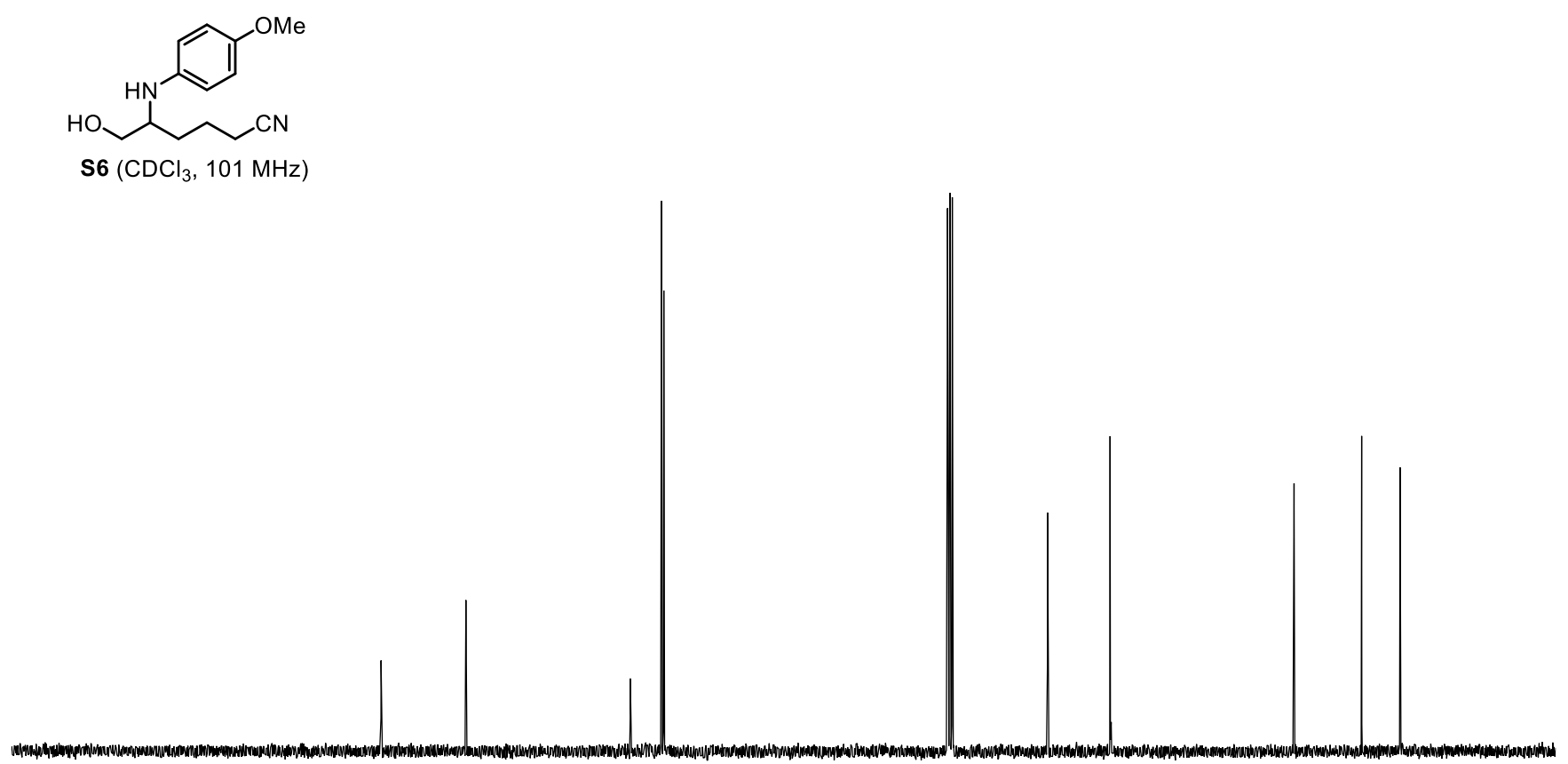

$\begin{array}{lllllllllllllllllll}200 & 190 & 180 & 170 & 160 & 150 & 140 & 130 & 120 & 110 & 100 & 90 & 80 & 70 & 60 & 50 & 40 & 30 & 20\end{array}$ 


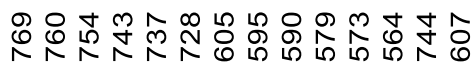

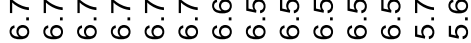

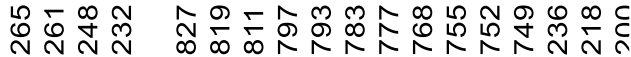
0.0000000000 in in<smiles>CCOC(=O)C(CCCC(N)=O)Nc1ccc(OC)cc1</smiles>

S7 $\left(\mathrm{CDCl}_{3}, 400 \mathrm{MHz}\right)$

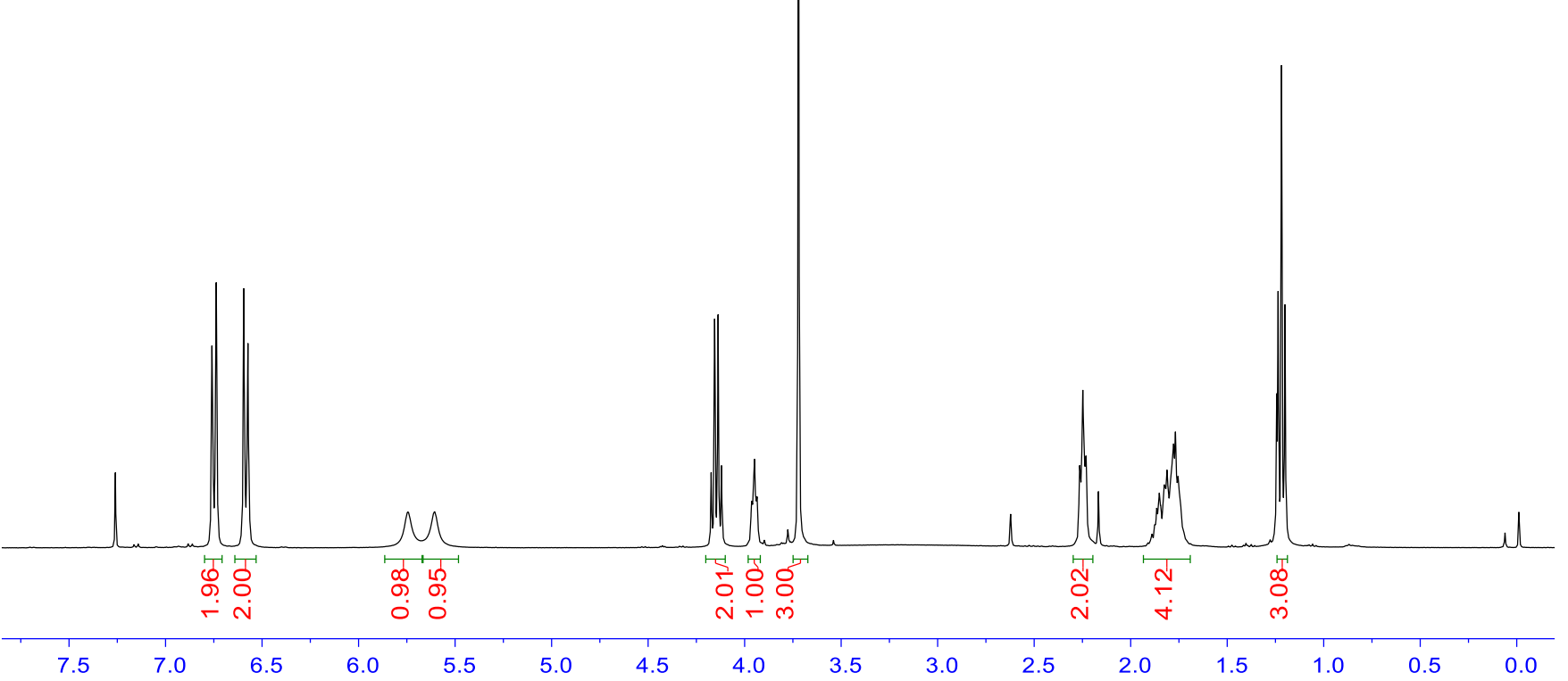

$\stackrel{\infty}{\infty} \stackrel{\infty}{\sim}$

站先

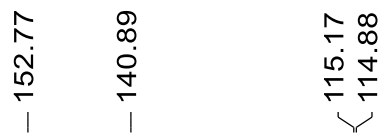

ำก

ठำ

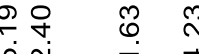

r

$\mathrm{MeO}$

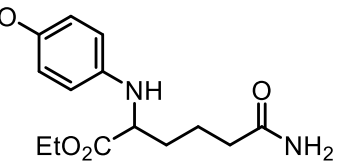

S7 $\left(\mathrm{CDCl}_{3}, 101 \mathrm{MHz}\right)$

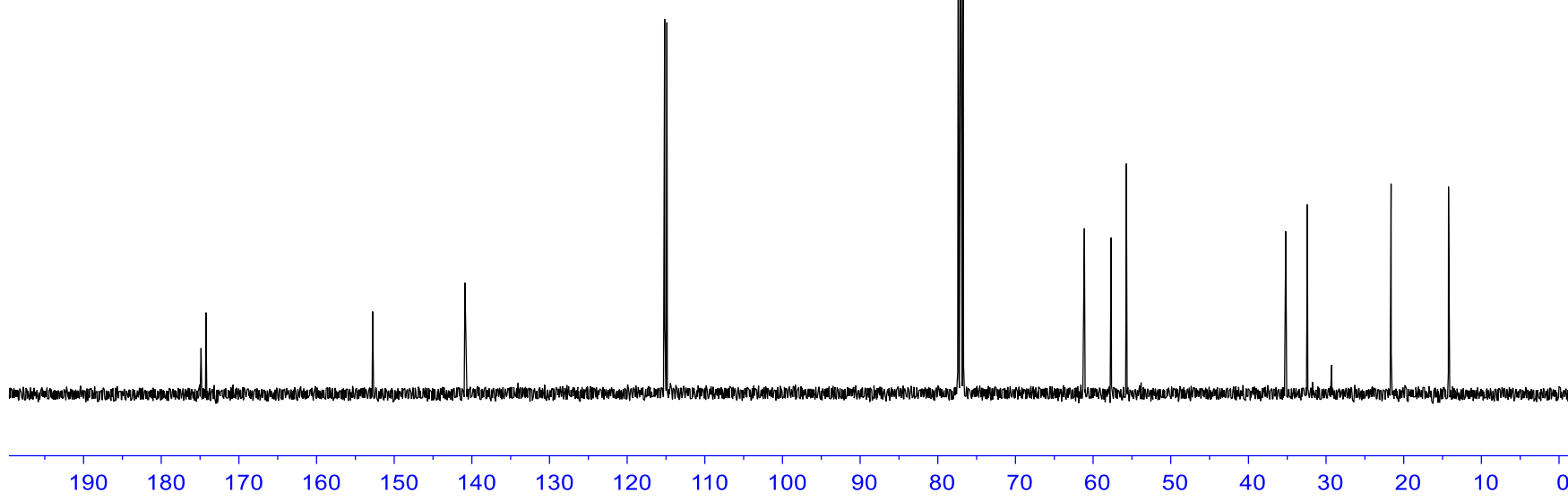




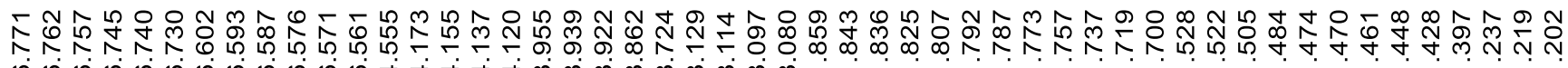

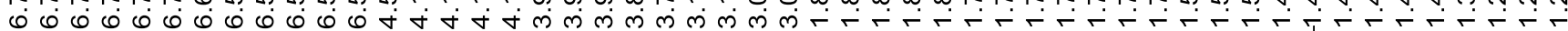<smiles>CCOC(=O)c1ccccc1NCCCCNC(=O)c1ccccc1</smiles>

S8 $\left(\mathrm{CDCl}_{3}, 400 \mathrm{MHz}\right)$

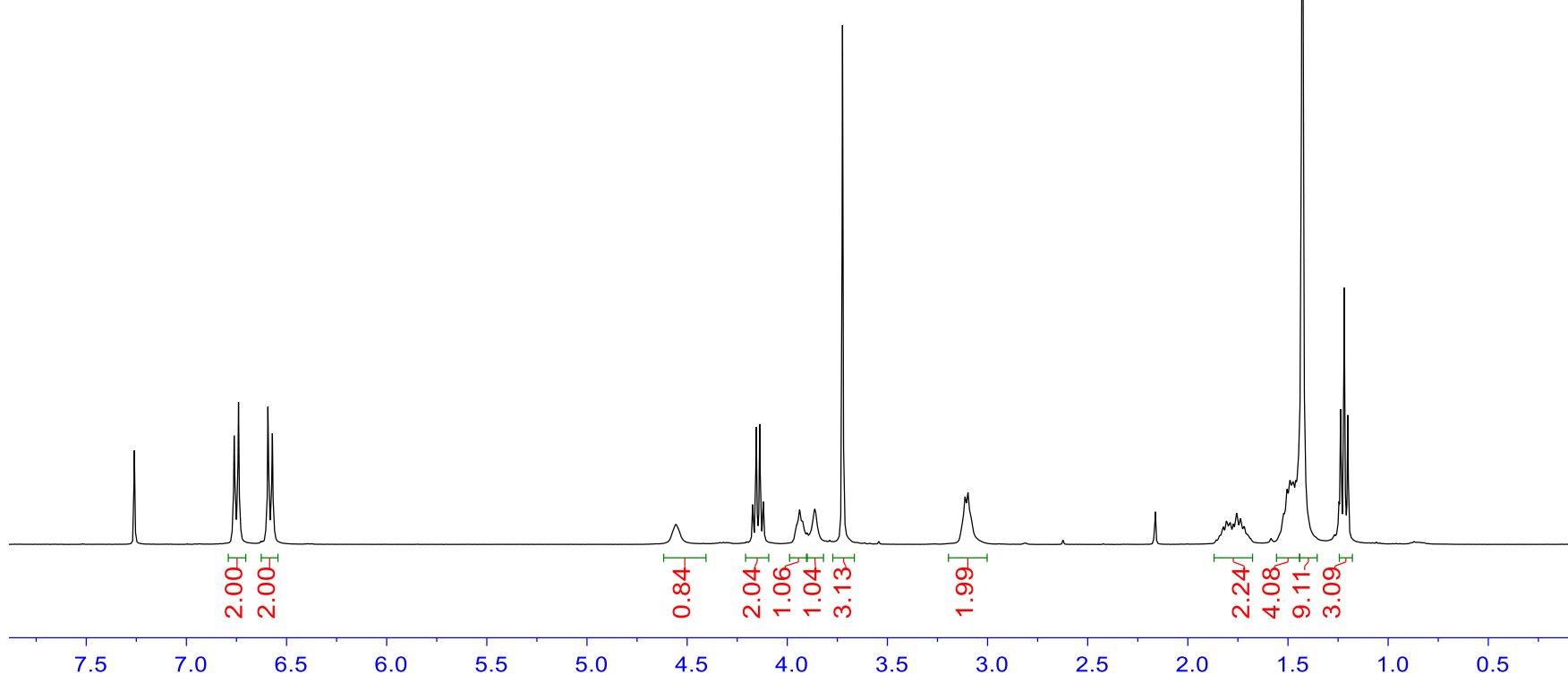

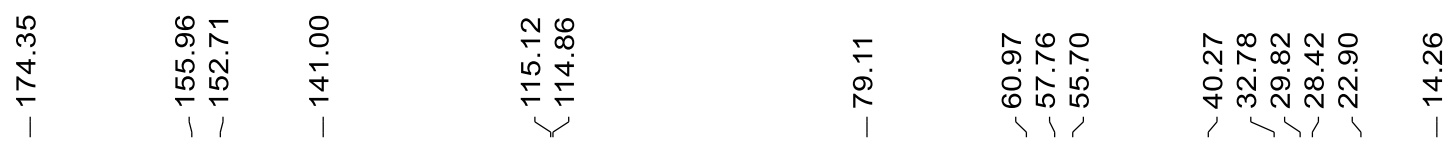

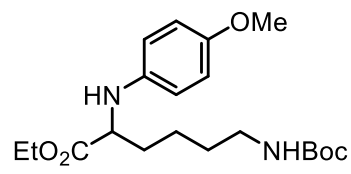

$\mathbf{s} 8\left(\mathrm{CDCl}_{3}, 101 \mathrm{MHz}\right)$

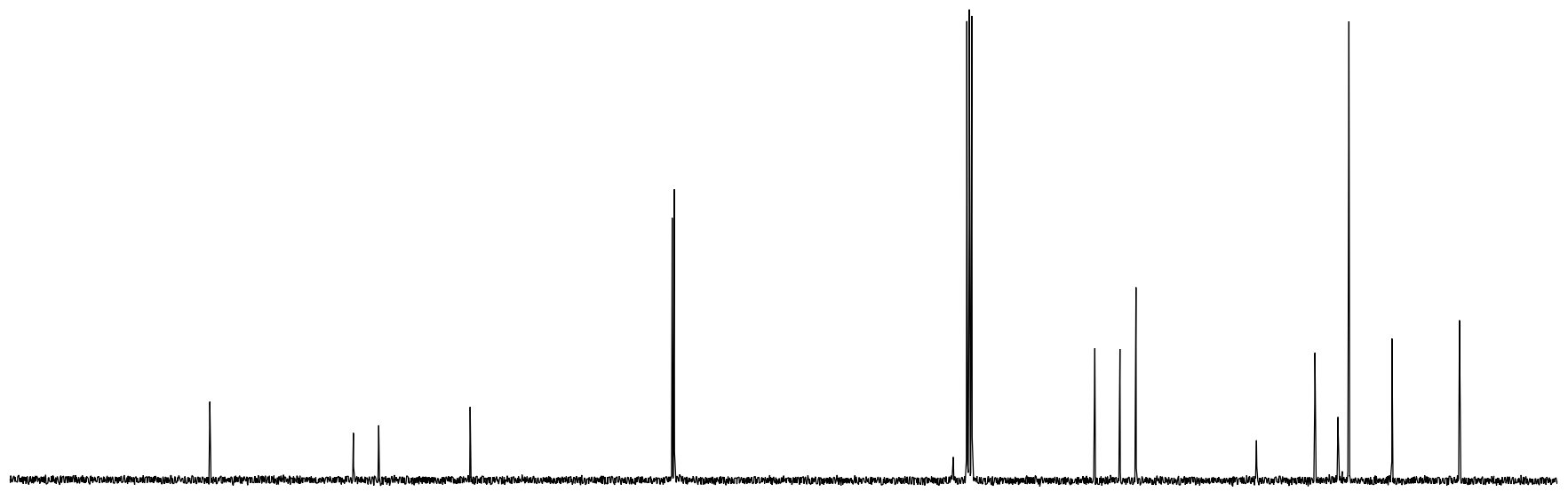

